\title{
Modeling Takotsubo syndrome with patient-specific induced pluripotent stem cell-derived cardiomyocytes
}

\author{
Dissertation \\ For the award of the degree \\ "Doctor rerum naturalium" (Dr. rer. nat.) \\ of the Georg-August-Universität Göttingen
}

Within the doctoral program

Molecular Medicine

of the Göttingen Graduate Center for Neurosciences, Biophysics and Molecular Biosciences (GGNB)

\author{
Submitted by \\ Thomas Borchert \\ (Born in Magdeburg, Germany) \\ Göttingen, November 2020
}




\section{Thesis Committee}

PD Dr. rer. nat. Katrin Streckfuß-Bömeke (Reviewer), Clinic for Cardiology and Pneumology, University Medical Center Göttingen

Prof. Dr. mult. Thomas Meyer (Second reviewer), Molecular Psychocardiology, University Medical Center Göttingen

Prof. Dr. med. Michael Zeisberg, Nephrology and Rheumatology, University Medical Center Göttingen

\section{Members of the Examination Board}

$1^{\text {st }}$ Referee: $\quad$ Prof. Dr. med. Ralf Dressel, institute for cellular and molecular immunology, Göttingen.

$2^{\text {nd }}$ Referee: $\quad$ Prof. Dr. rer. nat. Rüdiger Behr, German Primate Center, Göttingen.

$3^{\text {rd }}$ Referee: $\quad$ PD Dr. rer. nat. Laura Zelarayán-Behrend, Institute for Pharmacology and Toxicology, University Medical Center Göttingen

Date of oral examination: $11^{\text {th }}$ December 2020 


\section{Table of contents}

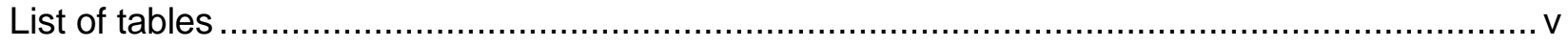

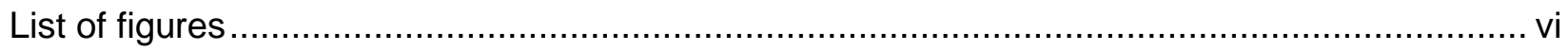

Summary

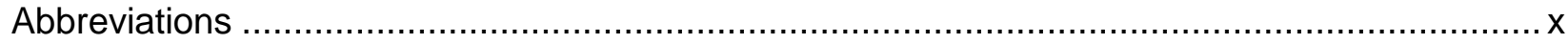

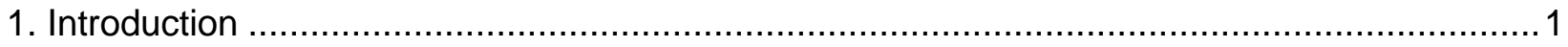

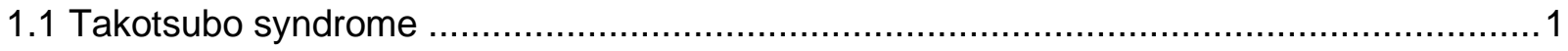

1.1.1 Clinical presentation of Takotsubo syndrome ……................................................ 1

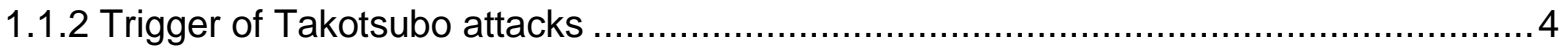

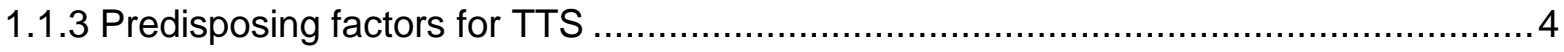

1.1.4 A variety of hypotheses try to explain the TTS phenotype ....................................

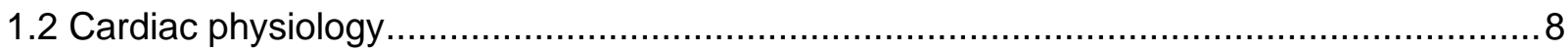

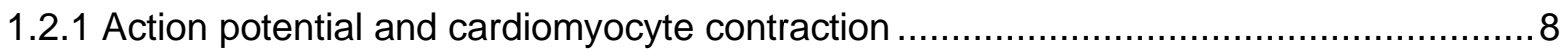

1.2.2 The $\beta$-adrenergic system in cardiomyocytes regulates beating activity ...................10

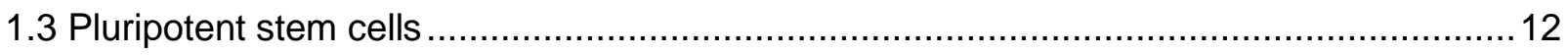

1.3.1 Multipotent stem cells are found in the human body ........................................12

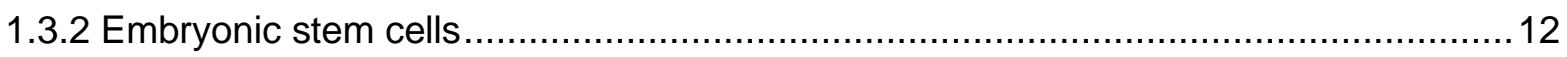

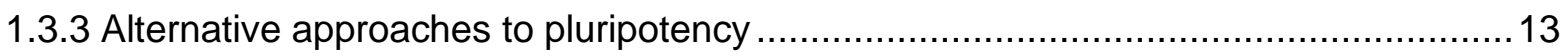

1.3.4 Induced pluripotent stem cells - a major breakthrough ........................................ 14

1.3.5 The Yamanaka factors trigger a complex pluripotent network activation in cells ........15

1.3.6 iPSCs can be differentiated towards a wide range of cells ...................................17

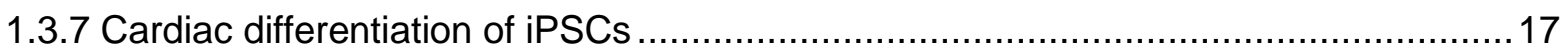

1.3.8 Disease modeling with the help of iPSCs - a step towards future drug screenings ....19

1.3.9 Role of iPSCs in the field of regenerative medicine ..........................................20

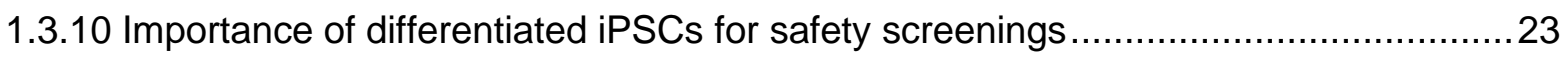

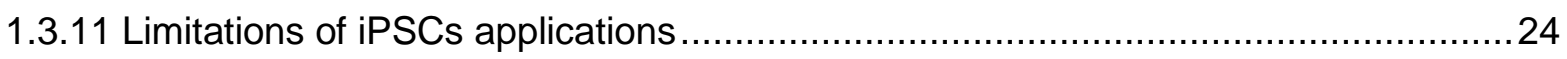

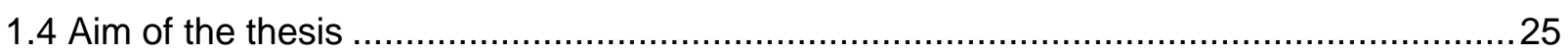

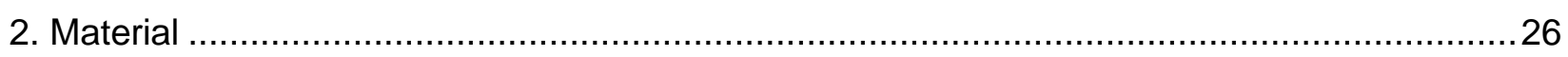

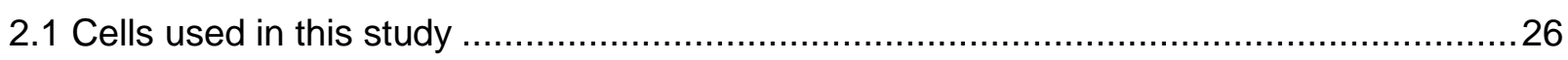

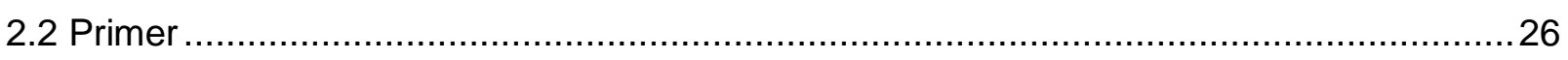

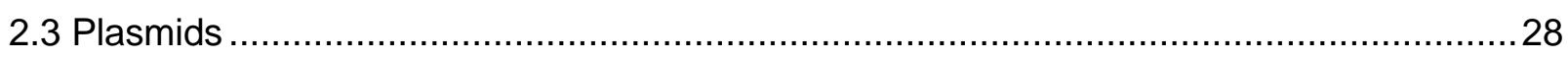

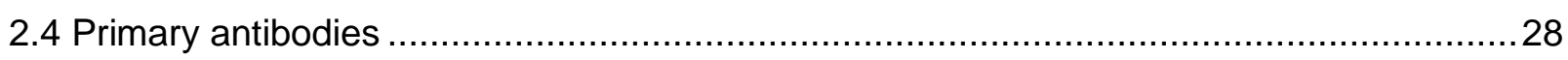

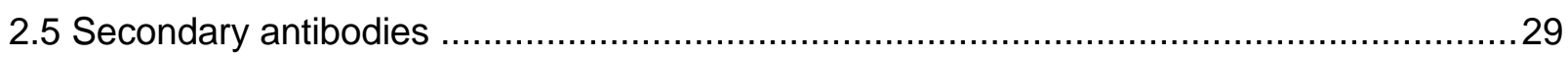

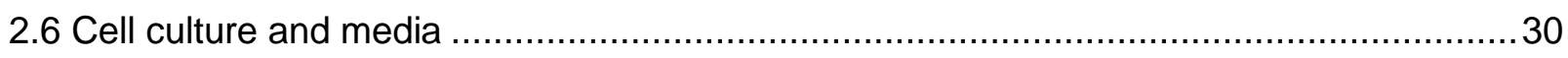




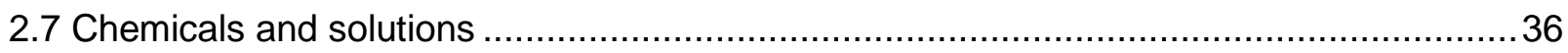

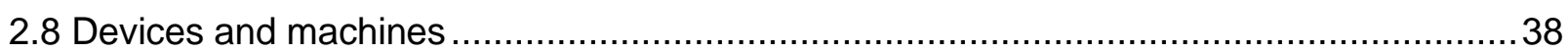

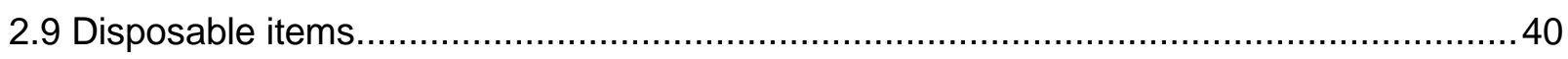

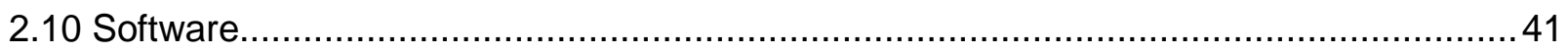

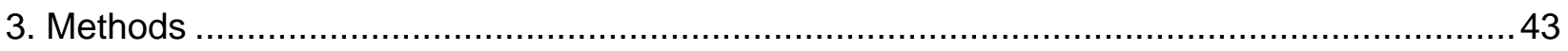

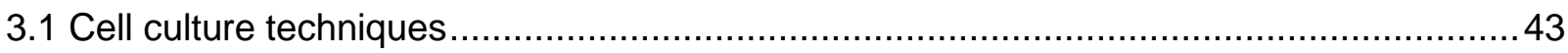

3.1.1 Fibroblast isolation from skin punch biopsies ............................................... 43

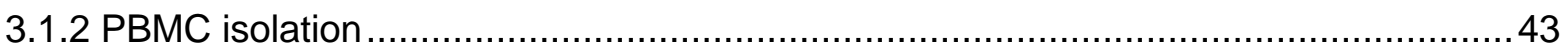

3.1.3 IPSC reprogramming of fibroblasts and PBMCS ........................................... 44

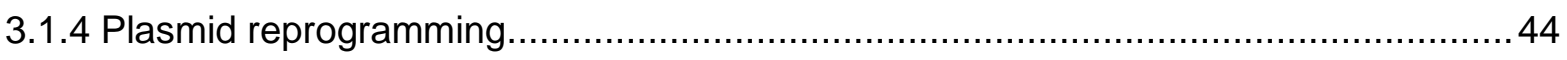

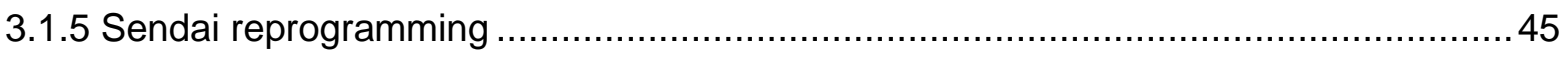

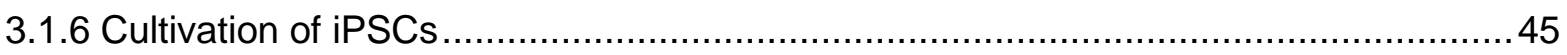

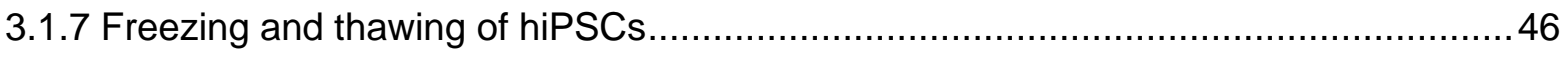

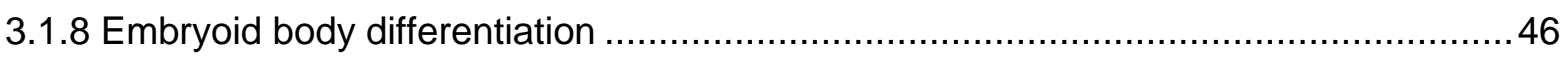

3.1.9 In vivo differentiation of iPSCs in SCID mice ............................................... 47

3.1.10 Directed in vitro iPSC-differentiation, cardiomyocyte culturing, metabolic selection

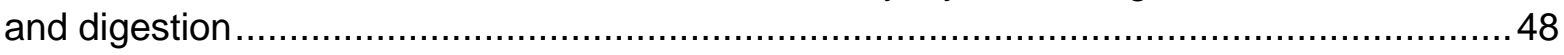

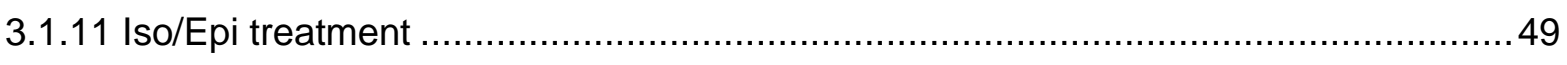

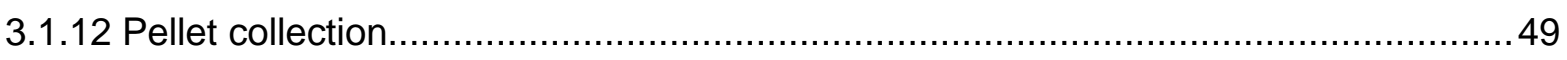

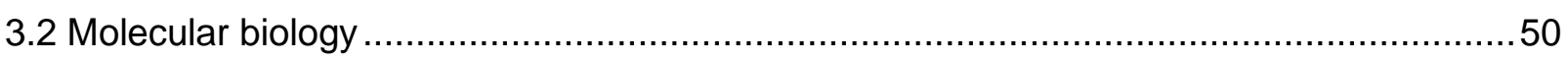

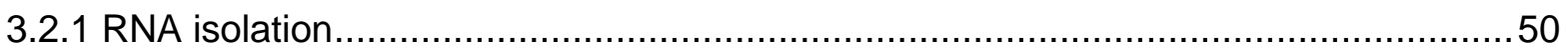

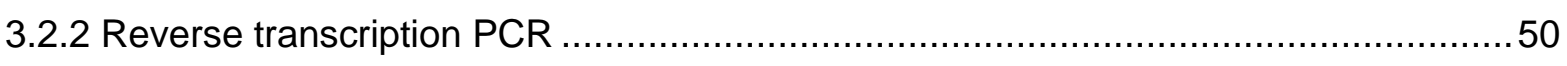

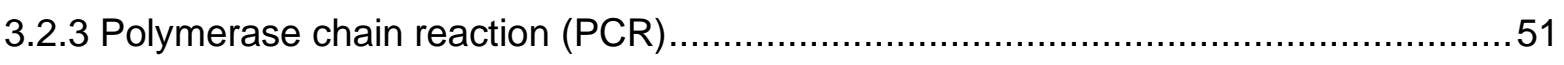

3.2.4 Quantitative PCR ....................................................................................... 52

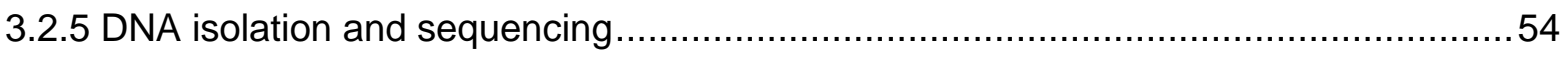

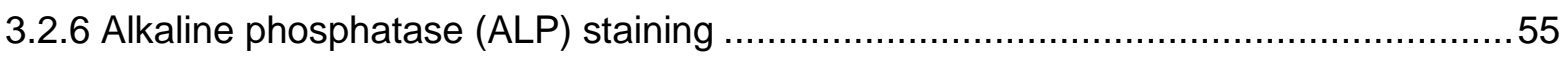

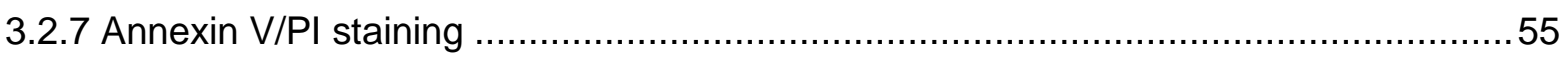

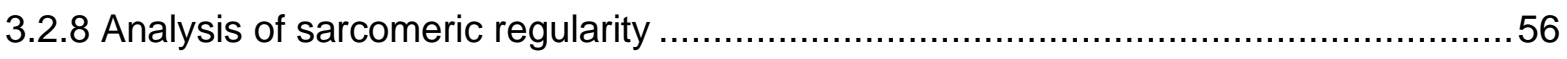

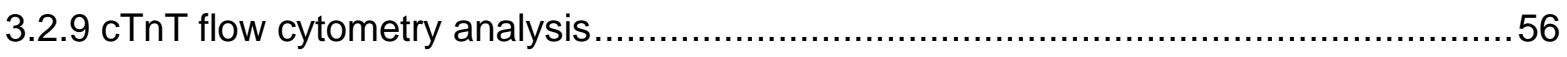

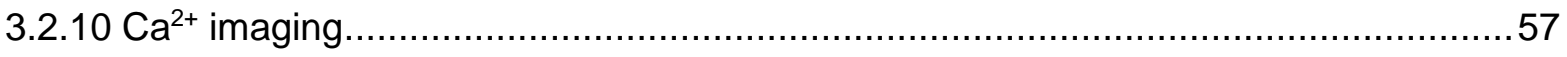

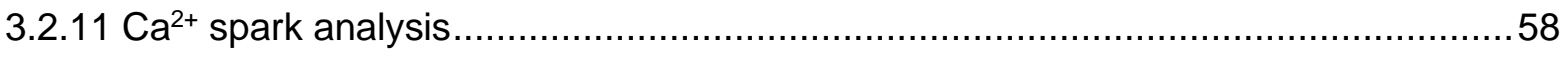

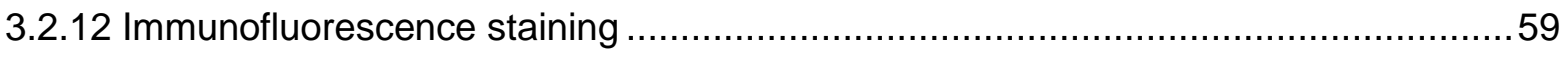

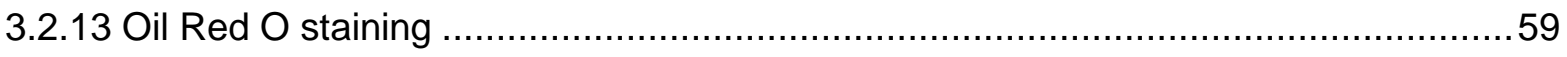

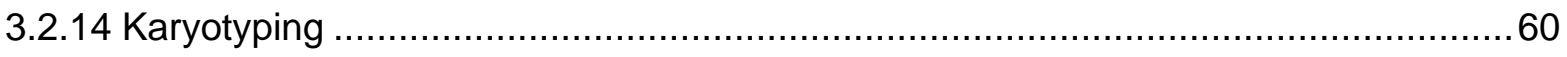

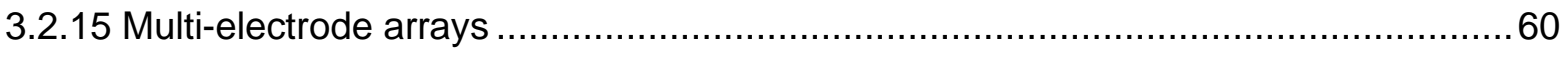


3.2.16 Statistical analysis 61

4. Results

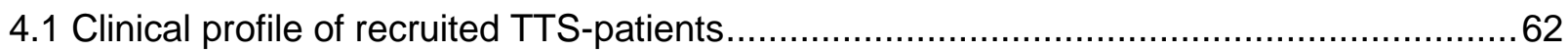

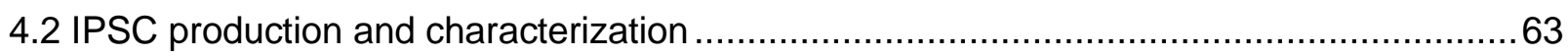

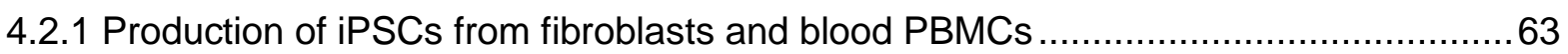

4.2.2 IPSCs show stem cell morphology and express pluripotency markers.......................64

4.2.3 iPSCs can be differentiated into cells of all three germ layers ................................66

4.2.4 IPSCs can be directly differentiated into functional cardiomyocytes..........................69

4.3 Stress can be induced by the addition of catecholamines ...........................................

4.4 Catecholamines cause electrical disturbances .......................................................

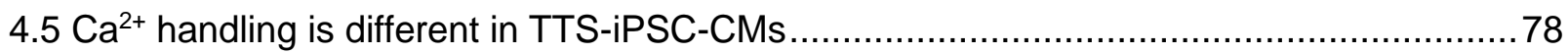

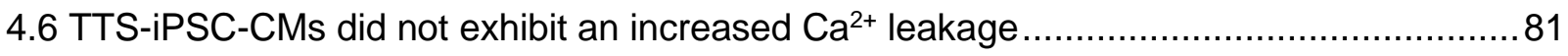

4.7 Engineered heart muscles reveal a higher sensitivity of TTS-iPSC-CMs towards Iso

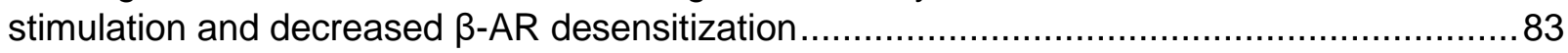

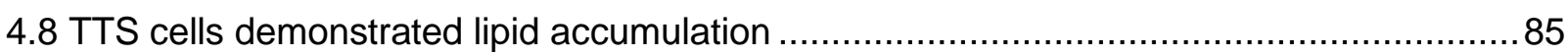

4.9 Inhibition of selected $\beta$-pathways uncovers the role of different $\beta$-AR sub-receptors .........87

4.9.1 MEA measurements emphasize the roles of different $\beta$-ARs in TTS-iPSC-CMs ........87

4.9.2 $\mathrm{Ca}^{2+}$ measurements support the dominant role of $\beta_{2}$-ARs in CTRL-iPSC-CMs and $\beta_{1}$ -

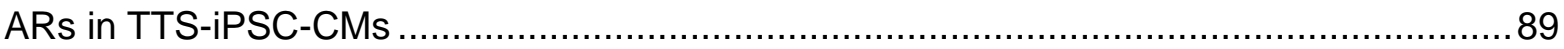

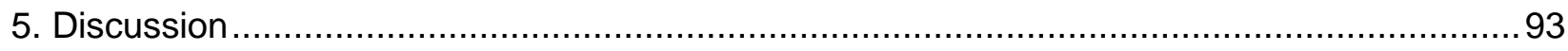

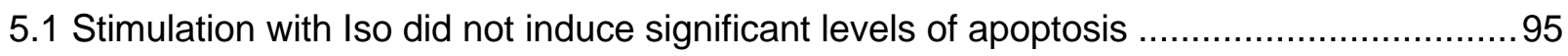

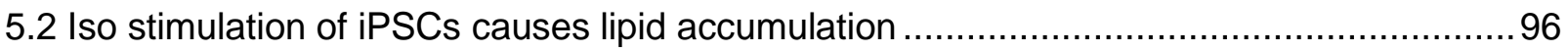

5.3 Electrical activity analysis shows that TTS-iPSC-CMs react stronger towards Iso than

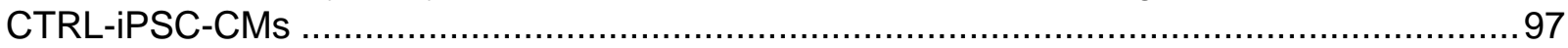

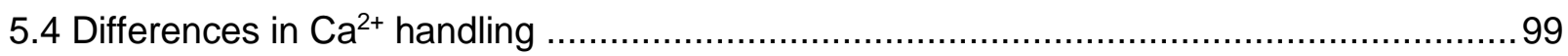

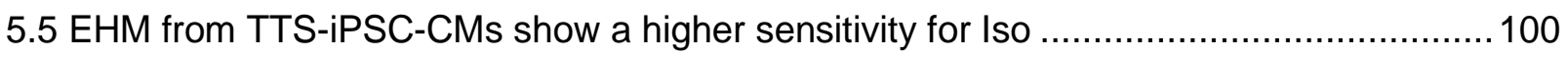

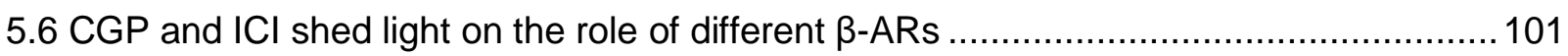

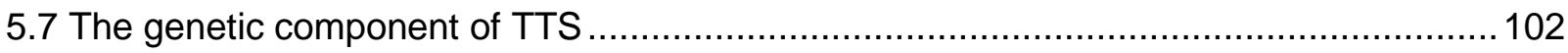

5.8 TTS triggering catecholamine levels differ between models and patients..................... 104

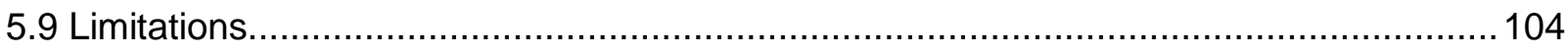

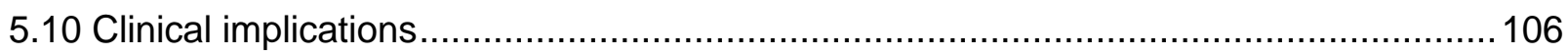

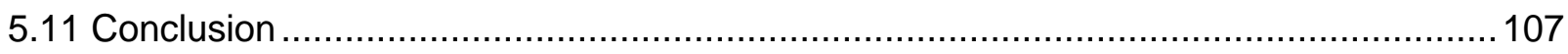

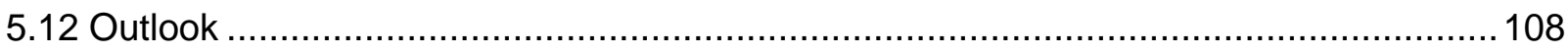

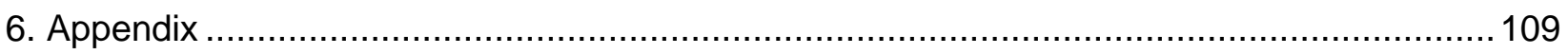

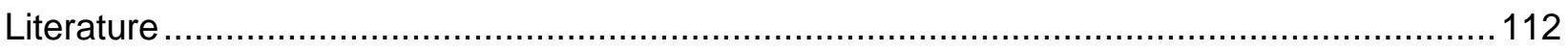




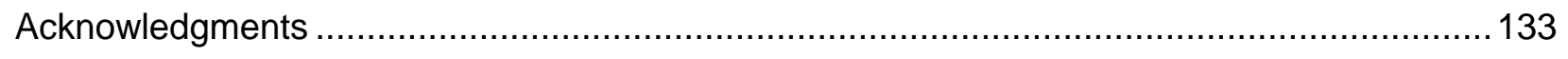

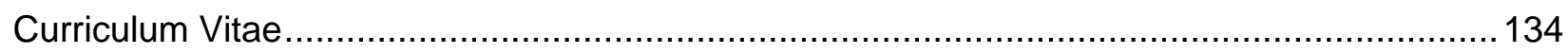

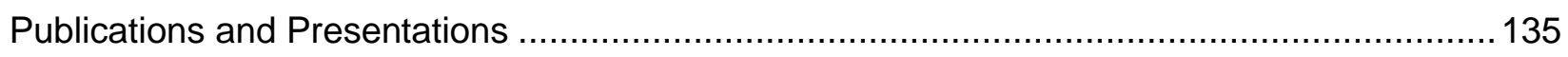

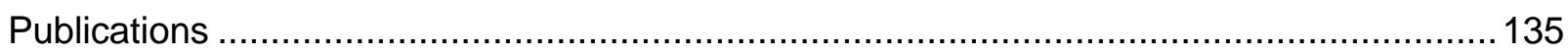

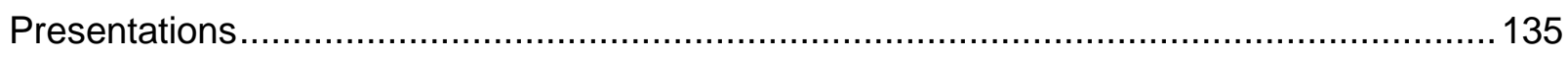

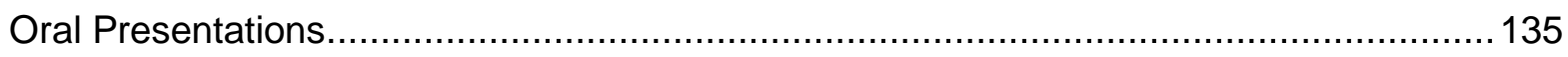

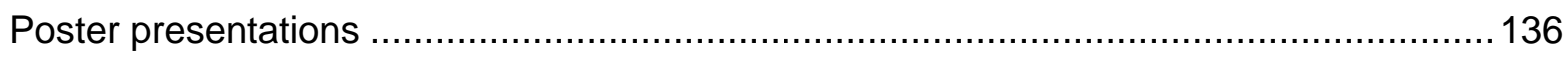

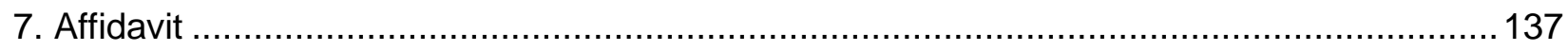




\section{List of tables}

Table 1: List of somatic cells and generated cell lines. * already established lines in the lab of K.

Streckfuß-Bömeke prior to project start

Table 2: Primers for PCR

Table 3: Primary antibodies - pluripotency

Table 4: Primary antibodies - germ layer

Table 5: Primary antibodies - cardiac

Table 6: Secondary antibodies used for IF and flow cytometry

Table 7: Components for cell culture

Table 8: Medias and supplements

Table 9: Chemicals and solutions

Table 10: Devices and machines

Table 11: Disposable items

Table 12: Software

Table 13: Components for RT-PCR

Table 14: Temperature for RT-PCR

Table 15: Components for PCR

Table 16: Conditions for PCR

Table 17: Setup for qPCR

Table 18: Temperature, duration, and cycle setup for the BioRad qPCR system

Table 19: Temperature, duration, and cycle setup for the 7900 HT qPCR system

53

Table 20: Recruited patients and healthy probands and underlying conditions 


\section{List of figures}

Figure 1: Normal cardiac physiology in human cardiomyocytes $\quad 9$

Figure 2: $\quad$ Schematic G-protein signaling in human cardiomyocytes $\quad 10$

Figure 3: Use of iPSCs in medical research 20

Figure 4: Schematic view of the used differentiation protocol 49

Figure 5: Reprogramming of somatic cell into iPSCs 65

Figure 6: $\quad$ iPSCs show pluripotency marker expression on protein level $\quad 66$

Figure 7: $\quad$ iPSC can be differentiated into all three germ layers in vitro and in vivo 68

Figure 8: $\quad$ Cardiac differentiations of iPSCs 70

Figure 9: $\quad$ Stress induction in iPSC-CMs by addition of high catecholamine 72 dosages

Figure 10: Multi-electrode arrays uncover electrical differences between 74 CTRL- and TTS-iPSC-CMs

Figure 11: Measurement of field potential durations (FPD) and frequency corrected field potential durations (FPDcF) with multi-electrode arrays uncover no significant differences between CTRL and TTS-iPSC-CMs.

Figure 12: Measurements of conduction velocities with multi-electrode arrays showed basal differences but no changes upon catecholamine stimulation.

Figure 13: Sample $\mathrm{Ca}^{2+}$ imaging recordings of CTRL-iPSC-CMs and TTS-IPSC-CMs.

Figure 14: $\quad \mathrm{Ca}^{2+}$ measurements of CTRL- and TTS-iPSC-CMs reveal 80 calcium handling differences.

Figure 15: Calcium spark analysis 82

Figure 16: Engineered heart muscles uncover an enhanced sensitivity 84 towards Iso of TTS-iPSC-CMs.

Figure 17: TTS-iPSC-CMs show lipid accumulation and differentially expressed lipid transporters. 
Figure 18: Influence of selective $\beta$-inhibition on electrical behavior of CTRL- and TTS-iPSC-CMs.

Figure 19: $\quad$ Selective $\beta$-inhibition with CGP and ICI uncover different roles of $\beta_{1-}$ and $\beta_{2}$-ARs in CTRL and TTS-iPSC-CMs.

Suppl. Fig. 1:T50 of all used CTRL- and TTS-iPSC-CMs 109

Suppl. Fig. 2:T50 and rise time in of CTRL-and TTS-iPSC-CMs under the 110 influence of CGP and/or ICI

Suppl. Fig. 3:Spark properties all used CTRL- and TTS-iPSC-CMs 


\section{Summary}

Takotsubo syndrome (TTS) is a severe acute heart disease presumably caused by high catecholamine levels resulting in wall motion abnormalities of the left ventricle. Despite the severe phenotype of TTS, the exact disease mechanisms remain elusive. This project aims to model TTS with the help of induced pluripotent stem cell (iPSC) derived cardiomyocytes (iPSC-CMs) on a patient-specific level. To achieve this goal, 4 TTS patients and 3 control probands were recruited. Human fibroblasts or peripheral blood mononuclear cells (PBMCs) were reprogrammed to iPSCs using integration-free methods. The resulting iPSCs exhibited typical iPSC-morphology and expressed specific pluripotency-related genes on messenger RNA (mRNA) and protein level. The cells could be readily differentiated into cells from all three different germ-layers in vitro and in vivo. IPSCs were successfully differentiated to iPSC-CMs with purity over $80 \%$ and expressing cardiac markers like cTnT and $\alpha$-actinin.

To mimic a TTS event, iPSC-CMs were treated with increasing concentrations of catecholamines such as epinephrine (Epi) or isoproterenol (Iso). The used high catecholamine levels did not induce significant levels of apoptosis, therefore excluding acute cytotoxicity. To test electrophysiological outcomes in the iPSC-CMs, beating rate and regularity were analyzed by using the multi-electrode array (MEA) system. MEA measurements uncovered electrical stunning for iPSC-CMs stimulated with rising levels of catecholamines. TTS-iPSC-CMs were more vulnerable to this effect. The remaining TTS-iPSC-CMs showed a stronger frequency increase when stimulated with Iso than CTRL-iPSC-CMs.

Functioning calcium $\left(\mathrm{Ca}^{2+}\right)$ cycling is of utmost importance in cardiomyocytes since $\mathrm{Ca}^{2+}$ is a key player in excitation-contraction coupling and $\mathrm{Ca}^{2+}$ abnormalities are involved in many heart diseases. TTS-iPSC-CMs demonstrated differences in $\mathrm{Ca}^{2+}$ cycling compared to CTRL-iPSC-CMs on multiple levels: Under basal conditions and after Iso stimulation, times to $50 \%$ decay $\left(T_{50}\right)$ were shorter and rise times were longer in TTS-iPSC-CMs in 
comparison to CTRL-iPSC-CMs. CTRL-iPSC-CMs showed a higher spark frequency at higher Iso levels $(1 \mu \mathrm{M})$, but overall, there was no difference in total $\mathrm{Ca}^{2+}$ leakage.

To analyze TTS on the tissue level, engineered heart muscles (EHM) were generated and used as a 3D-model. When challenged with Iso, the TTS-iPSC-EHMs showed a significantly higher sensitivity compared to CTRL-iPSC-EHMs as shown by a lower halfmaximal effective concentration $\left(E_{50}\right)$. After $24 \mathrm{~h}$ Iso pre-stimulation, the CTRL-iPSCEHMs exhibiting a stronger $\mathrm{EC}_{50}$ increase than TTS-iPSC-EHM, thereby exhibiting stronger receptor desensitization. Additionally, beating frequency changes after $24 \mathrm{~h}$ Iso stimulation were higher in TTS-iPSC-EHM compared to CTRL-iPSC-EHM, which is in line with the reported frequency differences in the 2D MEA measurements. Interestingly, TTSiPSC-EHM showed a lower force development than CTRL-iPSC-EHM without catecholamine stimulation.

Since adrenergic stimulation results in positive inotropic and chronotropic effects of the heart and thereby regulates lipid metabolism in cardiomyocytes, lipid droplets were quantified using Oil Red O. The TTS-iPSC-CMs exhibited higher lipid accumulation in comparison to CTRL-iPSC-CMs, suggesting a role of lipotoxicity in the disease.

The selective $\beta$-adrenergic receptor ( $\beta$-AR) blockers CGP ( $\beta_{1}$-AR blocker) and ICI ( $\beta_{2}-$ AR blocker) were used to uncover the roles of different $\beta$-ARs in CTRL- and TTS-iPSCCMs. In CTRL-iPSC-CMs, $\beta_{2}$-AR blocker alone was enough to reduce an Iso-dependent frequency increase and an Iso-dependent $T_{50}$ and rise time decrease, thus underpinning the role of the $\beta_{2}$-ARs in CTRL-iPSC-CMs. In TTS-iPSC-CMs, $\beta_{1}$-AR blockage abolished Iso-dependent $T_{50}$ and rise time reduction, which was not the case for $\beta_{2}-A R$ blockers and therefore underpins an important role of $\beta_{1}$-AR in TTS-iPSC-CMs.

In conclusion, these results demonstrate that CTRL- and TTS-iPSC-CMs behave differently with respect to catecholamine sensitivity, $\mathrm{Ca}^{2+}$ handling, lipid accumulation, electrophysiology, and $\beta$-AR preferences. This behavior hints towards a genetic predisposition in TTS, lowering the threshold for a TTS attack. 


\section{Abbreviations}

\begin{tabular}{|c|c|}
\hline a-SMA: & $\alpha$-smooth muscle actin \\
\hline$\beta$-Arr: & $\beta$-Arrestin \\
\hline$\beta-A R:$ & $\beta$-Adrenergic receptor \\
\hline AC: & Adenylate cyclase \\
\hline ACE: & Angiotensin converting enzyme \\
\hline AFP: & $\alpha-1-$ Fetoprotein \\
\hline ALB: & Albumin \\
\hline ALP: & Alkaline phosphatase \\
\hline BSA: & Bovine serum albumin \\
\hline $\mathrm{Ca}^{2+}:$ & Calcium \\
\hline cAMP: & Cyclic adenosinemonophophate \\
\hline cDNA: & Complementary DNA \\
\hline CHIR: & CHIR99021 \\
\hline CICR: & Calcium-induced calcium release \\
\hline CM: & Cardiomyocyte \\
\hline CTRL: & Control \\
\hline DAPI: & 4',6-Diamidino-2-phenylindole \\
\hline DMEM: & Dulbecco's modified Eagle medium \\
\hline DNA: & Desoxyribonucleic acid \\
\hline
\end{tabular}


E8:

Essential 8

EB: $\quad$ Embryoid body

$E_{50}$ : Half maximal effective concentration

ECG: $\quad$ Electrocardiogram

EHM: $\quad$ Engineered heart muscle

Epi: $\quad$ Epinephrine

ESC: $\quad$ Embryonal stem cell

FITC: $\quad$ Fluorescein isothiocyanate

FPD: $\quad$ Field potential duration

FPDcF: $\quad$ Frequency corrected field potential duration

FRET: $\quad$ Förster resonance energy transfer

GRK: $\quad$ G-protein coupled receptor kinase

GSK3: $\quad$ Glycogen synthase kinase 3

hESC: $\quad$ Human embryonic stem cells

IF: Immunofluorescence

Iso: $\quad$ Isoproterenol

iPSC: $\quad$ Induced pluripotent stem cell

iPSC-CM: $\quad$ iPSC-derived cardiomyocyte

iPSC-EHM: $\quad$ iPSC-derived engineered heart muscle

IWP: $\quad$ Inhibitor of Wnt production

KOSR: $\quad$ Knock-out serum replacement

MACCE: $\quad$ Major adverse cardiac and cerebrovascular events

MEA: $\quad$ Multi-electrode array 


\begin{tabular}{|c|c|}
\hline MEF: & Mouse embryonic fibroblasts \\
\hline MI: & Myocardial infarction \\
\hline $\min :$ & Minute \\
\hline miRNA: & Micro RNA \\
\hline mRNA: & Messenger RNA \\
\hline MTG: & Monothioglycerol \\
\hline NCX: & $\mathrm{Na}^{+} / \mathrm{Ca}^{2+}$ Exchanger \\
\hline NEAA: & Non-essential amino acids \\
\hline NR4A1: & Nuclear receptor subfamily 4 group A member 1 \\
\hline OCT4: & Octamer-binding transcription factor 4 \\
\hline PBS: & Phosphate-buffered saline \\
\hline PCR: & Polymerase chain reaction \\
\hline PDE: & Phosphodiesterase \\
\hline PDE4: & Phosphodiesterase 4 \\
\hline PFA: & Paraformaldehyde \\
\hline $\mathrm{Pl}:$ & Propidium iodide \\
\hline PKA: & Protein kinase A \\
\hline PLN: & Phospholamban \\
\hline PSC: & Parthenogenetic stem cells \\
\hline qPCR: & Quantitative polymerase chain reaction \\
\hline RNA: & Ribonucleic acid \\
\hline ROS: & Reactive oxygen species \\
\hline RPMI: & Roswell Park Memorial Institute \\
\hline
\end{tabular}




$\begin{array}{ll}\text { RT-PCR: } & \text { Reverse transcription PCR } \\ \text { RYR2: } & \text { Ryanodine receptor } 2 \\ \text { SERCA: } & \text { Sarcoplasmic/endoplasmic reticulum calcium ATPase } \\ \text { SNP: } & \text { Single nucleotide polymorphism } \\ \text { Sox2: } & \text { SRY-Box transcription Factor } 2 \\ \text { SR: } & \text { Sarcoplasmatic reticulum } \\ \text { STEMI: } & \text { ST-segment elevation myocardial infarction } \\ \text { SYN: } & \text { Synaptophysin } \\ \text { T50: } & \text { Time to } 50 \% \text { decay } \\ \text { TH: } & \text { Tyrosine hydroxylase } \\ \text { TTS: } & \text { Thiazotsubo Syndrome } \\ \text { TZV: } & \text { Thivin }\end{array}$




\section{Introduction}

\subsection{Takotsubo syndrome}

\subsubsection{Clinical presentation of Takotsubo syndrome}

Takotsubo syndrome is a recently described severe cardiac disease. It was first described in Japan in 1990 by Sato (Sato, 1990). Initially, only described in Japan, it was soon shown to be present worldwide (Desmet et al., 2003; Wittstein et al., 2005). Since then, it has become a more and more recognized cardiac health problem (Ghadri et al., 2018a). Typically, TTS is triggered by emotional or physical stress and is most prevalent in elderly women. It is characterized by left ventricular wall motion abnormalities of transient nature (Hurst et al., 2010; Templin et al., 2015). In 82\% of cases, the apical part of the left ventricle shows prominent dyskinesia and the basal parts of the ventricle show hyperkinesia (Templin et al., 2015). Additionally, there are some more uncommon representations of TTS with basal hypokinesia or medial hypokinesia described (Templin et al., 2015). The wall motion abnormalities give rise to the typical ventricular shape in TTS, showing apical ballooning. This very unique form reminded the first describers in Japan of a Takotsubo, a Japanese octopus trap with a shape similar to the typical TTS ventricle (Sato, 1990). The prevalence of TTS is not exactly known. However, TTS was diagnosed in about $2 \%$ of acute coronary syndrome suspect cases (Gianni et al., 2006), making up to $0.02 \%$ of overall hospitalization in the USA (Deshmukh et al., 2012).

To explain the underlying pathophysiology in TTS, many theories have been developed. Most of them agree upon the causative nature of high catecholamine levels in patients. Indeed, Wittstein et al. quantified the levels of catecholamines in TTS and MI patients and were able to show that the levels in TTS were at least twice as high in TTS than in MI (Wittstein et al., 2005). Furthermore, there are case reports where TTS is triggered after iatrogenic epinephrine (Epi) injection (Geppert et al., 2010; Zubrinich et al., 2008). TTS triggered by pheochromocytoma, a catecholamine-producing tumor, is another piece of

evidence towards catecholamines as the leading cause of TTS (Kim et al., 2010; Schmidt et al., 2017; Sharkey et al., 2015; Zieleń et al., 2010). Moreover, the local release of 
catecholamines by sympathetic neurons was shown to be increased in 5 TTS cases analyzed by Kume and colleagues (Kume et al., 2008). Notably, while catecholamines are the widest accepted explanation, $\beta$-blockers were not necessarily protecting patients from recurrent TTS attacks (Isogai et al., 2016; Sharkey et al., 2010). However, Ueyama and colleagues were able to readily prevent apical ballooning by beta-blocker pre-treatment in a rat model of TTS (Ueyama et al., 2002). Izumi et al. could provoke a TTS-like phenotype in cynomolgus monkey by Epi injection and were able to ameliorate the phenotype by using a one-time injection of the beta-blocker metoprolol (Izumi et al., 2009).

The wall motion abnormalities cause a significant drop in cardiac ejection fraction, which can be life-threatening. In consequence, patients suffer from dyspnea and chest pain and exhibit cardiac damage marker elevations including troponin and brain natriuretic peptide (Templin et al., 2015). Additionally, ST-segment elevation in the electrocardiogram (ECG) was on a similar level, as observed in myocardial infarction (MI) (Ghadri et al., 2018a; Templin et al., 2015). Moreover, additional ECG abnormalities such as ST-segment depression, T-wave inversion, and QTc prolongation can be present (Ghadri et al., 2018a). After the sudden onset of symptoms, the wall motion abnormalities recover over time without additional intervention (Templin et al., 2015). This might contribute to the fact that briefly after its first description, TTS outcome was considered mild (Prasad et al., 2008). However, the opposite is actually true: very severe complication as ventricular thrombi (Icli et al., 2016), ventricular rupture (Jaguszewski et al., 2012), left ventricular outflow tract obstruction (El Mahmoud et al., 2008), pulmonary edema (Bharathi et al., 2016), cardiogenic shock (Templin et al., 2015) and life-threatening arrhythmias (Stiermaier et al., 2015) can occur in TTS patients and demand for their close observation during hospitalization until full recovery (Ghadri et al., 2018b). The recently observed inhospital mortality rate of TTS from 3.7\% (Templin et al., 2015) to 4.2\% (Brinjikji et al., 2012) is comparable to that of MI with around 5\% (Templin et al., 2015). It is of note that while men are less likely to develop TTS, they harbor a significantly higher risk of inhospital death of $8.4 \%$ (Brinjikji et al., 2012). Unfortunately, after recovery from TTS, additional TTS attacks may occur with a probability of $1.8 \%$ per patient-year (Templin et al., 2015) or $5 \%$ overall in other studies (Sharkey et al., 2010), suggesting a certain vulnerability of the affected persons. It is noteworthy that after recovery from a TTS event, 
the long-term mortality (Stiermaier et al., 2016; Tornvall et al., 2016) and adverse cardiovascular event rates are as high as in patients who survived a MI (Templin et al., 2015).

Until today, there is no targeted treatment of TTS available as prospective randomized clinical trials are missing (Ghadri et al., 2018b). Therefore, TTS is mainly treated like acute coronary syndrome (Ghadri et al., 2018b). Patients are administered aspirin, heparin, $\beta$ blockers, diuretics, nitroglycerin, and angiotensin-converting enzyme (ACE) inhibitors while being monitored closely (Ghadri et al., 2018b; Sharkey et al., 2010). Additional, nonconventional drugs like the $\mathrm{Ca}^{2+}$ sensitizer levosimendan have been used in a small study (Santoro et al., 2013). In some cases, pacing devices become necessary to counteract arrhythmias (Ghadri et al., 2018b; Peters and Klein, 2012). Therefore, TTS is a severe cardiac disease and needs intensive research to find out the underlying pathology mechanisms and potentially helpful drugs.

The aforementioned clinical features were making it hard to distinguish TTS from Mls and thus going in line with the late description of the disease. Importantly, in contrast to a MI, there is no coronary vessel occlusion present in TTS. Consequently, a MI is ruled out during TTS diagnostics via coronary angiography with left ventriculography. To improve the diagnostic procedure of TTS, the Mayo Clinic criteria (Prasad et al., 2008) have been developed, which include several criteria to define TTS: transient wall motion abnormalities, which reach beyond the territory of a single coronary artery, the presence of new electrical abnormalities and absence of a coronary artery occlusion or plaque rupture (Prasad et al., 2008). Since then, many more diagnostic catalogs have been published, which include the possible coexistence of neurologic disorders (Ghadri et al., 2018a). Lately, a set of circulating micro RNAs (miRNA), namely miR-1, miR-16, miR-26a and miR-133a, have been found to differentiate TTS from healthy controls and STsegment elevation myocardial infarction (STEMI) patients. Unfortunately, the specificity was only around $70 \%$. However, this approach may help in the diagnostic procedure of TTS (Jaguszewski et al., 2014). 


\subsubsection{Trigger of Takotsubo attacks}

The cause of TTS remained elusive for some time. The link towards catecholamines was established by observing typical triggers for TTS. A link between TTS and stressful events such as natural disasters or the death of a loved one was observed in about $27 \%$ of TTS cases (Templin et al., 2015), giving the disease the nickname broken heart syndrome to reflect this. Consequently, after connecting TTS to stressful events, researchers found immensely increased catecholamine levels in patients with TTS (Wittstein et al., 2005). Interestingly, $28.5 \%$ of TTS cases show no obvious trigger (Templin et al., 2015). Notably, TTS is not only triggered by stressful emotional events, but it is also triggered by physical injury, mainly brain injury, which accounts for $36 \%$ of the TTS cases, with one prominent example being subarachnoid hemorrhage (Templin et al., 2015). In addition, stroke has been reported to trigger TTS (Blanc et al., 2015; Scheitz et al., 2012). Furthermore, 7.7\% of subarachnoid hemorrhage patients also developed TTS in a patient collective of 371 patients (Inamasu et al., 2016). There is also a strong connection between TTS and epilepsy and it is thought that TTS is indeed responsible for a fraction of sudden cardiac deaths in epilepsy patients (Dupuis et al., 2012). Generally, TTS has a strong correlation with neurologic (27\%) and psychiatric disorders (42\%), emphasizing the role of the heartbrain axis in the disease (Templin et al., 2015; Hiestand et al., 2018). Indeed, anxiety was significantly more present in a TTS patient collective compared to a STEMI collective or neutral patient collective analyzed by Summers et al. (Summers et al., 2010). An investigation by Delmas et al. yielded similar results: TTS patients showed more anxiodepressive disorders and chronic psychological stress than acute myocardial syndrome patients (Delmas et al., 2013). The connection between TTS and neuropsychiatric disorders, which clearly increase the TTS risk, is currently under intensive studies and demonstrates that the brain indeed has a strong influence on the heart as also shown for $\mathrm{MI}$, which is more likely to occur in patients suffering from depression (Wu and Kling, 2016).

\subsubsection{Predisposing factors for TTS}


TTS is most common in postmenopausal women, with $89.8 \%$ of patients being women overall (Templin et al., 2015). The increased vulnerability of these particular groups gave rise to the theory that estrogen does not only play a role but could be protective against TTS (Ueyama et al., 2003). Estrogens have been shown to influence vasomotor activity by influencing NO levels (Sader, 2002). Moreover, estrogen reduced catecholamine responses in perimenopausal women (Komesaroff et al., 1999). In the setting of subarachnoid hemorrhage in women, low estrogen levels were associated with cardiac wall motion disturbances (Sugimoto et al., 2012). Additionally, Ueyama and colleagues showed that estrogen ameliorated TTS in a model of ovariectomized rats (Ueyama et al., 2003). El-Battrawy and colleagues demonstrated in an iPSC-CM model that estradiol suppressed a catecholamine-caused action potential duration prolongation and reduced a catecholamine-based reactive oxygen species (ROS) production (El-Battrawy et al., 2018).

However, not only women but also men fall victim to the disease and the nature of the stressful events can be heterogeneous, as indicated by a TTS case of a fan during a stressful soccer match (Fijalkowski et al., 2013). Surprisingly, while most people tend to experience strong emotional stress at some point in their life, TTS is not regularly developed, arguing for an increased vulnerability of a fraction of the population, which indicates genetic or epigenetic risk factors. In support of a genetic component of TTS, several cases of TTS have been found in one family (Cherian et al., 2007). In support of these findings, Tak et al. reported a case of two sisters with TTS (Tak et al., 2018). Ikutomi et al. also reported two menopausal sisters with TTS suffering from TTS attacks just one year apart (Ikutomi et al., 2014). Another publication also reported TTS in two sisters aging 44 and 52 (Pison et al., 2004). The striking common ground of two sisters is logical given the age and gender distribution of TTS and its relatively recent discovery. Following the rationale of familial TTS cases, some groups found single nucleotide polymorphisms (SNPs), which are associated with TTS. An interesting SNP, which was found in the protein BAG3, inhibits the binding of BAG3 mRNA to the activating miRNA-371 (d'Avenia et al., 2015). This causes a reduction in BAG3 levels making the cells more prone to stressful events such as catecholamine flooding. Another of these SNPs is located in the G-protein receptor kinase 5 (GRK5), which is responsible for the deactivation of the 
catecholaminergic $\beta$-AR (Spinelli et al., 2010). Further research into $\beta$-AR signaling was done by Sharkey et al., who analyzed different mutations in $\beta$-ARs in TTS patients and found no differences between patients and healthy controls (Sharkey et al., 2009). Researching the interplay between $\beta$-ARs, subarachnoid hemorrhage and cardiac injury and dysfunction, Zaroff and colleagues found that the (Arg/Arg) combination of $\beta_{1}-A R$ Arg389Gly SNP in combination with $\alpha_{2}-A R$ deletion at amino acid positions 322-325 had a higher likelihood of reduced ejection fractions in observed patients (Zaroff et al., 2006). Another study compared $\beta$-AR variants between 61 TTS and 109 control cases and found a higher frequency of the same $\beta_{1}$-variant at amino acid position 389 as Zaroff et al. and a lower occurrence of Gln/Gln at the $\beta_{2}$-variant at amino acid position 27 in TTS (Vriz et al., 2011). A study from 2018 discovered heterogenous copy number variants of RBFOX1, GPC5, KCNRG, CHODL and GPBP1L1 in TTS cases (Lacey et al., 2018). Underpinning the heterogeneous nature of the SNPs found, some of these studies contradicted the findings of GRK5 and BAG3 polymorphisms in TTS (Figtree et al., 2013; Mattsson et al., 2018). Additionally, all studies have a relatively small sample size and therefore offer only limited insight. In summary, none of the discovered SNPs can fully explain all TTS cases. However, the number of SNPs found and familial clustering of some cases suggest a genetic component contributing to the pathogenesis of TTS.

One interesting factor in TTS is the epigenetic influence. It was shown that withdrawal of estrogen causes epigenetic changes (Zhao et al., 2010), which could, in theory, be a risk factor for the disease. Estrogen also has regulatory roles in catecholamine release and catecholamine effects in cardiomyocytes (Bupha-Intr and Wattanapermpool, 2006; Eskin et al., 2003; Li et al., 2000a; Ranki et al., 2001).

Notably, TTS patients have been recently associated with a high prevalence of malignant diseases (Girardey et al., 2016; Sattler et al., 2017, 2018). Therefore, it was already speculated that both diseases might be connected to a common source, which could be catecholamine excess (Sattler et al., 2018). This could very well contribute to the negative long-term outcome in TTS patients (Girardey et al., 2016). 


\subsubsection{A variety of hypotheses try to explain the TTS phenotype}

While high serum-level of catecholamines may trigger different disease entities, the exact mechanisms of how exposure to excessive catecholamine levels cause a TTS attack is widely unknown. Animal models and observations in patients have led to several theories.

As the wall motion abnormalities are not localized in an area nourished by one coronary artery, the occlusion of such a large vessel is unlikely to be the underlying cause. However, some groups think that microvascular spasms could cause such wall motion abnormalities (Galiuto et al., 2010). Galiuto and colleagues showed that adenosine injection during acute TTS events improved ventricular wall motion, thereby suggesting the involvement of microvascular vasoconstriction in the TTS etiology (Galiuto et al., 2010). Furthermore, slow coronary blood flow was observed in several TTS cases (Abdelmoneim et al., 2009; Vitale et al., 2016). It was demonstrated that vascular spasms can be caused by emotional stress (Lacy et al., 1995), which is also a major trigger of TTS. Another approach towards a vascular explanation was undertaken by Uchida et al., observing increased apoptosis in endothelial cells in individuals with TTS (Uchida et al., 2010). Additionally, endothelin-1 was found to be increased in TTS (Jaguszewski et al., 2014). Furthermore, endothelin-1 was found to cause vasoconstriction at high levels (Kiowski et al., 1991).

Paur et al. suggested a prominent role of the $\beta_{2}-\mathrm{AR}$ in TTS and provided evidence in a sophisticated mouse model. They postulate a switch from an activating $\mathrm{G}_{s}$ protein to an inhibiting $G_{i}$ protein triggered by high catecholamines levels (Paur et al., 2012). They also state that the $\beta_{2}$-ARs are more prominent in the apical part of the heart compared to the basal part, thereby explaining the specific shape of a TTS ventricle (Paur et al., 2012). To trigger TTS, Paur et al. injected a high bolus dosage of catecholamines into wildtype rats and found their ventricular function decreased. TTX, a blocker for $G_{i}$ signaling, was able to ameliorate this effect (Paur et al., 2012). It is of note that physiological studies in dogs found higher levels of norepinephrine in the basal part and less in the apical part of the left ventricle (Pierpont et al., 1984). This could outbalance the different AR densities. In 
line with Paur et al., Heubach and colleagues demonstrated that Epi activated $\mathrm{G}_{\text {s }}$ and $\mathrm{G}_{\mathrm{i}}$ proteins in mice hearts (Heubach et al., 2004).

Other groups including Shao et al. suggested that lipotoxicity might play a major role in the pathophysiology and show a strong lipid accumulation in their mouse model where they injected high dosages of catecholamines into the mice (Shao et al., 2013). Furthermore, the lipid transporters and lipid-associated proteins were different in catecholamine-treated mice compared to untreated mice. They also showed that TTS patient serum alone is sufficient to suppress the electrical activity of cardiomyocytes in vitro (Shao et al., 2013). This last finding, in part, contradicts the microvascular spasm theory as the sole pathophysiological mechanism as the cardiomyocyte suppression also worked in vitro without any vessels.

Additionally, oxidative stress might play a role in TTS. In this regard, Zhang et al. showed that $\mathrm{H}_{2} \mathrm{~S}$ can be protective in a TTS rat model by reducing NAPD oxidase and, ultimately, ROS production (Zhang et al., 2017). Additionally, in an iPSC-CM setting, Iso concentrations of $1 \mathrm{mM}$ were able to induce increased ROS production in the observed cells (El-Battrawy et al., 2018).

\subsection{Cardiac physiology}

\subsubsection{Action potential and cardiomyocyte contraction}


To understand TTS, it is important to investigate human cardiac physiology. In the heart, cardiac cells receive an action potential from the cardiac conduction system, which causes voltage-gated sodium channels to open, causing an influx of sodium ions depolarizing the cardiomyocytes (Figure 3). At a certain level of depolarization, voltage-gated L-type $\mathrm{Ca}^{2+}$ channels are opening while at the same time, the sodium channels are inactivated.

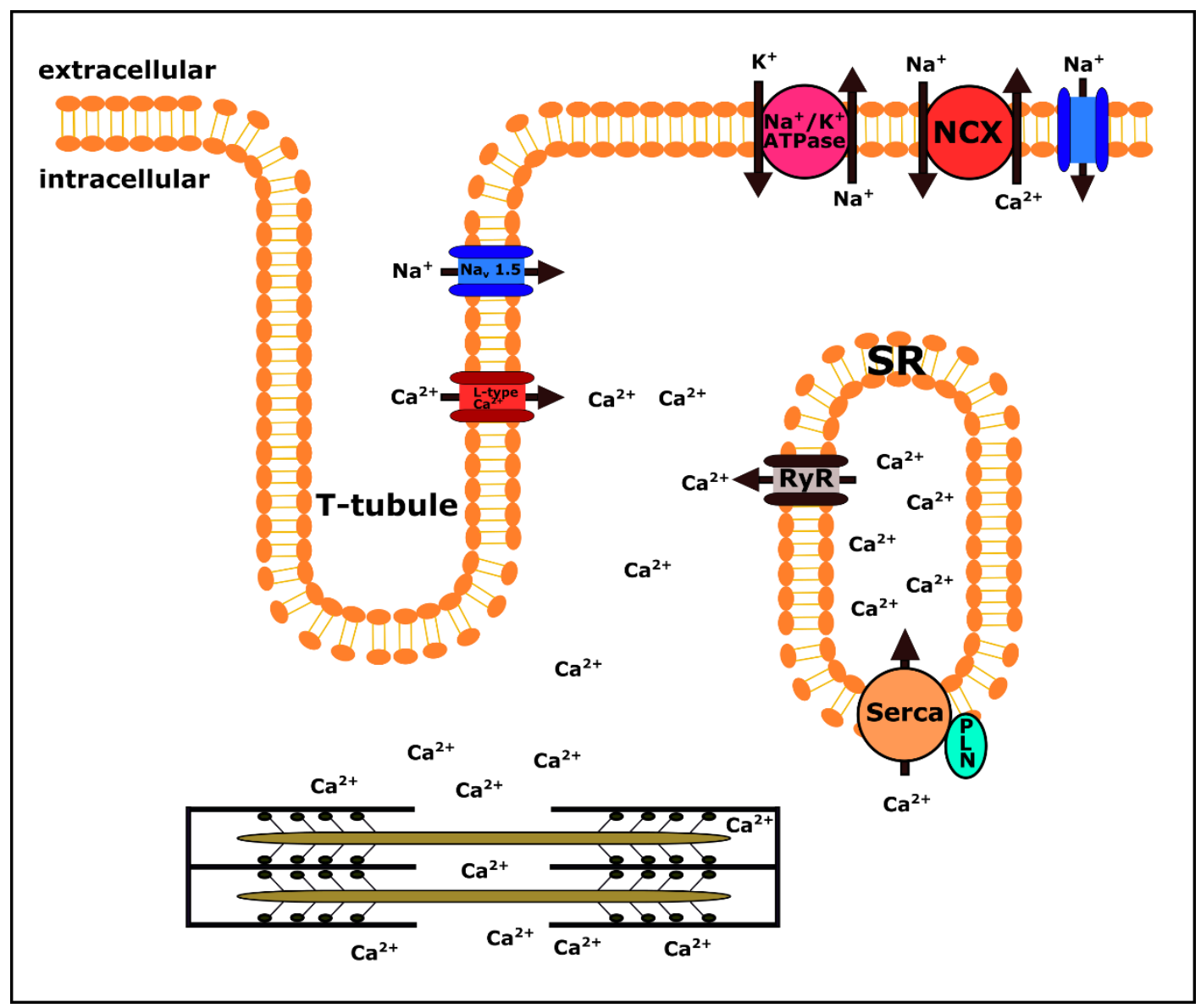

Figure 1: Normal cardiac physiology in a human cardiomyocyte. Sodium channels are opened upon a depolarizing signal and further depolarize the cardiomyocyte, which triggers calcium $\left(\mathrm{Ca}^{2+}\right)$ release from L-type calcium channels. This calcium triggers calcium-induced calcium release (CICR) from ryanodine receptor 2 (RyR2) from the sarcoplasmic reticulum (SR), which in turn enables contraction. After contraction, calcium is pumped back to the sarcoplasmic reticulum (SR) by the SERCA, which is tightly regulated by phospholamban (PLN). Part of the calcium is pumped back to extracellular space by the $\mathrm{Na}^{+} / \mathrm{Ca}^{2+}$ exchanger (NCX).

The newly generated $\mathrm{Ca}^{2+}$ influx keeps the cellular action potential at a plateau and triggers the calcium-induced release of even more $\mathrm{Ca}^{2+}$ ions by the ryanodine receptor 2 (RyR2), which is called calcium-induced calcium release (CICR) (Bers, 2002). The strongly increased $\mathrm{Ca}^{2+}$ levels in the cytoplasm of the cells resulted in binding of $\mathrm{Ca}^{2+}$ to 
troponin $\mathrm{C}$. As a consequence, troponin $\mathrm{C}$ changes its confirmation and subsequently releases myosin. Myosin can then, assisted by ATP, interact with actin to generate a power stroke and, therefore, muscle contraction.

During diastole and after contraction, $\mathrm{Ca}^{2+}$ is pumped back to the sarcoplasmic reticulum (SR) by the sarcoplasmic/endoplasmic reticulum $\mathrm{Ca}^{2+}$ ATPase (SERCA) and the extracellular space with the help of the $\mathrm{Na}^{+} / \mathrm{Ca}^{2+}$ exchanger (NCX) (Figure 3). The contraction is terminated by the reduction of $\mathrm{Ca}^{2+}$ levels, while the cell starts to relax. During this phase, the cellular potassium channels open and potassium is released to the extracellular space, therefore repolarizing the cardiomyocyte (Bers, 2002).

\subsubsection{The $\beta$-adrenergic system in cardiomyocytes regulates beating activity}

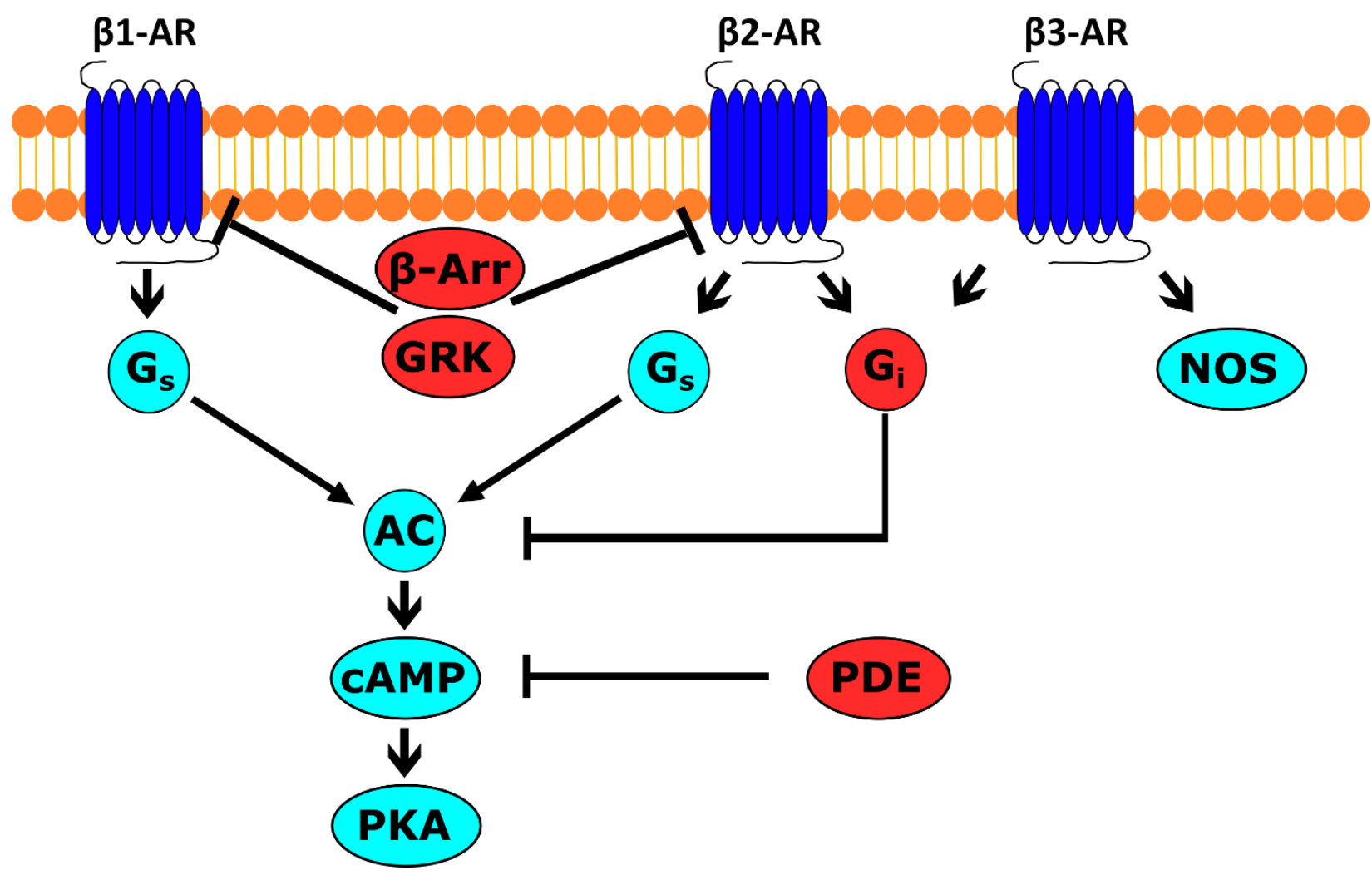

Figure 2: Schematic G-protein signaling in human cardiomyocytes. Activation of $\beta_{1^{-}}, \beta_{2}$-adreno receptors causes the activation of activating $G_{s}$ proteins. These proteins then activate the adenylate cyclase (AC). The AC in turn produces CAMP, which then activates protein kinase $A$ and further targets. The activation of $G_{i}$ proteins by $\beta$-ARs of the type $2 / 3$ is inhibiting the $G_{s}$ 
signaling through inhibition of AC. PDEs cause a breakdown of cAMP and therefore regulate cAMP signal strength. $\beta_{3}-A R s$ additionally activate nitric oxide synthases (NOS) producing nitric oxide enabling protein kinase $G$ signaling. The activity level of the $\beta$-AR is controlled by the GRK proteins, which phosphorylate the receptors. Picture modified from (Lohse Martin J. et al., 2003)

The process of cardiac contraction is tightly regulated by catecholamines, which are activating cellular $\beta_{1-}, \beta_{2}-$ and $\beta_{3}-A R s$. Catecholamines are released by sympathetic neurons in a local way and by the adrenal medulla in a more systemic way. Through this system, the brain has a strong influence on the heart. It is known that stroke patients often present with systolic and diastolic dysfunction and arrhythmias at least in part caused by catecholamine surges (Manea et al., 2015). Arrhythmias can damage the brain, for example, by thromboembolism, thereby creating a heart-brain axis (van der Wall and van Gilst, 2013). Therefore it is relatable that TTS is often triggered by dysfunction of the brain including subarachnoid hemorrhage (Banki et al., 2005). The released catecholamines produce a positive inotropic, positive dromotropic, positive chronotropic, positive lusitropic, and positive bathmotropic response in the heart (Bers, 2002). The $\beta_{1}$ - and $\beta_{2}-$ ARs release an activating $G_{s}$ protein, which activates the adenylate cyclase (Figure 2). The adenylate cyclase produces cyclic adenosine monophosphate (cAMP). The cAMP activates protein kinase $A(P K A)$, which in turn phosphorylates important proteins for cellular excitation and contraction like the L-type $\mathrm{Ca}^{2+}$ channel, the ryanodine receptor (RyR) and phospholamban (PLN) (Spadari et al., 2018). Normally, PLN inhibits the SERCA pump. A Phosphorylation of PLN stops its inhibiting activity and therefore results in an increased SERCA activity (Bers, 2002; Li et al., 2000b). The elevated SERCA activity causes faster reuptake of $\mathrm{Ca}^{2+}$ into the SR (positive chronotropic) and causes an increased loading of the SR with $\mathrm{Ca}^{2+}$, therefore enabling higher $\mathrm{Ca}^{2+}$ releases during systole (positive inotropy) (Bers, 2002). $\beta_{2}$ - and $\beta_{3}-A R s$ additionally activate $G_{i}$ proteins, which inhibit the AC, therefore suppressing cAMP response (Spadari et al., 2018). $\beta_{3}-A R s$ also activate nitric oxide synthases (NOS), thereby producing nitric oxide (NO), causing cGMP production and leading to protein kinase G activation (Cannavo and Koch, 2017; Trappanese et al., 2015). Protein kinase G phosphorylates cellular targets like L-type $\mathrm{Ca}^{2+}$ channels and seems to be cardioprotective (Cannavo and Koch, 2017). However, of cardiac $\beta$-ARs, only a minor fraction is made of $\beta_{3}-A R s$, giving the $\beta_{1}-A R s$ and $\beta_{2}-A R s$ more consideration in disease settings (Cannavo and Koch, 2017; Spadari et al., 2018). 
To control the activity of the $\beta$-ARs, G-protein coupled receptor kinases (GRK) phosphorylate the ARs, causing an internalization and shut off the signal. Furthermore, $\beta$ Arrestin is recruited to phosphorylated $\beta$-ARs and physically blocks receptor G-protein coupling (Fertig and Baillie, 2018; Krupnick and Benovic, 1998). Phosphodiesterases (PDE) shut down the CAMP signal by hydrolyzing cAMP and are recruited towards $\beta$-ARs by $\beta$-arrestin (Fertig and Baillie, 2018).

\subsection{Pluripotent stem cells}

\subsubsection{Multipotent stem cells are found in the human body}

The prospect of using primary human cells for regeneration and research has always been a dream of medical scientists. This dream was out of range for a very long time since human cells are not easy to come by and maintain in culture. Stem cell-like cells in the human body have long been known. In the human body, these so-called multipotent stem cells are critically important to maintain homeostasis by replacing damaged cells. A good example of such cells are hematopoietic and gut cells (Takashima et al., 2013). A hallmark of these cells is that they can divide indefinitely and produce a certain set of differentiated cells, for example, goblet cells (Takashima et al., 2013) or blood cells like erythrocytes and immune cells like B-cells or monocytes for bone hematopoietic stem cells (RodriguezFraticelli et al., 2018). While these cells can be isolated and cultured for some time, they only give rise to limited types of cells.

\subsubsection{Embryonic stem cells}

It is not surprising that in science and medicine, pluripotent or totipotent cells are of special interest as they can produce all cells of the body for pluripotent cells or all cells of the body and the cells of the extra-embryonal tissue for totipotent cells. Therefore, they are very useful for regenerative approaches. However, such cells were hard to obtain for a long time. In 1981, Evans and Kaufmann isolated cells from the inner cell mass of mouse 
blastocysts and were able to keep them in culture in an undifferentiated state (Evans and Kaufman, 1981). It turned out that these cells gave rise to all cells in the mouse body and were therefore regarded as pluripotent (Evans and Kaufman, 1981). They were named embryonic stem cells (ESC). Years later, in 1998, human embryonic stem cells (hESCs) were isolated by the team of Thomson and co-workers These cells were also pluripotent and were used to produce tissues from all three germ-layers (Thomson et al., 1998). Since then, hESCs have been used worldwide to create disease models (Halevy and Urbach, 2014) of Turner syndrome (Urbach and Benvenisty, 2009), spinal muscular atrophy (Wang et al., 2013), Rett syndrome (Li et al., 2013), Huntington's disease (Lu and Palacino, 2013; Ruzo et al., 2018) and the long-QT syndrome (Bellin et al., 2013). They have also been used in trials for regenerative medicine including neural (Adler et al., 2019) or cardiac regeneration (Chong et al., 2014). Unfortunately, the production of these cells goes hand in hand with the destruction of human embryos, which is very controversial debated under ethical considerations (Holm, 2015), and their use was therefore limited in some countries such as Germany (Kiechle, 2008).

\subsubsection{Alternative approaches to pluripotency}

To overcome the problems associated with hESCs, researchers were looking for alternative sources of pluripotent stem cells in adults. In 2006, Guan et al. were able to generate spermatogonial stem cells (SSCs) from mouse testis tissue. These cells were pluripotent and could be generated from adult tissue (Guan et al., 2006). They also showed that these cells could be differentiated into neurons (Streckfuss-Bömeke et al., 2009) and cardiomyocytes (Guan et al., 2007). An alternative approach to generate pluripotent cells with less ethical issues is treating oocytes with activating agents, thereby creating haploid parthenogenetic stem cells (PSCs) (Mai et al., 2007; Revazova et al., 2007; Sagi et al., 2016; Wang et al., 2018). Didié et al. demonstrated the ability of murine PSC to be differentiated into CMs just like ESCs and produced force-generating tissue, which was integrated into the recipient's hearts (Didié et al., 2013; Yang et al., 2015). Due to their nature, the PSCs only possess a limited amount of different MHC molecules, 
making them easier to match to potential recipient's immune system and are therefore a suitable candidate for a stem cell bank for future medical applications (Wang et al., 2018).

\subsubsection{Induced pluripotent stem cells - a major breakthrough}

Finally, in 2006 Yamanaka and colleagues showed that pluripotent cells can be produced from normal body cells by the addition of certain factors (Takahashi and Yamanaka, 2006). In an intensive screening approach of 24 different factors, where one factor was removed, and reprogramming efficiency was assessed, they identified KLF4, OCT4, SOX2 and C-MYC as sufficient to reprogram mouse fibroblasts to iPSCs. These cells were self-renewable and able to differentiate into tissue of all three germ-layers (Takahashi and Yamanaka, 2006). Subsequently, in 2007 Takahashi and colleagues demonstrated that human fibroblasts could be reprogrammed to iPSCs (Takahashi et al., 2007). They again used OCT4, KLF4, SOX2, and C-MYC in a retroviral vector to reprogram the cells. The produced cells turned out to be self-renewing and were able to differentiate into a variety of cells and tissues, therefore being pluripotent. In sum, the cells behaved similarly to hESCs (Takahashi et al., 2007). Thomson and colleagues were able to show nearly at the same time that human iPSCs can also be produced by transduction of OCT3/4, SOX2, LIN28, and NANOG, thereby introducing two new reprogramming factors, LIN28 and SOX2 (Yu et al., 2007). These cells overcame the limitations of previously known cells and the discovery of their reprogramming was a major medical breakthrough valued with the Nobel Prize 2012.

To eliminate the influence of remaining transgene expression or disruption of gene expression by transgene integration, researchers aimed for the use of non-integrating reprogramming methods. They used Sendai viruses (Fusaki et al., 2009), adenoviruses (Zhou and Freed, 2009), plasmid transfection (Okita et al., 2013), mRNA reprogramming (Mandal and Rossi, 2013), and even protein-based reprogramming (Kim et al., 2009; Zhou et al., 2009). These different reprogramming methods showed great differences in reprogramming efficiency dependent on the cell types used and also exhibited great efficiency differences when creating iPSCs from different patients. Neither integration-free 
methods such as mRNA-reprogramming (0.0-1.89\% efficiency) or episomal plasmids $(0.02-0.16 \%)$ nor integrating methods such as retroviruses $(0.02 \%-0.06 \%)$ tested by Goh et al. showed consistently better performance than the other in a feeder-free reprogramming approach (Goh et al., 2013). After the proof of principle of iPSC generation from fibroblasts, a wide range of cell types was successfully reprogrammed, including keratinocytes (Aasen and Izpisúa Belmonte, 2010), PBMCs (Isogai et al., 2018; Loh et al., 2009; Okita et al., 2013), and adipose-derived stem cells (Qu et al., 2012; Sugii et al., 2011). However, it must be stated that the reprogramming efficiencies were rather low at the beginning (Goh et al., 2013; Takahashi et al., 2007). Huebscher et al. reported that the reprogramming efficiencies are dependent on the source, with PBMCs showing the lowest efficiency (Hübscher et al., 2019). To achieve higher rates of reprogramming, different substances known to interact with epigenetics have been tested and used for enhanced reprogramming. One example is valproic acid, an HDAC inhibitor, which enhanced reprogramming efficiency by a factor of 100 in experimental conditions (Huangfu et al., 2008). Another example is sodium butyrate, which greatly enhanced reprogramming in several cell types (Hubbard et al., 2014; Zhang and Wu, 2013; Zhang et al., 2011b). Recently, optimization efforts were very successful, as demonstrated by Kogut and colleagues who reprogrammed fibroblasts with mRNAs and miRNA-367/302, generating 4019 iPSC colonies from 500 plated fibroblasts (Kogut et al., 2018). Additionally, researchers try to achieve a factor-free reprogramming strategy with the help of small molecules. In the mouse setting, there has been substantial progress towards this goal by replacing all factors with small molecules (Hou et al., 2013) thereby creating integration free and defined iPSCs.

\subsubsection{The Yamanaka factors trigger a complex pluripotent network activation in cells}

The main factors in the pluripotency network factors are the genes OCT4 and SOX2 (Li and Belmonte, 2017; Nichols et al., 1998). OCT4 itself is regulated by SOX2 on a transcriptional level (Li and Belmonte, 2017; Masui et al., 2007). NANOG, while not essential, helps to keep cells in an undifferentiated state. Cells without NANOG 
expression can remain pluripotent. They are, however, unable to differentiate into germ cells (Chambers et al., 2007). KLF4 seems to be involved with chromatin organization and long-range control of factors such as OCT4 (Di Giammartino et al., 2019; Li and Belmonte, 2017; Wei et al., 2013). MYC is important in maintaining proliferation during reprogramming and cells with low MYC levels are prone to cell death. Additionally, it silences genes associated with the somatic fate of the cell (Díaz-Díaz et al., 2017; Rand et al., 2018; Takahashi and Yamanaka, 2016).

It is currently believed that reprogramming takes part in two steps (Takahashi and Yamanaka, 2016). During the first step, tissue-specific factors are repressed, and early pluripotency markers are expressed. This step is connected with a huge epigenetic barrier and thus can be enhanced by epigenetic modulators including HDAC inhibitors such as sodium butyrate (Liang et al., 2010; Takahashi and Yamanaka, 2016). In the second step, the late pluripotency genes are upregulated in a more controlled way than the early genes (Buganim et al., 2012; Takahashi and Yamanaka, 2016). To explain the low efficiency of reprogramming, several models, including an elite cell model where only a small portion of a cell population is in principle suitable for reprogramming have been proposed (Takahashi and Yamanaka, 2016). However, researchers now think that in principle, all cells can be reprogrammed and that the key lies in the induction of pluripotency. A slight imbalance of the different reprogramming factors, which is likely to occur, might tilt the fragile balance towards a different cellular fate, thereby inhibiting reprogramming efforts (Takahashi and Yamanaka, 2016). Wernig and colleagues demonstrated this quite elegant by using a doxycycline-inducible cassette with the four Yamanaka factors, which achieved a very balanced expression of these factors (Wernig et al., 2008). The induction by doxycycline caused a very high percentage of reprogramed cells (Wernig et al., 2008). Concerning desoxyribonucleic acid (DNA) methylation and epigenetics, it is of note that iPSCs keep the methylation of their parental cells for some time (Bar-Nur et al., 2011). Therefore, it can be problematic to differentiate these cells properly for some time. However, after some passages, this epigenetic memory is lost and the cells can be readily differentiated into different cells. On the other hand, remaining epigenetic memory can 
ease the differentiation process towards the parent-cell lineage (Bar-Nur et al., 2011; Ma et al., 2014; Noguchi et al., 2018).

\subsection{6 iPSCs can be differentiated towards a wide range of cells}

To fully unleash the potential of pluripotent cells, sophisticated differentiation protocols had to be developed. With the knowledge of human developmental signaling pathways and screening approaches, successful differentiation of iPSCs towards cardiomyocytes (Zhang et al., 2009), neurons (Chang et al., 2011; Egawa et al., 2012; Lee et al., 2009; Lu et al., 2012), skeletal muscles (Borchin et al., 2013), immune cells (Kennedy et al., 2012), hepatocytes (Liu et al., 2011; Rashid et al., 2010) and many more functional cell types were achieved.

\subsubsection{Cardiac differentiation of iPSCs}

To obtain optimal results during disease modeling and regeneration, it is of utmost importance to achieve a good differentiation and maturation of the cells. In the case of cardiomyocytes, the first approach was to spontaneously differentiate the cells in a random way and select the cardiomyocyte manually by excising beating areas (Zhang et al., 2009). The yield of cardiomyocytes from this method was rather poor and the method itself is rather time-consuming. Nevertheless, these embryoid body (EB) methods have been careful and systematically improved, resulting in tremendously increased yields (Mummery et al., 2012). That was achieved by adding extra cells in coculture to enhance differentiation (Mummery et al., 2003) or the addition of proteins or small molecules (Burridge et al., 2007; Freund et al., 2008; Kattman et al., 2011; Mummery et al., 2012).

An alternative to EB-based methods was introduced by 2D monolayer differentiation methods, which are easier to handle. To achieve a better differentiation, manipulation of the Wnt pathway, which is also involved in human development, was performed. The Wntpathway is firstly activated by CHIR99021 (CHIR) (A GSK3-inhibitor) to achieve mesoderm formation and then suppressed by inhibitor of Wnt production 2 (IWP2) to direct 
the mesoderm in a cardiac-specific way (Burridge et al., 2014). Additionally, Burridge et al. systematically reduced the additive B27 from 27 compounds to only three essential: ascorbic acid, recombinant albumin, and the chemically defined medium RPMI1640 (Burridge et al., 2014). With this method, robust differentiation is possible and relatively easy. To obtain an even higher purity, cardiomyocytes can be selected with a glucosedeprived medium containing lactate, as cardiomyocytes metabolize lactate as an energy source (Burridge et al., 2014). Using this approach, cardiomyocytes with a high purity of up to $95 \%$ TNNT2-positive cells can be produced (Burridge et al., 2014).

The differentiated pure cells, however, were still not mature, as was seen in the expression of ion channels and electrical behavior. This is very understandable as the human heart takes years to mature (Ahmed et al., 2020; Vreeker et al., 2014). To obtain more mature cells, different strategies were developed. Some groups used protein supplements and chemical molecules to enhance maturation, whereas others used special scaffolds and matrixes to achieve this goal. Naturally, the most obvious strategy is a longer maturation, which was used by Lundy et al., showing an improvement in contraction and $\mathrm{Ca}^{2+}$ handling after cultivation of 80-120 days versus 20-40 days (Lundy et al., 2013). Cardiomyocyte properties are also depending on the surface they are cultured on (Wang et al., 2011). Wang and co-workers showed that different nanogrooved substrates affected the orientation and contractile functions of cultured rat cardiomyocytes (Wang et al., 2011). Ruan and colleagues used mechanical stimulation to produce more mature human iPSCCMs (Ruan et al., 2016). Additionally, T3 was used in one study to boost maturation and caused stronger contraction force and improved $\mathrm{Ca}^{2+}$ handling (Yang et al., 2014). Further improvement was achieved by combining T3 with dexamethasone on a matrigel mattress, resulting in improved $\mathrm{Ca}^{2+}$ coupling and the presence of a T-tubule network in human iPSC-CMs (Parikh et al., 2017). Another approach is tissue culture, as demonstrated by Tiburcy et al. producing engineered heart muscle by combining iPSC-CMs with fibroblasts, thereby achieving force generation and $\mathrm{CM}$ form and connection comparable to neonatal cardiomyocytes (Tiburcy et al., 2017). Additionally to pure cardiomyocyte differentiation, researchers further aimed to produce certain subsets of cardiomyocytes such as ventricular cells, atrial cells, or pacemaker cells, since these cells are very useful in disease modeling or regenerative approaches (Cyganek et al., 2018). With the help of 
retinoic acid, cardiomyocytes of the atrial type could be produced from ESCs (Zhang et al., 2011a) and iPSCs (Cyganek et al., 2018). Weng and colleagues used a combination of BMP4, activin-A, and IWR-1 to obtain ventricular iPSC-CMs of purities of more than 90\% (Kolanowski et al., 2017; Weng et al., 2014). Nodal cardiomyocyte production is not yet as effective as ventricular cardiomyocyte generation (Kolanowski et al., 2017). However, NRG-1 $\beta$ and ErbB inhibitors were used successfully in the generation of nodallike cells (Zhu et al., 2010).

\subsubsection{Disease modeling with the help of iPSCs - a step towards future drug screenings}

Disease modeling is another important use of the new iPSC-technology with its unlimited cell source of different tissues. Figure 3 shows typical applications of iPSCs in this field. They can serve as an unlimited source of specialized cells used for disease modeling and subsequent drug or safety screenings. According to Avior and colleagues, for modeling human diseases with iPSCs, the disease of choice should fulfill some criteria: The disease should have high penetration, should be monogenetic, early-onset, and have a clear cellular phenotype (Avior et al., 2016). Consequently, complex genetic backgrounds, late onset of the disease and low penetration increase the difficulty of disease modeling (Avior et al., 2016). With the help of iPSCs-derived cells, many genetic and non-genetic diseases were modeled in vitro with differentiated iPSCs.

A growing number of diseases have already been modeled with the help of iPSC-derived neurons, such as Alzheimer's disease (Jones et al., 2017), Parkinson's disease (Ren et al., 2015), amyotrophic lateral sclerosis (Burkhardt et al., 2013; Egawa et al., 2012; Sances et al., 2016) and even diseases as schizophrenia (Da Silveira Paulsen et al., 2012; Paulsen et al., 2014). In the cardiology field, there are several known genetic diseases, which have been modeled with the help of iPSC-CMs. Some examples include catecholaminergic polymorphic ventricular tachycardia (Kujala et al., 2012; Zhang et al., 2013), long QT syndrome 1 (Moretti et al., 2010), 2 (Matsa et al., 2011) and 3 (Bellin et al., 2012; Davis et al., 2012), Leopard syndrome (Carvajal-Vergara et al., 2010), 
arrhythmogenic right ventricular cardiomyopathy (Ma et al., 2013), Brugada syndrome (Veerman et al., 2016), Barth syndrome (Dudek et al., 2013), anthracycline-induced cardiomyopathy (Burridge et al., 2016; Haupt et al., 2020), and familial dilated cardiomyopathy (Bellin et al., 2012; Streckfuss-Bömeke et al., 2017; Sun et al., 2012).

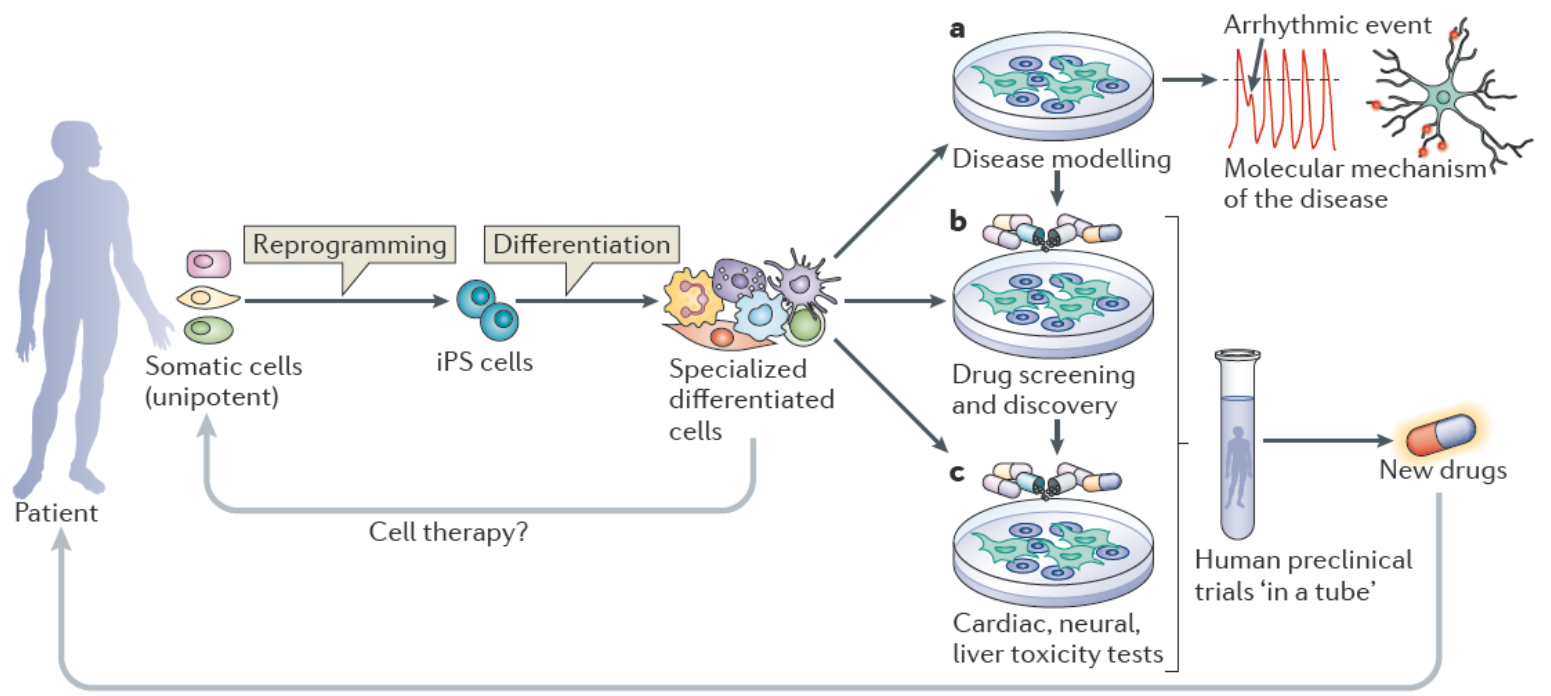

Figure 3: Use of iPSCs in medical research. A: Use of differentiated cells as a human disease model. The cells recapitulate the individual phenotype of the patient. B: These models can then be used for drug screenings. C: The differentiated cells can also be used as toxicity screening models in the pharmaceutic industry to improve preclinical trial quality and validity. (Bellin et al., 2012). License number provided by the Copyright Clearance Center: 4932720740448

Interestingly, also epigenetic diseases have been modeled. Sagie and colleagues were able to model immunodeficiency, centromere instability and facial anomalies syndrome, which is caused by a defect in a methyltransferase, causing a hypomethylation (Sagie et al., 2014).

These new disease models provide valuable insights into the pathophysiology of different diseases. Most importantly, these models provide the possibility to develop and test new therapies and drugs in a human setting (Figure 3).

\subsubsection{Role of iPSCs in the field of regenerative medicine}


It is no surprise that the possibility of an unlimited cell source of nearly any cell type triggered great hopes in the broad field of regenerative medicine. One big leap forward in the emerging field of regeneration was the cure of sickle cell anemia in mice by transplantation of bone marrow progenitor cells made from skin-derived iPSCs (Hanna et al., 2007). Furthermore, neuronal regeneration studies were performed in a monkey model of Parkinson's disease (Kikuchi et al., 2017). One especially promising therapeutic approach seems to be the regeneration of pancreatic islet cells to cure diabetes, which was shown to reconstitute insulin production in a mice diabetic model (Yabe et al., 2019).

Concerning cardiac regeneration, studies in larger animal models were completed lately by Shiba and colleagues who transplanted allogenic iPSC-derived cardiomyocytes in monkeys (Shiba et al., 2016). They show that the cells not only survive and couple to the host cardiomyocytes electrically but also that the infarcted hearts of the monkey treated with the produced cardiomyocytes show a better outcome than the untreated hearts. However, they also state that there are risks associated with their procedure in the form of ventricular arrhythmias, which occurred in their model (Shiba et al., 2016). Masumoto and co-workers used engineered cardiac tissue constructs composed of cardiomyocytes, endothelial cells, and vascular mural cells to ameliorate heart infarcts in rats (Masumoto et al., 2016). In 2012, Templin et al. demonstrated with elegant experiments using sodium iodide symporter labeling of cardiomyocytes injected into pigs, that cell populations can be traced in vivo for up to 15 weeks (Templin et al., 2012). Chong and colleagues injected one billion cardiomyocytes into infarcted monkey hearts and showed that the engrafted cells couple electrically with the host cells. The observed cells show a synchronized $\mathrm{Ca}^{2+}$ pattern. However, in many animals of the study, non-lethal arrhythmias were observed (Chong et al., 2014). Lately, more complex approaches emerge, for example, using a mesenchymal stem cell patch transplanted onto hearts supplemented by iPSC-CMs injection into rat hearts (Park et al., 2019). Menasché and colleagues used ESC-derived cardiac progenitors for clinical transplantation onto a human heart in a patient suffering from heart failure (Menasché et al., 2015). They showed that the transplanted patch was able to beat with the rest of the heart and no problems such as rejection or arrhythmias were reported in the study (Menasché et al., 2015). In a follow-up study, they were able to show that the transplanted cells did not form tumors or cause arrhythmias. Furthermore, 
an increased motion of the transplanted area was observed (Menasché et al., 2018). Jiang et al. injected human iPSC-CMs into infarcted hearts to ameliorate the myocyte loss, generating improved function (Jiang et al., 2020). Surprisingly, most of the injected cells were lost. However, there were some improvements, which have been thought to be connected to the endocrine activity of the injected cells (Jiang et al., 2020).

In many diseases, a gene correction is necessary before a regenerative approach can be considered. Some groups managed to correct the causative mutations in vitro with genome-editing methods. A good example of that is the correction of the $\alpha_{1}$-antitrypsin mutation with the help of zinc-finger nucleases by Yusa et al., which showed that future gene therapies are possible (Yusa et al., 2011). Lately, the correction of some monogenetic human diseases was performed in iPSCs with the help of CRISPR/Cas9 (Jacków et al., 2019; Yingjun et al., 2019; Yumlu et al., 2019). It was shown that genecorrected iPSC-based skin grafts generated from dystrophic epidermolysis bullosa patients showed no disease in mice (Jacków et al., 2019). Furthermore, $\beta$-thalassemia was corrected in iPSC cells from patients (Yingjun et al., 2019) with the result that the corrected and differentiated iPSCs were able to produce functional hemoglobin after transplantation in mice (Ou et al., 2016). Moreover, Hanses et al. demonstrated successful disease modeling and intronic CRISPR repair of the LZTR1 gene in an IPSC-CM system (Hanses et al., 2020). Additionally, Duchenne muscular dystrophy has been repaired using CRISPR in iPSC approaches (Li et al., 2014; Young et al., 2016).

One hurdle with cell transplantation is the immune response. Normally, autologous iPSCs or their differentiated cells should not cause any immune response. However, new studies suggest that there can be an immune response based on NK-cells (Dressel et al., 2010). In a different approach, Shiba et al. demonstrated that allogenic iPSC-CMs were successfully transplanted in a monkey MI model, thereby improving ventricular function (Shiba et al., 2016). Monkeys were treated with prednisolone and tacrolimus and no immune rejection was observed (Shiba et al., 2016). Morizane et al. demonstrated that immune suppression and MHC matching ameliorated the immune response towards iPSC-derived, transplanted dopaminergic neurons (Morizane et al., 2017). Interestingly, Badin and colleagues observed a different outcome when transplanting iPSC-based 
neuronal grafts in a Huntington's disease model in non-human primates (Badin et al., 2019). Despite the grafts being HLA matched, a rejecting immune response was observed. This response, however, was more delayed than in non-matched grafts (Badin et al., 2019).

\subsubsection{Importance of differentiated iPSCs for safety screenings}

Nowadays, iPSC-based cells are widely used to perform safety screenings during drug development. This method is evermore expanding in importance as it may replace animal screening models to a certain extend and are closer to human physiology than the aforementioned animal models. Until now, several screening platforms have been established, like the screening of cardioactive drugs for their influence on cardiac ion channels and, therefore possible arrhythmias (del Álamo et al., 2016). Another example is the work of Sharma and colleagues, who performed a high throughput screening of tyrosine kinase inhibitors for cardiotoxicity (Sharma et al., 2017). Today more and more sophisticated disease and drug screening models are developed. For example, it has become quite common to use 3D structures made from cardiomyocytes and fibroblasts as a modeling and therapeutic platform (Tiburcy et al., 2017). These EHM can provide very useful insights as they represent a more complex interplay between cells and cell types as can be seen in 2D cultures and therefore resemble the situation in the human heart in a more accurate way (Huebsch et al., 2016; Tiburcy et al., 2017). Furthermore, commercially available platforms have been developed, which employ several cell types made from iPSCs on a chip to enable organ-based drug screenings as drugs often influence several tissues. Automated or semi-automated screening systems for ion channels and electrical behavior of the cells supplement these systems and open up new possibilities in drug screening and help to improve safety pharmacology. As in EHM, several cell types, which contribute towards tissues, become more and more used in the iPSC field in the form of organoids, thus providing more accurate drug screening platforms. IPSC-derived brain organoids consist of different cell types like glia and neurons and exhibit network activity between neurons (Zafeiriou et al., 2020). Recently, Lee and coworkers developed murine cardiac organoids. The developing organoids were 
self-organized, consisted of atrial- and ventricular-like cells, and also contained endothelial cells (Lee et al., 2020). These organoids will greatly enhance drug screenings and disease model approaches in the future, as they can model the complex interplay of different cell types in organs.

\subsubsection{Limitations of iPSCs applications}

The new possibilities available by the use of iPSCs can advance the scientific and industrial field. However, some disadvantages exist. The production of iPSCs is costly and rather time-consuming, which is also true for their maintenance and differentiation. It can be argued that these cells do not exist in nature and will always be an artificial system. Dependent on the source cell type, there is also heterogeneity in stem cell gene expression, which can complicate the reproducibility of experiments and differentiation (Ghosh et al., 2010; Streckfuss-Bömeke et al., 2013). Furthermore, at the moment, most differentiation methods do not produce pure populations of cells (Burridge et al., 2014). It is unlikely that in vitro and in vivo differentiated cells will behave similarly in every situation. However, as mentioned earlier, differentiation and maturation protocols have been improved tremendously in the past.

The used iPSC models do provide single or multiple cell types and do not yet provide the context of a whole organic system, as normal animal studies can provide. This might be problematic, as diseases often cause complex interactions between different organ systems. Organoids made from iPSCs have been used to mimic organic tissues (Kawada et al., 2017; Lancaster et al., 2013). Furthermore, in iPSC models, there is always the need for well-characterized adequate controls to reduce variability, which are difficult to obtain. Ideally, isogenic controls with a corrected disease-causing mutation would be used (Bellin et al., 2012). 


\subsection{Aim of the thesis}

As TTS is a severe cardiovascular condition, this thesis aims to create a human iPSCbased model of TTS in a dish. To achieve that, several TTS patients and healthy control persons will be recruited and somatic cells like fibroblasts and PBMCs will be isolated. The obtained cells will be reprogrammed to iPSCs with integration-free methods. The newly produced iPSCs will be characterized to ensure their pluripotent characteristics. Subsequently, the iPSCs will be differentiated into functional human cardiomyocytes of high purity and used for disease modeling. For this TTS model, several hypotheses are made:

- First, TTS has at least a genetic component, thereby increasing the risk for affected person groups.

- Second, TTS can be triggered by high levels of catecholamines.

- Third, TTS can be modeled in a dish by stressing iPSC-CMs with catecholamines.

- Fourth, the in vitro TTS model can be used to uncover pathomechanisms and to test new drugs.

Based on these hypotheses, the following aims were explored:

- Generation and characterization of pluripotency of TTS- and CTRL-iPSC lines

- Differentiation of iPSCs into functional beating iPSC-CMs

- Analysis of TTS and CTRL-iPSC-CMs upon catecholamine treatment regarding apoptosis, electrical activity, $\mathrm{Ca}^{2+}$ homeostasis, and lipid accumulation in 2D iPSCCMs culture

- Analysis of catecholamine-sensitivity, desensitization and force of contraction in a 3D-engineered heart muscle model

- Identification of different $\beta$-AR sub-receptors important in the development of TTS 


\section{Material}

\subsection{Cells used in this study}

Table 1: List of somatic cells and generated cell lines. * already established lines in the lab of $\mathrm{K}$. Streckfuß-Bömeke prior to project start

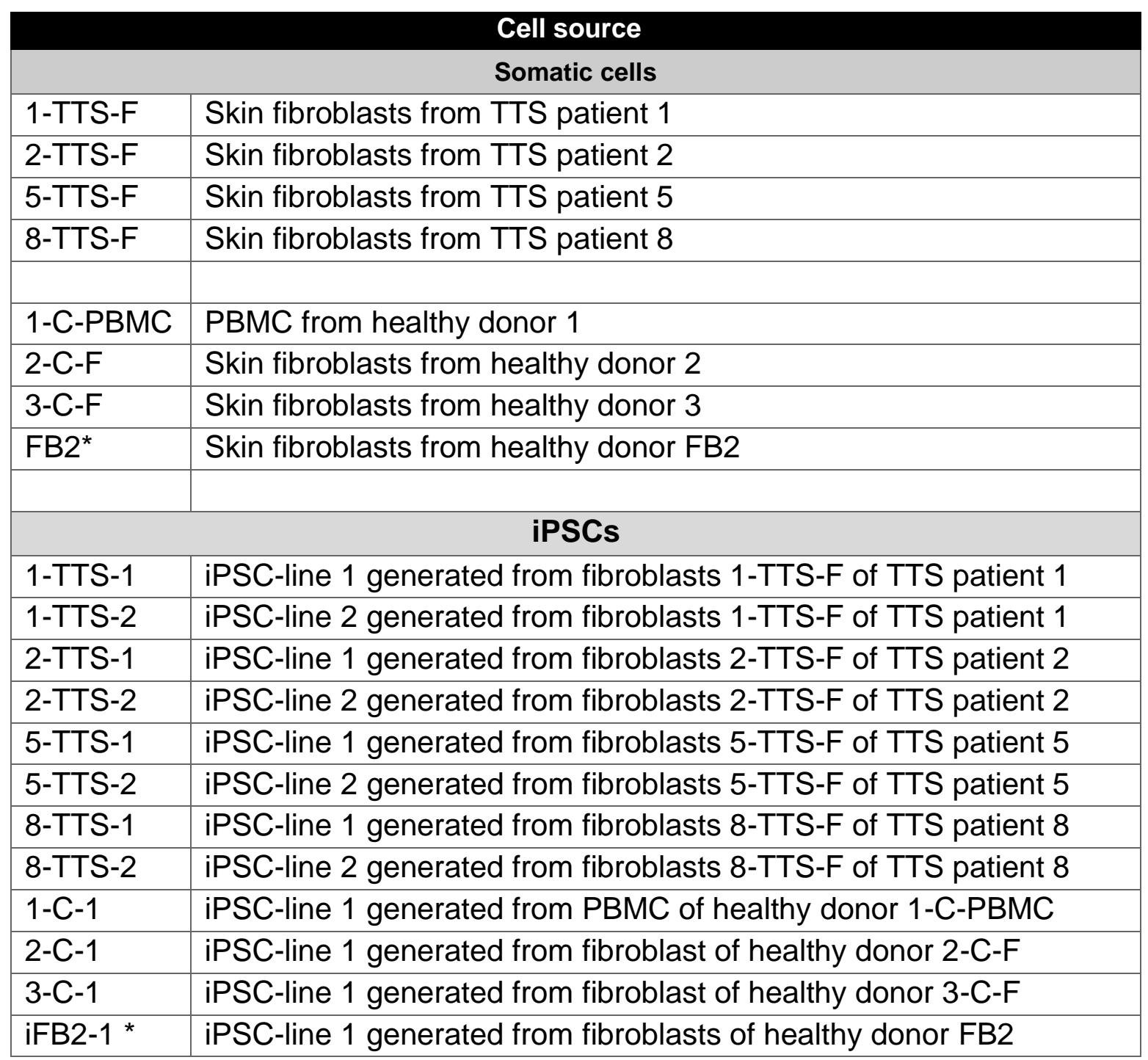

\subsection{Primer}


Primers for polymerase chain reaction (PCR) were designed by using the primer blast webpage from the National Center for Biotechnology Information.

Table 2: Primers for PCR

\begin{tabular}{|c|c|c|c|c|c|}
\hline $\begin{array}{l}\text { Gene } \\
\text { name }\end{array}$ & $\begin{array}{l}\text { Product } \\
\text { size (bp) }\end{array}$ & Forward primer sequence & $\begin{array}{l}\text { Reverse primer } \\
\text { sequence }\end{array}$ & $\begin{array}{c}\text { Annealing } \\
\text { temperature }\end{array}$ & Cycle \\
\hline GAPDH & 258 & $\begin{array}{l}\text { AGA GGC AGG GAT } \\
\text { GAT GTT CT }\end{array}$ & $\begin{array}{l}\text { TCT GCT GAT } \\
\text { GCC CCC ATG TT }\end{array}$ & $55^{\circ} \mathrm{C}$ & 34 \\
\hline OCT4 & 218 & $\begin{array}{l}\text { GAC AAC AAT GAA } \\
\text { AAT CTT CAG GAG A }\end{array}$ & $\begin{array}{l}\text { TTCTGGCGCCGG } \\
\text { TTACAGAACCA }\end{array}$ & $58^{\circ} \mathrm{C}$ & 36 \\
\hline NANOG & 164 & $\begin{array}{l}\text { AGT CCC AAA GGC } \\
\text { AAA CAA CCC ACT TC }\end{array}$ & $\begin{array}{l}\text { ATC TGC TGG } \\
\text { AGG CTG AGG } \\
\text { TAT TTC TGT CTC }\end{array}$ & $64^{\circ} \mathrm{C}$ & 30 \\
\hline LIN28 & 410 & $\begin{array}{l}\text { AGT AAG CTG CAC } \\
\text { ATG GAA GG }\end{array}$ & $\begin{array}{l}\text { ATT GTG GCT } \\
\text { CAA TTC TGT GC }\end{array}$ & $52^{\circ} \mathrm{C}$ & 38 \\
\hline sox2 & 437 & $\begin{array}{l}\text { ATG CAC CGC TAC } \\
\text { GAC GTG A }\end{array}$ & $\begin{array}{l}\text { CTT TTG CAC } \\
\text { CCC TCC CAT TT }\end{array}$ & $56^{\circ} \mathrm{C}$ & 43 \\
\hline GDF3 & 311 & $\begin{array}{l}\text { TTC GCT TTC TCC } \\
\text { CAG ACC AAG GTT } \\
\text { TC }\end{array}$ & $\begin{array}{l}\text { TAC ATC CAG } \\
\text { CAG GTT GAA } \\
\text { GTG AAC AGC } \\
\text { ACC }\end{array}$ & $54^{\circ} \mathrm{C}$ & 32 \\
\hline FOXD3 & 353 & $\begin{array}{l}\text { GTG AAG CCG CCT } \\
\text { TAC TCG TAC }\end{array}$ & $\begin{array}{l}\text { CCG AAG CTC } \\
\text { TGC ATC ATG AG }\end{array}$ & $61^{\circ} \mathrm{C}$ & 38 \\
\hline $\begin{array}{l}\text { MYH6 ( } \alpha- \\
\text { MHC) }\end{array}$ & 413 & $\begin{array}{l}\text { GTC ATT GCT GAA } \\
\text { ACC GAG AAT G }\end{array}$ & $\begin{array}{l}\text { GCA AAG TAC } \\
\text { TGG ATG ACA } \\
\text { CGC T }\end{array}$ & $60^{\circ} \mathrm{C}$ & 40 \\
\hline $\begin{array}{l}\text { MYH7 ( } \beta- \\
\text { MHC) }\end{array}$ & 101 & $\begin{array}{l}\text { AGACTGTCGTGGGCT } \\
\text { TGTATCAG }\end{array}$ & $\begin{array}{l}\text { GCCTTTGCCCTTC } \\
\text { TCAATAGG }\end{array}$ & $63^{\circ} \mathrm{C}$ & 30 \\
\hline ACTN1 & 291 & $\begin{array}{l}\text { AGG AGG AAG AAT } \\
\text { GGC CTG AT }\end{array}$ & $\begin{array}{l}\text { GAT GCA GTA } \\
\text { CTG GGC CTG AT }\end{array}$ & $60^{\circ} \mathrm{C}$ & 30 \\
\hline cTNT & 305 & $\begin{array}{l}\text { GAC AGA GCG GAA } \\
\text { AAG TGG GA }\end{array}$ & $\begin{array}{l}\text { TGA AGG AGG } \\
\text { CCA GGC TCT AT }\end{array}$ & $56^{\circ} \mathrm{C}$ & 26 \\
\hline
\end{tabular}




\begin{tabular}{|l|c|l|l|c|c|}
\hline NR4A1 & 130 & $\begin{array}{l}\text { GTG TGT GGG GAC } \\
\text { AAC GCT TC }\end{array}$ & $\begin{array}{l}\text { CCA CAG GGC } \\
\text { AGT CCT TGT T }\end{array}$ & $60{ }^{\circ} \mathrm{C}$ & 40 \\
\hline CD36 & 176 & $\begin{array}{l}\text { CAG TTC TCA ATC } \\
\text { TGG CTG TGG C }\end{array}$ & $\begin{array}{l}\text { AAC AGG GTA } \\
\text { CGG AAC CAA } \\
\text { ACT CA }\end{array}$ & $60{ }^{\circ} \mathrm{C}$ & 40 \\
\hline CPT1C & 169 & $\begin{array}{l}\text { GCT TTC AGC TGG } \\
\text { GCT ACT CA }\end{array}$ & $\begin{array}{l}\text { ACG ACA TGG } \\
\text { CAG TCG ACA TT }\end{array}$ & $60^{\circ} \mathrm{C}$ & 40 \\
\hline
\end{tabular}

\subsection{Plasmids}

For integration-free reprogramming, plasmids from Okita et al. were used (Okita et al., 2011):

pCXLE-hOCT3/4-shp53-F was a gift from Shinya Yamanaka (Addgene plasmid \# 27077; http://n2t.net/addgene:27077 ; RRID:Addgene_27077)

pCXLE-hSK was a gift from Shinya Yamanaka (Addgene plasmid \# 27078;

http://n2t.net/addgene:27078; RRID:Addgene_27078)

pCXLE-hUL was a gift from Shinya Yamanaka (Addgene plasmid \# 27080;

http://n2t.net/addgene:27080 ; RRID:Addgene_27080)

\subsection{Primary antibodies}

The antibodies used for immunofluorescence (IF) and fluorescence-activated cell sorting (FACS) are listed in the following tables.

Table 3: Primary antibodies - pluripotency

\begin{tabular}{|l|l|l|l|}
\hline Antigen & Source & Company & Concentration \\
\hline hLIN28 & Goat & R\&D; AF3757 & $1: 300$ \\
\hline SOX2 & Mouse & R\&D; MAB2018 & $1: 50$ \\
\hline hOCT3/4 & Goat & R\&D; AF1759 & $1: 40$ \\
\hline hNANOG & Goat & R\&D; AF1997 & $1: 200$ \\
\hline TRA-1-60 & Mouse & Ab16288 & $1: 200$ \\
\hline SSEA4 & Mouse & [MC813] ab16287 & $1: 200$ \\
\hline
\end{tabular}


Table 4: Primary antibodies - germ layer

\begin{tabular}{|l|l|l|l|}
\hline Antigen & Source & Company & Concentration \\
\hline$\alpha-S M A$ & Mouse & Sigma; A2547 & $1: 3000$ \\
\hline$\beta$-Tubulin & Mouse & BioLegend & $1: 2000$ \\
\hline hAFP & Rabbit & Dako & $1: 100$ \\
\hline
\end{tabular}

Table 5: Primary antibodies - cardiac

\begin{tabular}{|l|l|l|l|}
\hline Antigen & Source & Company & Concentration \\
\hline a-Actinin & Mouse & Sigma; A7811 & $1: 1000$ \\
\hline cTNT & Mouse & $\begin{array}{l}\text { Thermo Scientific; } \\
\text { MS-295-PADX }\end{array}$ & $1: 200$ \\
\hline
\end{tabular}

\subsection{Secondary antibodies}

Table 6: Secondary antibodies used for IF and flow cytometry

\begin{tabular}{|l|l|l|l|l|}
\hline Antigen & Fluorophore & Host & Company & Conc. IF \\
\hline $\begin{array}{l}\text { Rabbit } \\
\lg G(H+L)\end{array}$ & $\begin{array}{l}\text { Alexa Fluor } \\
647\end{array}$ & donkey & $\begin{array}{l}\text { life Technologies; } \\
\text { A31573 }\end{array}$ & $1: 1000$ \\
\hline $\begin{array}{l}\text { Rabbit } \\
\lg G(H+L)\end{array}$ & $\begin{array}{l}\text { Alexa Fluor } \\
555\end{array}$ & donkey & $\begin{array}{l}\text { life Technologies; } \\
\text { A31572 }\end{array}$ & $1: 1000$ \\
\hline $\begin{array}{l}\text { Goat IgG } \\
(\mathrm{H}+\mathrm{L})\end{array}$ & $\begin{array}{l}\text { Alexa Fluor } \\
555\end{array}$ & donkey & $\begin{array}{l}\text { life Technologies; } \\
\text { A21432 }\end{array}$ & $1: 1000$ \\
\hline $\begin{array}{l}\text { Mouse } \\
\lg G(H+L)\end{array}$ & $\begin{array}{l}\text { Alexa Fluor } \\
488\end{array}$ & donkey & $\begin{array}{l}\text { life Technologies; } \\
\text { A21202 }\end{array}$ & $1: 1000-1: 500$ \\
\hline $\begin{array}{l}\text { Mouse } \\
\lg G, \lg M \\
(H+L)\end{array}$ & $\begin{array}{l}\text { Alexa Fluor } \\
488\end{array}$ & goat & $\begin{array}{l}\text { life Technologies; } \\
\text { A10680 }\end{array}$ & $1: 500$ \\
\hline
\end{tabular}




\begin{tabular}{|l|l|l|l|l|}
\hline $\begin{array}{l}\text { Mouse } \\
\text { IgG+lgM }\end{array}$ & Cy3 & goat & Jackson & $1: 300 \mathrm{u}$ \\
$(\mathrm{H}+\mathrm{L})$ & & & ImmunoResearch; & $1: 600$ \\
& & & $115-165-068$ & \\
& & & LOT:124776 & \\
\hline
\end{tabular}

Phalloidin (Life Technologies; A34055) was used to stain F-actin and was coupled to the dye Alexa Fluor 555.

\subsection{Cell culture and media}

The following table shows additives used for cell culture:

Table 7: Components for cell culture

\begin{tabular}{|c|c|}
\hline Component & Supplier, catalog number \\
\hline $0.25 \%$ Trypsin-EDTA & Thermo Fisher Scientific \#25200056 \\
\hline $\begin{array}{l}\text { 4', 6-Diamidino-2-phenylindole } \\
\text { dihydrochloride (DAPI) }\end{array}$ & Sigma-Aldrich \#D9542 \\
\hline Albumin, human recombinant & Sigma-Aldrich, \#A02347 \\
\hline B-27 Serum-free supplement (50x) & $\begin{array}{l}\text { Thermo Fisher Scientific, } \\
\# 17504044\end{array}$ \\
\hline B-27 Supplement, minus insulin & Thermo Fisher Scientific \#A1895601 \\
\hline Basic fibroblast growth factor (bFGF) & PeproTech, \#100-18B \\
\hline $\begin{array}{l}\text { Bovine albumin fraction } \mathrm{V} \text { solution (BSA, } \\
7.5 \%)\end{array}$ & Thermo Fisher Scientific \#15260037 \\
\hline Calcium chloride dihydrate $\left(\mathrm{CaCl}_{2}\right)$ & Roth \#HN04 \\
\hline CGP 20712A & Sigma-Aldrich \#C231 \\
\hline CHIR99021 (CHIR) & Merck Millipore, \#361559 \\
\hline
\end{tabular}




\begin{tabular}{|l|l|}
\hline Collagenase B & $\begin{array}{l}\text { Worthington Biochemical, \#CLS- } \\
\text { AFB }\end{array}$ \\
\hline Collagenase IV & Worthington Biochemical, \#CLS-4 \\
\hline Cytotune 2.0 Sendai Kit & Life technologies: A16517 \\
\hline Dexamethasone & Sigma, D8893 \\
\hline Dimethylsulfoxide (DMSO) & Sigma-Aldrich, \#D2650 \\
\hline DMEM (Dulbecco's modified Eagle & Thermo Fisher Scientific, \\
medium) & \#11960044 \\
\hline DMEM/F12 & $\begin{array}{l}\text { Thermo Fisher Scientific, } \\
\text { \#31331028 }\end{array}$ \\
\hline DPBS (Dulbecco's phosphate-buffered & $\begin{array}{l}\text { Thermo Fisher Scientific, } \\
\text { \#14190169 }\end{array}$ \\
\hline Epinephrine hydrochloride & Sigma-Aldrich, \# E4642-5G \\
\hline Essential 8 Medium & $\begin{array}{l}\text { Thermo Fisher Scientific, } \\
\text { \#A1517001 }\end{array}$ \\
\hline Ethanol absolute & Th. Geyer, \#1025490 \\
\hline Fetal bovine serum (FBS) & Sigma-Aldrich, \#F7524 \\
\hline Erythropoietin & Life technologies, \#PHC2054 \\
\hline Ethylenediaminetetraacetic acid (EDTA) & Sigma-Aldrich \#E6758 \\
\hline Gelatine & Sigma-Aldrich, \#48720 \\
\hline Geltrex (Growth factor reduced) & $\begin{array}{l}\text { Thermo Fisher Scientific, } \\
\text { \#A1413302 }\end{array}$ \\
\hline Glucose & Sigma-Aldrich \#G8270 \\
\hline Hydrochloric acid (HCl) fuming 37 \% & Merck Millipore, \#100317 \\
\hline ICl 118,551 & Sigma-Aldrich \#I127 \\
\hline IGF1 & Peprotech \#AF-100-11 \\
\hline IL-3 & Life technologies, \#PHC0034 \\
\hline Insulin & Life technologies, \#12585014 \\
\hline Iscove's modified Dulbecco's medium with & Thermo Fisher Scientific, \\
\hline
\end{tabular}




\begin{tabular}{|c|c|}
\hline $\begin{array}{l}\text { Isoproterenol hydrochloride } \\
\text { Synonym: Isoprenaline hydrochloride }\end{array}$ & Sigma-Aldrich, \# I5627-5G \\
\hline Isopropanol & Merck Millipore \#109634 \\
\hline IWP2 & Merck Millipore, \#681671 \\
\hline Knock Out Serum & $\begin{array}{l}\text { Thermo Fisher Scientific, } \\
\# 10828028\end{array}$ \\
\hline L-Ascorbic acid 2-phosphate & Sigma-Aldrich, \#A8960 \\
\hline L-glutamine (200 mM, 100x) & $\begin{array}{l}\text { Thermo Fisher Scientific, } \\
\text { \#25030024 }\end{array}$ \\
\hline LB bouillon (Miller) & Merck \#1102850500 \\
\hline Mitomycin C & Serva Electrophoresis, \#29805.02 \\
\hline Monothioglycerol (MTG) & Sigma-Aldrich, \#M6145-25ML \\
\hline Non-essential amino acids (NEAA, 100x) & $\begin{array}{l}\text { Thermo Fisher Scientific, } \\
\# 11140035\end{array}$ \\
\hline $\begin{array}{l}\text { Penicillin-streptomycin solution (P/S) } \\
(100 x)\end{array}$ & $\begin{array}{l}\text { Thermo Fisher Scientific, } \\
\# 15140112\end{array}$ \\
\hline Polyfect & Qiagen \#301105 \\
\hline RPMI 1640 with HEPES with GlutaMAX & $\begin{array}{l}\text { Thermo Fisher Scientific, } \\
\# 724000021\end{array}$ \\
\hline $\begin{array}{l}\text { RPMI } 1640 \text { without HEPES without } \\
\text { Glucose }\end{array}$ & $\begin{array}{l}\text { Thermo Fisher Scientific, } \\
\# 11879020\end{array}$ \\
\hline Sodium DL-lactate solution $60 \%(\mathrm{w} / \mathrm{w})$ & Sigma-Aldrich, \#L4263 \\
\hline Sodium Selenite & Sigma, \#S5261 \\
\hline Stem cell factor (SCF) & Life technologies, \#PHC2115 \\
\hline TGF-b1 & Peprotech \#AF-100-21C \\
\hline Thiazovivin (TZV) & Merck Millipore, \#420220 \\
\hline Trypsin/EDTA (0.25\%) & Thermo Fisher Scientific, \#2520056 \\
\hline VEGF $_{165}$ & Peprotech \#AF-100-20 \\
\hline Versene Solution (0.48 mM EDTA) & $\begin{array}{l}\text { Thermo Fisher Scientific, } \\
\# 15040066\end{array}$ \\
\hline
\end{tabular}


Media and supplements and their preparation are stated in the following table:

Table 8: Medias and supplements

\begin{tabular}{|c|c|}
\hline Name & composition \\
\hline bFGF & $\begin{array}{l}100 \mu \mathrm{g} \text { bFGF dissolved in } 1 \mathrm{ml} \text { Tris }(5 \mathrm{mM}) \text { and stored at }-20^{\circ} \mathrm{C} \text {; } \\
\text { working solution: } 5 \mathrm{ng} / \mu \mathrm{l} \text {, diluted } 1: 20 \mathrm{in} 0.1 \% \mathrm{BSA} / \mathrm{DPBS}\end{array}$ \\
\hline $\begin{array}{l}\beta \text {-mercaptoethanol } \\
(100 x)\end{array}$ & $7 \mu \mathrm{l}$ diluted in $10 \mathrm{ml}$ DPBS and sterile filtered, stored at $4^{\circ} \mathrm{C}$ \\
\hline Blood medium & $\begin{array}{l}50 \% \text { IMDM } \\
50 \% \text { Ham's F12-Nutrient Mix } \\
5 \mathrm{mg} / \mathrm{mL} \text { albumin } \\
1 \times \text { lipid concentrate } \\
14 \mathrm{ng} / \mathrm{mL} \text { sodium selenite } \\
64 \mu \mathrm{g} / \mathrm{mL} \text { L-ascorbic acid 2-phosphate } \\
10 \mu \mathrm{g} / \mathrm{mL} \text { insulin, } 100 \mu \mathrm{gg} / \mathrm{mL} \text { transferrin } \\
1 \mathrm{mM} \mathrm{dexamethasone} \\
450 \mu \mathrm{M} 1 \text {-thioglycerol } \\
50 \mathrm{ng} / \mathrm{mL} \text { stem cell factor } \\
2 \mathrm{U} / \mathrm{mL} \text { erythropoietin } \\
40 \mathrm{ng} / \mathrm{mL} \text { IGF-I } \\
10 \mathrm{ng} / \mathrm{mL} \text { IL-3 }\end{array}$ \\
\hline BSA (1\%) & $1 \mathrm{ml}$ of $7.5 \%$ BSA added to $6.5 \mathrm{ml} \mathrm{DPBS}$, stored at $4{ }^{\circ} \mathrm{C}$ \\
\hline $\begin{array}{l}\text { Cardio } \quad \text { Culture } \\
\text { Medium }\end{array}$ & $\begin{array}{l}500 \mathrm{ml} \text { RPMI } 1640 \text { with HEPES with GlutaMAX, } 10 \mathrm{ml} \mathrm{B-27} \\
\text { supplement with insulin, stored at } 4{ }^{\circ} \mathrm{C}\end{array}$ \\
\hline $\begin{array}{l}\text { Cardio } \\
\text { Differentiation } \\
\text { Medium }\end{array}$ & $\begin{array}{l}500 \mathrm{ml} \text { RPMI } 1640 \text { with HEPES with GlutaMAX } \\
250 \mathrm{mg} \text { albumin (human recombinant) } \\
100 \mathrm{mg} \text { L-ascorbic acid } 2 \text {-phosphate } \\
\text { sterile-filtered and stored at } 4^{\circ} \mathrm{C}\end{array}$ \\
\hline $\begin{array}{l}\text { Cardio Digestion } \\
\text { Medium }\end{array}$ & $\begin{array}{l}80 \mathrm{ml} \text { Cardio Culture Medium } \\
20 \mathrm{ml} \text { FBS } \\
100 \mu \mathrm{l} \text { Thiazovivin (final concentration } 2 \mu \mathrm{M} \text { ) }\end{array}$ \\
\hline
\end{tabular}




\begin{tabular}{|c|c|}
\hline & stored at $4{ }^{\circ} \mathrm{C}$ \\
\hline $\begin{array}{l}\text { Cardio Selection } \\
\text { Medium }\end{array}$ & $\begin{array}{l}500 \mathrm{ml} \text { RPMI } 1640 \text { without HEPES without GlutaMAX } \\
2 \mathrm{ml} \text { lactate/HEPES ( } 1 \mathrm{M}) \\
250 \mathrm{mg} \text { albumin (human recombinant) } \\
100 \mathrm{mg} \text { L-ascorbic acid 2-phosphate } \\
\text { stored at } 4{ }^{\circ} \mathrm{C}\end{array}$ \\
\hline $\begin{array}{l}\text { CHIR99021 } \quad(12 \\
\text { mM) }\end{array}$ & $\begin{array}{l}5 \mathrm{mg} \text { CHIR99021 dissolved in } 0.894 \mathrm{ml} \text { DMSO } \\
\text { stored at }-20^{\circ} \mathrm{C}\end{array}$ \\
\hline $\begin{array}{l}\text { Collagenase } \quad B \\
(400 \mathrm{U} / \mathrm{ml})\end{array}$ & $\begin{array}{l}\text { dissolved in RPMI medium to a working solution of } 400 \mathrm{U} / \mathrm{ml} \\
\text { sterile-filtered and stored at } 4{ }^{\circ} \mathrm{C}\end{array}$ \\
\hline $\begin{array}{l}\text { Collagenase IV } \\
(200 \mathrm{U} / \mathrm{ml})\end{array}$ & $\begin{array}{l}\text { dissolved in DMEMD/F12 to a working solution of } 200 \mathrm{U} / \mathrm{ml} \text {, } \\
\text { sterile-filtered and stored at } 4{ }^{\circ} \mathrm{C}\end{array}$ \\
\hline $\begin{array}{l}\text { Cryopreservation } \\
\text { Medium (10 ml) }\end{array}$ & $\begin{array}{l}2 \mathrm{ml} \text { DMSO } \\
8 \mathrm{ml} \text { Essential } 8 \text { Medium } \\
20 \mu \mathrm{l} \mathrm{TZV}(2 \mathrm{mM}) \\
\text { stored at } 4{ }^{\circ} \mathrm{C} \text { for one week }\end{array}$ \\
\hline $\begin{array}{l}\text { Epinephrine } \\
\text { hydrochloride }\end{array}$ & $\begin{array}{l}109.8 \mathrm{mg} \text { were dissolved in } 10 \mathrm{~mL} \text { water and filtered sterile (50 } \\
\mathrm{mM} \text { stock solution) }\end{array}$ \\
\hline $\begin{array}{ll}\text { Essential } & 8 \\
\text { Medium } & \end{array}$ & $\begin{array}{l}500 \mathrm{ml} \text { Essential } 8 \text { basal Medium } \\
10 \mathrm{ml} \text { Essential } 8 \text { supplement }\end{array}$ \\
\hline Fetal bovine serum & heat-inactivated for $30 \mathrm{~min}$ at $56^{\circ} \mathrm{C}$ \\
\hline $\begin{array}{ll}\text { Feeder layer } \\
\text { Medium }\end{array}$ & $\begin{array}{l}84 \mathrm{ml} \text { DMEM } \\
15 \mathrm{ml} \text { FBS (heat-inactivated) } \\
1 \mathrm{ml} \text {-glutamine }\end{array}$ \\
\hline Freezing Medium & $18 \mathrm{ml}$ DMEM \\
\hline
\end{tabular}




\begin{tabular}{|c|c|}
\hline & $\begin{array}{l}5 \mathrm{ml} \text { FBS (heat inactivated) } \\
2 \mathrm{ml} \mathrm{DMSO}\end{array}$ \\
\hline Gelatine $(0.1 \%)$ & $\begin{array}{l}5 \mathrm{~g} \text { Gelatine dissolved in } 5 \text { I distilled water, autoclaved and stored } \\
\text { at } 4{ }^{\circ} \mathrm{C}\end{array}$ \\
\hline Geltrex & $\begin{array}{l}2 \mathrm{mg} \text { of geltrex aliquoted and stored at }-20^{\circ} \mathrm{C} \text {; dissolved in } 12 \mathrm{ml} \\
\text { cold DMEM/F12 before use }\end{array}$ \\
\hline $\begin{array}{l}\text { Human Embryonic } \\
\text { Stem Cell Medium } \\
\text { (hESC-Medium) }\end{array}$ & $\begin{array}{l}500 \mathrm{ml} \text { DMEM/F12 } \\
90 \mathrm{ml} \text { KnockOut Serum } \\
6 \mathrm{ml} \mathrm{NEAA} \\
6 \mathrm{ml} \beta \text {-Mercaptoethanol (100x) } \\
10 \mathrm{ng} / \mathrm{ml} \mathrm{bFGF} \mathrm{(freshly} \mathrm{added)} \\
\text { stored at } 4^{\circ} \mathrm{C}\end{array}$ \\
\hline $\begin{array}{l}\text { Human Fibroblast } \\
\text { Medium (HFBM) }\end{array}$ & $\begin{array}{l}\text { DMEM } \\
10 \% \text { heat-inactivated FBS } \\
1 \times \text { NEAA } \\
1 \times \text { L-glutamine } \\
1 \times \beta-M E \\
10 \mathrm{ng} / \mathrm{mL} \text { hbFGF (freshly added) }\end{array}$ \\
\hline $\begin{array}{l}\text { Iscove } \quad \text { Medium } \\
(100 \mathrm{ml})\end{array}$ & $\begin{array}{l}79 \mathrm{ml} \text { IMDM } \\
20 \mathrm{ml} \mathrm{FBS} \\
1 \mathrm{ml} \text { NEAA } \\
450 \mu \mathrm{M} \text { MTG (freshly prepared), stored at } 4{ }^{\circ} \mathrm{C}\end{array}$ \\
\hline $\begin{array}{l}\text { Isoproterenol } \\
\text { hydrochloride }\end{array}$ & $\begin{array}{l}247.7 \mathrm{mg} \text { were dissolved in } 10 \mathrm{~mL} \text { water and filtered sterile (100 } \\
\text { mM stock solution) }\end{array}$ \\
\hline IWP2 (5 mM) & $\begin{array}{l}10 \mathrm{mg} \text { dissolved in } 4.28 \mathrm{ml} \mathrm{DMSO} \text { and incubated at } 37^{\circ} \mathrm{C} \text { for } 10 \\
\mathrm{~min} \text {, stored at }-20^{\circ} \mathrm{C}\end{array}$ \\
\hline
\end{tabular}




\begin{tabular}{|l|l|}
\hline $\begin{array}{l}\text { Lactate/HEPES (1 } \\
\text { M stock solution) }\end{array}$ & $\begin{array}{l}3 \mathrm{ml} \text { of } 60 \% \text { sodium DL-lactate solution diluted in } 18 \mathrm{ml} \text { of } 1 \mathrm{M} \\
\text { HEPES sodium salt solution, stored at }-20^{\circ} \mathrm{C}\end{array}$ \\
\hline MTG (150 mM) & $\begin{array}{l}13 \mu \mathrm{l} \text { MTG diluted in } 1 \mathrm{ml} \text { IMDM and sterile filtered } \\
\text { freshly prepared before use }\end{array}$ \\
\hline TZV $(2 \mathrm{mM})$ & $10 \mathrm{mg}$ diluted in $6.8 \mathrm{ml} \mathrm{DMSO}$, stored at $-20^{\circ} \mathrm{C}$ \\
\hline
\end{tabular}

\subsection{Chemicals and solutions}

Table 9: Chemicals and solutions

\begin{tabular}{|l|l|}
\hline Component & Supplier, catalog number \\
\hline 2-Propanol & Merck Chemicals, 1096341000 \\
\hline Acetic acid (glacial) & Th. Geyer, \#2234-25L \\
\hline Agarose, peq Gold universal Agarose & Peqlab, \#732-2789 \\
\hline Alkaline Phosphatase staining kit & Sigma-Aldrich, \#86R-1KT \\
\hline B-Mercaptoethanol & Sigma-Aldrich, \#M3148 \\
\hline Bovine serum albumin (BSA), pH 7.0 & Sigma-Aldrich, \#A2153 \\
\hline $\begin{array}{l}\text { DAPI (4', 6-diamidino-2-phenylindole } \\
\text { dihydrochloride) }\end{array}$ & Sigma-Aldrich, \#D9542 \\
\hline Demecolcine (10 $\mu l / m l)$ & Sigma-Aldrich, \#D1925 \\
\hline dNTP Mix & Bioline, \#BIO-39029 \\
\hline DEPC-treated water & Ambion, \#AM9915G \\
\hline Ethanol 99\%, denatured & $\begin{array}{l}\text { WALTER CMP GmbH, \#WAL10503 } \\
1000\end{array}$ \\
\hline Ficoll-Paque Plus & GE-Healthcare \#17-1440-02 \\
\hline Fluo-4/AM, cell-permeant & Thermo Fisher Scientific \#F14201 \\
\hline Fluoromount-G & eBioscience \#00-4958-02 \\
\hline Formalin 37\% & Merck Millipore, \#1039991000 \\
\hline
\end{tabular}




\begin{tabular}{|c|c|}
\hline GeneAmp 10X PCR Buffer II \& $\mathrm{MgCl}_{2}$ & Thermo Fisher Scientific \#4379878 \\
\hline GeneRuler 100bp DNA ladder & Thermo Fisher Scientific, \#0321 \\
\hline Giemsa stain & Sigma-Aldrich, \#GS500 \\
\hline Glacial acetic acid & Merck Millipore, \#1.00063.1000 \\
\hline Glycerol & Roth \#3783 \\
\hline GoTaq G2 DNA Polymerase & Promega, \#M7841 \\
\hline Green GoTaq reaction buffer $5 x$ & Promega, \#M7911 \\
\hline HEPES & Roth, \#9105 \\
\hline HEPES sodium salt solution (1 M) & Sigma-Aldrich \#H3662 \\
\hline Hexadimethrine bromide (Polybrene) & Sigma Aldrich \#107689 \\
\hline Hydrochloric acid fuming $37 \%(\mathrm{HCl})$ & Merck Millipore \#100317 \\
\hline Loading buffer & Applichem, \#A3481 \\
\hline Magnesium chloride, $25 \mathrm{mM}$ & $\begin{array}{l}\text { Thermo Fisher Scientific, } \\
\text { \#N8080010 }\end{array}$ \\
\hline Methanol & Merck Millipore, $\# 106009$ \\
\hline Microscope slide & Nunc, \#177380 \\
\hline Microscope slide, SuperFrost Ultra Plus & Menzel, \#J3800AMNZ \\
\hline Midori Green Advance & Biozym, \#617004 \\
\hline MuLV Reverse Transcriptase $(50 \mathrm{U} / \mu \mathrm{l})$ & $\begin{array}{l}\text { Thermo Fisher Scientific, } \\
\text { \#N8080018 }\end{array}$ \\
\hline NHDF Kit & Lonza, VAPD-1001 \\
\hline Nuclease-free water & Thermo Fisher Scientific, \#AM9932 \\
\hline Oligo $d(T) 16$ Primer & $\begin{array}{l}\text { Thermo Fisher Scientific, } \\
\text { \#N8080128 }\end{array}$ \\
\hline Paraformaldehyde (PFA) & Sigma-Aldrich, \#158127 \\
\hline PCR buffer II 10x & $\begin{array}{l}\text { Thermo Fisher Scientific, } \\
\text { \#N8080010 }\end{array}$ \\
\hline Pluronic F-127 & Thermo Fisher Scientific \#P3000MP \\
\hline Potassium chloride $(\mathrm{KCl})$ & Sigma-Aldrich, \#P9541 \\
\hline
\end{tabular}




\begin{tabular}{|l|l|}
\hline QIAquick Gel Extraction kit & Qiagen, \#28706 \\
\hline RNase Inhibitor & $\begin{array}{l}\text { Thermo Fisher Scientific, } \\
\text { \#N8080199 }\end{array}$ \\
\hline Sodium chloride (NaCl) & Roth, \#9265.1 \\
\hline SV Total RNA isolation kit & Promega, \#Z3100 \\
\hline SYBR Green PCR master mix & Thermo Fisher Scientific, \#4309155 \\
\hline Tris & Roth, \#5429 \\
\hline Tris buffer pure & Roth, \#1022840 \\
\hline Triton X-100 & Sigma-Aldrich, \#3051.3 \\
\hline TrypLE Express Enzyme (1X), no phenol \\
red & $\begin{array}{l}\text { Thermo Fisher Scientific, } \\
\# 12604013\end{array}$ \\
\hline Tween 20 & Bio-Rad, \#170-6531 \\
\hline $\begin{array}{l}\text { VECTASHIELD Antifade Mounting } \\
\text { Medium }\end{array}$ & Vector Laboratories, \#H-1000 \\
\hline
\end{tabular}

\subsection{Devices and machines}

Table 10: Devices and machines

\begin{tabular}{|l|l|}
\hline Device & Company \\
\hline 7900 HT qPCR system & Thermo Fisher Scientific \\
\hline Balances: Extend & Sartorius \\
\hline Cell stimulator: MyoPacer ES & IonOptix \\
\hline $\begin{array}{l}\text { Centrifuges: 5810R, } \\
\text { 5415D, 5415R }\end{array}$ & Eppendorf \\
\hline $\begin{array}{l}\text { Chemiluminescence detection } \\
\text { system: ChemiDoc MP Imaging } \\
\text { System Universal Hood III }\end{array}$ & Bio-Rad \\
\hline
\end{tabular}




\begin{tabular}{|c|c|}
\hline $\begin{array}{l}\text { Confocal laser scanning microscope: } \\
\text { LSM } 710 \text { confocal microscopic } \\
\text { system }\end{array}$ & Carl Zeiss \\
\hline $\begin{array}{l}\text { Counting chamber: Neubauer } \\
\text { improved }\end{array}$ & Marienfeld Superior \\
\hline $\begin{array}{l}\text { DNA Isolation: DNA Maxwell } 16 \\
\text { System }\end{array}$ & Promega \\
\hline Flow cytometer: FACS Canto II & BD Biosciences \\
\hline Freezing box: Mr. Frosty & Thermo Fisher Scientific \\
\hline $\begin{array}{l}\text { Gel documentation: Multilmage } \\
\text { Light Cabinet }\end{array}$ & Alpha Innotech Corporation \\
\hline Incubator: Labotect C200 & Labotect \\
\hline $\begin{array}{l}\text { Microplate reader: Mithras LB 940, } \\
\text { Mithras LB } 943\end{array}$ & Berthold Technologies \\
\hline $\begin{array}{l}\text { Microscopes: Axio Observer A1, Axio } \\
\text { Observer Z1, Primo Vert, Axiovert } 25\end{array}$ & Carl Zeiss \\
\hline Multi-Electrode Array MEA 2100 & Multichannel Systems \\
\hline Multi-Image Light Cabinet & Alpha Innotech corporation \\
\hline $\begin{array}{l}\text { NanoDrop 2000c/2000 UV-Vis } \\
\text { Spectrophotometer }\end{array}$ & Thermo Fisher Scientific \\
\hline PCR cycler: Thermocycler 48 & SensoQuest \\
\hline pH meter: inoLab pH 7110 & WTW \\
\hline Pipet controller: Accu-jet pro & Brand \\
\hline $\begin{array}{l}\text { Pipettes: Reference, Research plus } \\
(10 / 100 / 1000 \mu \mathrm{L})\end{array}$ & Eppendorf \\
\hline $\begin{array}{l}\text { Power supply: Power } \\
\text { Pac } 3000-P o w e r \text { supply }\end{array}$ & Bio-Rad \\
\hline $\begin{array}{l}\text { qPCR cycler: iCycler Thermal Cycler, } \\
\text { iQ5 Multicolor Real-Time PCR } \\
\text { Detection System }\end{array}$ & Bio-Rad \\
\hline
\end{tabular}




\begin{tabular}{|l|l|}
\hline Sterile workbench: Hera Safe & Heraeus Instruments \\
\hline Tissue embedding & Leica Biosystems \\
system: Benchtop Tissue & \\
Processor 1020 & \\
\hline Water preparation system & Merck Millipore \\
Milli-Q Reference & \\
\hline
\end{tabular}

\subsection{Disposable items}

Table 11: Disposable items

\begin{tabular}{|c|c|}
\hline Item & Manufacturer \\
\hline 10-cm dish, TC-treated & CytoOne Starlab \#CC7682-3394 \\
\hline 12-well plate, TC-treated & CytoOne Starlab \#CC7682-7512 \\
\hline 6-cm dish, TC-treated & CytoOne Starlab \#CC7682-3359 \\
\hline 6-cm dish, untreated & Sarstedt \#82.1194.500 \\
\hline 6-well plate, TC- treated & CytoOne Starlab \#CC7682-7506 \\
\hline 96 -well microplate, black & Berthold Technologies \#23302 \\
\hline 96 -well microplate, black, clear bottom & Berthold Technologies \#38840 \\
\hline Cell scraper: 2-Posit. Blade 25 & Sarstedt \#83.1830 \\
\hline Cryo tubes & Thermo Fisher Scientific, \#377224 \\
\hline Filter tips: $0.1-1000 \mu \mathrm{L}$ & $\begin{array}{l}\text { Starlab \#S1120-3810, \#S1122-1830, } \\
\text { \#S1120-1840 }\end{array}$ \\
\hline $\begin{array}{l}\text { Flow cytometry tube: } 5 \mathrm{~mL} \text { Polystyrene } \\
\text { Round-Bottom Tubes }\end{array}$ & BD Falcon \#352058 \\
\hline Pasteur pipettes & Brand, \#747715 \\
\hline Pipette tips: $0.1-1000 \mu \mathrm{L}$ & $\begin{array}{l}\text { Starlab \#S1111-3700, \#S1111-1706, } \\
\text { \#S1112-1720 }\end{array}$ \\
\hline Pipettes: $5 \mathrm{~mL}, 10 \mathrm{~mL}, 25 \mathrm{~mL}$ & $\begin{array}{l}\text { Sarstedt \#86.1253.001, \#86.1254.001, } \\
\# 86.1685 .001\end{array}$ \\
\hline
\end{tabular}




\begin{tabular}{|l|l|}
\hline $\begin{array}{l}\text { Slides and coverslips: } 76 \times 26 \mathrm{~mm}, 18 \times \\
18 \mathrm{~mm} \text {, round } 25 \mathrm{~mm}\end{array}$ & $\begin{array}{l}\text { Thermo Fisher Scientific \#10143562CE, } \\
\text { Thermo Fisher Scientific \#4004672; R. } \\
\text { Langenbrinck, \#1049251 }\end{array}$ \\
\hline $\begin{array}{l}\text { Sterile filters: Millex-GS, } 0.22 \mu \mathrm{m} \text {; Steriflip } \\
50 \mathrm{~mL}, 0.22 \mu \mathrm{m} \text {; Steritops } 500 \mathrm{~mL}, 0.22 \\
\mu \mathrm{m}\end{array}$ & $\begin{array}{l}\text { Merck Millipore \#GLGS0250S } \\
\text { Merck Millipore \#SCGP00525 }\end{array}$ \\
\hline
\end{tabular}

\subsection{Software}

Table 12: Software

\begin{tabular}{|l|l|}
\hline Software & Company/Developer \\
\hline Adobe Illustrator 2020 & Adobe \\
\hline Adobe Photoshop & Adobe \\
\hline Adobe Reader & Adobe \\
\hline ApE A plasmid editor & M. Wayne Davis \\
\hline Axio Vision & Carl Zeiss \\
\hline Cardio 2D & Multichannel Systems \\
\hline Excel & Microsoft Corporation \\
\hline Flowing Software & Perttu Terho \\
\hline Graph Pad & GraphPad Software, Inc \\
\hline ImageJ / Fiji & Wayne Rasband, Curtis Rueden, and \\
& community \\
\hline Inkscape & Open-Source \\
\hline IQ optical systems software & Bio-Rad \\
\hline LabChart & AD Instruments \\
\hline MC_Rack & Multichannel Systems \\
\hline Multi Elec & (Georgiadis et al., 2015) \\
\hline SparkMaster & (Picht et al., 2007) \\
\hline Windows movie maker & Microsoft Corporation \\
\hline
\end{tabular}




\begin{tabular}{|l|l|}
\hline Word & Microsoft Corporation \\
\hline Zen & Carl Zeiss \\
\hline
\end{tabular}




\section{Methods}

\subsection{Cell culture techniques}

\subsubsection{Fibroblast isolation from skin punch biopsies}

Fibroblasts can be isolated from different tissues. One of the most accessible tissues is the skin, as fibroblasts can be readily cultivated from this tissue. Skin punch biopsies were obtained from probands and dissected mechanically with a scalpel and placed on $6 \mathrm{~cm}$ dishes in HFBM with an additional $10 \mathrm{ng} / \mathrm{ml} \mathrm{bFGF}$ and 2xP/S. Fibroblasts were allowed to grow out of the biopsy for three weeks in HFBM medium, which was changed every other day. For passaging, cells were incubated in $0.25 \%$ Trypsin/EDTA until they started to detach. Trypsin/EDTA was removed and cells were rinsed and collected with HFBM and transferred to a new dish. Consequently, fibroblasts were cultivated in HFBM and used for iPSC-production at passages 2-7.

\subsubsection{PBMC isolation}

Human PBMCs were isolated from donor blood samples. Fifteen $\mathrm{ml}$ of blood were drawn in an EDTA-vacutainer and PBMCs were isolated. The blood was transferred to a $50 \mathrm{ml}$ falcon tube and $35 \mathrm{ml}$ DMEM/F12 was added. Two times $25 \mathrm{ml}$ of the solution was added to two $50 \mathrm{ml}$ falcons containing $20 \mathrm{ml}$ Ficoll-Paque. Falcons were centrifuged at $400 \mathrm{~g}$ for $40 \mathrm{~min}$ at room temperature without deceleration break. hPBMCs were collected from the interphase in a $50 \mathrm{~mL}$ falcon tube and DMEM/F12 was added until $50 \mathrm{~mL}$ total volume was reached. Cells were centrifuged at $750 \mathrm{~g}$ for $10 \mathrm{~min}$ at room temperature and supernatant was discarded. Cells were resuspended in $40 \mathrm{ml}$ DMEM/F12 and centrifuged at $750 \mathrm{~g}$ for $10 \mathrm{~min}$ at room temperature and supernatant was discarded. Cells were resuspended in 1-2 $\mathrm{ml}$ blood medium and counted. In total, $2 \times 10^{6}$ cells were added in one well of a 24-well plate in blood medium with $1 \times \mathrm{P} / \mathrm{S}$. Medium was changed every other day by collecting the medium and subsequent centrifugation at $300 \mathrm{~g}$ for $5 \mathrm{~min}$. 
Supernatant was discarded and cells resuspended in new blood medium and added to the old well.

\subsubsection{IPSC reprogramming of fibroblasts and PBMCS}

Fibroblasts were reprogrammed at passages 2-7 and PBMCs after 5-20 days post isolation. For reprogramming, a plasmid-based non-integrating approach was used for fibroblasts and a Sendai virus-based non-integrative approach was used for fibroblast and PBMCs.

\subsubsection{Plasmid reprogramming}

For rapid and cheap reprogramming the use of plasmids is useful as demonstrated by Okita et al. (Okita et al., 2011). Fibroblasts were washed once with PBS and digested with $0.25 \%$ Trypsin/EDTA or TrypLE for $1-2$ min at $37^{\circ} \mathrm{C}$ to obtain single cells. Cells were collected in fibroblast medium and counted. $5 \times 10^{5}-1 \times 10^{6}$ cells were transferred to an Eppendorf cup and centrifuged for $10 \mathrm{~min}$ at $200 \mathrm{~g}$. Nucleofector solution $(82 \mu \mathrm{l})$ and supplement $(18 \mu \mathrm{l})$ were mixed and the cells were resuspended in the mix. 1-2 $\mu \mathrm{g}$ of each of the plasmids was added and the solution was mixed properly. Cells were transferred to the supplied cuvettes and nucleofection was carried out in the Amaxa nucleofector II with program P22 or U23. Cells were carefully resuspended in HFBM and transferred to a prepared plate with HFBM with 2xP/S and $2 \mu \mathrm{M} \mathrm{TZV}$. The cells were allowed to grow for one week with medium change with HFBM supplemented with $0.5 \mathrm{mM}$ sodium butyrate every other day. Afterwards, cells were split with $0.25 \%$ Trypsin/EDTA or TrypLE and diluted onto new geltrex coated 6-well plates. From day 8 on, cells were supplemented with essential 8 (E8) medium. From day 8 to day 11, E8 medium was supplemented with $0.5 \mathrm{mM}$ sodium butyrate to enhance reprogramming. After 30 days iPSC colonies were mechanically collected in geltrex coated 12-well plates in E8 medium with $2 \mu \mathrm{M} \mathrm{TZV}$. 


\subsubsection{Sendai reprogramming}

Sendai viruses are integration-free RNA viruses and therefore especially useful for iPSC production. For reprograming, the cyto tune 2.0 Sendai virus kit from life technologies was used. In brief, $3 \times 10^{5}-5 \times 10^{5}$ fibroblasts or PBMCs were used for transduction. Medium was removed and Sendai viruses were added to the cells in fresh HFBM or blood medium with $1 \mathrm{xP} / \mathrm{S}$ at a MOI of 2.5/2.5/1.5, 5/5/3, or 10/10/5 for the Sendai viruses hKOS, hc-Myc, or h-Klf4, respectively. Virus solution volumes were calculated from the virus concentration stated on the analysis certificate and the desired MOI (multiplicity of infection). The cells were incubated with the virus for $24 \mathrm{~h}$ and supplemented with fresh HFBM/blood medium. After seven days, the fibroblasts and PBMCs were transferred to a geltrex well of a 6-well plate, which was coated with geltrex, at a 1:2-1:24 rate and medium was changed to E8 medium. Four to five weeks after transduction, emerging colonies were manually picked onto geltrex-coated 12-well plates in E8 medium.

\subsubsection{Cultivation of iPSCs}

During this work, iPSCs were either cultured on mouse embryonic fibroblasts (MEF) or feeder-free cultivation methods with geltrex were used. For geltrex coating, $2 \mathrm{mg}$ of -20 ${ }^{\circ} \mathrm{C}$ cold geltrex was dissolved in $12 \mathrm{ml}$ of DMEM/F12. Twelve-well plates were coated with $0.5 \mathrm{~mL}$ geltrex solution and 6-well plates with $1 \mathrm{ml}$ per well.

For feeder-free culture, IPSCs were cultivated on geltrex-coated 6-well plates and supplemented with E8 medium. Medium was changed every day. The cells were split twice per week. In brief, cells were washed with versene followed by 4 min of incubation with versene at room temperature. Afterwards, versene was discarded and the cells were collected in E8 medium supplemented with $2 \mu \mathrm{M}$ TZV and a fraction (1:1-1:30) of them was transferred to new geltrex coated 6-well plates. Medium was changed to normal E8 the next day.

For culture on MEFs, iPSCs were incubated in hESC-medium and medium was changed every two days. During passaging, cells were washed once with DMEM/F12 followed by 
incubation with collagenase IV $(200 \mathrm{U} / \mathrm{ml})$ for $5 \mathrm{~min}$ at $37^{\circ} \mathrm{C}$. Cells were washed two times with DMEM/F12 and a cell scraper was used to detach them. Cells were resuspended in hESC-medium using a $1 \mathrm{ml}$ pipette and transferred to new $6 \mathrm{~cm}$ plates with MEF.

\subsubsection{Freezing and thawing of hiPSCs}

For feeder-free culture, one confluent well of a 6-well plate with hiPSCs was detached as described earlier (3.1.6) in $1 \mathrm{ml}$ E8 medium and collected in a cryovial. Subsequently, 1 $\mathrm{ml}$ double concentrated freezing medium was added dropwise. The cryovial was transferred to a $-80{ }^{\circ} \mathrm{C}$ freezer in a Mister Frosty box to guarantee a slow temperature decrease. Frozen cells were transferred to long-term liquid nitrogen storage on the next day.

IPSCs cultivated on MEFs were detached as described before. Cells were resuspended in DMEM/F12. The cells were centrifuged for 3 min at $200 \mathrm{~g}$. DMEM/F12 was discarded and the pellet was resuspended in freezing medium. The cells were transferred to cryovials and frozen in a Mister Frosty box at $-80^{\circ} \mathrm{C}$. The next day, they were transferred to liquid nitrogen storage.

To thaw iPSCs, cryovials were removed from liquid nitrogen storage warmed briefly in a $37^{\circ} \mathrm{C}$ water bath. Afterwards, the cell suspension was transferred to a $15 \mathrm{ml}$ falcon tube and subsequently $10 \mathrm{ml}$ of DMEM/F12 was added. Cells were centrifuged for $3 \mathrm{~min}$ at 200 g, supernatant was removed and the cells resuspended in hES-Medium or E8-medium (with $2 \mu \mathrm{M} \mathrm{TZV}$ ) and seeded onto $6 \mathrm{~cm}$ dishes (feeder layer culture) or geltrex coated 6well plates (feeder-free culture).

\subsubsection{Embryoid body differentiation}

To test the in vitro differentiation potential of iPSCs, untargeted random differentiation via EBs was used. At day 0, iPSCs cultivated on MEFs were collected for comparison: Medium was discarded and cells were washed with PBS. Subsequently, cells were 
collected in PBS with a cell scraper and centrifuged at $17,000 \mathrm{~g}$ for $1 \mathrm{~min}$ and the supernatant was discarded. Pellets were snap-frozen in liquid nitrogen and stored at $80^{\circ} \mathrm{C}$ until usage. For EB differentiation, iPSCs cultivated on MEFs were washed with $\mathrm{DMEM} / \mathrm{F} 12$. The cells were digested with collagenase IV for up to $5 \mathrm{~min}$ and washed again with DMEM/F12. The culture was sliced in medium chunks with the help of a cell scraper, collected and transferred to an uncoated $6 \mathrm{~cm}$ dish. After one day, the medium was changed to Iscove medium by collecting cells in a $15 \mathrm{ml}$ falcon tube and carefully aspirating the supernatant followed by resuspension in Iscove medium. For the first seven days, the cells were cultivated in suspension culture and the medium was changed every other day. On day 8, a part of the culture was collected in form of a pellet by washing the EBs once with PBS and centrifugation of the EBs at $17,000 \mathrm{~g}$ for $1 \mathrm{~min}$. The supernatant was discarded and cell pellets were snap-frozen in liquid nitrogen and stored at $-80^{\circ} \mathrm{C}$ until usage. The rest of the EBs was transferred to a $0.1 \%$ gelatin-coated tissue culture treated $6 \mathrm{~cm}$ dish. Iscove medium was changed every other day. On day $8+5$, one dish of EBs was fixed for immunostaining as described in chapter 3.2.12. On day $8+25$, pellets were collected and the rest of the EB dishes were fixed for immunostaining as described in chapter 3.2.12. The pellets were used for RNA isolation and reverse transcription PCR (RT-PCR) and PCR for differentiation-related genes. The fixed bodies were used for immunostaining for $\beta$-tubulin, $\alpha$-smooth muscle actin ( $\alpha-S M A)$, and alpha-fetoprotein (AFP).

\subsubsection{In vivo differentiation of iPSCs in SCID mice}

One traditional method to check the pluripotency of a cell is a teratoma assay. In that assay, cells are injected in immunosuppressed mice, as they proliferate, subsequently form tissue of all three germ layers. The experiments were performed in cooperation with Prof. Ralf Dressel (Department of Immunology, UMG) and hematoxylin-eosin staining was performed by the Department of Pathology (UMG).

Briefly, two to four $6 \mathrm{~cm}$ dishes of iPSCs on MEF were washed with DMEM/F12 followed by incubation with collagenase IV for $4 \mathrm{~min}$ at $37^{\circ} \mathrm{C}$. Cells were washed with DMEM/F12 
and scraped with a cell scraper and collected in $2 \mathrm{mI} D M E M / F 12$. Cells from $3 \times 6 \mathrm{~cm}$ plates were pooled and centrifuged at $200 \mathrm{~g}$ for $2 \mathrm{~min}$ and the supernatant was discarded. Cells were resuspended in $200 \mu \mathrm{l}$ DMEM/F12 before being injected into SCID mice. After several months and detection of palpable teratomas, mice were sacrificed and teratomas were explanted. Teratomas were fixed in $37 \%$ formalin, cut into thin slices, and stained with hematoxylin and eosin. Stained teratomas were assessed for the occurrence of tissues of different germ layers by light microscopy.

\subsubsection{Directed in vitro iPSC-differentiation, cardiomyocyte culturing, metabolic selection and digestion}

IPSCs cultured feeder-free on geltrex were used for cardiac differentiation. Cardiac differentiation of iPSCs was performed by sequential manipulation of the WNT pathway and is depicted schematically in Figure 4 . At $85-95 \%$ cell confluence, media was changed to cardio differentiation medium supplemented with $4 \mu \mathrm{M}$ of the glycogen synthase kinase 3 (GSK3) inhibitor CHIR. After $48 \mathrm{~h}$, medium was changed to fresh cardio differentiation medium with addition of $5 \mu \mathrm{M}$ of the inhibitor of IWP2 for two days. On day 4 , medium was changed to plain cardio differentiation medium. From day 10 on, the cells were cultured in cardio culture medium. Medium was changed every two to three days. To purify iPSCCMs after 20-40 days of differentiation, metabolic selection was carried out by culturing the cells for 2-4 days in cardio-selection medium containing lactate as carbon source. Differentiated iPSC-CMs were studied on day $60+$ after initiation of differentiation. Following differentiation, purity of iPSC-CMs was determined with the help of flow cytometry analysis. For experiments, only iPSC-CMs with purities of above $75 \% \mathrm{cTnT}$ positive cells were used.

For immunofluorescence and MEA experiments, iPSC-CMs have to be digested and transferred to the respective experimental surface. Cells were incubated in $0.25 \%$ Trypsin/EDTA at $37{ }^{\circ} \mathrm{C}$ for $5 \mathrm{~min}$. Cells were detached and collected with cardio digest medium and transferred to a $15 \mathrm{ml}$ falcon tube. After centrifugation for $5 \mathrm{~min}$ at $200 \mathrm{~g}$, supernatant was removed, cells were resuspended in cardio digest medium and counted. 
Subsequently, cells were transferred to geltrex coated MEAs or coverslips (for IF). After 1-day, medium was changed to cardio culture medium.

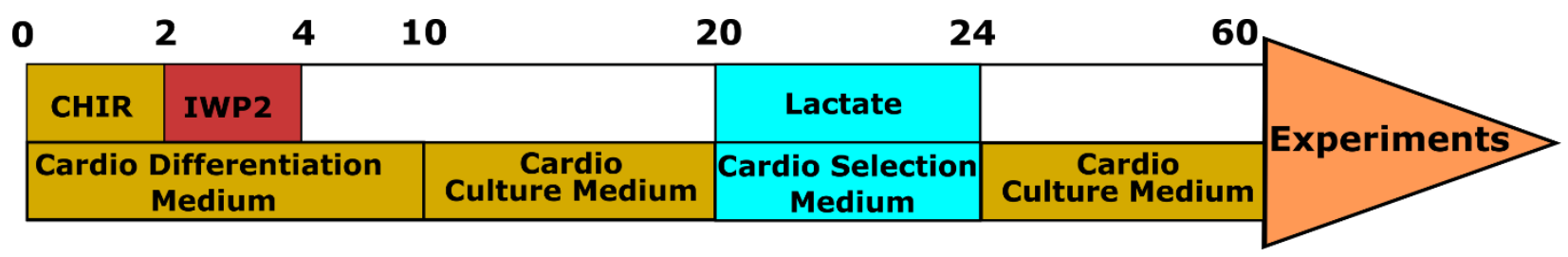

Figure 4: Schematic view of the differentiation protocol used in this study. Cardiac induction was performed by CHIR and IWP2 addition followed by 16 days of differentiation. Metabolic selection was performed using lactate. Afterwards, cells were allowed to mature until day 60 .

\subsubsection{Iso/Epi treatment}

Cardiomyocytes were stressed by the addition of the catecholamines Iso and Epi to achieve a TTS-like phenotype. $247.7 \mathrm{mg}$ of Iso were dissolved in $10 \mathrm{ml}$ water and filtered sterile to obtain $100 \mathrm{mM}$ stock solution. $109.8 \mathrm{mg}$ of Epi were dissolved in $10 \mathrm{ml}$ water and filtered sterile to obtain a $50 \mathrm{mM}$ stock solution. Iso and Epi stock solutions were prediluted in sterile water at $100 \mathrm{x}$ the desired concentrations and stored at $-20{ }^{\circ} \mathrm{C}$. For experiments, 1:100 dilution from the $100 x$ stock was added to the cells to achieve catecholamine concentrations of $100 \mathrm{nM}$ (Epi/lso), $1 \mu \mathrm{M}$ (Epi/lso), $10 \mu \mathrm{M}$ (Epi/lso), $100 \mu \mathrm{M}$ (Epi/lso), $500 \mu \mathrm{M}$ (Epi only), $1 \mathrm{mM}$ (Iso only), $5 \mathrm{mM}$ (Iso only). The cardiomyocytes were incubated with the catecholamines for $2 \mathrm{~h}$ if not stated otherwise.

\subsubsection{Pellet collection}

IPSC and iPSC-CM pellets had to be collected fast and reliable in order to analyze their RNA and protein content. Medium was discarded and cells were washed once with PBS. Subsequently, cells were collected in PBS with a cell scraper and centrifuged at 17,000 g for $1 \mathrm{~min}$. The supernatant was discarded and pellets were snap-frozen in liquid nitrogen and stored in a $-80^{\circ} \mathrm{C}$ freezer until usage. 


\subsection{Molecular biology}

\subsubsection{RNA isolation}

To analyze expression of genes, the RNA was analyzed. To isolate RNA, the SV total RNA isolation kit from Promega was used. The cell pellets were resuspended in $400 \mu \mathrm{l}$ of lysis buffer, transferred to a new Eppendorf cup and mixed with $400 \mu \mathrm{l}$ of $95 \%$ ethanol. Solution was transferred to a spin column and centrifuged for 1 min at $13,000 \mathrm{~g}$. The column was washed with $600 \mu \mathrm{l}$ RNA wash solution. The column was centrifuged at $13,000 \mathrm{~g}$ for $1 \mathrm{~min}$. DNAse solution was added to the column and incubated for 15 minutes at room temperature. To stop the reaction, DNase stop solution (200 $\mu$ l) was added and the column was centrifuged at 13,000 $\mathrm{g}$ for $1 \mathrm{~min} .600 \mu \mathrm{l}$ RNA wash solution was added and the column was centrifuged at $13,000 \mathrm{~g}$ for $1 \mathrm{~min}$. $250 \mu \mathrm{l}$ of RNA wash solution were added to the column and the column was centrifuged for $2 \mathrm{~min}$ at $13,000 \mathrm{~g}$ and one additional time at $13,000 \mathrm{~g}$ for $1 \mathrm{~min}$. RNA elution was carried out by adding $100 \mu \mathrm{l}$ of nuclease-free water to the column followed by a 1 min centrifugation at $13,000 \mathrm{~g}$. RNA concentration was measured at $260 / 280 \mathrm{~nm}$ with a spectrometer or with a Nanodrop.

\subsubsection{Reverse transcription PCR}

To transcribe the isolated RNA into complementary DNA (cDNA) reverse transcription PCR (RT-PCR) was used with the help of reverse transcriptase.

The following reaction was prepared:

Table 13: Components for RT-PCR

\begin{tabular}{|l|l|}
\hline RNA & $\mathbf{1 0 0} \mathbf{~ n g}$ \\
\hline $10 x$ PCR buffer II & $2 \mu \mathrm{l}$ \\
\hline $25 \mathrm{mM} \mathrm{MgCl}_{2}$ & $4 \mu \mathrm{l}$ \\
\hline $100 \mathrm{mM}$ dNTPs & $0.8 \mu \mathrm{l}$ \\
\hline RNase Inhibitor $(20 \mathrm{U} / \mu \mathrm{l})$ & $1 \mu \mathrm{l}$ \\
\hline
\end{tabular}




\begin{tabular}{|l|l|}
\hline $50 \mu \mathrm{M}$ Oligo $(\mathrm{dt})_{16}$ & $1 \mu \mathrm{l}$ \\
\hline MuLV Reverse Transcriptase $(50 \mathrm{U} / \mu \mathrm{L})$ & $1 \mu \mathrm{l}$ \\
\hline Nuclease free water & $\mathrm{x} \mu \mathrm{l}$ \\
\hline final volume & $20 \mu \mathrm{l}$ \\
\hline
\end{tabular}

The reaction was incubated as follows:

Table 14: Temperature for RT-PCR

\begin{tabular}{|l|l|}
\hline Temperature & time \\
\hline $22{ }^{\circ} \mathrm{C}$ & $10 \mathrm{~min}$ \\
\hline $42{ }^{\circ} \mathrm{C}$ & $50 \mathrm{~min}$ \\
\hline $95^{\circ} \mathrm{C}$ & $10 \mathrm{~min}$ \\
\hline $4{ }^{\circ} \mathrm{C}$ & $\infty$ \\
\hline
\end{tabular}

\subsubsection{Polymerase chain reaction (PCR)}

To analyze expression levels of cDNA it is necessary to amplify it. This is done by PCR. One to two $\mu \mathrm{l}$ of cDNA reaction were added as PCR template to the reagents from Table 15. When using two $\mu$ of cDNA nuclease-free water volume from Table 15 was reduced to $14.05 \mu$ l. The PCR was run for 25-35 cycles according to temperatures and incubation times from Table 16.

Table 15: Components for PCR

\begin{tabular}{|l|l|}
\hline Components for PCR & Volume $(\boldsymbol{\mu l})$ \\
\hline cDNA & 1 \\
\hline Nuclease free water & 15.05 \\
\hline $5 x$ Green GoTaq reaction buffer & 5 \\
\hline $10 \mathrm{mM}$ dNTPs & 1.6 \\
\hline Forward primer $(10 \mu \mathrm{M})$ & 1 \\
\hline Reverse primer $(10 \mu \mathrm{M})$ & 1 \\
\hline
\end{tabular}




\begin{tabular}{|l|l|}
\hline GoTaq DNA polymerase & 0.1 \\
\hline DMSO & 0.25 \\
\hline Overall volume & 25 \\
\hline
\end{tabular}

Table 16: Conditions for PCR

\begin{tabular}{|l|l|l|}
\hline Temperature & Duration & Cycle number \\
\hline $95^{\circ} \mathrm{C}$ & $120 \mathrm{~s}$ & $1 \mathrm{x}$ \\
\hline $95^{\circ} \mathrm{C}$ & $15 \mathrm{~s}$ & $\mathrm{nx}$ \\
\hline $51-66^{\circ} \mathrm{C}$ & $10 \mathrm{~s}$ & \\
\hline $72{ }^{\circ} \mathrm{C}$ & $20 \mathrm{~s}$ & $1 \mathrm{x}$ \\
\hline $95^{\circ} \mathrm{C}$ & $15 \mathrm{~s}$ & $72 \mathrm{x}$ \\
\hline $60^{\circ} \mathrm{C}$ & $10 \mathrm{~s}$ & \\
\hline $4{ }^{\circ} \mathrm{C}$ & $\infty$ & \\
\hline
\end{tabular}

GAPDH was used as control. Table 2 contains primer sequences, cycle number $(n)$ and annealing temperatures used for PCR analyses. For visualization, 1-2\% agarose gels with $6 \mu \mathrm{l}$ Midori green was used. Gels were loaded with $15 \mu \mathrm{l} \mathrm{PCR}$ products (already containing loading buffer from the $5 x$ Green GoTaq reaction buffer) and run at $100 \mathrm{~V}$ for 30-45 min. $7 \mu$ of the Gene Ruler 100 bp DNA ladder was used for size comparison. Visualization and image acquisition were carried out in a Multilmage Light Cabinet.

\subsubsection{Quantitative PCR}

Quantitative PCR (qPCR) was used to quantify gene expression on mRNA level. For this purpose, fluorescent SYBR green was used to quantify cDNA in the setting of a PCR. To perform qPCR, the following amounts were used in a 96-well or 384-well format:

Table 17: Setup for qPCR 


\begin{tabular}{|l|l|}
\hline $10 \mu \mathrm{l}$ SYBR Green & $5 \mu \mathrm{l}$ Sybr Green \\
\hline $7 \mu \mathrm{l}$ nuclease-free water & $3.5 \mu \mathrm{l}$ nuclease-free water \\
\hline $1 \mu \mathrm{l}$ primer forward & $0.25 \mu \mathrm{l}$ primer forward \\
\hline $1 \mu \mathrm{l}$ primer reverse & $0.25 \mu \mathrm{l}$ primer reverse \\
\hline $1 \mu \mathrm{l} \mathrm{cDNA}$ sample & $1 \mu \mathrm{l}$ cDNA sample \\
\hline
\end{tabular}

The following temperature settings were used for 96-well plates in a BioRad qPCR system.

Table 18: Temperature, duration, and cycle setup for the BioRad qPCR system

\begin{tabular}{|l|l|l|}
\hline Temperature & Duration & Cycle number \\
\hline $95^{\circ} \mathrm{C}$ & $120 \mathrm{~s}$ & $1 \mathrm{x}$ \\
\hline $95^{\circ} \mathrm{C}$ & $15 \mathrm{~s}$ & \multirow{2}{*}{$40 \mathrm{x}$} \\
\hline $60^{\circ} \mathrm{C}$ & $10 \mathrm{~s}$ & \\
\hline $72{ }^{\circ} \mathrm{C}$ & $20 \mathrm{~s}$ & $1 \mathrm{x}$ \\
\hline $95^{\circ} \mathrm{C}$ & $15 \mathrm{~s}$ & $72 \mathrm{x}$ \\
\hline $60^{\circ} \mathrm{C}$ & $10 \mathrm{~s}$ & \\
\hline $4{ }^{\circ} \mathrm{C}$ & $\infty$ & \\
\hline
\end{tabular}

Temperature settings in 384-well plates in the $7900 \mathrm{HT}$ system were as follows.

Table 19: Temperature, duration, and cycle setup for the 7900 HT qPCR system

\begin{tabular}{|l|l|l|}
\hline Temperature & Duration & Cycle number \\
\hline $50^{\circ} \mathrm{C}$ & $120 \mathrm{~s}$ & $1 \mathrm{x}$ \\
\hline $95^{\circ} \mathrm{C}$ & $600 \mathrm{~s}$ & $1 \mathrm{x}$ \\
\hline $95^{\circ} \mathrm{C}$ & $15 \mathrm{~s}$ & $40 \mathrm{x}$ \\
\hline $60^{\circ} \mathrm{C}$ & $60 \mathrm{~s}$ & \\
\hline $95^{\circ} \mathrm{C}$ & $15 \mathrm{~s}$ & $1 \mathrm{x}$ \\
\hline
\end{tabular}




\begin{tabular}{|l|l|l|}
\hline $60^{\circ} \mathrm{C}$ & $15 \mathrm{~s}$ & $72 \mathrm{x}$ \\
\hline $95^{\circ} \mathrm{C}$ & $15 \mathrm{~s}$ & $1 \mathrm{x}$ \\
\hline
\end{tabular}

GAPDH was used as a control and the qPCR was analyzed and normalized to it via the delta $C_{t}$ method. In brief, $C_{t}$ values of GAPDH were subtracted from the $C_{t}$ values of the gene of interest of the same sample.

\subsubsection{DNA isolation and sequencing}

For genetic analysis, isolation of genomic DNA was necessary. The Maxwell 16 DNA purification kit (Promega) was used in combination with the automatic Maxwell 16 instrument. Cell pellets were resuspended in $350 \mu \mathrm{l}$ PBS and placed in a provided cartridge. Three-hundred $\mu \mathrm{l}$ of the elution buffer were added to the elution tube. A plunger was loaded into its respective well, the cartridge was placed in the Maxwell 16 instrument and the program "Cells" was carried out. Afterwards, the remaining magnetic beats were removed with the help of a magnetic rack. The eluate was collected and DNA concentrations were measured at $260 / 280 \mathrm{~nm}$ in a photo spectrometer.

One-hundred ng of the isolated DNA were used as PCR template and the region of interest was amplified by PCR as described in chapter 3.2.3. The product was run on a $1.5 \%$ agarose gel as described in chapter 3.2.3 and the desired DNA fragment was cut out with a scalpel. The product was purified with QIAquick Gel Extraction Kit (Qiagen). The gel fragment was weighted to estimate the volume of the fragment. One volume of gel fragment was mixed with 3 volumes of $Q G$ buffer and one volume of isopropanol. The solution was transferred to a QIAquick column and centrifuged at $13,000 \mathrm{~g}$ for $1 \mathrm{~min}$. The flow-through was discarded. Afterwards, the column was washed by addition of $750 \mu \mathrm{l}$ of PE buffer followed by another 1 min centrifugation step at 13,000 g. The flowthrough was discarded and the centrifugation was repeated. The column was placed in a $1.5 \mathrm{ml}$ eppendorf cup and the DNA was eluted by adding $50 \mu \mathrm{l}$ of nuclease-free water and centrifugation was carried out at $13,000 \mathrm{~g}$ for $1 \mathrm{~min}$. DNA concentrations were measured by a photo spectrometer at $260 / 280 \mathrm{~nm}$. To prepare the sequencing reaction $10 \mathrm{pmol}$ 
sequencing primer and $67.5 \mathrm{ng}$ DNA were mixed in $15 \mu \mathrm{l}$ nuclease-free water. The sequencing itself was done externally by Seqlab Sequencing Laboratories $\mathrm{GmbH}$. Results were analyzed with $\mathrm{ApE}$.

\subsubsection{Alkaline phosphatase (ALP) staining}

Pluripotent stem cells express ALP. The enzyme can be visualized by using its catalytic activity. To stain for ALP, a staining kit (Sigma-Aldrich, \#86R-1KT) was used. Fixation solution was prepared as follows: $13 \mathrm{ml}$ acetone, $5 \mathrm{ml}$ citrate solution, and $1.6 \mathrm{ml} 37 \%$ formaldehyde were mixed in a glass bottle. Cells were washed two times with PBS. Fixation solution was added and dishes were incubated for 30 seconds. Cells were washed twice with water. Staining solution was prepared as follows: $0.5 \mathrm{ml} \mathrm{FRV-alkaline}$ solution was mixed with $0.5 \mathrm{ml}$ sodium nitrate and incubated for 2 min at room temperature. Then, $22.5 \mathrm{ml}$ water and $0.5 \mathrm{ml}$ Naphthol AS-B were added. The staining solution was added and incubated for at least $15 \mathrm{~min}$ at $37^{\circ} \mathrm{C}$ in the dark. The cells were washed two times with water and air-dried overnight.

\subsubsection{Annexin V/PI staining}

Apoptosis is the process of regulated cell death and often occurs under intensive stress conditions. To quantify the rate of apoptosis in our system, a combination of annexin $\mathrm{V}$ and propidium iodide $(\mathrm{PI})$ staining was used. It is a hallmark of annexin $\mathrm{V}$ that it is normally located in the inner cell membrane. Only if apoptosis starts, the cell membrane starts to lose its normal organization and annexin $\mathrm{V}$ can be stained with extracellular staining at the outer cell membrane. PI is a DNA staining agent and stains late apoptotic or necrotic cells, with DNA being abundant all over the cell due to membrane damage.

The cells were digested with $0.25 \%$ Trypsin/EDTA, collected in FBS, and counted. After a centrifugation step at $200 \mathrm{~g}$ for $3 \mathrm{~min}, 1 \times 10^{6} \mathrm{cells} / \mathrm{ml}$ were resuspended in PBS. Cells were centrifuged at $200 \mathrm{~g}$ for $3 \mathrm{~min}$ and subsequently resuspended in $500 \mu \mathrm{l}$ annexin $\mathrm{V}$ binding buffer. $100 \mu \mathrm{l}$ of the cell solution was stained with one of the following stainings: 
No staining

$5 \mu$ Innexin V

$10 \mu \mathrm{I}$ P solution

$5 \mu \mathrm{l}$ annexin $\mathrm{V}$ and $10 \mu \mathrm{PI}$ solution

The stainings were incubated at room temperature for $15 \mathrm{~min}$ in the dark. $400 \mu \mathrm{l}$ annexin $v$ binding buffer was added and 10,000 cells were analyzed with a flow cytometer and recorded with the FACSDiva software. Analysis for the fraction of annexin v positive and annexin v/PI (necrotic) positive cells was performed with flowing software.

\subsubsection{Analysis of sarcomeric regularity}

To analyze sarcomeric regularity, iPSC-CMs were stained for a-actinin, as described previously. FIJI with the integrated plugin "Tubeness" was used on the obtained images. Next, the fast Fourier transform algorithm was used on the data. The resulting frequency domains were radially integrated with the help of the open-source plugin "Radial Profile Plot" from Paul Baggethun. Finally, analysis of the relative amplitude of the first peak of the intensity profile was performed with LabChart. This extracted amplitude level was used to analyze the regularity of sarcomeres in the iPSC-CMs.

\subsection{9 cTnT flow cytometry analysis}

To quantify the efficiency of the cardiac differentiation, flow cytometry for the cardiac marker cTNT was performed. Briefly, cardiomyocytes were digested for 10 min with $0.25 \%$ Trypsin/EDTA to obtain single cells. Digestion was stopped with cardio culture medium with 20\% FBS and cells were counted. 250,000 cells per tube were collected and centrifuged for $5 \mathrm{~min}$ at $200 \mathrm{~g}$. Cells were washed once with PBS. Fixation was performed in $4 \%$ PFA for 20 min at room temperature. After fixation, cells were washed twice with PBS. For permeabilization, cells were incubated for $15 \mathrm{~min}$ in $0.1 \%$ Triton-X 100/1\% BSA 
in PBS. Cells were incubated with the primary antibody (cTNT 1:500 in 0.1\% Triton-X $100 / 1 \%$ BSA) overnight at $4{ }^{\circ} \mathrm{C}$. Cells were washed two times with Triton-X 100/1\% BSA in PBS and the second antibody (Alexa 488) was added in $0.1 \%$ Triton-X 100/1\% BSA. The secondary antibody was incubated for $45 \mathrm{~min}$ at $37^{\circ} \mathrm{C}$ in the dark. Cells were washed two times with $0.2 \%$ BSA. Cells were resuspended in PBS and 10,000 cells were analyzed using a flow cytometer with the FACS Diva software. Unstained cells were used as negative control and cells stained only with the secondary antibody were used to perform gating settings. Analysis was performed with the FACS Diva software and flowing software.

\subsubsection{0 $\mathrm{Ca}^{2+}$ imaging}

Cardiomyocyte contraction relies on cyclic $\mathrm{Ca}^{2+}$ changes in the cells, which are characterized by $\mathrm{Ca}^{2+}$ transients and $\mathrm{Ca}^{2+}$ sparks. For $\mathrm{Ca}^{2+}$ imaging iPSC-CMs were cultivated on round coverslips coated with geltrex. To visualize these $\mathrm{Ca}^{2+}$ fluctuations, fluo-4 based $\mathrm{Ca}^{2+}$ imaging is used.

Cells were imaged in Tyrode solution:

$\begin{array}{ll}\mathrm{NaCl} & 140 \mathrm{mM} \\ \mathrm{KCl} & 5.4 \mathrm{mM} \\ \mathrm{MgCl}_{2} & 1 \mathrm{mM} \\ \text { HEPES } & 10 \mathrm{mM} \\ \text { Glucose } & 10 \mathrm{mM} \\ \mathrm{CaCl}_{2} & 1.8 \mathrm{mM}\end{array}$

$50 \mu \mathrm{g}$ Fluo4 were solved in $44 \mu \mathrm{l}$ DMSO to obtain a stock solution. Two $\mu \mathrm{l}$ of the stock solution were mixed with $0.5 \mu \mathrm{l}$ pluronic and mixed with $400 \mu \mathrm{l}$ Tyrode to obtain a staining solution. Coverslips with iPSC-CMs were attached to the $\mathrm{Ca}^{2+}$ measuring chamber and stained for 25-30 min with fresh staining solution with or without CGP (100 nM), ICI (50 
$\mathrm{nM}$ ), or both. After staining, cells were washed twice with Tyrode and measured in Tyrode. After measuring basal conditions, fresh Tyrode with increasing Iso concentrations and CGP/ICI depending on the conditions was added.

Cells were measured with a Carl Zeiss LSM 710 confocal microscope and a $63 \times 1.4$ NA oil objective in line scan mode (512 pixels, $45.5 \mu \mathrm{m}, 1057.7 \mathrm{~Hz}, 20,000$ cycles). For Fluo4 excitation was done at $488 \mathrm{~nm}$ and emission was detected at $490-540 \mathrm{~nm}$. The pinhole was set to 6 airy units.

The analysis was performed with GraphPad, Excel, Fiji, and LabChart. For Line scan intensity export, Fiji was used. Exported data were smoothed with GraphPad and consequently analyzed with LabChart. $T_{50}$ was defined as the time from peak fluorescence until $50 \%$ signal decay. The rise time was defined as the time from transient start to the time of maximum level of the transient.

\subsubsection{1 $\mathrm{Ca}^{2+}$ spark analysis}

Cardiomyocytes typically show $\mathrm{Ca}^{2+}$ sparks, which are small spatially defined $\mathrm{Ca}^{2+}$ leaks. Excessive $\mathrm{Ca}^{2+}$ leakage through sparks can be disease-causing, therefore $\mathrm{Ca}^{2+}$ sparks were analyzed. For analysis, line scans of iPSC-CMs were recorded as described earlier. In each line scan, a rectangle shape area with a width of $40 \mu \mathrm{m}$ and a length of one second was analyzed with the Fiji plugin SparkMaster. The rectangle was positioned just before the start of a $\mathrm{Ca}^{2+}$ wave to obtain diastolic data. The threshold to identify sparks was set to 3.5 and the picture was split into 3 intervals by SparkMaster. Spark data were collected and ordered with excel and additional criteria to exclude small sparks were added. The used criteria were as follows:

- a minimum full width at half maximum (FWHM) of $1 \mu \mathrm{m}$

- a minimum full duration at half maximum (FDHM) of $8 \mathrm{~ms}$

- a minimum amplitude of 0.1 
The spark numbers per cardiomyocyte, the mean FWHM, the mean FDHM, and total $\mathrm{Ca}^{2+}$ leak (sum of (FWHM ${ }^{*} \mathrm{FDHM}^{\star}$ Amplitude) of all sparks in a single iPSC-CM) were included in the analysis.

\subsubsection{Immunofluorescence staining}

To visualize expressed proteins in a cellular context, immunofluorescence with targetspecific antibodies and fluorescence tagged secondary antibodies were used. Cells were washed 3 times briefly with PBS. Afterwards, cells were fixed with 4\% PFA solution for 20 min. PFA was discarded and cells were washed 3 more times with PBS. Cells were stored in $1 \%$ BSA solution until staining was performed. Cells were stained with the primary antibody diluted in $0.1 \%$ Triton-X $100 / 1 \%$ BSA in PBS and. Staining was performed overnight at $4{ }^{\circ} \mathrm{C}$. The next day, staining solution was discarded and cells were washed 3 times with PBS. Secondary antibodies were diluted in Triton-X 100/1\% BSA in PBS, added to the cells, and incubated in a moist chamber at $37^{\circ} \mathrm{C}$ for 1 hour. Subsequently, staining solution was discarded and cells were washed with PBS. For nuclear staining, cells were incubated with a 1:5000 DAPI solution in water for $10 \mathrm{~min}$ at room temperature in the dark. Cells were rinsed with PBS and water. The stained cells were mounted with fluoromount$\mathrm{G}$ on a coverslip. Images were acquired using a fluorescence microscope (Axiovert 200, Zeiss).

\subsubsection{Oil Red O staining}

To visualize lipid droplets in cells, a fatty acid stain with Oil Red $O$ is easy and efficient. The cells were incubated with Iso/Epi for $2 \mathrm{~h}$ and washed three times with PBS. Afterwards, cells were fixed with 4\% PFA for $20 \mathrm{~min}$ and washed three times with PBS. Cells were preincubated in $60 \%$ isopropanol for $5 \mathrm{~min}$ and $0.3 \%$ Oil Red O solution (in $60 \%$ isopropanol) was added. After incubation for $20 \mathrm{~min}$, cells were rinsed with tap water and stained with Mayers Hämatoxilin for $1 \mathrm{~min}$ and rinsed again with tap water. Subsequently, lipid content was semi-quantified by using threshold mapping with ImageJ 
by Dr. Isabel Schellinger to assess the lipid area expressed as percentage of total visualized area.

\subsubsection{Karyotyping}

For karyotyping, cells were incubated with Demecolcemid $100 \mathrm{ng} / \mathrm{ml}$ for $12-16 \mathrm{~h}$. The supernatant was collected and cells were rinsed with PBS. Cells were digested with TrypLE for $45 \mathrm{~s}$, harvested, and pooled. Cells were centrifuged at $200 \mathrm{~g}$ for $5 \mathrm{~min}$. Afterwards, most of the supernatant was discarded and cells were carefully resuspended in the remaining supernatant. $6-8 \mathrm{ml}$ pre-warmed $37^{\circ} \mathrm{C} 0.075 \mathrm{M} \mathrm{KCl}$ solution was added dropwise up to $8 \mathrm{ml}$ to the cells while constantly shaking the tube. The cells were incubated at $37{ }^{\circ} \mathrm{C}$ for $45 \mathrm{~min}$. The solution was centrifuged for $5 \mathrm{~min}$ at $200 \mathrm{~g}$, most of the supernatant was discarded and the cells were resuspended in the remaining $500 \mu \mathrm{l}$ supernatant. Cooled methanol-acetone solution (3:1) was added dropwise under constant shaking up to $5 \mathrm{ml}$ and the solution was incubated on ice for $10 \mathrm{~min}$. The solution was centrifuged for 3 minutes at $200 \mathrm{~g}$. This fixation step was repeated two more times. After fixation, cells were resuspended in $2 \mathrm{ml}$ methanol-acetone solution and dropped from 50 $\mathrm{cm}$ height on prepared microscope slides. The cell spreads were allowed to dry overnight. Chromosomes were stained in giemsa solution (1:20 in water) for 5 min at RT. Cells were rinsed several times with water and allowed to dry. Images were acquired by the Zeiss Axio Imager M2 light microscope. Karyotypes were analyzed with the Case Data Manager 6.0 software (Applied Spectral Imaging, ASI).

\subsubsection{Multi-electrode arrays}

IPSC-CMs were analyzed for their electrical properties with MEAs (Microchannel Systems, Reutlingen, Germany). MEAs were stored in water and made sterile by incubation in hot $\left(>70^{\circ} \mathrm{C}\right)$ water and subsequent UV light illumination for up to $1 \mathrm{~h}$. MEAs were coated with a small drop of geltrex on the electrode area. IPSC-CMs were digested with $0.25 \%$ Trypsin/EDTA and $1 \times 10^{5}$ (one-well MEAs) or $2.5 \times 10^{4}$ (6-well MEAs) were 
plated in $100 \mu \mathrm{l}$ cardio-digest medium on the coated MEAs. Cells were allowed to regenerate for at least 3 days in cardio-culture medium. Measurements were conducted at $37^{\circ} \mathrm{C}$ and Iso or Epi were added 1:100 in a cumulative manner to achieve the target concentrations. After an equilibration time of $5 \mathrm{~min}$ after each drug, electrical signals were recorded for $5 \mathrm{~min}$ with MC_Rack (Multichannel Systems). For each experiment, one electrode with a strong field potential was chosen and beating rates and field potential duration (FPD) were extracted from the Data with MC_Rack. Further analysis was carried out with Excel (Microsoft Corporation) and data were visualized with GraphPad Prism (Graph Pad Software). Frequency corrected FPD (FPDcF) was calculated using Fridericia's Formula to correct the decreasing FPD during increased frequencies: $F P D C F=\frac{F P D}{(\text { interspike intervall }[s])^{\frac{1}{3}}}$ (Fridericia, 1920).

Conduction velocity data were recorded on the MEA system with the software cardio2D (Multichannel Systems, Germany) using different catecholamine concentrations. Data were analyzed using cardio2D+ software (Multichannel Systems, Germany), MultiElec, Excel, and Graph Pad.

\subsubsection{Statistical analysis}

Data were given as the mean \pm SEM (standard error of the mean). The unpaired Student's $t$-test was used to compare the differences between two independent groups. For significance analysis, the multiple $t$-test, the one-way and two-way ANOVA were used for comparison of more groups and conditions and corrected for multiple comparisons. For dose-response curves, significances were calculated by extra sum-of-squares $F$ test. $A$ $p$-value of less than 0.05 was considered statistically significant with ${ }^{*}=p<0.05,{ }^{* *}=p<0.01$ and ${ }^{* * *}=p<0.001$. Statistical analysis was performed with GraphPad prism. N-numbers were defined as differentiation experiments of different cell lines from mentioned patients/controls. Alternatively, $n$-numbers are defined as number of single cells used in the experiment. 


\section{Results}

\subsection{Clinical profile of recruited TTS-patients}

The patients who were recruited from the Takotsubo registry in Zurich for this study fulfilled the Mayo-clinic criteria for TTS. Somatic cells from 7 recruited probands (4 TTS patients and 3 controls) were successfully isolated. These subjects had the following clinical profiles:

Table 20: Recruited patients and healthy probands and underlying conditions

\begin{tabular}{|c|c|c|c|c|c|c|c|c|}
\hline $\begin{array}{l}\text { TTS- } \\
\text { patie } \\
\text { nt }\end{array}$ & $\begin{array}{l}\text { Age } \\
\text { at } \\
\text { TTS }\end{array}$ & Sex & $\begin{array}{l}\text { Triggering } \\
\text { factors }\end{array}$ & $\begin{array}{l}\text { Acute } \\
\text { psychiatr } \\
\text { ic } \\
\text { disorder }\end{array}$ & $\begin{array}{l}\text { Acute } \\
\text { neurologi } \\
\text { c } \\
\text { disorder }\end{array}$ & $\begin{array}{l}\text { TTS } \\
\text { type }\end{array}$ & LVEF in \% & $\begin{array}{l}\text { NYH } \\
\text { A }\end{array}$ \\
\hline 1-TTS & 60 & Female & $\begin{array}{l}\text { Emotional } \\
\text { stress: } \\
\text { grief and loss }\end{array}$ & yes & no & apical & 40 & IV \\
\hline 2-TTS & 69 & Female & $\begin{array}{l}\text { Emotional } \\
\text { stress: } \\
\text { Dispute with } \\
\text { husband }\end{array}$ & no & no & apical & 40 & I \\
\hline 5-TTS & 67 & Female & $\begin{array}{l}\text { Physical } \\
\text { stress: } \\
\text { seizure with } \\
\text { vigilance }\end{array}$ & no & yes & apical & 40 & I \\
\hline 8-TTS & 72 & Female & $\begin{array}{l}\text { Emotional } \\
\text { stress: fear } \\
\text { Physical } \\
\text { stress: } \\
\text { seizure }\end{array}$ & no & yes & apical & 40 & I \\
\hline $\begin{array}{l}\text { Healt } \\
\text { hy } \\
\text { contr } \\
\text { ol }\end{array}$ & Age & Sex & $\begin{array}{l}\text { Triggering } \\
\text { factors }\end{array}$ & $\begin{array}{l}\text { Acute } \\
\text { psychiatr } \\
\text { ic } \\
\text { disorder }\end{array}$ & $\begin{array}{l}\text { Acute } \\
\text { neurologi } \\
\text { c } \\
\text { disorder }\end{array}$ & $\begin{array}{l}\text { TTS } \\
\text { type }\end{array}$ & LVEF in \% & $\begin{array}{l}\text { NYH } \\
\text { A }\end{array}$ \\
\hline $1-C$ & 52 & Female & - & - & - & - & - & - \\
\hline
\end{tabular}




\begin{tabular}{|l|l|l|l|l|l|l|l|l|}
\hline $2-\mathrm{C}$ & 25 & Female & - & - & - & - & - & - \\
\hline $3-\mathrm{C}$ & 25 & Female & - & - & - & - & - & - \\
\hline iFB2 & - & Female & - & - & - & - & - & - \\
\hline
\end{tabular}

The TTS group consisted of four female patients with an age range of 60-72 years. Somatic cells of all 4 TTS patients were reprogrammed into iPSCs in this thesis. Our control group consisted of four healthy females aged 25-52 years, from whom somatic cells of 3 patients were reprogrammed into iPSCs in this thesis. One of the CTRL-iPSC lines (iFB2) already existed in our lab and was described before (Streckfuss-Bömeke et al., 2013).

\subsection{IPSC production and characterization}

\subsubsection{Production of iPSCs from fibroblasts and blood PBMCs}

Fibroblasts were obtained from proband's skin punch biopsies by mechanical dissection followed by fibroblast outgrowth from the biopsy (Figure 5A). Fibroblasts showed spindleshaped morphology (Figure $5 A$ ). PBMCs were isolated from full blood samples and showed typical round morphology (Figure 5A).

For the generation of iPSCs, fibroblasts and PBMCs were reprogrammed integration-free by using Sendai viruses or episomal plasmids encoding for an enhanced set of transcription factors. SOX2, KLF4, L-MYC, LIN28, OCT 3/4, and sh-RNA against P53 were encoded by the plasmids (Okita et al., 2011). SOX2, KLF4, C-MYC, and OCT3/4 were transported by the Sendai virus. After four weeks in culture, several iPSC-colonies grew from the original cells. The iPSC-clones were selected, mechanically picked, and expanded. From each patient and control proband, two cell lines were chosen for expansion and characterization. The obtained cell lines were subsequently used for cardiomyocyte production and experiments. 


\subsubsection{IPSCs show stem cell morphology and express pluripotency markers}

To prove the pluripotency of the produced iPSC-lines, colony morphology, alkaline phosphatase expression, and pluripotency marker expression on mRNA and protein levels were analyzed. IPSC-colonies showed a typical ESC-like round morphology and were stained positive for alkaline phosphatase (Figure 5B), an enzyme, which is active in undifferentiated cells (O'Connor et al., 2008), while feeder cells remained negative (Figure 5B). On mRNA-level, the produced iPSCs revealed strong expression of OCT4, NANOG, SOX2, LIN28, GDF3, and FOXD3 (Figure 5C). The source fibroblasts and PBMC lines did not show such an expression (Figure $5 \mathrm{C}$ ). At protein level, the nuclear-localized transcription factors OCT4, NANOG, SOX2 (Figure 6, red), and the membrane-bound pluripotency markers TRA-1-60 and SSEA4 (Figure 6, green) were highly expressed in all tested iPSCs. Furthermore, the pluripotency marker LIN28 (Figure 6, red) was found to be localized both in the cytoplasm and nucleus of the stained cell lines. No differences in pluripotency between the different cell lines of TTS- and CTRL-iPSCs were observed. 
A
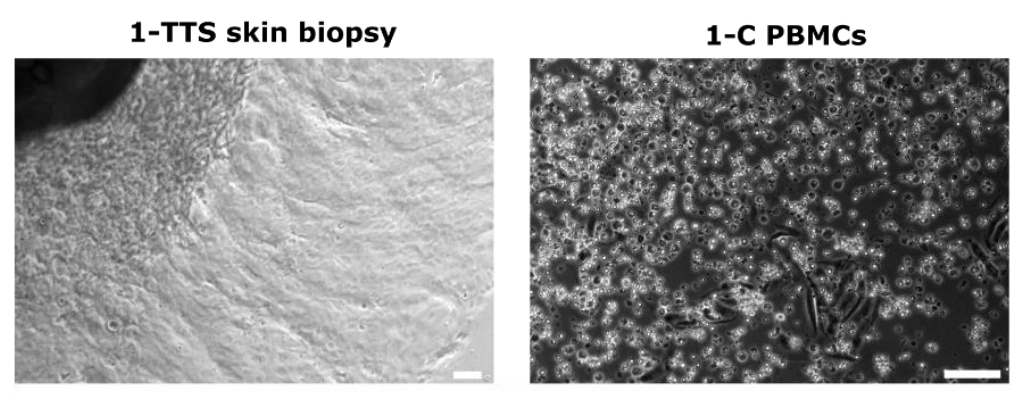

B
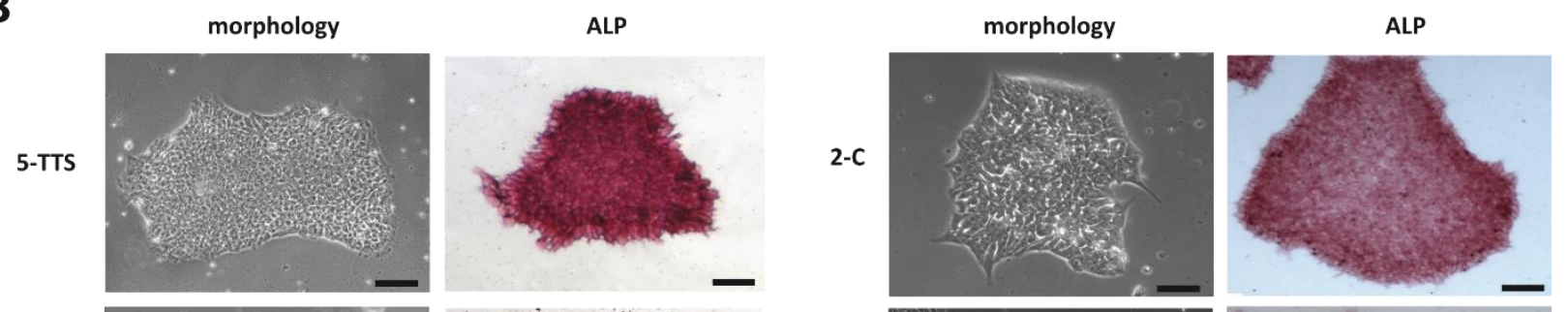

8-TTS
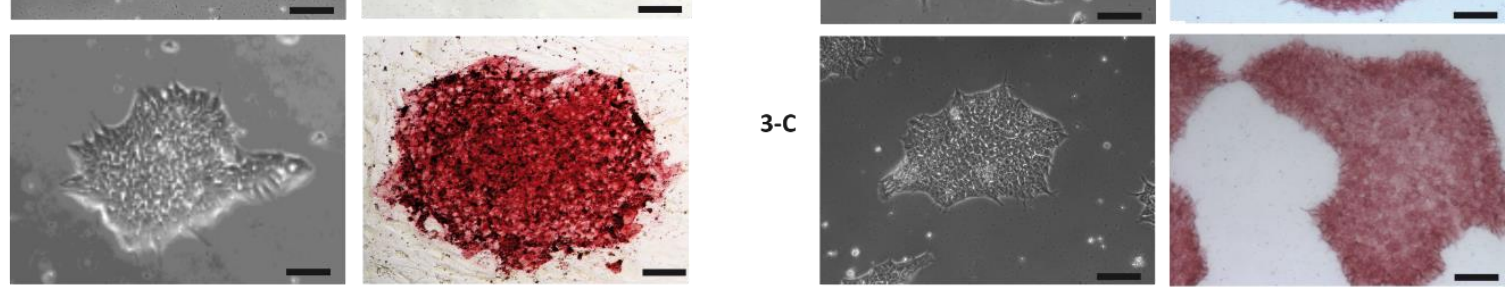

C
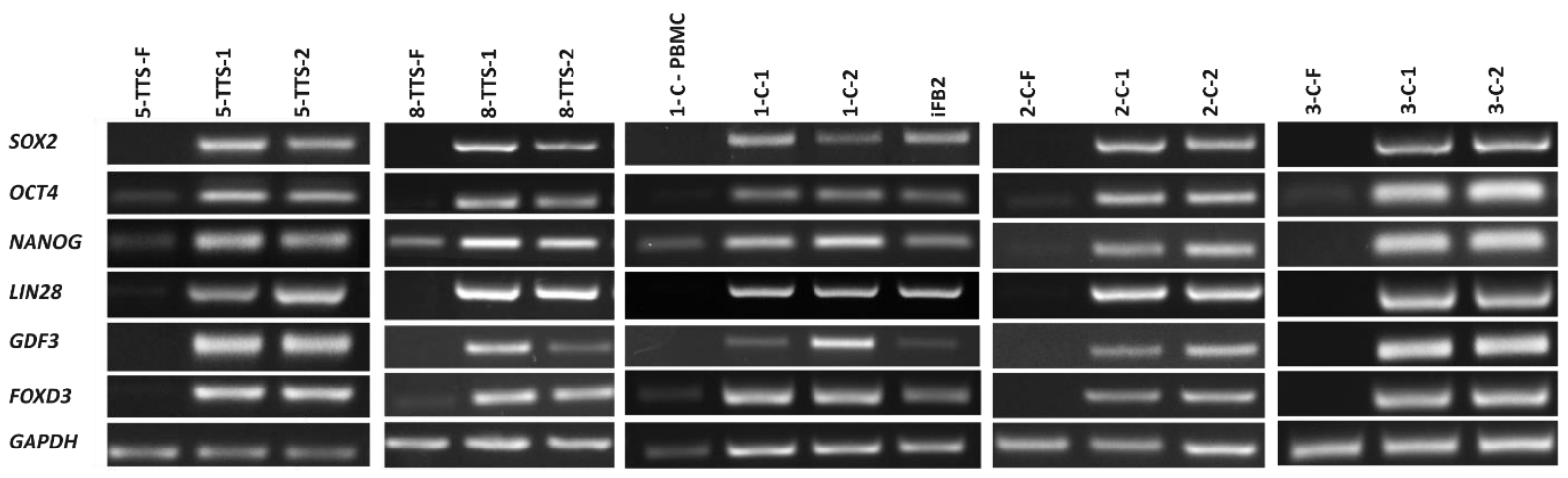

Figure 5: Reprogramming of somatic cells to iPSCs: A: Brightfield images of the source material for the production of iPSCs. Skin fibroblasts growing out of a skin punch biopsy (left picture). blood mononuclear cells (PBMCs) isolated from blood samples (right picture). Scale bars $=100 \mu \mathrm{m} \mathrm{B}$ : IPSC-colonies showed a typical ESC-like morphology and were stained positive for alkaline phosphatase (ALP) (red). Scale bars $=100 \mu \mathrm{m}$ C: IPSCs express the pluripotency markers SOX2, OCT4, NANOG, LIN28, GDF3, FOXD3 on mRNA level. GAPDH was used as loading control. 


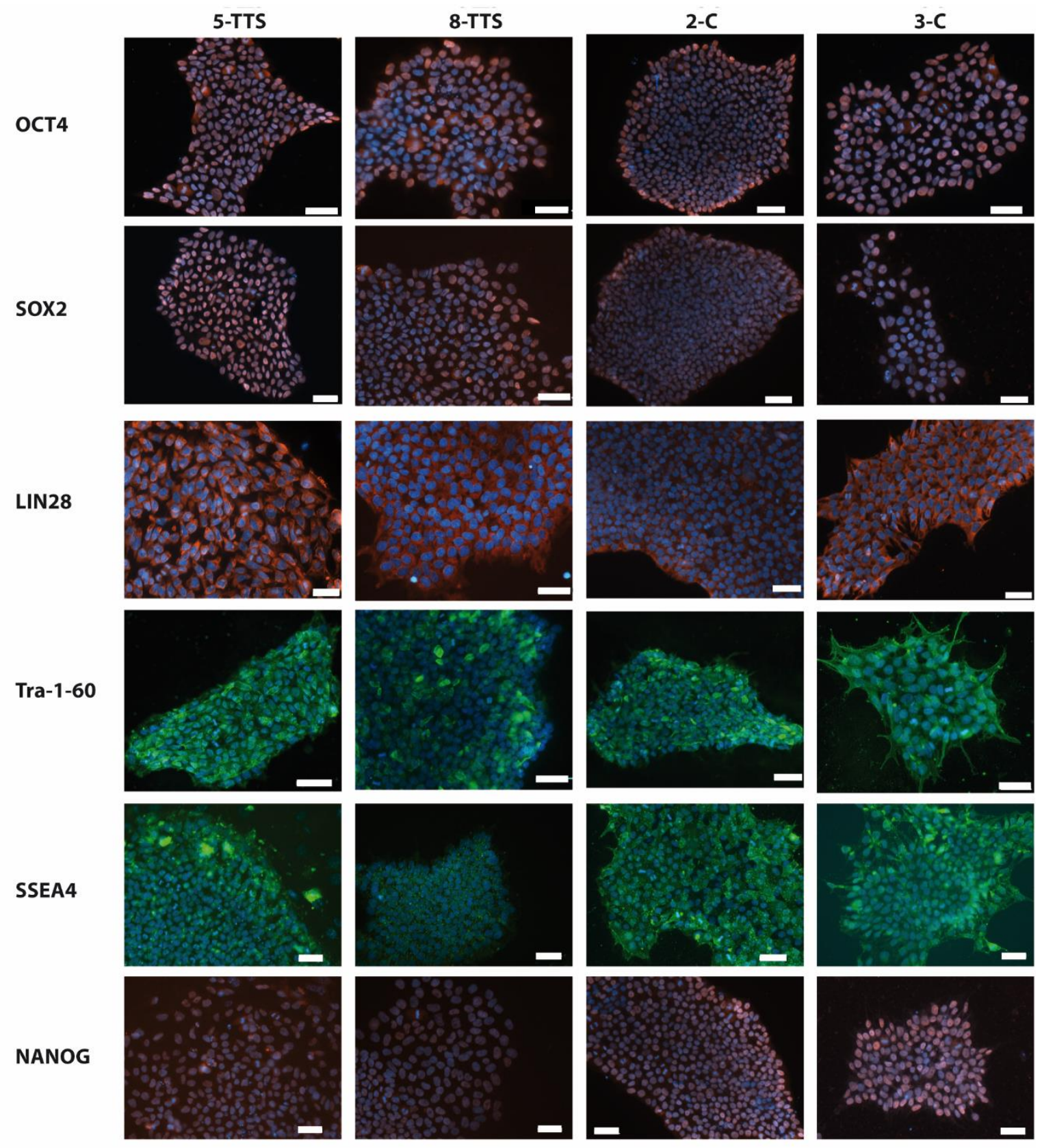

Figure 6: 5-TTS, 8-TTS, 2-C and 3-C-iPSCs show expression of pluripotency markers on protein level. Immunofluorescence staining of OCT4 (red), SOX2 (red), LIN28 (red), TRA-1-60 (green), SSEA4 and (green) NANOG (red). Nuclei were stained with DAPI (blue). Scale bars=50 $\mu \mathrm{m}$.

4.2.3 iPSCs can be differentiated into cells of all three germ layers 
One key characteristic of pluripotent stem cells is their ability to differentiate into cells of the three different germ layers, namely endoderm, mesoderm, and ectoderm. In order to confirm this, differentiation tests were carried out in vitro and in vivo.

For in vitro differentiation, mass culture assays were used. In brief, clumps of iPSCs were spontaneously differentiated into all possible directions. IPSCs were grown in aggregates in suspension culture for 8 days and then allowed to grow on plates for up to 25 more days. The resulting cultures were analyzed via immunofluorescence. On protein levels, AFP (red) was detected as an early endodermal marker, $\beta$-tubulin (green) as an ectodermal marker, and $\alpha$-SMA (green) as a mesodermal marker (Figure 7A). 
A

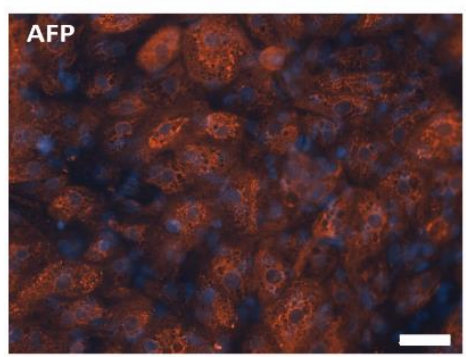

a-SMA

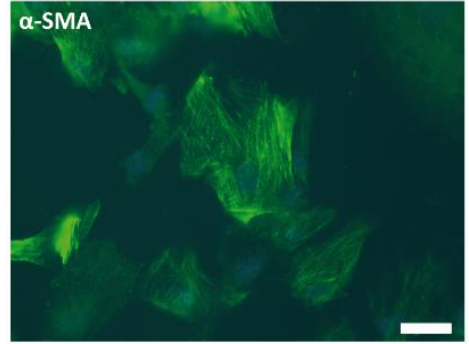

$\beta$-tubulin

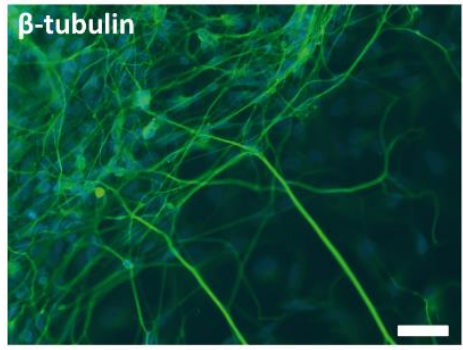

B
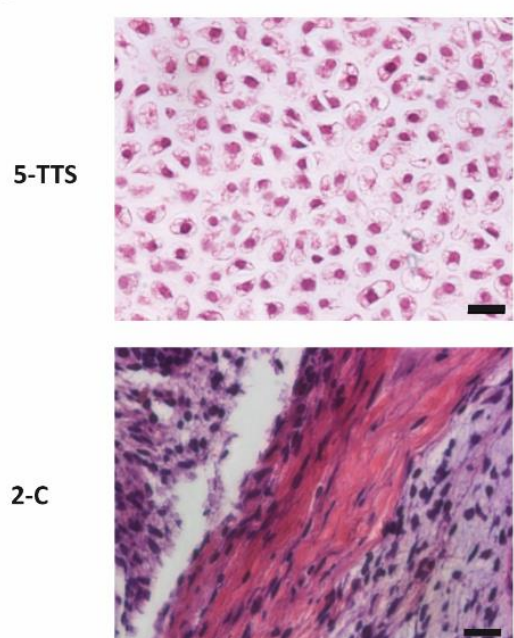

8-TTS
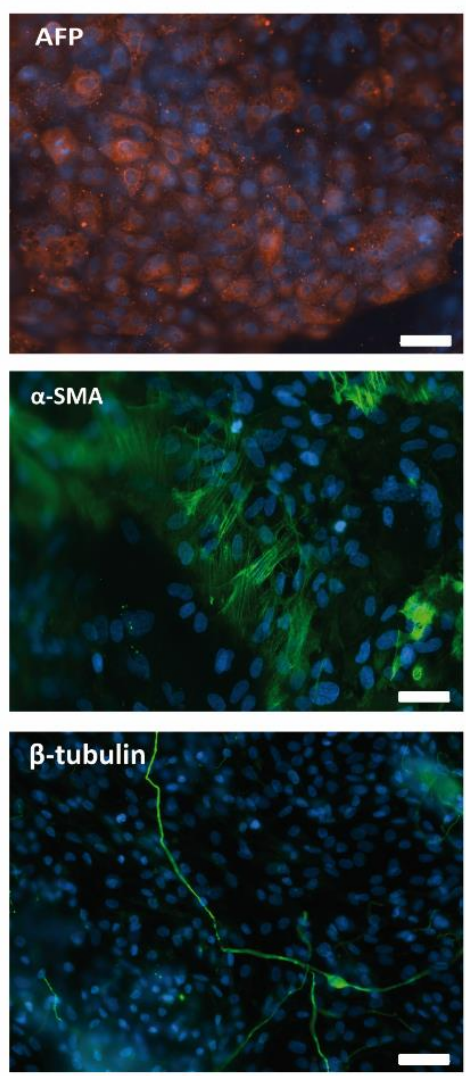

Endoderm
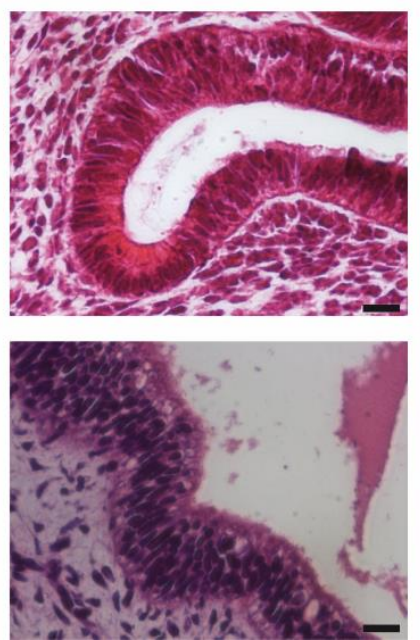

2-C
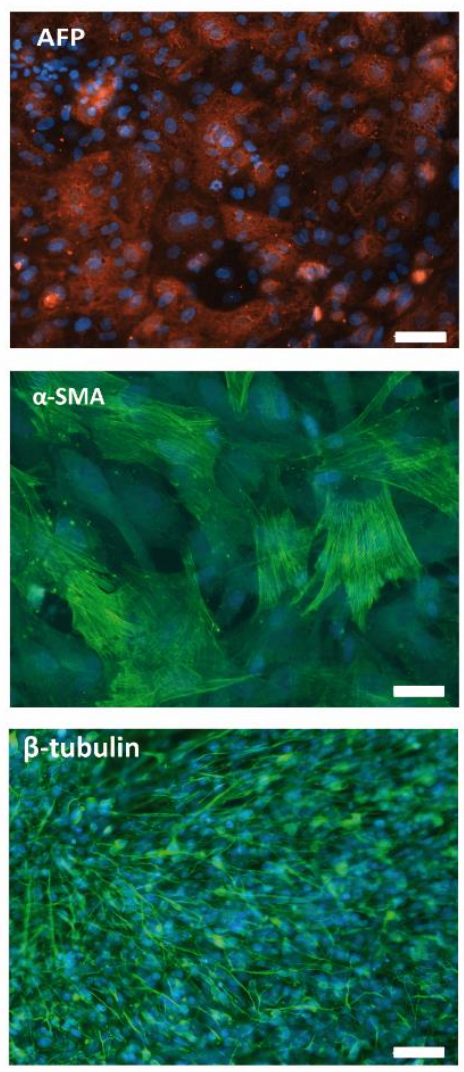

Ectoderm
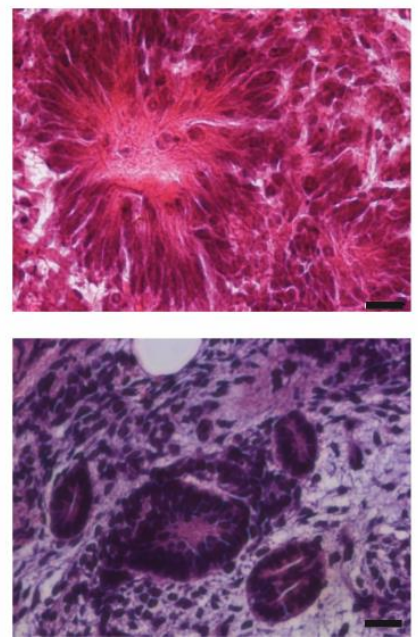

Figure 7: IPSCs differentiated to all three germ layers in vitro and in vivo. A: In vitro differentiated cells express the endodermal marker AFP (red), the mesodermal marker $\alpha$-SMA (green), and the ectodermal marker $\beta$-tubulin (green). In the case of $\beta$-tubulin, cells exhibiting a typical neuronal morphology. Nuclei were stained with DAPI (blue). Scale bars: $50 \mu \mathrm{m}$. B: In vivo teratoma formation shows cells from mesoderm (cartilage, muscle), endoderm (intestine epithelium), and ectoderm (neuronal rosettes). Scale bars $=50 \mu \mathrm{m}$. 
In an in vivo teratoma forming assay, the iPSCs were injected into immunosuppressed SCID mice. Growing teratomas were explanted (injection and explantation were done by Prof. Ralf Dressel) and histologically analyzed for tissue characteristics of the three different germ layers. IPSCs were able to differentiate into mesoderm, as shown by the formation of cartilage tissue (Figure 7B), endoderm as shown by the formation of intestine tissue (Figure 7B) and ectoderm as shown by the formation of neuronal rosettes (Figure 7B).

To ensure none of the cells harbored an HIV infection, all cell lines were screened by our group. All generated iPSC lines were tested negatively for HIV infection by using a high throughput technology in our lab (Hübscher et al., 2019).

In summary, in this thesis, 4 TTS patients and 3 controls were integration-free reprogrammed into iPSC-lines by Sendai virus transduction or plasmid transfection. The three iPSC lines (2-C, 5-TTS, 8-TTS) analyzed for pluripotency showed a typical ESC-like morphology and expressed alkaline phosphatase. Additionally, the iPSCs showed robust expression of pluripotency markers on mRNA and protein levels. Furthermore, the cells were able to differentiate into all three germ layers in vitro and in vivo. No relevant differences were detected between CTRL- and TTS-iPSCs regarding spontaneous differentiation capacity or pluripotency marker expression. Of note, all tested cell lines were free of HIV.

\subsubsection{IPSCs can be directly differentiated into functional cardiomyocytes}

To produce iPSC-CMs, targeted Wnt-pathway manipulations were used (Burridge et al., 2014). To achieve this, the GSK3-inhibitor CHIR was added to the medium for two days to achieve mesoderm induction and the Wnt-inhibitor IWP2 was used for additional two days to induce cardiomyocyte differentiation. The differentiated cardiomyocytes were metabolically selected with lactate to achieve a high purity (Burridge et al., 2014). IPSCCMs were allowed to mature during a cultivation period of at least two months. 


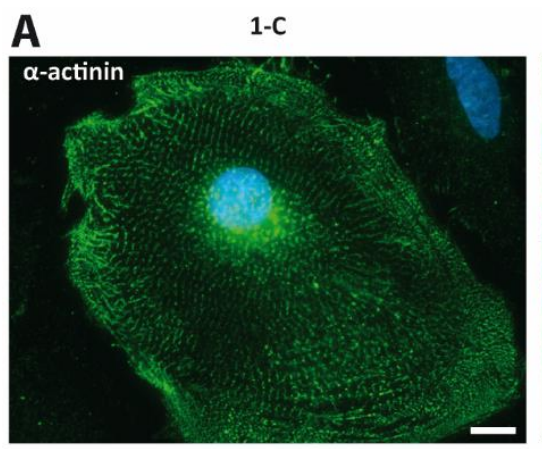

B
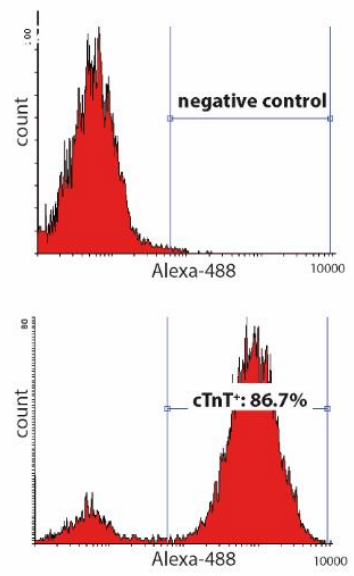

D

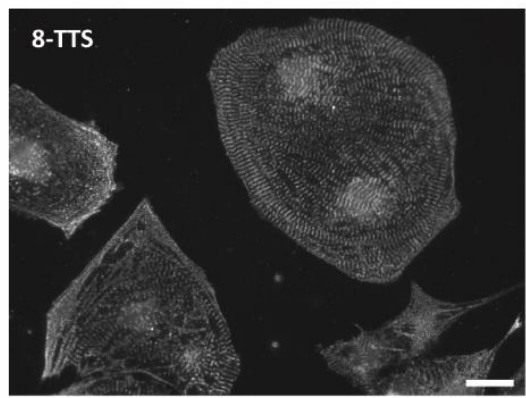

5-TTS

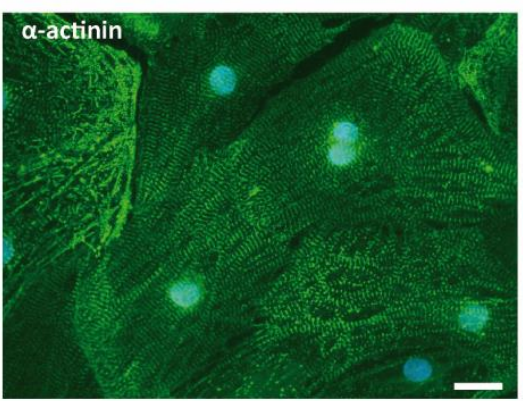

C
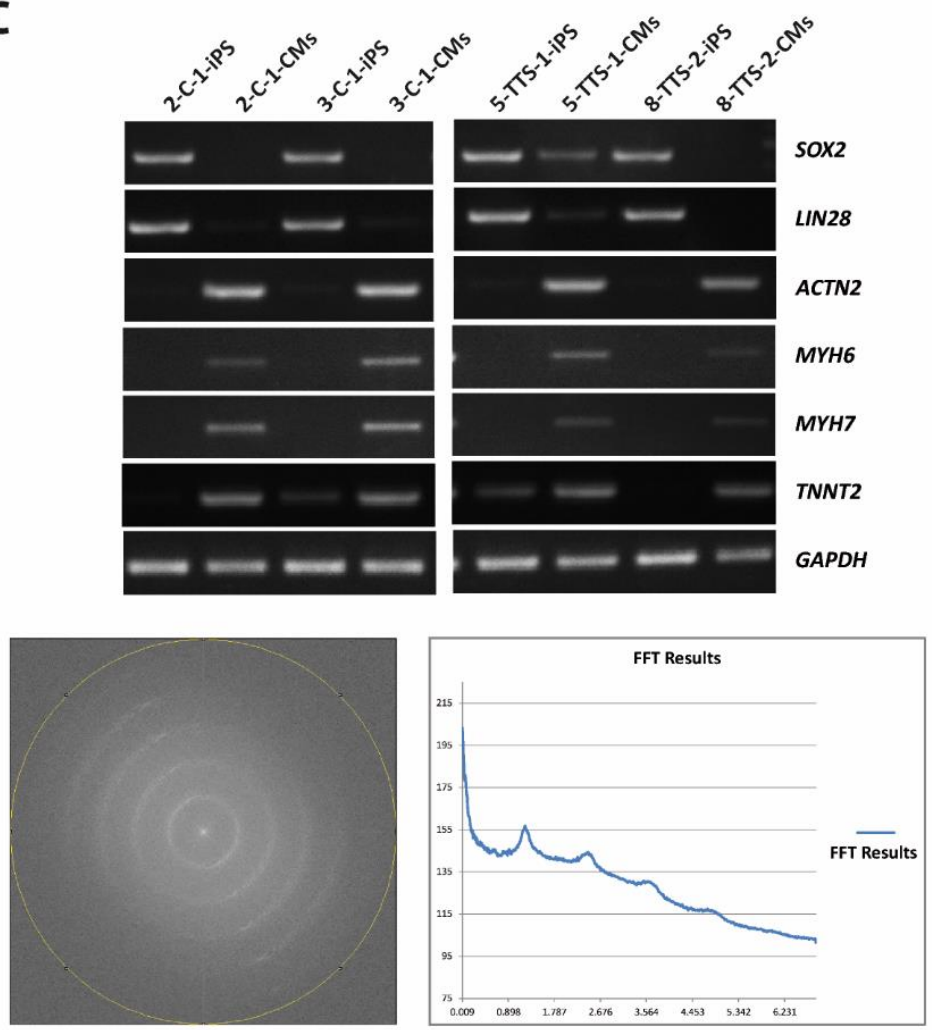

Figure 8: Cardiac differentiation of iPSCs. A: Differentiated cardiomyocytes express a-actinin (green) on protein level as analyzed by immunofluorescence. Nuclei were stained with DAPI (blue). Scale bars $=20 \mu \mathrm{m}$ B: Example of FACS analysis of cTNT-positive cells. Overall, iPSCCMs showed a purity of over $80 \%$. C: Differentiated cardiomyocytes express the cardiomyocyte markers ACTN2, MYH6, MYH7, and TNNT2 on mRNA level. D: Analysis of the sarcomeric structure with fast Fourier transformation (FFT) revealed a regular sarcomeric structure. Left picture: $\alpha$-actinin staining of a cardiomyocyte used for FFT analysis. Middle picture: FFT of the left picture. Right picture: Intensity plot of the FFT in the middle shows regular peaks representing different sarcomere distances.

The produced iPSC-CMs exhibited a prominent sarcomeric structure, as shown by $\alpha-$ actinin staining (Figure 8A, green). To analyze the amount of generated iPSC-CMs, 2month-old cells were stained for the cardiac marker cTnT and analyzed by means of flow 
cytometry. The obtained iPSC-CMs showed a purity of $80-95 \%$ (Figure 8B). The cardiomyocytes expressed the typical cardiomyocyte markers a-actinin (ACTN2), $\alpha$ myosin heavy chain (MYH6), $\beta$-myosin heavy chain (MYH7), and cTNT (TNNT2) with simultaneous depletion of the pluripotency marker SOX2 and LIN28 on mRNA level (Figure $8 \mathrm{C}$ ). No differences in cardiac marker expression and differentiation capacity were observed between CTRL-iPSC-CMs and TTS-iPSC-CMs. Furthermore, the differentiated iPSC-CMs showed robust regular beating activity, often in a coordinated manner, exhibited strong $\mathrm{Ca}^{2+}$ transients, and regular electrical activity.

\subsection{Stress can be induced by the addition of catecholamines}

To uncover whether catecholamines can induce stress in iPSC-CMs, they were challenged with different catecholamine dosages (iso $100 \mathrm{nM}-5 \mathrm{mM}$, Epi $100 \mathrm{nM}-500$ $\mu \mathrm{M})$ and sarcomeric structure and apoptosis were analyzed.

Our group showed that Nuclear Receptor Subfamily 4 Group A Member 1 (NR4A1) was increased upon Iso stimulation as shown by next-generation sequencing. The same was observed in qPCR studies in reaction to Epi and Iso as a consequence of the increase in cellular stress. TTS-iPSC-CMs showed a stronger increase of NR4A1 expression in response to Iso than CTRL-iPSC-CMs. When challenged with catecholamines, the sarcomeric structure, as demonstrated by a-actinin staining, was not disturbed (Figure 9A).

To check for cytotoxic effects of high levels of catecholamines, apoptosis was analyzed by annexin V/propidium iodide staining and subsequent quantification. Apoptosis levels reached around $4 \%$ for untreated iPSC-CMs. For iPSC-CMs treated with low (100 nM) or high levels (5 mM Iso or $500 \mu \mathrm{M} \mathrm{Epi)} \mathrm{of} \mathrm{catecholamines} \mathrm{(Figure} \mathrm{9B),} \mathrm{no} \mathrm{significant}$ differences to untreated cells were observed. Additionally, no differences were found between CTRL-iPSC-CMs and TTS-iPSC-CMs. In summary, Iso and Epi treatment had no acute cytotoxic effects on iPSC-CMs. 


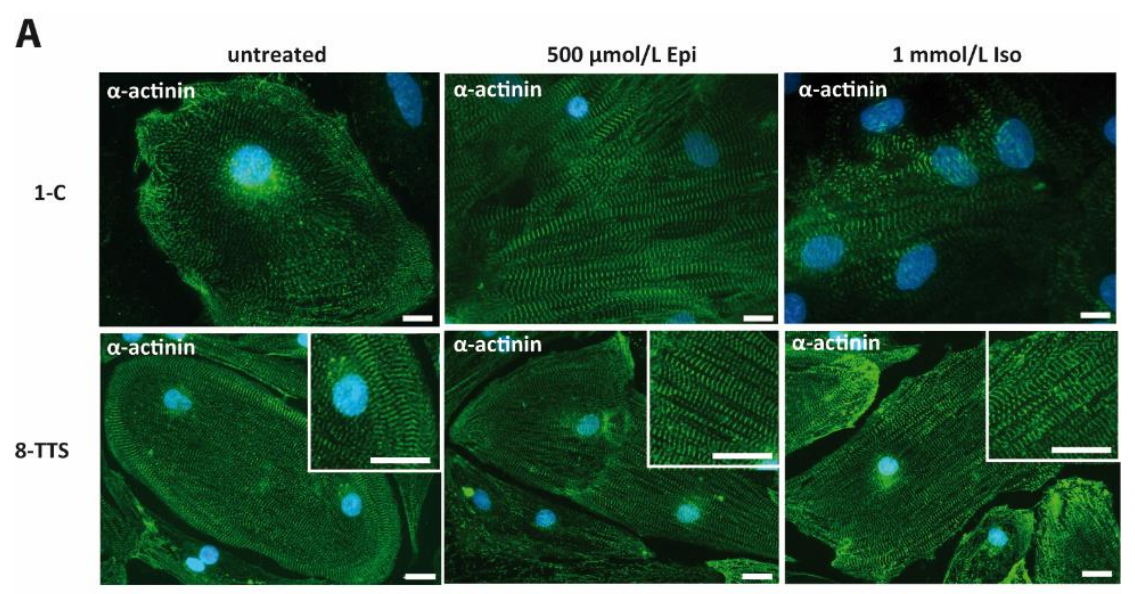

B
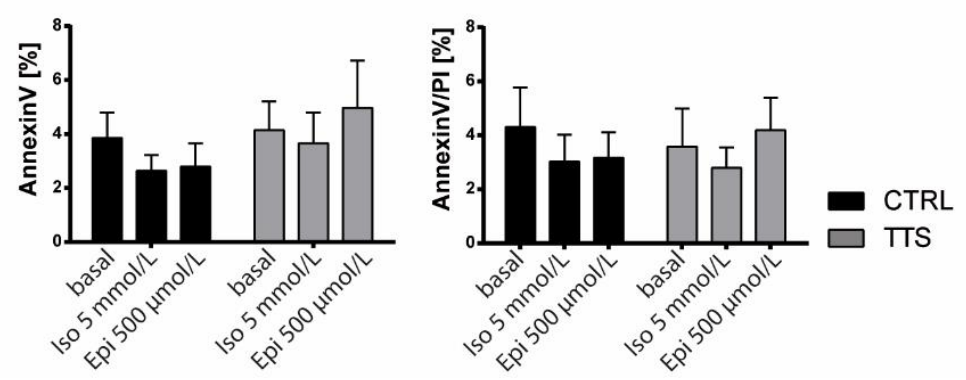

Figure 9: Stress induction in cardiomyocytes by addition of high dosages of catecholamines. A: a-actinin staining shows that treatment with high levels of catecholamines did not result in an altered sarcomeric structure. Scale bar: $20 \mu \mathrm{m}$ B: Apoptosis measurements with annexin $\mathrm{V} /$ propidium iodide (PI) showed no increased apoptosis in catecholamine treated CTRL-iPSCCMs ( $n=4$ differentiations: $1-C 1[n=1]), 2-C[n=1]$ and iFB2 $[n=2])$ or TTS-iPSC-CMs $(n=3$ differentiations: 1 -TTS [ $n=1], 5$-TTS [ $n=1$ ], and 8 -TTS [ $n=1]$ ). Data are presented as mean \pm SEM. Significances were tested using one-way ANOVA.

\subsection{Catecholamines cause electrical disturbances}

Earlier experiments from our group revealed different CAMP responses in CTRL-iPSCCMs and TTS-iPSC-CMs: CTRL-iPSC-CMs reached maximum CAMP concentrations after stimulation with $100 \mathrm{nM}$ Iso, whereas in TTS-iPSC-CMs, cAMP concentration reached their peak at Iso dosages of $1 \mathrm{mM}$ (Borchert et al., 2017). To examine whether this also causes changes in electrical behavior, multi-electrode arrays were used to observe the electrical properties of CTRL-iPSC-CMs and TTS-iPSC-CMs. Therefore, the iPSC-CMs were stimulated with increasing dosages of catecholamines (Iso and Epi). 
Beating frequency, field potential duration, FPDcF, and cardiac conduction velocity were calculated.

Figure 10A shows some representative MEA traces of CTRL- and TTS-iPSC-CM preparations with and without Iso stimulation. Generally, the addition of Iso caused a frequency increase as expected. However, some iPSC-CMs reacted with silenced electrical activity towards stimulation. Interestingly, the observed silencing effect was very acute without strong preceding arrhythmias (Figure 10A).

TTS-iPSC-CMs reached a frequency maximum at $1 \mu \mathrm{M}$ and CTRL-iPSC-CMs at higher levels of $10 \mu \mathrm{M}$. After reaching their maximal frequency, TTS- and CTRL-iPSC-CMs showed decreasing beating frequencies at rising Iso concentrations, reaching a minimum at Iso levels of $5 \mathrm{mM}$ (Figure 10B). TTS-iPSC-CMs showed a stronger reaction towards Iso by exhibiting a significantly increased relative beating frequency compared to CTRLiPSC-CMs at $1 \mu \mathrm{M}$ (3.3-fold \pm 0.3 vs. 2.0 -fold \pm 0.3$)$ and $10 \mu \mathrm{M}(3.3 \pm 0.2$ fold vs. $2.3 \pm 0.3$ fold) (Figure 10C).

As previously mentioned, some of the cardiomyocytes showed a cessation of beating upon Iso addition. In the conducted experiments, TTS-iPSC-CMs were more prone to this cessation of beating caused by high levels of Iso than the observed CTRL-iPSC-CMs (Figure 10D). Surprisingly, Epi did not cause beating cessation. Removal of Iso and recovery of the cells for $2-24 \mathrm{~h}$ hours led to a reversal of the described effects (Figure 10A). Surprisingly, Epi did not cause electrical silencing in TTS- or CTRL-iPSC-CMs. 
A

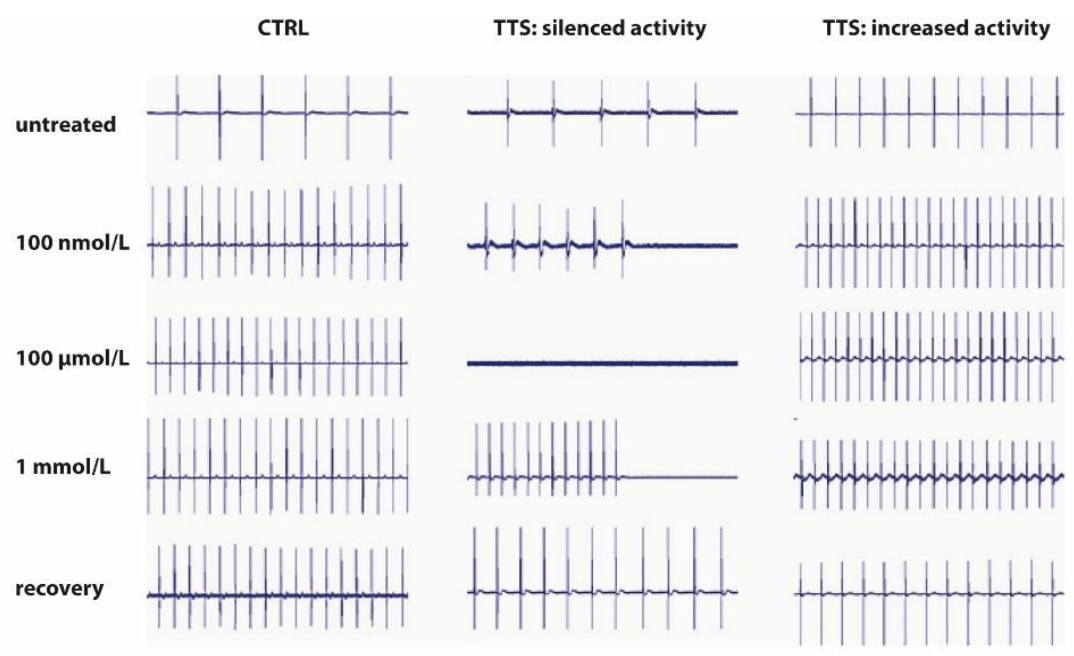

B

C

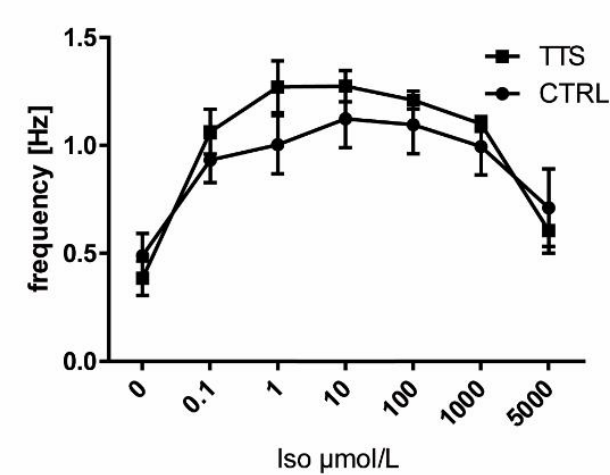

D

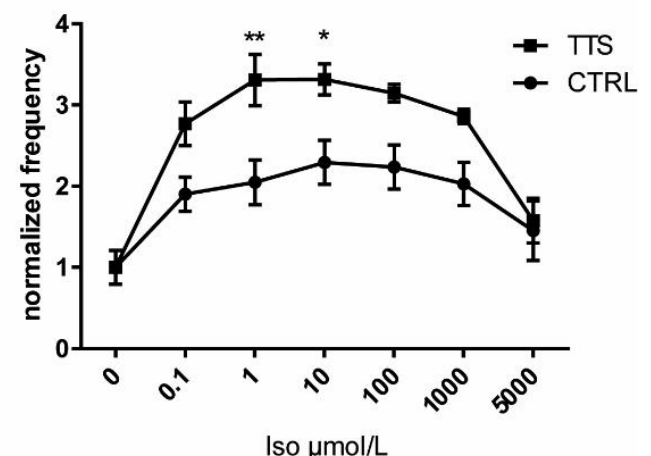

Iso $\mu \mathrm{mol} / \mathrm{L}$

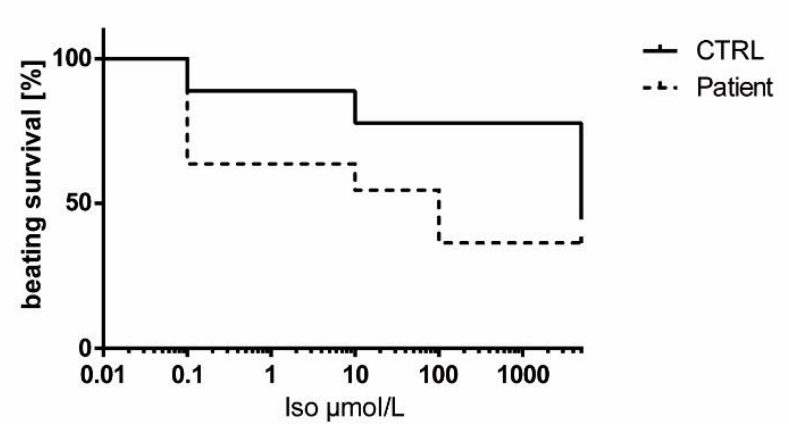

Figure 10: Multi-electrode arrays uncover electrical differences between TTS- and CTRL-iPSCCMs. A: Representative MEA traces of CTRL- and TTS-iPSC-CMs. Of note are the spontaneous disruption of beating activity (middle) and the increased beating activity in the non-disrupted iPSCCMs (right). B: CTRL-iPSC-CMs ( $n=8$ MEA experiments: $1-C[n=6], 2-C[n=1]$, and iFB2 [ $n=1]$ ) and TTS-iPSC-CMs ( $n=7$ MEA experiments: $1-T T S[n=6]$ and 8-TTS $[n=1])$ beating frequency under Iso stimulation. C: Normalized beating frequency analysis of CTRL-iPSC-CMs ( $n=8$ MEA experiments: $1-C[n=6], 2-C[n=1]$, and iFB2 $[n=1])$ and TTS-iPSC-CMs $(n=7$ MEA experiments: 1-TTS [ $n=6]$ and 8-TTS [ $=1]$ ) under Iso stimulation. D: Quantification of silenced electrical activity of CTRL-iPSC-CMs ( $n=8$ MEA experiments: $1-C[n=6], 2-C[n=1]$, and iFB2 
$[n=2])$ and TTS-iPSC-CMs ( $n=7$ MEA experiments: 1 -TTS $[n=8]$ and 8-TTS $[n=3])$ after Iso challenge presented as a beating survival curve. TTS-iPSC-CMs stopped electrical activity at lower Iso dosages than CTRL-iPSC-CMs. All data are presented as mean \pm SEM. Significant differences were calculated using two-way ANOVA.

Field potential durations provide information about action potential durations. Analysis of the field potential duration (Figure $11 \mathrm{~A} / \mathrm{C}$ ) and the FPDcF (algorithm of Frederica) (Figure $11 \mathrm{~B} / \mathrm{D}$ ) revealed no significant differences between TTS and CTRL-iPSC-CMs (Figure 11). Moreover, FPD decreased upon Iso and Epi treatment (Figure 11A/C). FPDcF stayed constant during Iso (Figure 11B) and Epi (Figure 11D) treatment.
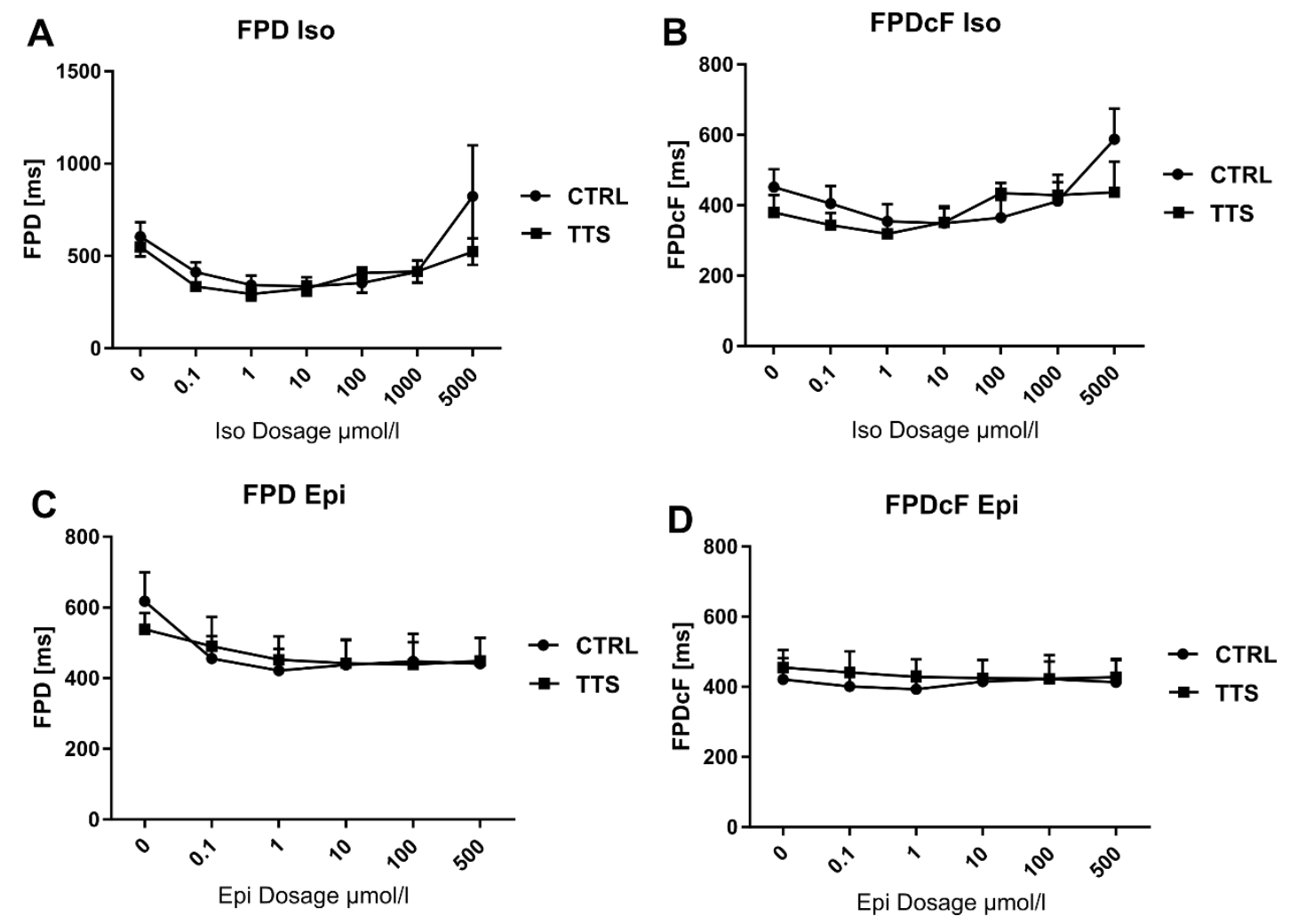

Figure 11: Measurement of field potential duration (FPD) and frequency corrected field potential durations (FPDcF) with multi-electrode arrays uncover no significant differences between CTRL and TTS-iPSC-CMs. A: Field potential durations of CTRL- $(n=7$ MEAs: $1-C[n=6]$, and iFB2 $[n=1])$ and TTS-iPSC-CMs ( $n=7$ MEAs: 1 -TTS $[n=6]$ and 8-TTS $[n=1])$ stimulated with Iso. B: Frequency corrected field potential durations of CTRL- $(n=7$ MEAs: $1-C[n=6]$, and iFB2 $[n=1])$ and TTS-iPSC-CMs ( $n=7$ MEAs: 1 -TTS $[n=6]$ and 8-TTS $[n=1])$ challenged with Iso. C: Field potential durations of CTRL- $(n=8$ MEAs: $1-C[n=6]$ and iFB2 $[n=2])$ and TTS-iPSC-CMs $(n=6$ MEAs: 1-TTS [ $=4$ ] and 8-TTS [ $=2$ ]) stimulated with Epi. D: Frequency corrected field potential durations of CTRL- $(n=8$ MEAs: $1-C[n=6]$ and iFB2 $[n=2])$ and TTS-iPSC-CMs ( $n=6$ MEAs: 1 TTS $[n=4]$ and 8 -TTS $[n=2]$ ) challenged with Epi. Data are presented as mean \pm SEM. Significances were calculated by two-way ANOVA. 
To examine whether electrical coupling between iPSC-CMs is aberrant, conduction velocity measurements were carried out. Conduction velocity was calculated using cardio $2 \mathrm{D}$ and Multi Elec by analyzing the time differences between maximum peaks at different electrodes.

Figure $12 \mathrm{~A}$ shows an example heatmap of the electrical activation on the MEA. The electrical signal travels from one or more pacemakers through the whole MEA surface in $9 \mathrm{~ms}$. The time and space differences were used in the velocity calculations. Under basal conditions the conduction velocity was reduced in TTS-iPSC-CMs $(4.97 \mathrm{~cm} / \mathrm{s})$ compared to CTRL-iPSC-CMs $(11.42 \mathrm{~cm} / \mathrm{s}$ ) (Figure 12B). Despite the different velocities, no obvious disturbances in conduction were observed (Figure 12B). When challenged with catecholamines, conduction velocity did not change significantly (Figure 12C/D/E/F). Interestingly, the observed basal differences remained as a trend throughout the catecholamine stimulation with Iso and with Epi (Figure 12C/D). 
A

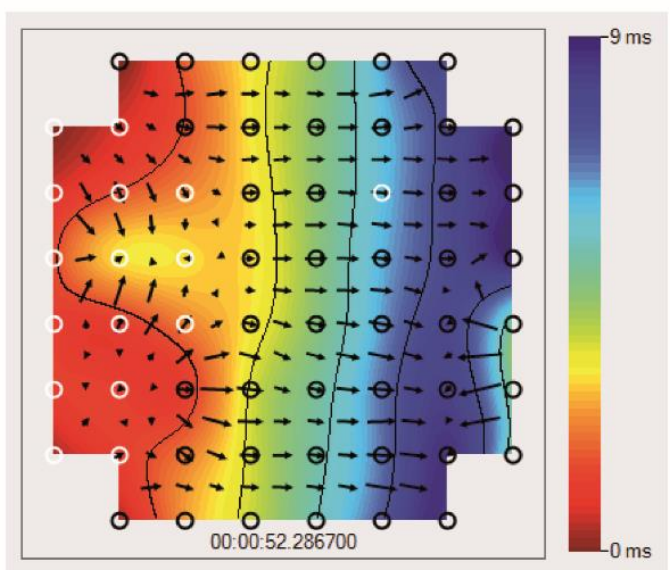

C

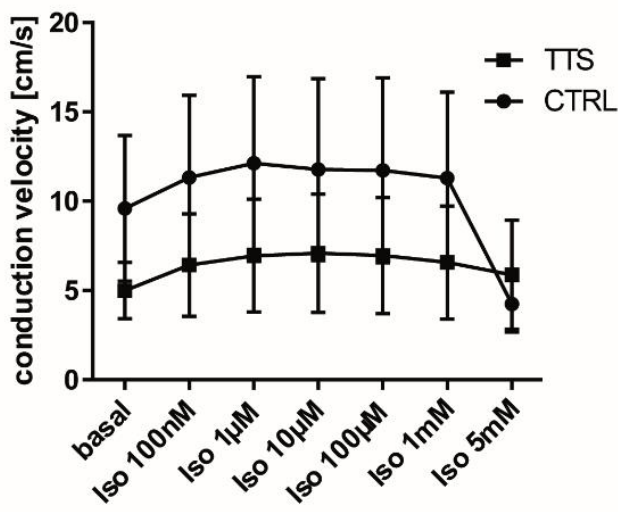

E

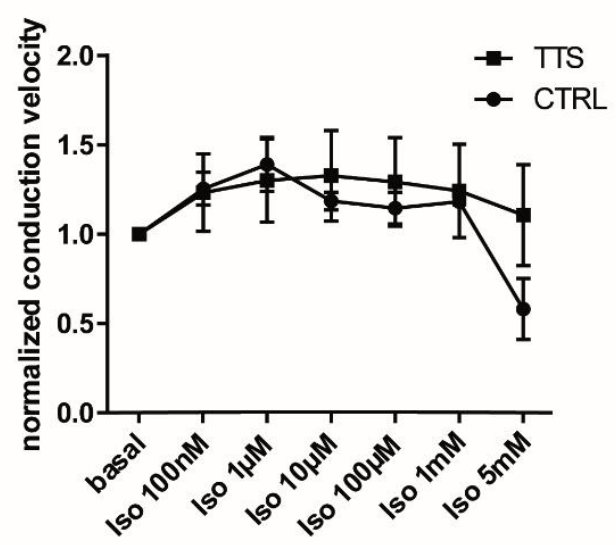

B

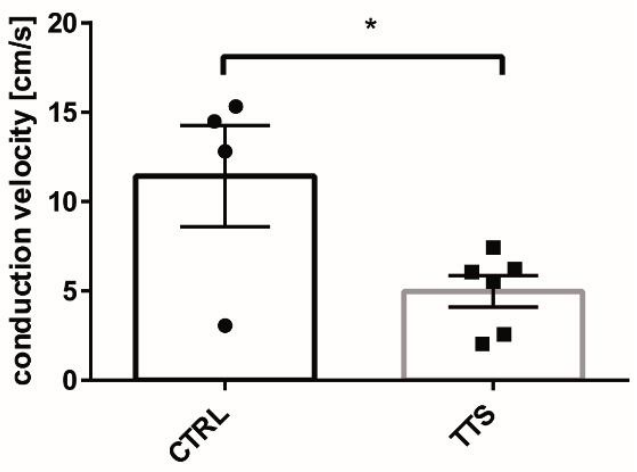

D

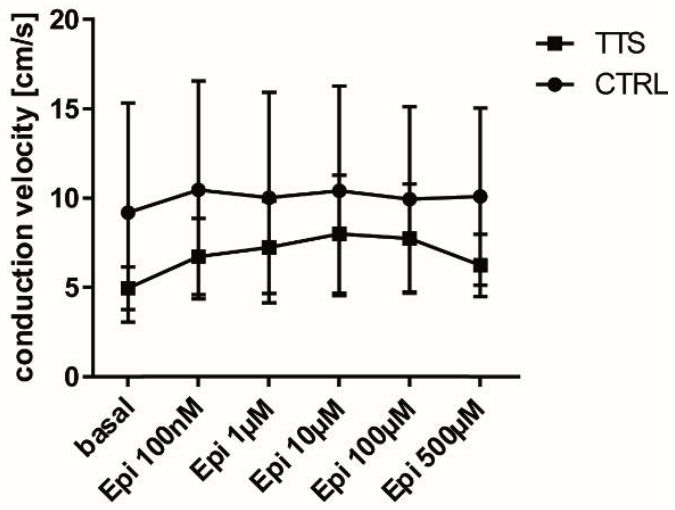

$\mathbf{F}$

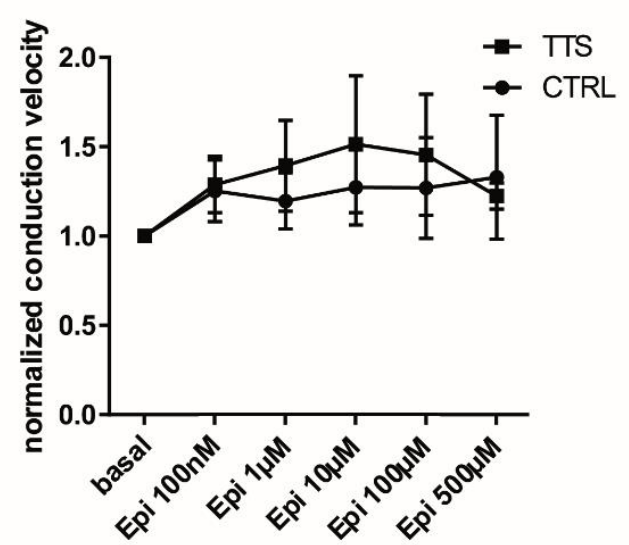

Figure 12: Measurements of conduction velocities with multi-electrode arrays. A: Representative conduction velocity map of a non-stimulated CTRL-iPSC-CM preparation. Colors indicate the timepoint of activation at a certain electrode (from red (early) to blue (late)). Lines represent zones, which are active at the same time. Arrows indicate the direction of the signal propagation. B: Basal conduction velocities of CTRL- ( $n=4$ MEA experiments: $2-C[n=4])$ and TTS-iPSC-CMs $(n=6$ MEA experiments: 5-TTS [ $n=4]$, and 8-TTS [ $n=2]$ ). CTRL-iPSC-CMs showed a significantly higher 
conduction velocity than TTS-iPSC-CMs. Significance was calculated using Student's $t$-test. C: Conduction velocities of Iso stimulated CTRL- ( $n=3$ MEA experiments: $2-C$ [ $n=3])$ and TTS-iPSCCMs ( $n=3$ MEA experiments: 5-TTS [n=2], and 8-TTS [ $n=1]$ ). D: Conduction velocities of Epi stimulated CTRL- ( $n=2$ MEA experiments: $2-C[n=2])$ and TTS-iPSC-CMs ( $n=3$ MEA experiments: 5-TTS [ $n=2]$, and 8-TTS [n=1]). E: Normalized conduction velocities of Iso stimulated CTRL- $(n=3$ MEA experiments: $2-C[n=3])$ and TTS-iPSC-CMs ( $n=3$ MEA experiments: 5 -TTS [ $n=2]$, and 8-TTS $[n=1]) . F$ : Normalized conduction velocities of Epi stimulated CTRL- $(n=2$ MEA experiments: 2-C [n=2]) and TTS-iPSC-CMs ( $n=3$ MEA experiments: 5-TTS [n=2], and 8-TTS [n=1]). Data are presented as mean \pm SEM. Sign Significances were calculated using unpaired Student's $t$-test (B) and two-way ANOVA (C-F).

\section{5 $\mathrm{Ca}^{2+}$ handling is different in TTS-iPSC-CMs}

To find out whether TTS-iPSC-CMs and CTRL-iPSC-CMs show differences in $\mathrm{Ca}^{2+}$ handling, $\mathrm{Ca}^{2+}$ imaging with fluo-4-am was performed. Figure 13 shows typical $\mathrm{Ca}^{2+}$ traces and fluorescence intensities of TTS- and CTRL-iPSC-CMs.

CTRL

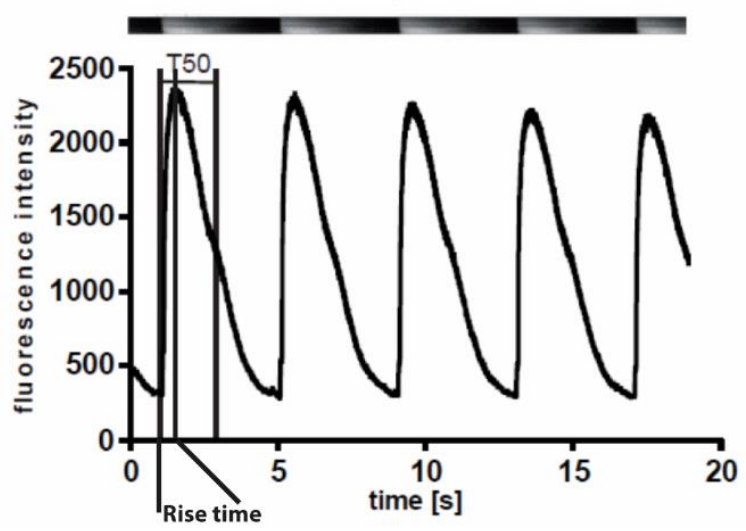

TTS

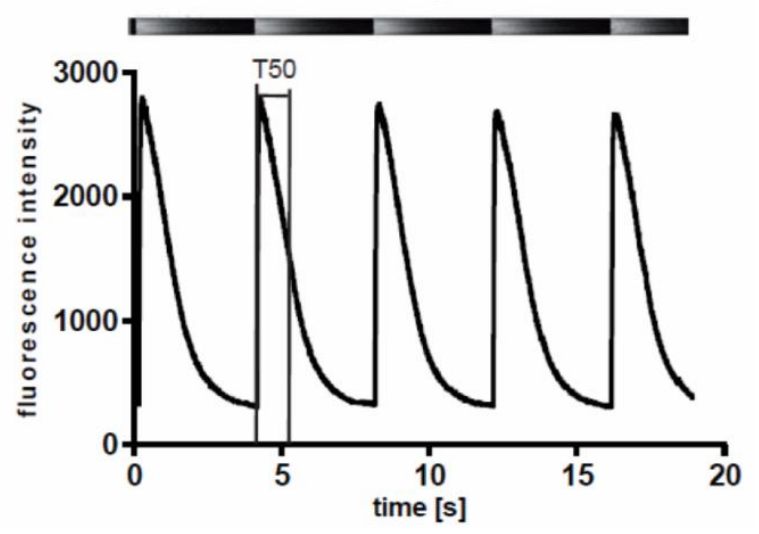

Figure 13: $\mathrm{Ca}^{2+}$ imaging recordings of CTRL-iPSC-CMs and TTS-iPSC-CMs. The upper part shows original line scans of single cells where the brightness corresponds to calcium $\left(\mathrm{Ca}^{2+}\right)$ concentrations. The lower part shows fluorescence intensities over time and the typical waves caused by calcium influx and efflux. $T_{50}$ marks the half-maximal decay time of the calcium transient.

$\mathrm{T}_{50}$ indicates the half-maximal decay time of the $\mathrm{Ca}^{2+}$ transient and is influenced mainly by the activity of the SERCA and NCX pumps. TTS-iPSC-CMs showed a significantly decreased $T_{50}$ time compared to CTRL-iPSC-CMs at basal levels, $100 \mathrm{nM}$ Iso, and $1 \mu \mathrm{M}$ 
Iso (Figure 14A). When normalized, the difference in T50 between CTRL- and TTS-iPSCCMs was lower because of the high basal differences (Figure 14B). TTS- and CTRL-iPSCCMs showed a significant decrease in $T_{50}$ times in response to rising Iso dosages (Figure 14B). TTS-iPSC-CMs showed a reduced $T_{50}$ time at $100 \mathrm{nM}$ Iso or higher, whereas CTRLiPSC-CMs needed $1 \mu \mathrm{M}$ Iso to achieve a significant reduction (Figure 14B). Interestingly, CTRL-iPSC-CMs and TTS-iPSC-CMs exhibit a reduction of $T_{50}$ even at high Iso dosages up to $5 \mathrm{mM}$ (Figure 14B). Supplemental Figure 1 shows normalized $T_{50}$ for individual patients uncovering that some probands reacted stronger (3-C, 8-TTS) towards Iso, while others reacted less (iFB2, 2-TTS). 

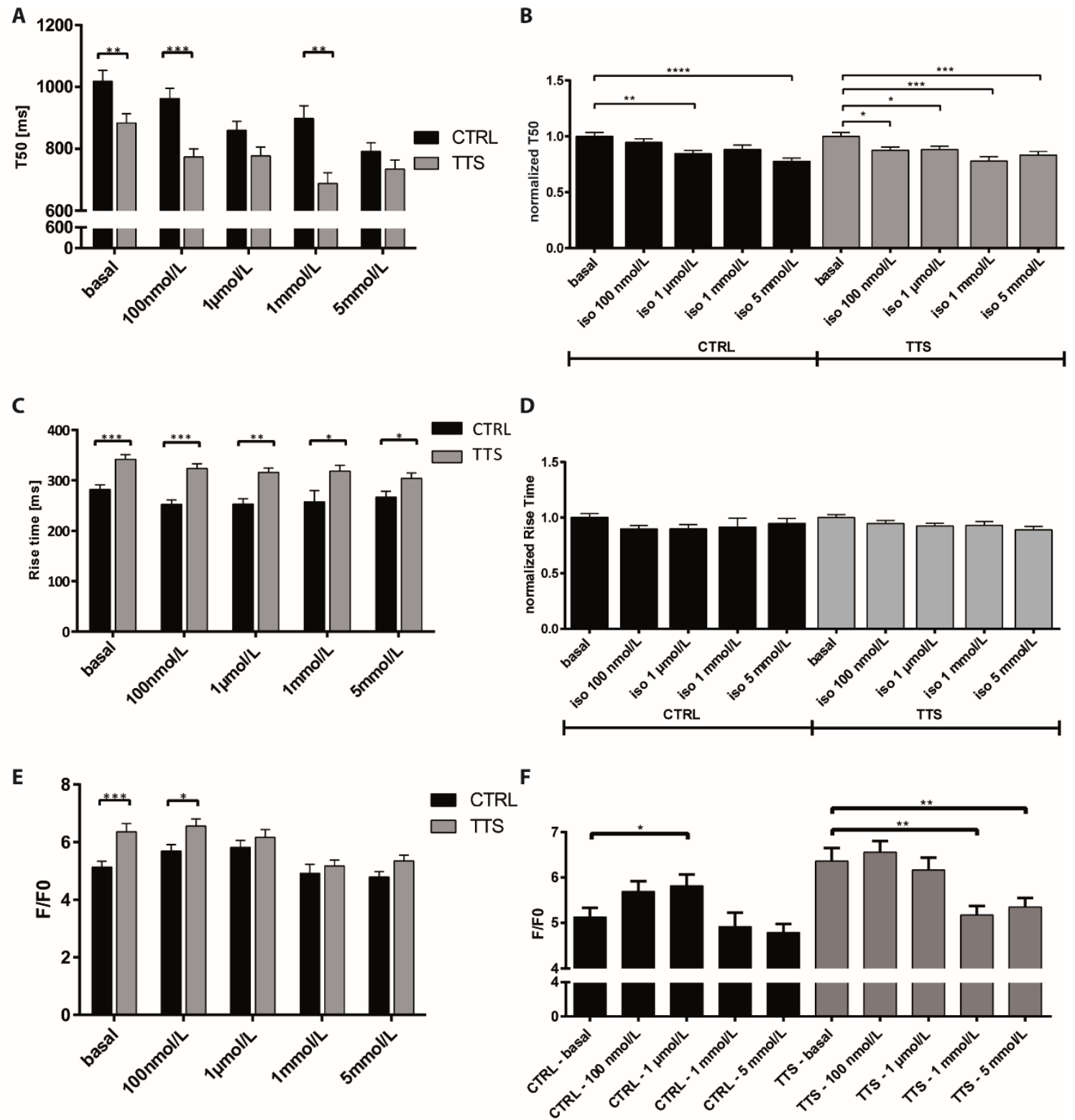

Figure 14: $\mathrm{Ca}^{2+}$ measurements of CTRL- and TTS-iPSC-CMs reveal $\mathrm{Ca}^{2+}$ handling differences. A: Analysis of half maximal decay times $\left(T_{50}\right)$ show a reduction in TTS-iPSC-CMs $(n=148$ cells: 2-TTS [ $n=26], 5$-TTS [ $n=60]$, and 8-TTS [ $n=62])$ compared to CTRL-iPSC-CMs $(n=153$ cells: 2-C [ $=47], 3-C$ [ $n=67]$, and iFB2 [ $n=39]$ ) at basal levels and after Iso addition. B: Normalized $T_{50}$ times in CTRL- $(n=153$ cells: $2-C$ [ $n=47], 3-C$ [ $\left.n=67\right]$, and iFB2 [ $\left.\left.n=39\right]\right)$ and TTS-iPSCCMs C: Rise time of the $\mathrm{Ca}^{2+}$ transient is increased in TTS-iPSC-CMs $(\mathrm{n}=148$ cells: 2 -TTS [ $\mathrm{n}=$ 26], 5-TTS [ $n=60$ ], and 8-TTS [ $n=62$ ]) compared to CTRL-iPSC-CMs ( $n=153$ cells: $2-C$ [ $n=$ 47], 3-C [ $n=67]$, and iFB2 [ $n=39])$. D: Normalized rise times of CTRL- and TTS-iPSC-CMs $(n=$ 148 cells: 2 -TTS $[n=26], 5$-TTS $[n=60]$, and 8-TTS $[n=62]) . E$, F: Transient height $(F / F 0)$ is increased in TTS-iPSC-CMs $(n=148$ cells: 2-TTS [ $n=26]$, 5-TTS $[n=60]$, and 8-TTS [ $n=62])$ in comparison with CTRL-iPSC-CMs ( $n=153$ cells: $2-C$ [ $n=47], 3-C$ [ $n=67]$, and iFB2 $[n=39])$. 
Data are presented as mean \pm SEM. Significances were calculated with two-way ANOVA (A, C, E) or one-way ANOVA (B, D, F).

Rise time specifies the time that is needed to reach the maximum $\mathrm{Ca}^{2+}$ concentration in a cardiomyocyte, which is mainly influenced by the activity of the L-type $\mathrm{Ca}^{2+}$ channel and the RyR2. TTS-iPSC-CMs exhibited a higher rise time compared to CTRL-iPSC-CMs under basal conditions (Figure 14C) and all tested Iso concentrations (Figure 14C). Interestingly, the addition of Iso to CTRL-iPSC-CMs and TTS-iPSC-CMs did not cause a significant decrease in rise time. However, a trend towards a reduction was observed for normalized rise times (Figure 14D).

$\mathrm{Ca}^{2+}$ transient amplitude (F/F0) provides information about the amount of $\mathrm{Ca}^{2+}$ influx in comparison to basal $\mathrm{Ca}^{2+}$ levels. TTS-iPSC-CMs exhibited a higher amplitude at basal levels and Iso 100 nM (Figure 14E, F). Interestingly, only CTRL-iPSC-CMs showed a robust increase in maximal amplitude when cells were stimulated with Iso. This effect was not seen in TTS-iPSC-CMs. CTRL- and TTS-iPSC-CMs showed a decrease in $\mathrm{Ca}^{2+}$ transient amplitude at higher Iso dosages.

\subsection{TTS-iPSC-CMs did not exhibit an increased $\mathrm{Ca}^{2+}$ leakage}

$\mathrm{Ca}^{2+}$ leakage is a hallmark of many cardiac diseases. Therefore $\mathrm{Ca}^{2+}$ sparks were analyzed. Figure 15A shows exemplified line scans of CTRL- and TTS-iPSC-CMs with $\mathrm{Ca}^{2+}$ sparks. Spark properties were analyzed for FWHM, FDHM, and Amplitude, which were used for total $\mathrm{Ca}^{2+}$ leak calculation (Figure 15B). For basal conditions and under Iso stimulation (100 nM), no differences in spark numbers were observed between CTRLand TTS-iPSC-CMs. CTRL-iPSC-CMs showed a higher spark frequency at Iso dosages of $1 \mu \mathrm{M}$ and $5 \mathrm{mM}$ Figure 15C. For FWHM (Figure 15D) and FDHM (Figure 15E), no significant differences were observed between CTRL- and TTS-iPSC-CMs. The overall total calcium leak did not show differences between CTRL- and TTS-iPSC-CMs for basal as well as for all tested Iso conditions (Figure 15F). In total, $\mathrm{Ca}^{2+}$ leakage differences were marginal. Supplemental Figure 3 shows the analyzed data for individual probands. 
A

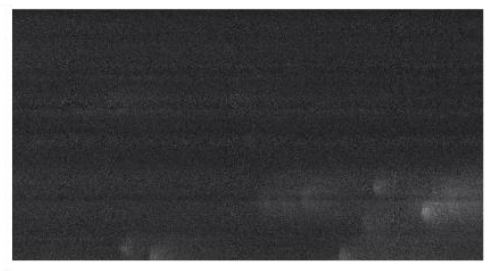

B

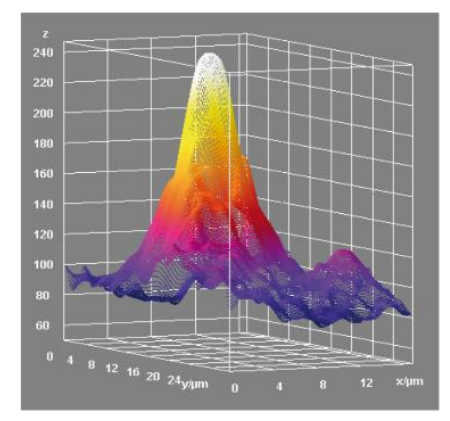

C

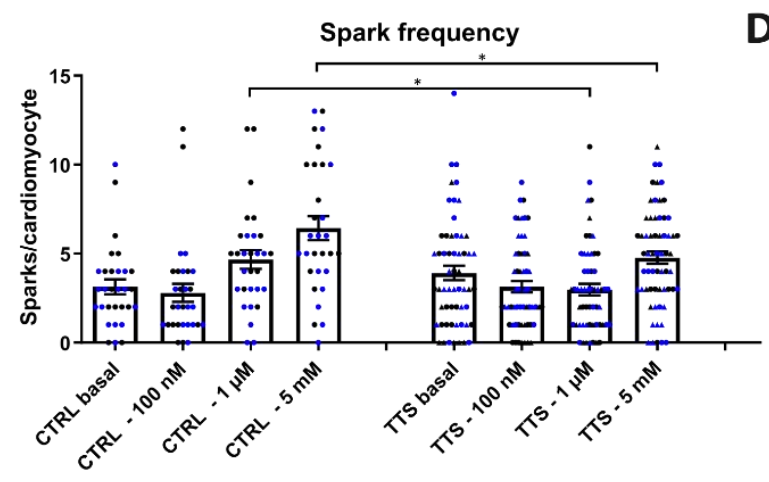

E

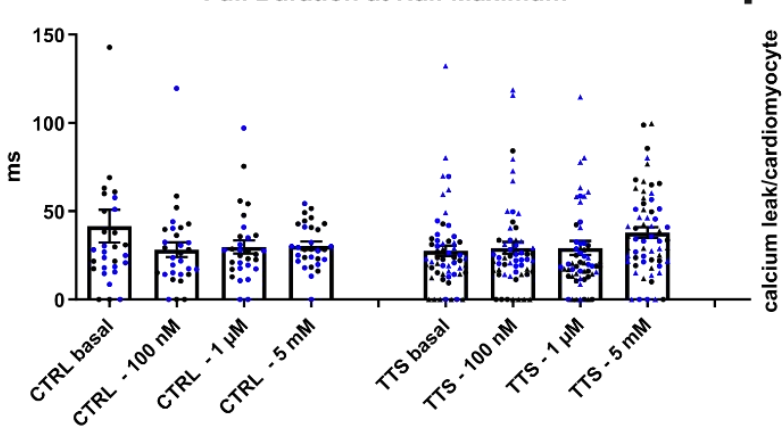

D

F
TTS
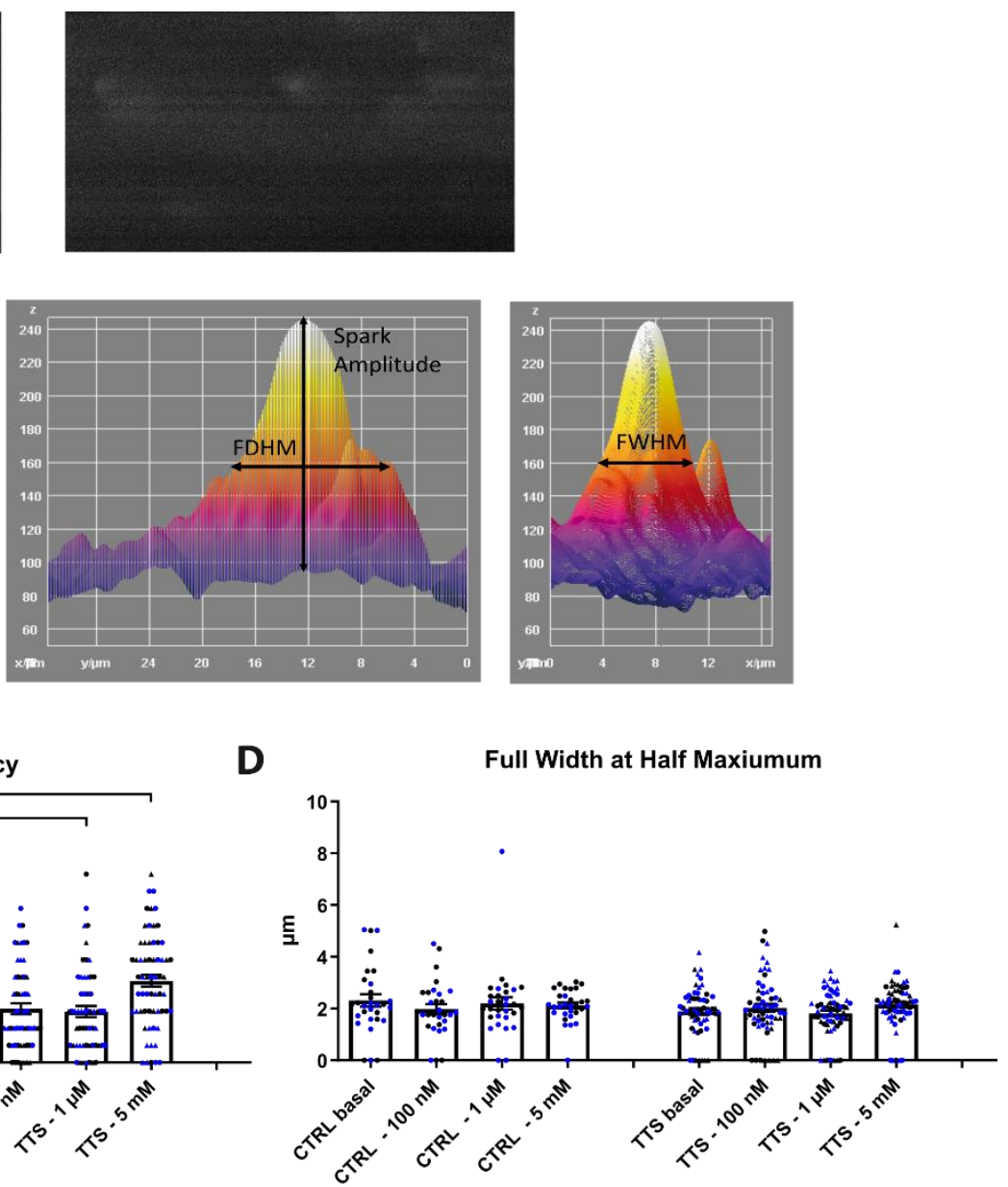

Total $\mathrm{Ca}^{2+}$ leak

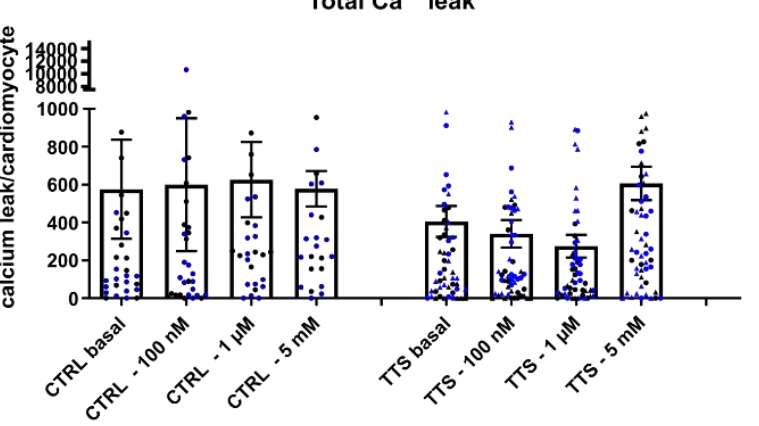

Figure 15: $\mathrm{Ca}^{2+}$ spark analysis. A: Example line scans with $\mathrm{Ca}^{2+}$ sparks of CTRL- and TTS-iPSCCMs. B: 3D rendering of a $\mathrm{Ca}^{2+}$ spark with the relevant size parameters. C: Spark frequency of CTRL- ( $n=30$ cells: $3-C$ [ $n=30$ cells, black and blue dots for 2 differentiations]) and TTS-iPSCCMs ( $n=57$ cells: 5-TTS [ $n=30$, black dots and blue dots for 2 differentiations], 8-TTS [ $n=27$, black triangles and blue triangles for 2 differentiations]) under Iso stimulation. D: Full width at half maximum (FWHM) of CTRL- $(n=30$ cells: $3-C$ [ $n=30$ cells, black and blue dots for 2 
differentiations]) and TTS-iPSC-CMs ( $n=57$ cells: 5 -TTS [ $n=30$, black dots and blue dots for 2 differentiations], 8-TTS [ $\mathrm{n}=27$, black triangles and blue triangles for 2 differentiations]) under Iso stimulation. E: Full duration at half maximum (FDHM) of CTRL- $(n=30$ cells: $3-C$ [ $n=30$ cells, black and blue dots for 2 differentiations]) and TTS-iPSC-CMs ( $n=57$ cells: 5 -TTS $[n=30$, black dots and blue dots for 2 differentiations], 8-TTS [ $n=27$, black triangles and blue triangles for 2 differentiations]) under Iso stimulation. F: Total calcium leak of CTRL- ( $n=30$ cells: $3-C$ [ $n=30$ cells, black and blue dots for 2 differentiations]) and TTS-iPSC-CMs ( $n=57$ cells: 5 -TTS $[n=30$, black dots and blue dots for 2 differentiations], 8-TTS [ $n=27$, black triangles and blue triangles for 2 differentiations]) under Iso stimulation. Data are presented as mean \pm SEM. Significances were calculated using two-way ANOVA.

\subsection{Engineered heart muscles reveal a higher sensitivity of TTS-iPSC-CMs towards Iso stimulation and decreased $\beta$-AR desensitization}

To obtain a clearer picture of the TTS model in a 3D environment, engineered heart muscles from TTS- and CTRL-iPSC-CMs were created and analyzed (cooperation with the Department of Pharmacology, University Medical Center Göttingen). The CMs were mixed with collagen and wildtype fibroblasts at a 70/30 ratio to create the engineered heart muscles (EHMs). EHMs were created and analyzed by Dr. Malte Tiburcy and Dr. Norman Liaw (Figure 16). When challenged with Iso, the TTS-iPSC-EHM showed a significantly higher sensitivity compared to CTRL-iPSC-EHM, as shown by a lower EC50 (6 $\pm 1 \mathrm{nM}$ vs. $13 \pm 2 \mathrm{nM}$ ) (Figure 16A). Blockage of the $\beta_{1}$ - and $\beta_{2}-A R s$ with CGP and ICI increased the $\mathrm{EC}_{50}$ values and removed all $\mathrm{EC}_{50}$ differences (Figure 16A). Additionally, beating frequency changes after $24 \mathrm{~h}$ of Iso stimulation were higher in TTS-iPSC-EHM compared to CTRL-iPSC-EHM (Figure 16B) $(222 \% \pm 32 \%$ vs. $59 \% \pm 19 \%$ ), which is in line with the reported frequency differences in the MEA measurements. After $24 \mathrm{~h}$ of Iso prestimulation, the observed $\mathrm{EC}_{50}$ differences were even higher than before as a sign of solid $\beta-A R$ desensitization with CTRL-iPSC-EHM (EC50: $64 \pm 10 \mathrm{nM})$ exhibiting a stronger $\mathrm{EC}_{50}$ increase than TTS-iPSC-EHM (EC50: $19 \pm 4$ nM) (Figure 16C). Interestingly, TTS-iPSCEHM showed a lower force development than CTRL-iPSC-EHM without catecholamine stimulation (Figure 16D). 
A

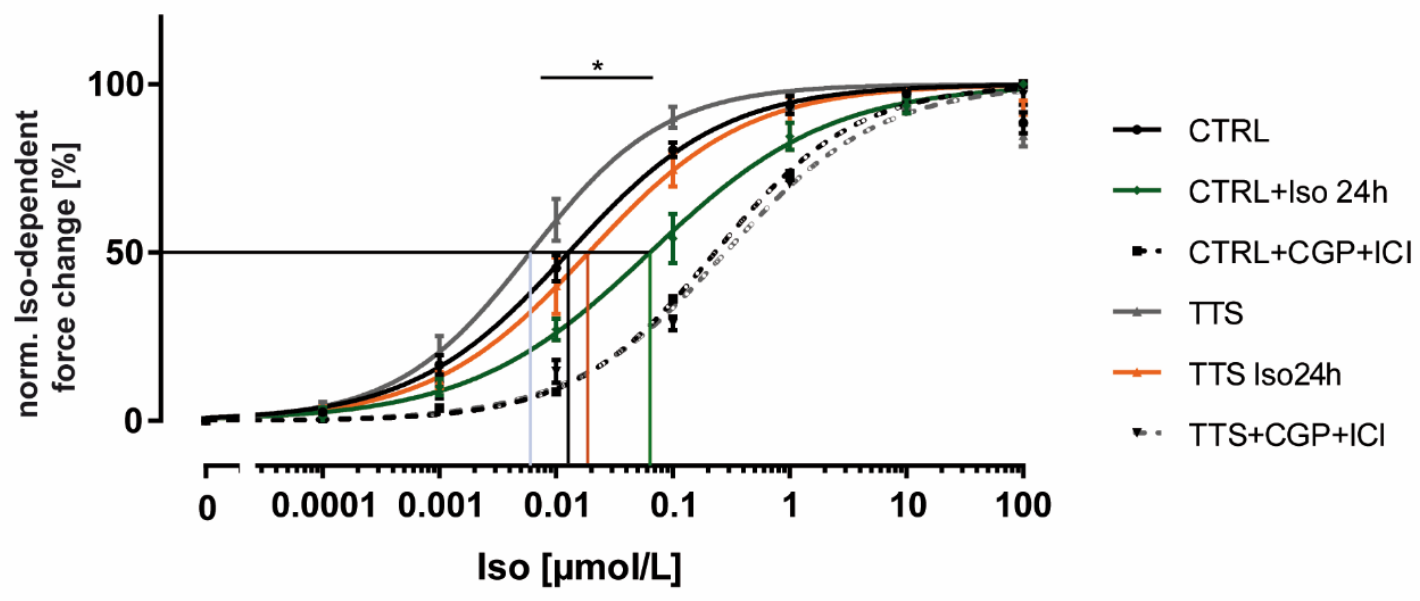

B

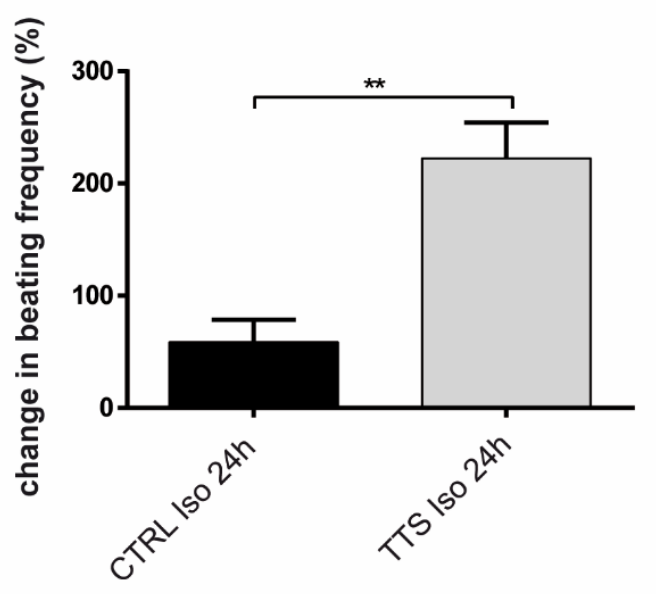

C

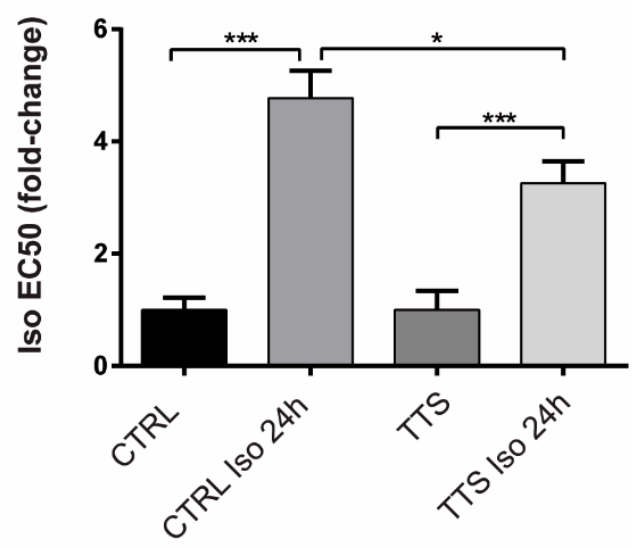

D

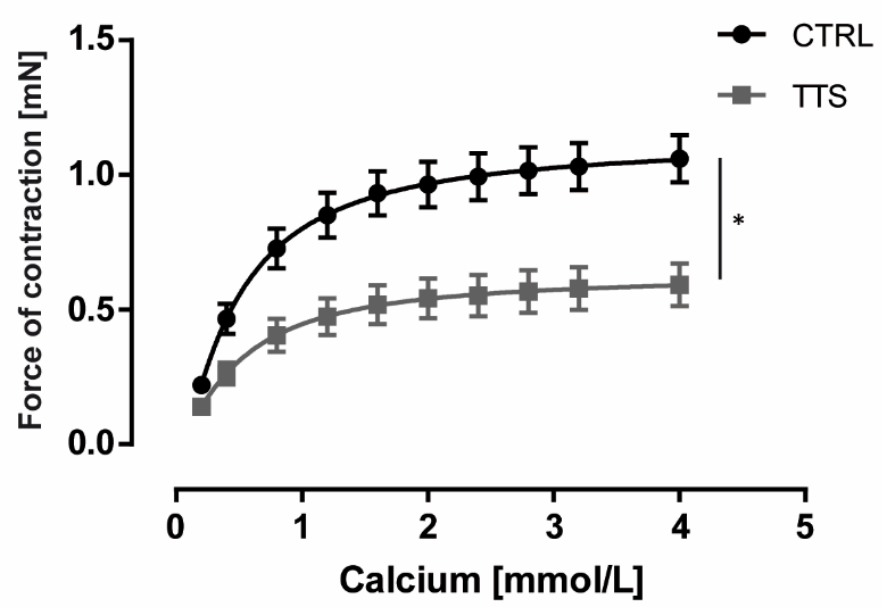

Figure 16: Engineered heart muscles (EHM) uncover an enhanced sensitivity towards Iso of TTSiPSC-CMs. A: Dose-response curves of CTRL- and TTS-iPSC-EHM towards increasing dosages of Iso. TTS-iPSC-EHM (grey line, $n=10$ EHM: 1-TTS [ $n=3]$, 8-TTS [ $n=7]$ ) exhibited a lower $E_{50}$ concentration than CTRL-iPSC-EHM (black line, $n=11$ EHM: 2-C [ $n=4$ EHM], iFB2 [ $n=7]$ ). After 24 $h$ Iso pre-stimulation, TTS-iPSC-EHM (orange line, $n=7$ EHM: 1 -TTS [ $n=3$ ], and 8-TTS [ $n=4$ ]) again showed a lower $\mathrm{EC}_{50}$ concentration than CTRL-iPSC-EHM (green line, $n=5$ EHM: 2-C [ $\left.n=2\right]$, and 
iFB2 [ $n=3]$ ). Addition of CGP and ICl (black dashed lines=CTRL, $n=4$ EHM: iFB2 [n=4], grey dashed lines=TTS, $n=4$ EHM: 8-TTS $[n=4]$ ) blocked all $E_{50}$ differences. $B$ : Change in beating frequency of EHM after $24 \mathrm{~h}$ Iso stimulation. TTS-iPSC-EHM ( $n=7$ EHM: 1-TTS [ $n=3$ ], and 8-TTS $[n=4])$ show a higher increase in beating frequency than CTRL-iPSC-EHM ( $n=5$ EHM: 2-C [ $n=2]$, and iFB2 [n=3]). C: $\mathrm{EC}_{50}$ increase after Iso preincubation $(1 \mu \mathrm{M})$ for $24 \mathrm{~h}$. CTRL-iPSC-EHM (EHM from Figure $16 \mathrm{~A}$ were analyzed) showed a stronger increase of $\mathrm{EC}_{50}$ after $24 \mathrm{~h}$ Iso preincubation than TTS-iPSC-EHM (EHM from Figure 16A were analyzed). D: Force development of EHMs at different $\mathrm{Ca}^{2+}$ concentrations. TTS-iPSC-EHM ( $n=14$ EHM: 1-TTS [ $\left.n=3\right], 8-T T S$ [ $\left.\left.n=11\right]\right)$ showed a lower force development than CTRL-iPSC-EHM ( $n=15$ EHM: 2-C [ $n=4]$, and iFB2 [ $n=11])$. Data are presented as mean \pm SEM. Differences were compared by extra sum-of-squares $F$ test $(A)$, Student's $t$-test (B), one-way ANOVA (C), or two-way ANOVA (D).

\subsection{TTS cells demonstrated lipid accumulation}

Cardiac adrenergic stimulation regulates lipid metabolism by influencing the PKA pathway and NR4A1 induction (Maxwell et al., 2005; Shao et al., 2013). Additionally, experiments in our group uncovered a higher NR4A1 increase in TTS-iPSC-CMs in comparison to the CTRL-iPSC-CMs when stimulated with Iso (Borchert et al., 2017). Therefore, lipid accumulation was analyzed in catecholamine-treated iPSC-CMs by Oil Red O staining. Lipid staining revealed only minor lipid accumulation in untreated cells. After Epi addition, lipid droplets were increased in TTS-iPSC-CMs. No lipid accumulation was observed in CTRL-iPSC-CMs (Figure 17A/B, lipid accumulations are indicated by white arrows). The droplet amount did increase 5.0 to 5.7 -fold for $100 \mathrm{nM}$ or $500 \mu \mathrm{M}$ Epi, respectively (Figure 17B). Interestingly, no significant lipid droplet increase was observed in Iso-treated iPSCCMs (Figure 17B).

Based on the obtained data, the expression of different lipid transporters was analyzed. The lipid importer CD36 expression was significantly increased on mRNA level (Figure 17C) in TTS-iPSC-CMs already at basal levels and under Epi $500 \mu \mathrm{M}$ stimulation arguing for a higher lipid import into these cells. During Epi stimulation, no CD36 expression changes were observed in CTRL- and TTS-iPSC-CMs. In line with the observed lipid accumulation, there was a decreased mRNA expression of the lipid translocase CPT1C in TTS-iPSC-CMs compared to CTRL-iPSC-CMs for Iso 100 nM, Epi 100 nM, and Epi 500 $\mu \mathrm{M}$ (Figure 17D). 

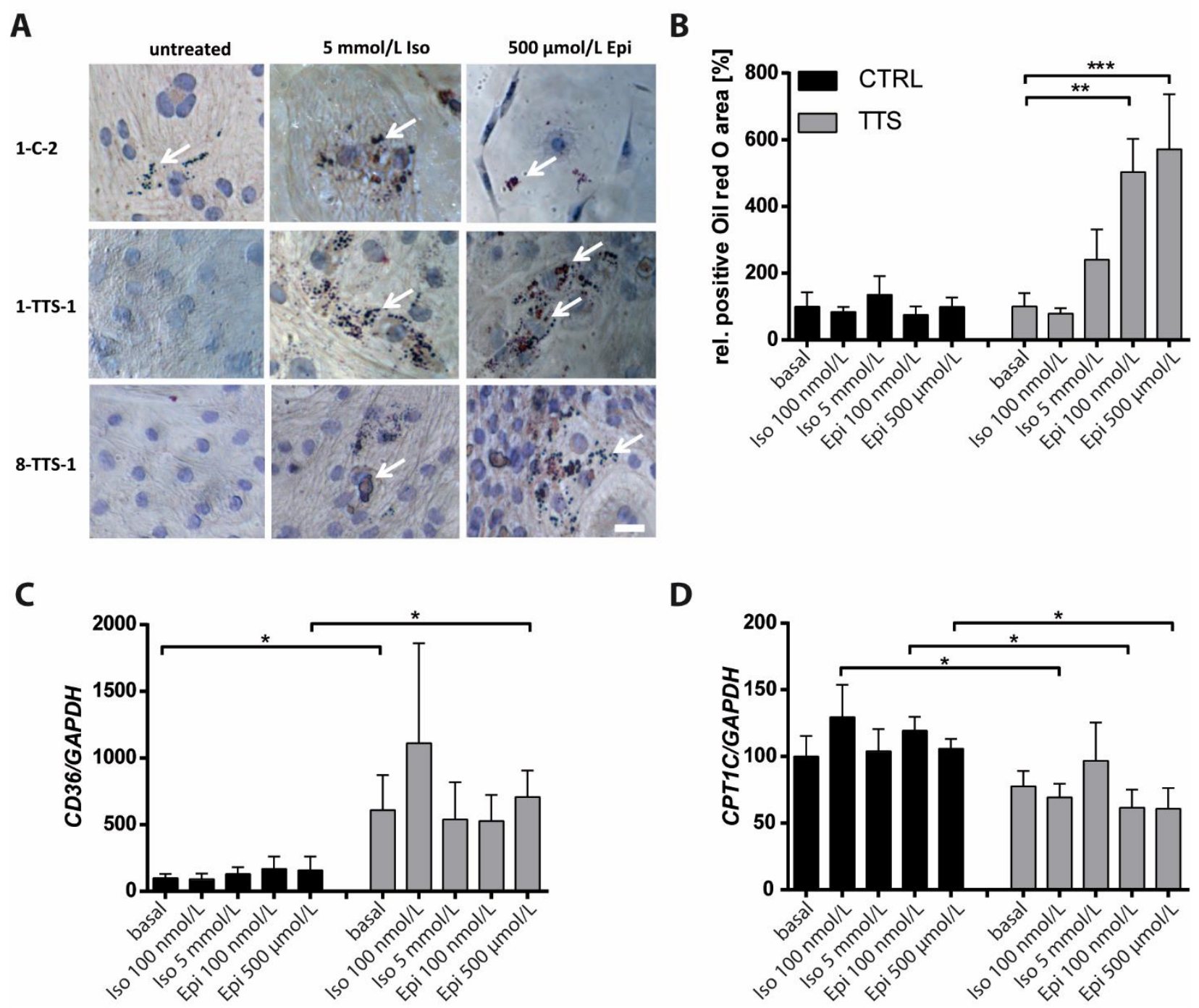

Figure 17: TTS-iPSC-CMs show lipid accumulation and differentially expressed lipid transporters. A: Lipid accumulation after catecholamine stimulation for $2 \mathrm{~h}$ in CTRL- and TTS-iPSC-CMs was visualized by Oil Red O staining. Lipid droplets are depicted by arrows. B: Quantification of the Oil Red O lipid accumulation. TTS-iPSC-CMs ( $n=4$ to 14 images: 1 -TTS [ $n=3$ to 9], 8-TTS [ $n=1$ to 7]) showed a prominent increase under Epi stimulation and a moderate increase after Iso stimulation compared to CTRL-iPSC-CMs ( $n=3$ to 9 images: $1-C$ [ $n=2$ to 8], iFB2 [ $n=3]$ ). Differences between the groups were compared using one-way ANOVA. C, D: mRNA expression of the lipid importer CD36 was increased and expression of the lipid translocator CPT1C was decreased in TTS-iPSC-CMs ( $n=7$ differentiation experiments: 1 -TTS [ $n=3]$ and 8-TTS [ $n=4]$ ) compared to CTRL-iPSC-CMs ( $n=7$ differentiation experiments: $1-C$ [ $n=3], 2-C$ [ $n=2$ ], and iFB2 $[n=2]$. Significant differences were calculated by one-way ANOVA (B) and Student's $t$-test (C, D). 


\subsection{Inhibition of selected $\beta$-pathways uncovers the role of different $\beta$-AR sub-receptors}

To dissect the influence of the different $\beta$-ARs in cardiomyocytes, the $\beta_{1}$-specific inhibitor CGP was used at a concentration of $100 \mathrm{nM}$ and the $\beta_{2}$-specific inhibitor ICI was used at a concentration of $50 \mathrm{nM}$ in combination with catecholamine stimulation. As the heart mainly expresses $\beta_{1}$ - and $\beta_{2}-A R s$, blockage of one of these receptors uncovers the influence of the other. A combination of CGP and ICI was used to assess the behavior of the two receptors, when both dominant $\beta$-ARs were silenced.

\subsubsection{MEA measurements emphasize the roles of different $\beta$-ARs in TTS-iPSC-CMs}

MEAs were used to analyze the inherent beating frequency of the CTRL-iPSC-CMs and TTS-iPSC-CMs under the influence of the different $\beta$-AR subtype-specific $\beta$-blockers in combination with increasing levels of catecholamines.

CTRL-iPSC-CMs and TTS-iPSC-CMs exhibited increasing beating frequencies under Iso treatment, as shown before (Figure 10, Figure 18A-D). In CTRL-iPSC-CMs, a combination of CGP and ICl was able to significantly suppress the beating frequency increase at Iso concentrations of $100 \mathrm{nM}$ and $1 \mu \mathrm{M}$ in comparison to Iso-stimulated CTRL-iPSC-CMs without $\beta$-AR inhibitors (Figure $18 \mathrm{~A} / \mathrm{C}$, purple line). Higher Iso concentrations caused a frequency increase. At Iso concentrations of $1 \mathrm{mM}$, frequencies of CTRL-iPSC-CMs of all conditions reached the same level (Figure 18A/C). Overall, CTRL-iPSC-CMs treated with CGP showed no significant differences in frequency upregulation in response towards Iso in comparison to cells not treated with $\beta$-AR-blockers (Figure 18A/C, blue line). In contrast, when CTRL-iPSC-CMs were treated with $\mathrm{ICl}$, the frequency upregulation was significantly lower at $100 \mathrm{nM}$ Iso compared to untreated cells (Figure 18A/C, red line). These findings suggest an important role of the $\beta_{2}$ - $A R$ in controlling the chronotropic response in CTRLiPSC-CMs. 
A

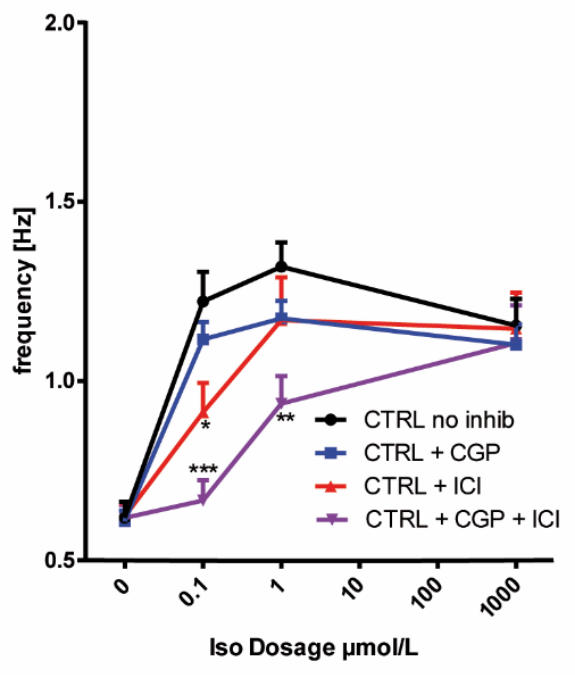

C

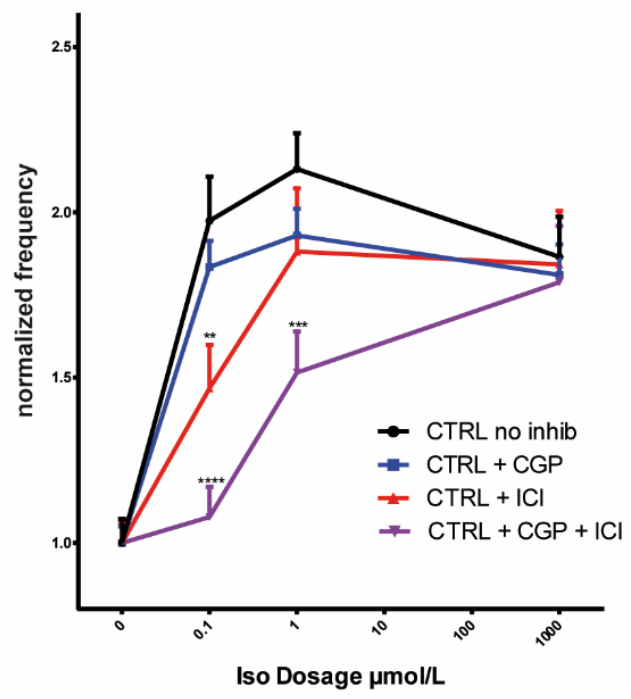

B

TTS

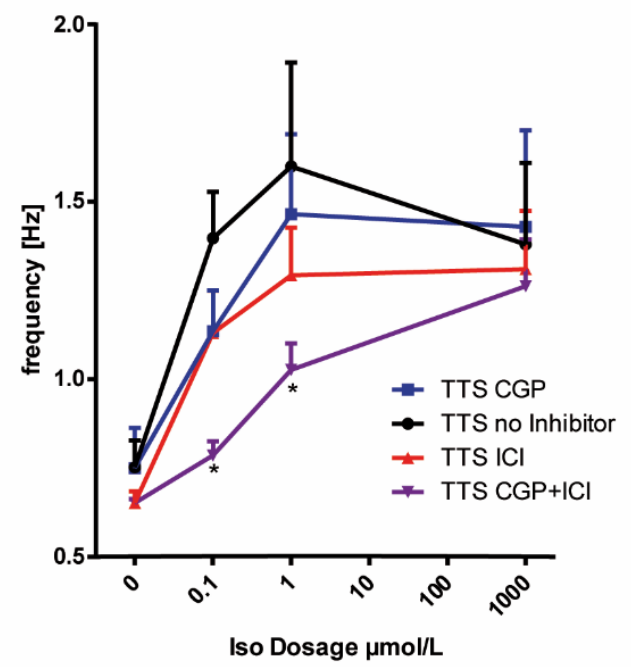

D

TTS

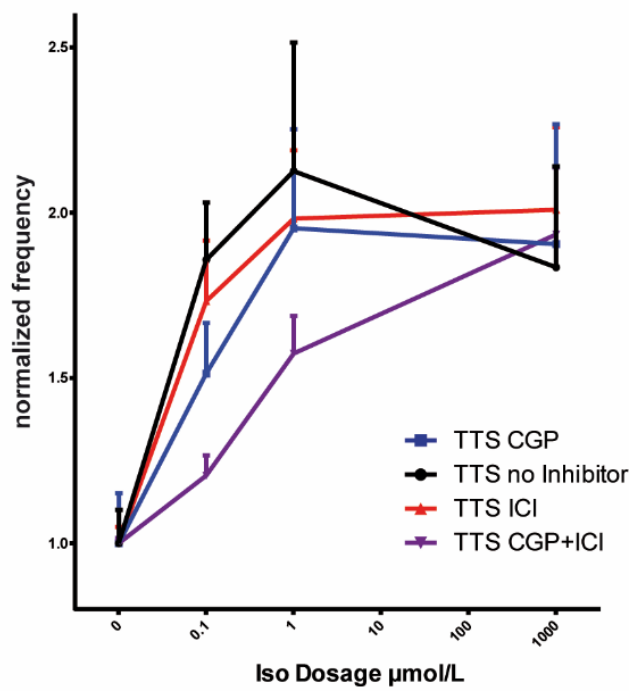

Figure 18: Influence of selective $\beta$-inhibition on electrical behavior of CTRL- and TTS-iPSC-CMs. A, B: Iso dose-dependent frequency increase under influence of CGP, ICI or CGP+ICI for CTRLiPSC-CMs ( $n=3$ MEA experiments: $2-C$ [n=2], iFB2 [n=1]) vs TTS-iPSC-CMs ( $n=3$ MEA experiments: 2-TTS [n=1], 5-TTS [n=1], 8-TTS [n=1]). C, D: Normalized Iso dependent frequency increase under influence of CGP, ICl, or a combination thereof. In CTRL-iPSC-CMs (same MEA experiments as in $A / B), \beta_{2}$-inhibition by $I C l$ caused a significant decrease in the beating rate. In TTS-iPSC-CMs (same MEA experiments as in A/B), both $\beta$-ARs seem to play a significant role. The use of both inhibitors caused a strong decrease in the frequency compared to no inhibitor as expected. Data are presented as mean \pm SEM. Differences between each $\beta$-inhibitor combination at the same Iso level were compared using two-way ANOVA. 
In TTS-iPSC-CMs, only the combination of CGP and ICI produced a significant effect in a similar way to CTRL-iPSC-CMs, resulting in a lower frequency for this treatment compared with TTS-iPSC-CMs treated only with Iso (Figure 18B/D). CGP or ICI treatment alone was not able to produce a significant effect. A trend towards lower frequencies, however, was visible (Figure 18B/D). This finding suggests that both $\beta$-ARs are important in TTS-iPSCCMs.

\subsection{2 $\mathrm{Ca}^{2+}$ measurements support the dominant role of $\beta_{2}$-ARs in CTRL-iPSC-CMs and $\beta_{1}$-ARs in TTS-iPSC-CMs}

To check whether the different $\beta$-AR subtypes also influence $\mathrm{Ca}^{2+}$ signaling, $\mathrm{CGP}$ and $\mathrm{ICI}$ were used as described above. Additionally, Iso was used to stimulate the cardiomyocytes and $\mathrm{Ca}^{2+}$ cycling was measured and normalized $\mathrm{T}_{50}$ and rise times were analyzed.

Without inhibitors, $T_{50}$ times were reduced as described (Figure 14A). Blockage of both $\beta$ ARs was able to block a $T_{50}$ reduction for Iso dosages below $1 \mathrm{mM}$ for CTRL- and TTSiPSC-CMs (Figure 19A). Without Iso stimulation, the addition of CGP and ICI caused a reduction of $T_{50}$ times in CTRL-iPSC-CMs (Supplemental Figure 2). Relative $T_{50}$ analysis uncovered that CGP-treated CTRL-iPSC-CMs showed a significantly reduced $T_{50}$ time at Iso concentrations of $1 \mu \mathrm{M}$, whereas CTRL-iPSC-CMs treatment with ICI showed the same effect for much higher Iso concentrations (5 mM) (Figure 19A). As expected, the addition of CGP and ICI suppressed all $T_{50}$ reductions caused by Iso in CTRL-iPSC-CMs (Figure 19A).

In TTS-iPSC-CMs, at basal conditions, blockage of the $\beta_{2}-\mathrm{AR}$ with ICl caused an increase in $T_{50}$ (Supplemental Figure 2). Treatment with the $\beta_{1}$-AR antagonist CGP abolished all $T_{50}$ reductions caused by Iso treatment of all concentrations (Figure 19A). When treated with the $\beta_{2}-\mathrm{AR}$ antagonist $\mathrm{ICl}, 100 \mathrm{nM}$ Iso was not enough to cause a significant decrease of $\mathrm{T}_{50}$. However, stimulation with higher Iso dosages from $1 \mu \mathrm{M}, 1 \mathrm{mM}$, and $5 \mathrm{mM}$ resulted in a significant decrease in relative $T_{50}$ times. CGP and ICI together were able to suppress an Iso-dependent reduction in $T_{50}$ times for Iso dosages lower than $1 \mathrm{mM}$ (Figure 19A). 
The addition of CGP or ICI without Iso caused an increase in rise time in CTRL-iPSC-CMs (Supplemental Figure 2). When challenged with CGP, CTRL-iPSC-CMs showed a reduced rise time under Iso stimulation from $1 \mathrm{mM}$ to $5 \mathrm{mM}$ (Figure 19B). No rise time reduction was observed when cells were challenged with ICI and Iso (Figure 19B). When CTRL-iPSC-CMs were treated with CGP and ICI together, different Iso dosages were not able to significantly reduce the rise time (Figure 19B). Without Iso stimulation, the addition of CGP caused a decrease of rise time in TTS-iPSC-CMs and the addition of ICI caused an increase in rise time (Supplemental Figure 2). Blockage of the $\beta_{1}$-AR with CGP and a subsequent challenge of Iso did not cause a reduction of the rise time in TTS-iPSC-CMs (Figure 19B). In contrast, the addition of ICI and Iso to TTS-iPSC-CMs caused a reduction of rise time at Iso concentrations from $100 \mathrm{nM}$ to $5 \mathrm{mM}$ (Figure 19B). Co-stimulation with CGP and ICl, together with Iso, did not result in a reduction of rise time (Figure 19B).

In conclusion, these findings further support the hypothesis that $\beta_{2}-A R$ plays an important role in CTRL-iPSC-CMs. Furthermore, they strengthen the additional important role of the $\beta_{1}$-ARs in TTS-iPSC-CMs. However, they also show that both $\beta$-ARs contribute to full activation in the measured cardiomyocytes, especially in TTS-iPSC-CMs. 
A
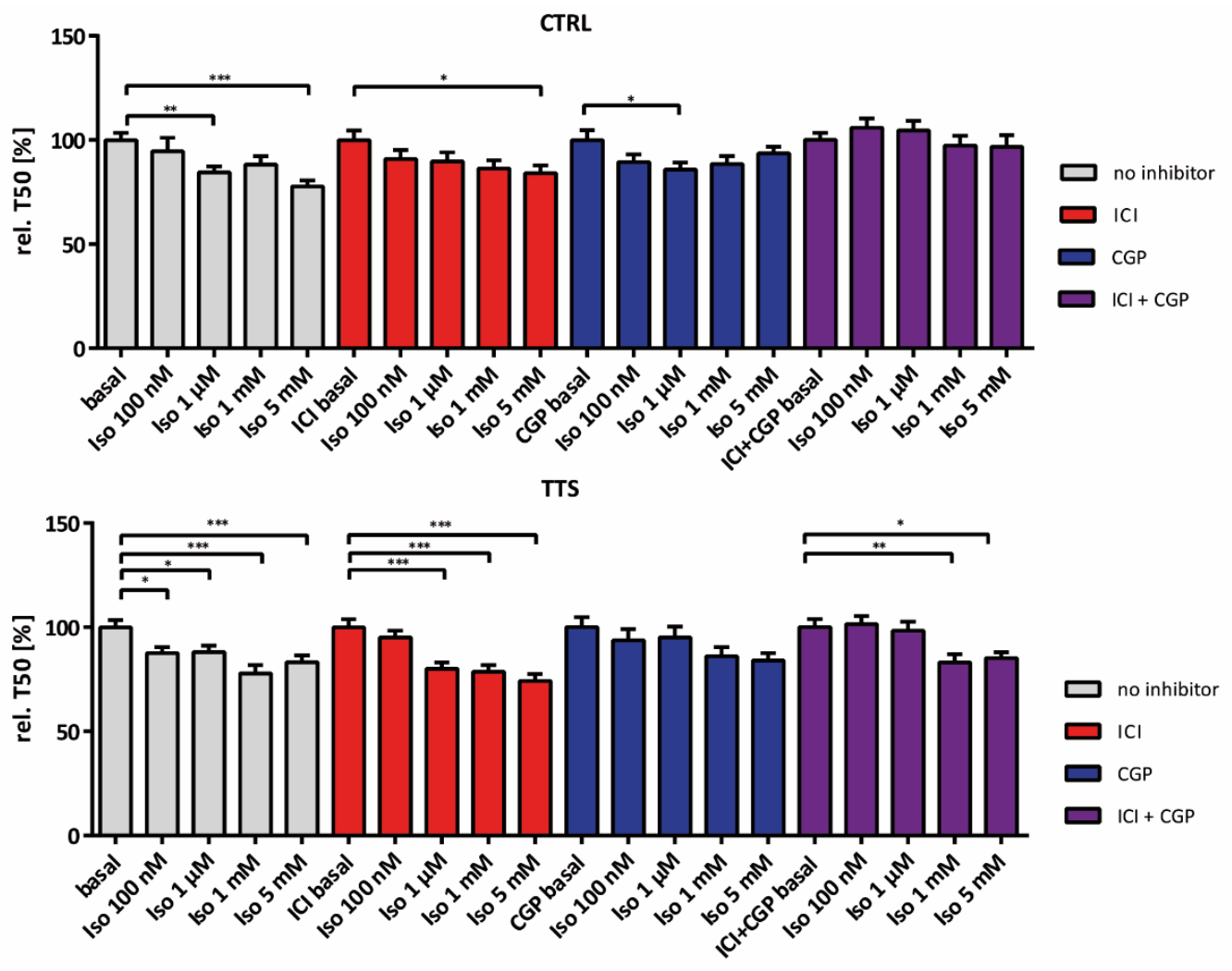

B
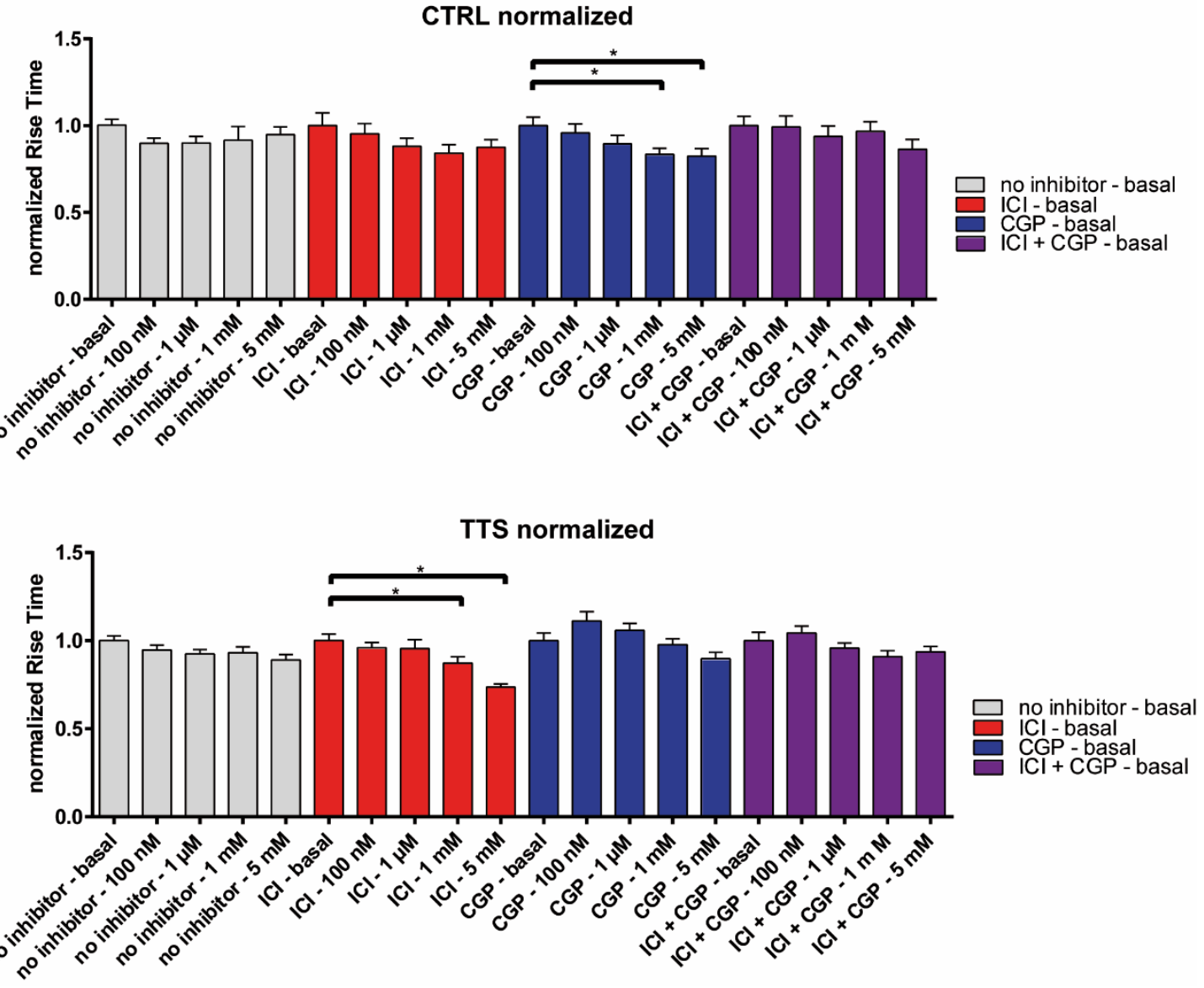
Figure 19: Selective $\beta$-inhibition with CGP and ICI uncover different roles of $\beta_{1}$ - and $\beta_{2}$-ARs in CTRL- and TTS-iPSC-CMs. A: Normalized $T_{50}$ times of CTRL- $(69$ cells: $2-C$ [ $n=25], 3-C$ [ $\left.n=22\right]$, iFB2 [ $n=22]$ ) and TTS-iPSC-CMs (73 cells: 2-TTS [ $n=26]$, 5-TTS [ $n=24], 8-T T S$ [ $n=23]$ ) treated with CGP, ICI or CGP + ICl in combination with Iso. $\beta_{2}$-ARs seem to play an important role in CTRL-iPSC-CMs and $\beta_{1}$-ARs in TTS-iPSC-CMs. Data are presented as mean \pm SEM and compared using one-way ANOVA. B: Normalized rise time measurements with CGP, ICl, or CGP $+\mathrm{ICl}$ in combination with Iso. Again, $\beta_{2}$-ARs were engaged in response to Iso stimulation in CTRLiPSC-CMs (same cells as in A), whereas $\beta_{1}$-ARs are important in TTS-iPSC-CMs (same cells as in A). Addition of CGP and ICI blocked all rise time reductions caused by Iso. Data are presented as mean \pm SEM. Differences were compared using one-way ANOVA. 


\section{Discussion}

Takotsubo syndrome is a severe heart disease triggered by excessive emotional or physical stress. These high-stress levels cause the release of high concentrations of catecholamines in the circulation, which may result in partial heart failure by left ventricular apical dyskinesia and basal hyperkinesia. Symptoms resulting from TTS are similar to those in patients with MI. It is commonly treated with a wide range of drugs like ACEinhibitors, $\beta$-blockers, heparin, and ASS in combination with intensive care (Ghadri et al., 2018b; Templin et al., 2015). Interestingly, after surviving the initial event, heart function recovers over the course of days to weeks. Unfortunately, a TTS attack increases the risk for future adverse cardiovascular diseases like heart attack and stroke. In consequence, the 5-year survival rate of TTS is similar to that of an acute STEMI (Templin et al., 2015). Thus, it is of utmost importance to learn more about this cryptic disease.

Not much is known about the causes and the pathomechanisms of TTS. However, with some hints towards a genetic component, modeling of Takotsubo syndrome with iPSCCMs could shed light on the pathophysiology of the disease. This work demonstrates the feasibility of a human TTS model based on iPSC-CMs to experimentally study TTS using cell culture techniques.

To this end, fibroblasts or PBMCs from four different TTS patients and three control probands were successfully reprogrammed to iPSCs using integration-free methods including Sendai viruses or gene transfection with plasmids. The resulting iPSCs expressed typical pluripotency-related genes at mRNA and protein level. The cells could be readily differentiated into cells from all three different germ-layers in vitro expressing germ-layer-specific genes at both mRNA and protein levels. Furthermore, the generated iPSCs differentiated in vivo in the form of teratomas showing properties of endodermal, mesodermal, and ectodermal tissues. In summary, the produced iPSCs showed the same attributes as hESCs and can be considered pluripotent.

The iPSCs were then differentiated into iPSC-CMs with the help of Wnt-pathway manipulation using the small molecules IWP2 and the GSK-inhibitor CHIR. The iPSC- 
CMs showed a purity of more than $80 \%$ to $95 \%$, as measured by flow cytometry with antibodies against cTnT. The produced iPSC-CMs showed spontaneous beating activity, expressed cardiomyocyte-specific genes ACTN2, MYH6, MYH7, and TNNT2 on mRNA and $\mathrm{cTnT}$ and $\alpha$-actinin on protein level. No difference in sarcomeric structure organization was observed. Furthermore, the CTRL- and TTS-iPSC-CMs showed electrical coupling and coordinated beating activity. Additionally, the cells could be paced electrically and showed regular $\mathrm{Ca}^{2+}$ waves typical for cardiomyocytes.

Treatment of iPSC-CMs with increasing catecholamine levels was carried out to mimic the TTS situation and analyzed for apoptosis, electrical activity $\mathrm{Ca}^{2+}$ homeostasis, behavior in a 3D-EHM system, and lipid accumulation. High Iso treatment did not induce significant levels of apoptosis. When stimulated with rising levels of Iso, CTRL-iPSC-CMs and TTS-iPSC-CMs stopped spontaneous electrical activity, with TTS-iPSC-CMs being more prone towards this effect. Additionally, TTS-iPSC-CMs showed a stronger frequency increase upon Iso stimulation. The conduction velocity was reduced in TTS iPSC-CMs in comparison to CTRL-iPSC-CMs. $\mathrm{Ca}^{2+}$ handling differed between TTS-iPSC-CMs and CTRL-iPSC-CMs were present on multiple levels. Under basal conditions, times to $50 \%$ decay were shorter, and rise times were longer in TTS-iPSC-CMs in comparison to CTRLiPSC-CMs. The observed differences persisted under Iso stimulation, which caused a general $T_{50}$ reduction. CTRL-iPSC-CMs showed a higher spark frequency at higher Iso levels $(1 \mu \mathrm{M})$, but overall there was no difference in total $\mathrm{Ca}^{2+}$ leakage. In 3D EHM, iPSCEHM from cell isolates obtained from TTS patients showed a significantly higher sensitivity compared to CTRL-iPSC-EHM when challenged with Iso, as shown by a lower EC 50 . After $24 \mathrm{~h}$ Iso pre-stimulation, the observed $\mathrm{EC}_{50}$ differences were even higher than before, with CTRL-iPSC-EHM exhibiting stronger $\beta$-AR desensitization than TTS-iPSC-EHM. Additionally, changes in beating frequency after $24 \mathrm{~h}$ Iso stimulation were more prominent in TTS-iPSC-EHM compared to CTRL-iPSC-EHM, which is in line with the reported frequency differences in the MEA measurements. Interestingly, TTS-iPSC-EHM showed a lower force development than CTRL-iPSC-EHM without catecholamine stimulation. The TTS-iPSC-CMs exhibited higher lipid accumulation in comparison to CTRL-iPSC-CMs, suggesting a role of lipotoxicity in the disease. 
The selective $\beta$-blockers CGP and ICI were used to uncover the roles of different $\beta$-ARs in CTRL- and TTS-iPSC-CMs during electrical analysis and $\mathrm{Ca}^{2+}$ cycling. CTRL-iPSCCMs showed stronger Iso effects under CGP-based $\beta_{1}$-AR inhibition in $T_{50}$ times and rise times. When TTS-iPSC-CMs were treated with ICI, Iso stimulation caused significant decreases in relative $T_{50}$ times and rise times. MEA experiments demonstrated that, under $\beta_{2}$-AR blockage, CTRL-iPSC-CMs showed a reduced response towards Iso stimulation. These findings underpin an important role of $\beta_{2}$-ARs in CTRL-iPSC-CMs and $\beta_{1}$-ARs in TTS-iPSC-CMs.

Overall, TTS-iPSC-CMs and TTS-iPSC-EHM reacted stronger towards Iso stimulation and exhibited a pronounced $\beta_{1}-A R$ reaction in comparison to CTRL-iPSC-CMs. Furthermore, TTS-iPSC-CMs showed differences versus CTRL-iPSC-CMs even without Iso stimulation, suggesting a fundamental difference to CTRL-iPSC-CMs, which could be caused by a genetic predisposition in TTS.

\subsection{Stimulation with Iso did not induce significant levels of apoptosis}

Here, it was shown that treatment of TTS-IPSC-CMs with high catecholamine concentrations did not induce significantly different levels of apoptosis compared to untreated CTRL-iPSC-CMs. This suggests that the catecholamine levels used in this study are not able to activate acute apoptosis in cardiomyocytes. This observation is in line with the fact that $\beta$-ARs stimulated by catecholamines can activate $G_{i}$ proteins (Heubach et al., 2004; Paur et al., 2012) and may modulate myocardial damage during a catecholamine crisis. In TTS patients, PI3K expression was elevated and AKT phosphorylation was increased, thereby possibly contributing to a cellular protective mechanism (Nef et al., 2009a). It was found by our group as well as several others that $\beta$ AR stimulation increases NR4A1-expression (Borchert et al., 2017; Medzikovic et al., 2015; Yan et al., 2015). Additionally, our group demonstrated a higher response of NR4A1 towards Iso in TTS-iPSC-CMs compared to CTRL-iPSC-CMs (Borchert et al., 2017). You et al. connected NR4A1 towards apoptosis by demonstrating that NR4A1 upregulates 
latexin-3 and suppresses Iso-induced apoptosis (You et al., 2018). The high expression of NR4A1 in CTRL- and TTS-iPSC-CMs is in line with these findings. Concerning the patient situation, it must be stated that cardiac biomarkers like troponin are elevated during a TTS event suggesting cell damage (Templin et al., 2015). However, the increase of elevated troponin is usually lower in TTS than in STEMI(Ghadri et al., 2018b).

\subsection{Iso stimulation of iPSCs causes lipid accumulation}

The TTS-iPSC-CMs exhibited higher lipid accumulation after catecholamine treatment in comparison to CTRL-iPSC-CMs. These findings are in line with observations from a study by Shao et al., demonstrating lipid accumulation upon Iso exposure in human cardiac biopsies and the HL-1 cardiomyocyte cell line (Shao et al., 2013). Furthermore, the same group demonstrated that patients' serum was able to induce the same effect as Iso (Shao et al., 2013). The results are underpinned by higher expression of the CD36 lipid transporter and reduced expression of CPT1C lipid translocase in TTS-iPSC-CMs by our group. Additionally, Jodalen and coworkers demonstrated an extensive formation of intracellular lipid droplets and serum fat accumulation in mice treated with Iso, concordant to the results in this work (Jodalen et al., 1982). Interestingly, excess intracellular lipids in cardiomyocytes can activate protein kinase $C$ and cause $\beta$-AR downregulation, resulting in reduced cAMP levels (Drosatos et al., 2011). Based on these results, one could argue that $\beta$-response in TTS-iPSC-CMs would decrease with time, when the cells accumulate higher amounts of lipids. This was not observed in the obtained iPSC-EHM results showing a lower $\mathrm{EC}_{50}$ increase towards Iso after $24 \mathrm{~h}$ Iso pre-stimulation in TTS-iPSC$\mathrm{CMs}$ and, therefore, lower $\beta$-AR desensitization. The change in beating frequency after $24 \mathrm{~h}$ iso pre-stimulation was bigger in TTS-iPSC-CMs and is not in line with the lower cAMP level results of Drosatos and colleagues. Future experiments would focus on the combination of TTS patients' serum and TTS-iPSC-CMs to replicate the results of Shao et al. using our model and identify potential contributing factors. Additionally, Förster resonance energy transfer (FRET) analysis of Iso pre-stimulated iPSC-CMs would shed light on the consequences of lipid accumulation on cAMP levels. 


\subsection{Electrical activity analysis shows that TTS-iPSC-CMs react stronger towards Iso than CTRL-iPSC-CMs}

The TTS-iPSC-CMs and CTRL-iPSC-CMs showed significant electrophysiological differences on many levels.

When stimulated with rising levels of catecholamines, TTS-iPSC-CMs and CTRL-iPSCCMs stopped spontaneous electrical activity. However, the catecholamine levels needed for TTS-iPSC-CMs to achieve this effect were lower than in CTRL-iPSC-CMs. This behavior is in line with the theory of an increased susceptibility towards the negative effects of catecholamines in cells from individuals with TTS. This behavior can be interpreted as cardiac stunning and therefore mimics the behavior of ventricular dysfunction observed in TTS-patients (Templin et al., 2015). In parallel to TTS patients, electrical silent iPSC-CMs showed beating recovery $1 \mathrm{~h}$ to $24 \mathrm{~h}$ after the removal of catecholamines. The observed recovery was significantly faster than in vivo and occurs in the timescale of hours compared to days in actual patients. In line with our findings, a significantly faster recovery was also seen in in vivo rat experiments with catecholamines being administered from an external source (Paur et al., 2012). In a retrospective study, ejection fraction recovery time in TTS was found to be 25 days (Shaikh et al., 2018). Interestingly, in the same study, a high variability in recovery times was noted (Shaikh et al., 2018). A long recovery time was also seen by Lee and colleagues, who found improvements in the ejection fraction up to 5 weeks after hospital discharge (Lee, 2020). The rapid recovery in the iPSC-CMs might be in part due to the fast and thorough removal of catecholamines in the artificial system, whereas, in patients, catecholamine levels stay high for longer durations. This has been demonstrated by Christensen et al., who found that TTS patients still had increased plasma Epi levels at 100 days after their admission compared to controls (Christensen et al., 2016). One could also speculate that catecholamine levels in TTS patients are on a higher basal level.

As expected, rising catecholamine concentrations caused an increased beating frequency. When challenged with increasing dosages of catecholamines, a higher 
increase in the beating frequency was observed in TTS-iPSC-CMs in comparison with CTRL-iPSC-CMs. It was shown that TTS patients had significantly increased heart rates in comparison to acute MI patients, which is in line with our results (Templin et al., 2015). The importance of a high heart rate in TTS was also underpinned by a study of Ali and colleagues who showed that pharmacological suppression of the sinoatrial node was able to ameliorate a TTS-like phenotype, which was induced by bolus Iso injection in rats (Ali et al., 2020). Concerning the comparability of our results, Wells and colleagues used human iPSC-CMs in a comparative study using MEAs (Wells et al., 2019). Stimulation with $1 \mu \mathrm{M}$ Iso resulted in a frequency increase of $50 \%$ for 44 -day-old iPSC-CMs, which is in the same range as the CTRL-iPSC-CMs in this work (100\% increase). Surprisingly, 69 day-old iPSC-CMs were nearly unresponsive towards Iso, at least in part, contradicting the results observed in this work (Wells et al., 2019). Additionally, their observed basal frequencies were higher, being in the range of $2 \mathrm{~Hz}$ in 2-month-old iPSC-CMs compared to $0.5 \mathrm{~Hz}$ in this work. In another publication, which used less than one-month-old hESCCMs frequencies of $0.3 \mathrm{~Hz}$ to $1 \mathrm{~Hz}$ were found (Zhu et al., 2017). Furthermore, heart rates in non-stressed humans are typically in the range of $1 \mathrm{~Hz}$, being in the same range as the iPSC-CMs used in this work. Moreover, at very high catecholamine levels, iPSC-CMs showed a reduction in beating frequencies. This is in line with the publication of Paur et al., who showed that by activation of inhibitory $G_{i}$ proteins, catecholamines can indeed lower the heart rate (Paur et al., 2012). Additionally, Heubach and colleagues showed that Epi can activate $G_{s}$ and $G_{i}$ proteins via $\beta_{2}$-ARs, supporting the results observed (Heubach et al., 2004). Izumi and colleagues infused Epi in monkeys and observed apical dyskinesia (corresponding to electrical silencing) and an overall frequency increase (Izumi et al., 2009).

The observed field potential durations did increase under Iso stimulation and no difference between CTRL-iPSC-CMs and TTS-iPSC-CMs was found. The observed values are higher than in other reports using 25-day old hESC-iPSC-CMs (Zhu et al., 2017) with 300 ms and 70-day-old human iPSC-CMs with $200 \mathrm{~ms}$ (Wells et al., 2019). In this work, neither relevant FPD prolongations nor significant differences in FPD between CTRL- and TTSiPSC-CMs were observed. TTS is associated with a QT-interval prolongation and dangerous arrhythmic events are seen at QTc-intervals of $500 \mathrm{~ms}$ or more (Brown et al., 
2015; Ghadri et al., 2018b). However, not all TTS patients experience QT prolongation (Ghadri et al., 2018b; Templin et al., 2015).

The absolute conduction speeds were $11.42 \mathrm{~cm} / \mathrm{s}$ for CTRL-iPSC-CMs and $4.97 \mathrm{~cm} / \mathrm{s}$ for TTS-iPSC-CMs, which differed significantly. The results are in the observed range of iPSC-CMs in culture, as shown by Zhu and colleagues, who reported a conduction velocity of $9 \mathrm{~cm} / \mathrm{s}$ for 25-day-old and $12 \mathrm{~cm} / \mathrm{s}$ for 28-day-old hESC-CMs (Zhu et al., 2017). Of note, the basal conduction velocity was reduced in TTS-iPSC-CMs compared to CTRL-iPSCCMs. This finding needs further confirmation due to the low $n$ numbers and possible maturation differences between the two groups of iPSCs, which may have affected the conduction velocity. If this assumption is confirmed, conduction velocity differences might play a role in TTS in the form of conduction disturbances, blocks, or reentry events, which ultimately cause malignant ventricular arrhythmias, frequently observed in TTS patients (Bonello et al., 2008; Brown et al., 2015; Ghadri et al., 2018b).

\subsection{Differences in $\mathrm{Ca}^{2+}$ handling}

Overall, the results show $\mathrm{Ca}^{2+}$ handling differences between TTS-iPSC-CMs and CTRLiPSC-CMs on multiple levels. Despite increased rise times, $\mathrm{Ca}^{2+}$ handling was significantly faster under basal conditions with a significantly reduced $T_{50}$ in TTS-iPSC-CMs compared to CTRL-iPSC-CMs.

Upon Iso stimulation, $T_{50}$ decreased by about $20 \%$ compared to basal levels. Comparing the results with other studies, the reduction in $T_{50}$ times upon Iso stimulation is similar to the results found by Wu et al. in 60-day-old human iPSC-CMs, which was $20 \%$ after $1 \mu \mathrm{M}$ Iso stimulation (Wu et al., 2015). It is also noteworthy that they showed that the $T_{50}$ and rise times were differentiation dependent, with effects being stronger at shorter differentiation times (Wu et al., 2015). During acute TTS events, the SERCA protein level was reduced, causing an increased PLN/SERCA ratio (Nef et al., 2009b). This would result in slower $\mathrm{T}_{50} \mathrm{Ca}^{2+}$ kinetics and contradict the observed low $\mathrm{T}_{50}$ times in TTS-iPSCCMs. The iPSC-CMs measured in this work did not have time to change protein expression in response to Iso. Phosphorylation events are faster and indeed, our group 
observed stronger PLN-S16 phosphorylation under Iso stimulation in TTS-iPSC-CMs compared to CTRL-iPSC-CMs (Borchert et al., 2017). This would result in lower SERCA inhibition and consequently lower $T_{50}$ times, which were observed.

We found significantly higher rise times in TTS-iPSC-CMs than in CTRL-iPSC-CMs under basal conditions. There was no significant increase or decrease caused by Iso stimulation. This is in contrast to the aforementioned study of Wu and colleagues, which showed a reduction of time to peak times after Iso stimulation of iPSC-CMs by $20 \%$ (Wu et al., 2015). However, it must not go unmentioned that they used non-pacing experiments, whereas the data obtained here were from paced cardiomyocytes.

In TTS-iPSC-CMs, an overall higher transient $\mathrm{Ca}^{2+}$ level was present at basal levels compared to CTRL-iPSC-CMs. In contrast to CTRL-iPSC-CMs, TTS-iPSC-CMs were not able to exhibit an increase in $\mathrm{Ca}^{2+}$ levels as a response to Iso. Furthermore, TTS-iPSCCMs transients shrank in response to high Iso dosages. This negative inotropic response (akinetic parts) is in line with the TTS phenotype (Templin et al., 2015). The observed behavior of transient shrinking in TTS-iPSC-CMs is in line with other studies showing that high Iso levels cause a decline in beating capacity and supports the theory of a dominant role of $\mathrm{G}_{\mathrm{i}}$ proteins at high Iso levels in TTS (Paur et al., 2012). Surprisingly, the increased $\mathrm{Ca}^{2+}$ transients in TTS-iPSC-CMs without Iso stimulation did not result in stronger contraction force but resulted in the opposite: a reduction in force when challenged with higher $\mathrm{Ca}^{2+}$ concentrations in the medium in TTS-iPSC-EHM. It is worth mentioning that the CTRL-iPSC-CMs also exhibited a decrease of $\mathrm{Ca}^{2+}$ levels under high Iso stimulation compared to medium Iso levels $(1 \mu \mathrm{M})$. This decrease, however, was smaller in comparison to TTS-iPSC-CMs. This underpins the theory of a higher vulnerability in TTS patients.

The total $\mathrm{Ca}^{2+}$ leakage was not different between CTRL- and TTS-iPSC-CMs.

\subsection{EHM from TTS-iPSC-CMs show a higher sensitivity for Iso}


When challenged with Iso, EHM constructed from TTS-iPSC-CMs showed a significantly higher sensitivity compared to CTRL-iPSC-EHM, as shown by a lower EC 50 . After $24 \mathrm{~h}$ Iso pre-stimulation, the observed $\mathrm{EC}_{50}$ differences were even higher than before, with CTRL-iPSC-EHM exhibiting a stronger EC $_{50}$ increase than TTS-iPSC-EHM. Therefore, $\beta$ AR desensitization upon Iso stimulation was present in both IPSC-EHM but stronger in CTRL-iPSC-EHMs. Additionally, beating frequency changes after $24 \mathrm{~h}$ Iso stimulation were higher in TTS-iPSC-EHM compared to CTRL-iPSC-EHM, which is in line with the reported frequency differences in the MEA measurements. Therefore, EHM measurements are in line with the previous findings suggesting a generally stronger reaction of TTS-iPSC-CMs towards Iso. The lower desensitization in TTS-iPSC-EHM is especially interesting. It is in line with the fact that many SNPs in $\beta$-ARs (Vriz et al., 2011; Zaroff et al., 2006) themselves or $\beta$-regulating proteins like GRK5 (Spinelli et al., 2010) were found. To put this in a whole picture: The high catecholamine levels seen in TTS patients (Wittstein et al., 2005), in combination with a disturbed shutdown of $\beta$-adrenergic signaling, could very well work together in a two-hit model to cause the disease. Nakano et al. recently published a study showing that GRK2 and $\beta$-arrestin were present at a high level in the cytoplasm and at the membrane of cardiomyocytes from TTS-patients (Nakano et al., 2018). They postulate that GRK2 and $\beta$-arrestin activity contributes to a $G_{s}$-to-Gi switch, which may contribute to the pathophysiology of TTS (Nakano et al., 2018).

Interestingly, TTS-iPSC-EHMs showed a lower force development than CTRL-iPSC-EHM without catecholamine stimulation in response to rising medium $\mathrm{Ca}^{2+}$ levels. This is not in line with the increased $\mathrm{F} / \mathrm{FO} \mathrm{Ca}^{2+}$ transients observed at basal levels in TTS-iPSC-CMs in comparison to CTRL-iPSC-CMs.

\subsection{CGP and ICI shed light on the role of different $\beta$-ARs}

Experiments with the selective $\beta_{1}$-inhibitors CGP and selective $\beta_{2}$-inhibitors ICI revealed that $\beta_{2}$-ARs played a more important role in CTRL-iPSC-CMs, whereas $\beta_{1}$-ARs were particularly engaged in TTS-iPSC-CMs, as judged from the MEA and $\mathrm{Ca}^{2+}$ experiments. The AR preferences are in line with results from our lab as found in a FRET setup 
measuring cAMP levels (Borchert et al., 2017). These measured effects are all associated with $G_{s}$ activity. Since Paur et al. stressed the importance of $G_{i}$ proteins in TTS (Paur et al., 2012), it would be of interest to check for $\mathrm{G}_{\mathrm{s}}$-to- $\mathrm{G}_{i}$ switch differences in CTRL- and TTS-iPSC-CMs in the future. It must be noted that the presence of CGP, ICl, or both was enough to cause significant changes in $\mathrm{Ca}^{2+}$ kinetics. Therefore, the analysis was done normalized $\mathrm{T}_{50}$ and rise times to exclude the effects of CGP or ICl. The different reactions towards CGP and ICl could, in principle, be caused by different $\beta_{1}$ - and $\beta_{2}$-AR expression. Wu et al. found increasing $\beta-A R$ expression in developing iPSC-CMs (Wu et al., 2015). They also found that $\beta_{2}$-AR-expression was already upregulated in iPSC-CMs after 12 days of culture, whereas $\beta_{1}$-AR-expression kept increasing until iPSC-CMs reached day 60. Therefore, the use of iPSC-CMs older than 2 months seems to be the right choice to achieve the proper expression of the two $\beta$-AR proteins. The combination of CGP and ICI greatly suppressed the response of iPSC-CMs towards Iso. This suggests that $\beta_{1}$ - and $\beta_{2-}$ ARs are the main drivers of Iso reactions involved in the heart. The role of $\beta_{3}-A R s$, which have been shown to have a negative effect on the heart contractility by nitric oxide and cGMP production (Gauthier et al., 1998) and might be cardioprotective (Cannavo and Koch, 2017) remains elusive and seems to be relatively small based on the CGP and ICI data. However, specific $\beta_{3}-A R$-stimulation of CTRL- and TTS-iPSC-CMs could yield interesting results in the future.

This complexity is supported by a different expression of $\beta$-ARs in the heart with a higher $\beta_{1}$ - to $\beta_{2}-A R$ ratio in ventricles than in atria (Steinfath et al., 1992). Both, the different $\beta$ $\mathrm{AR}$ distribution and the complex 3D-form of the heart could be overcome by the use of cardiac organoids. Different $\beta$-responses can be caused by different CM microdomains, as demonstrated by Wright and colleagues, who found that basal CMs had more shortlived cAMP responses to $\beta_{2}$ stimulation (Wright et al., 2018).

\subsection{The genetic component of TTS}


The different behavior of TTS-iPSC-CMs and CTRL-iPSC-CMs supports the theory of a genetic background of the disease. Whole-exome sequencing experiments, which were performed in cooperation with Prof. B. Wollnik (Human Genetics, UMG), found mutations in CASQ2 in the TTS5 patient (Borchert et al., 2017). This is the first study where CASQ2 variants were suggested to be the genetic cause for the development of TTS. In support of a genetic origin, former studies described several mutations associated with TTS. Vriz et al. showed an association with variants in ADRB1 and ADRB2 (Vriz et al., 2011). Furthermore, variants of BAG3 resulting in reduced binding of miRNA-371a-5p (d'Avenia et al., 2015) and GRK5, which is known for $\beta$-AR desensitization (Liggett et al., 2008; Spinelli et al., 2010), have been linked to the development of TTS. In contrast to these findings, a recent case-control study showed a lack of association between TTS cases and mutations in ADRB1, BAG3, and GRK5 (Mattsson et al., 2018). Despite these incoherent observations, the different behaviors between TTS-iPSC-CMs and CTRLiPSC-CMs point towards a certain genetic predisposition. Indeed, Kumar et al. report a TTS family with a woman and her mother both suffering from TTS, while two reports described two sisters with TTS, further supporting the hypothesis that genetic factors may contribute to the etiology of the disease (Pison et al., 2004; Kumar et al., 2010; Ikutomi et al., 2014). However, until today TTS is not completely proven to be an inheritable condition. In light of the gathered data, one could theorize that, in principle, all humans are prone to getting TTS and the only distinction between TTS patients and non-patients might be some factors that lower the catecholamine threshold level for developing a TTS event. This is supported by the fact that all models used wildtype animals (Ali et al., 2020; Izumi et al., 2009; Paur et al., 2012) and a high catecholamine level is all that is needed to trigger a TTS phenotype. This can, of course, also be interpreted as a lack of genetic predisposition. The obtained results stress that CTRL- and TTS-iPSC-CMs behave differently and thereby suggest a genetic component in the disease. If a genetic predisposition exists, the aforementioned studies suggest that the nature of a predisposition is most likely multifactorial. 


\subsection{TTS triggering catecholamine levels differ between models and patients}

Here in this study, we used catecholamine concentrations between $100 \mathrm{nM}$ and $5 \mathrm{mM}$ for $2 \mathrm{~h}$ in most experiments. It is obvious that concentrations of $5 \mathrm{mM}$ are supra-physiological. However, supraphysiological high amounts of catecholamines were also used in studies in rats and monkeys to trigger TTS-like events (Ali et al., 2020; Izumi et al., 2009; Paur et al., 2012). Izumi and colleagues, for example, used $10 \mu \mathrm{g} /\left(\mathrm{min}^{*} \mathrm{~kg}\right)$ Epi infusion to trigger a TTS phenotype (Izumi et al., 2009), while Epi plasma levels during subacute TTS were reported to be $70 \mathrm{pg} / \mathrm{ml}$ (Christensen et al., 2016). The named studies show a rather strong response in the form of apical ballooning compared with the herein presented findings. The reasons for this could be the different species or the difference in the used models (in vivo vs. in vitro). Interestingly, TTS could be triggered in different animals, arguing for the theory that with high enough levels of catecholamines, TTS can indeed be triggered in any individual. Christensen et al. reported significantly increased plasma Epi levels in subacute TTS patients in the range from $70 \mathrm{pg} / \mathrm{ml}$ during the subacute phase and $38 \mathrm{pg} / \mathrm{mL}$ during recovery in comparison to myocardial or endocarditis patients with similar symptoms having Epi levels of $44 \mathrm{pg} / \mathrm{ml}$ during subacute phase vs. $24 \mathrm{pg} / \mathrm{ml}$ during recovery (Christensen et al., 2016). Furthermore, the actual catecholamine levels during the onset of the TTS attack are largely unknown and may be higher than the levels measured in the hospital. It must be taken into careful consideration that blood catecholamine levels do not necessarily represent the catecholamine levels at the cardiomyocyte itself since direct sympathetic innervation is also present. Indeed, Kume et al. reported increased catecholamine release from sympathetic neurons in TTS cases (Kume et al., 2008).

\subsection{Limitations}

Although this work uncovers some new insights into the pathology of TTS, there are limitations to be considered. 
Firstly, an iPSC-based system is, by its definition as it is not a naturally occurring cell type in the human body. Consequently, cells differentiated from this cell type may be different in several properties. ESCs, however, occur naturally with close similarities to iPSCs (Takahashi et al., 2007). Furthermore, the differentiation process from iPSCs to cardiomyocytes is, while related to the natural differentiation process, an artificial process, which is much shorter. It yields cardiomyocytes, which show significant differences to adult human cardiomyocytes. In fact, these cardiomyocytes are closer to embryonic cardiomyocytes than mature adult ones (Lundy et al., 2013). This may cause different results as TTS is a disease of the elderly. To obtain more mature cardiomyocytes, the approach of longer cultivation times (Lundy et al., 2013) and cultivation on a geltrex matrix (Parikh et al., 2017) was used in this work. Here, multinucleation, as a marker of maturation, was regularly observed in the iPSC-CMs. Synchronized electrical activity is another sign of maturation (Scuderi and Butcher, 2017), which was observed in the iPSCCMs used in this work. The iPSC-CMs showed conduction velocity, frequency increase towards catecholamines, and $\mathrm{Ca}^{2+}$ handling similar to iPSC-CMs used in other studies (Wells et al., 2019; Wu et al., 2015; Zhu et al., 2017). Further maturation indicators, such as the presence of a T-tubule network, would be a good addon for future maturation assessments.

The main limitation of this investigation is that only a restricted number of cell isolates were tested. Thus, a much higher number of samples from both TTS patients and casematched controls are required to confirm these preliminary results.

Another fact that needs mentioning is that a human heart does not consist of pure myocytes but rather a complex interplay between cardiomyocytes, fibroblasts, pacemaker cells, endothelial cells, and other cells organized in a complex 3D structure. Another point is obviously that a complex blood vessel system cannot be simulated in vitro yet. In consequence, the vasospasm component of TTS cannot be evaluated under cell culture conditions without proper vascularized 3D cell models. A future approach is the co-culture of iPSC-CMs and iPSC-derived neurons in 2D or heart brain-organoids.

The influence of the heart-brain axis, which is important in TTS, cannot be modeled in a pure iPSC system. 
Another issue, which needs consideration is the percentage of different cardiac cell types yielded by the used differentiation protocol. While more than $80 \%$ of cells were cTnT positive, their exact type of iPSC-CM was not confirmed by patch-clamp. It is likely that some cells were of the atrial or nodal type, which could influence the results. By adapting protocols to obtain purer CM cell types, usage of pure ventricular CMs is possible in the future (Kolanowski et al., 2017; Weng et al., 2014). Furthermore, the reaction of atrialCMs could be studied as atria are sometimes affected in TTS and associated with a worse outcome (Eitel et al., 2011; Ghadri et al., 2018a). In addition, the use of multi-electrode arrays requires multiple preparation steps from digestion over the seeding of cells towards measuring, which may add to the complexity of this approach. Hopefully, with the advance of new materials and methods, this may be reduced in future experiments. Unfortunately, the available control probands were not as closely matched towards the TTS patients concerning the age as would have been desirable. Therefore, a future TTS-attack in the control probands cannot be excluded. However, it must be stated that optimal controls such as elderly women after menopause who went through very stressful events are not easy to obtain. This is a common problem when working with iPSCs. In future studies, better controls may be available due to a massive effort to build iPSC biobanks with a variety of probands.

An additional challenge is the high levels of catecholamines, which are necessary to model the disease. In summary, it must be stated that there are still tremendous obstacles to be overcome to achieve a highly reproducible and reliable TTS model.

\subsection{Clinical implications}

As mentioned in the introduction, the role of $\beta$-blockers in TTS is controversial (Ghadri et al., 2018b; Isogai et al., 2016; Sharkey et al., 2010). In this work, most of the Iso effects were inhibited by a combination of CGP and $\mathrm{ICl}$. This in line with studies showing $\beta$ blockers to be effective in animal models (Izumi et al., 2009; Ueyama et al., 2002). It is

worth to note that in our experimental setting with $\beta$-blockers, Iso was added after a preincubation with the respective blocker, thereby abolishing Iso actions from the start. In 
patients, this is usually not the case as $\beta$-blockers are given after hospitalization. Following this logic, $\beta$-blockers should prevent further TTS attacks, however, this does not seem to be the case (Santoro et al., 2013). Another mystery is a possible association of TTS with cancer (Girardey et al., 2016; Sattler et al., 2017; Sattler et al., 2018). It is speculated that excessive catecholamine levels are associated with both diseases (Sattler et al., 2018). From this point of view, long-term Iso exposure of TTS-iPSCs could be of interest. This could also be connected to increased ROS levels followed by exposure to high catecholamines in TTS (El-Battrawy et al., 2018; Zhang et al., 2017) as ROS can also damage DNA and thus contribute towards cancer development. The used iPSC-CMs are, in principle, suited for ROS measurements, as demonstrated by Haupt et al. (Haupt et al., 2020). Consequently, ROS measurements should be part of the model. As mentioned in the introduction, the presence of microvascular spasms is a very suitable theory to explain TTS (Abdelmoneim et al., 2009; Galiuto et al., 2010). This can, of course, not be modeled in the herein used model, as it would require complex and very well-defined organoids or better in vivo studies.

\subsection{Conclusion}

From the targeted aims, the following could be achieved:

A high number of CTRL- and TTS-iPSC were successfully reprogrammed and integrationfree reprogramming methods were established and optimized. The culture was switched towards feeder-free conditions to enhance reproducibility. Feeder-free differentiation to iPSC-CMs was successful and yielded high purity iPSC-CMs, which fulfilled some maturity criteria. In 2D culture, iPSC-CMs were treated with catecholamines and apoptotic analysis and $\mathrm{Ca}^{2+}$ imaging was established. MEA measurements were improved, 2D conduction velocity analysis was established and performed. Lipid measurements were optimized and carried out successfully. With the help of cooperation partners, 3D models in the form of iPSC-iPSC-EHM were produced and the impact of catecholamines on these models was analyzed. Using selective $\beta$-inhibitors, the role of $\beta_{1-}$ and $\beta_{2}$-ARs in CTRL- 
and TTS-iPSC-CMs were explored. The overall produced model recapitulated some, but not all TTS phenotypes and can be the basis for future developments.

\subsection{Outlook}

Future studies may further increase our knowledge of TTS. These could be done with the help of organoids creating a more natural environment. Furthermore, it may be worthwhile to study genetic predispositions underlying the disease. After model optimization, a high throughput drug screening would be possible to identify effective countermeasures against the effect of high concentrations of catecholamines.

In addition, genetically engineered iPSC-CMs should be developed, which express only $\beta_{1-}, \beta_{2-}$ or both AR types. The aforementioned different behaviors under specific pharmacological $\beta_{1}$ - or $\beta_{2}$-stimulation support this approach. Another possible way is the use of explanted human hearts as cardiomyocyte source. This, however, has the drawback that cardiomyocytes from failing hearts are already pathologically degenerated and may therefore be of limited use. Another approach would be the exploration of TTSassociated biomarkers, which has already started by the identification of certain miRNAs (Jaguszewski et al., 2014) to specifically differentiate TTS from a STEMI. While the model generated in this thesis focuses on rather short timescales, some TTS patients seem to show a longer timeframe to develop symptoms (Ghose and Banerjee, 2019; Stöllberger and Finsterer, 2019). These observations suggest a cumulative toxic effect of catecholamine. It is therefore of interest to know how the catecholamine levels of TTS patients develop over time and whether this has an influence on the length of the TTSattack. It is also interesting to know whether TTS patients have a generally higher level of catecholamines before disease onset. Considering the long-term elevated serum catecholamine levels (Christensen et al., 2016) in TTS patients after an event, this may indeed be the case. 


\section{Appendix}

A

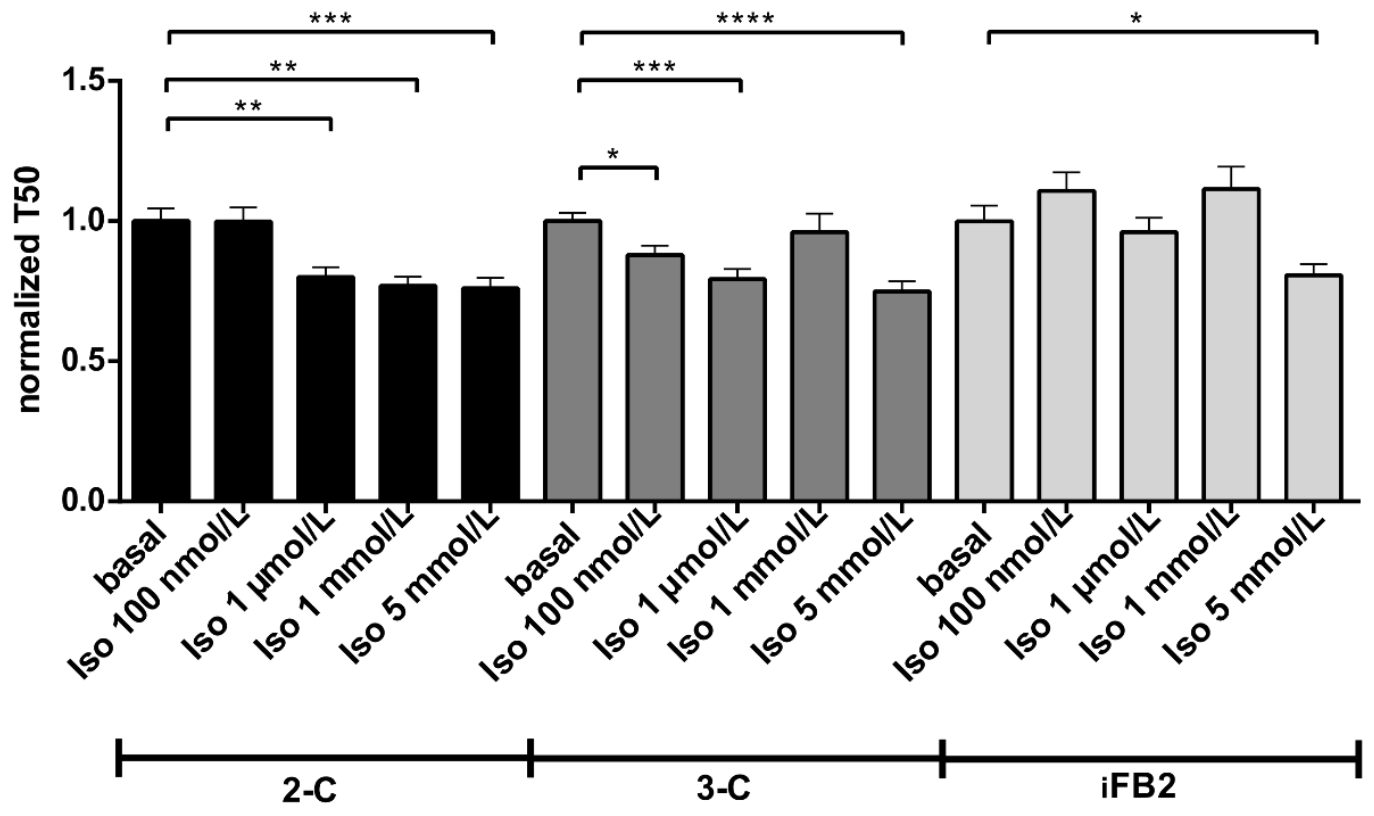

B

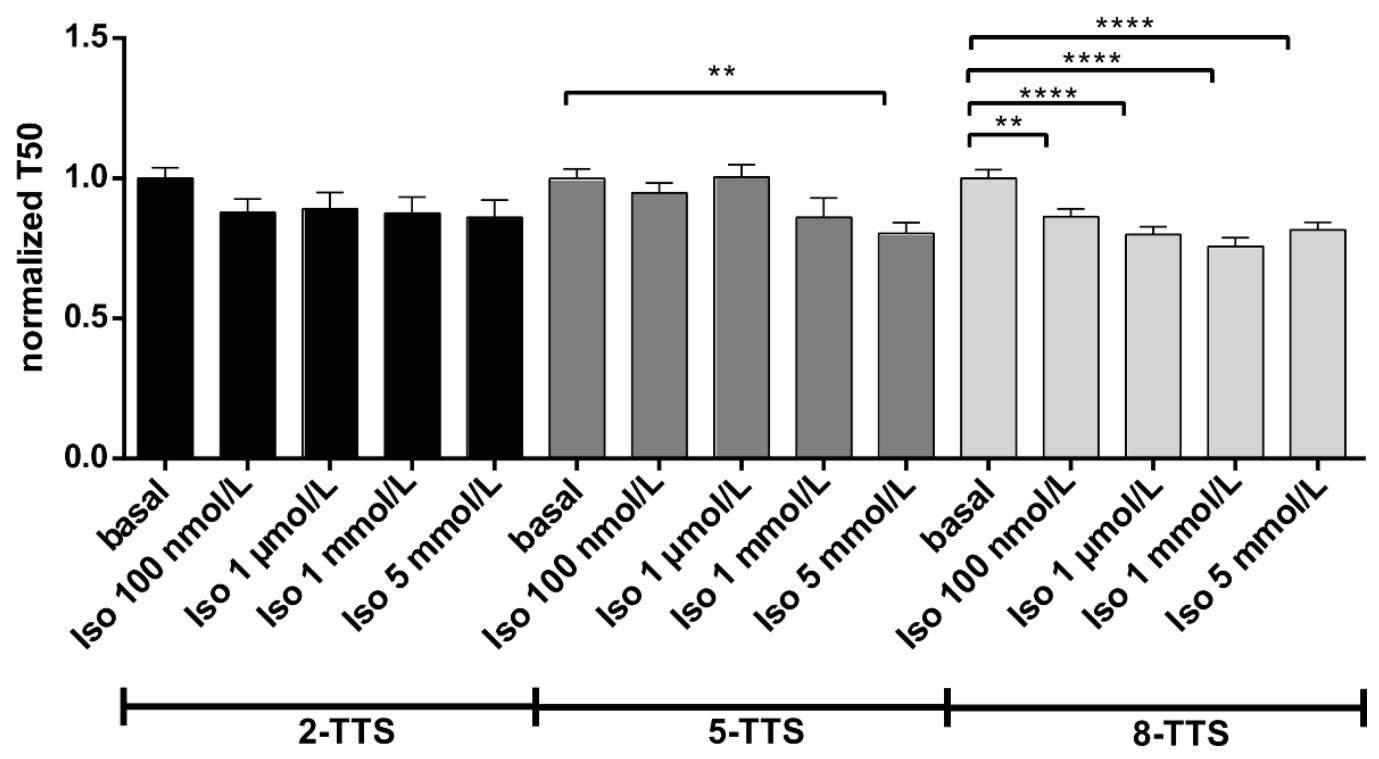

Supplemental Figure 1: A: Normalized $T_{50}$ measurements under Iso stimulation of all used CTRL-iPSC-CMs (2-C [ $n=47$ cells], 3-C [ $n=67$ cells], iFB2 [ $n=39$ cells]). B: Normalized $T_{50}$ measurements under Iso stimulation of all used TTS-iPSC-CMs (2-TTS [ $n=26$ cells], 5-TTS $[\mathrm{n}=60$ cells], 8-TTS [ $\mathrm{n}=62$ cells]). Significances were calculated using one-way ANOVA. 
A

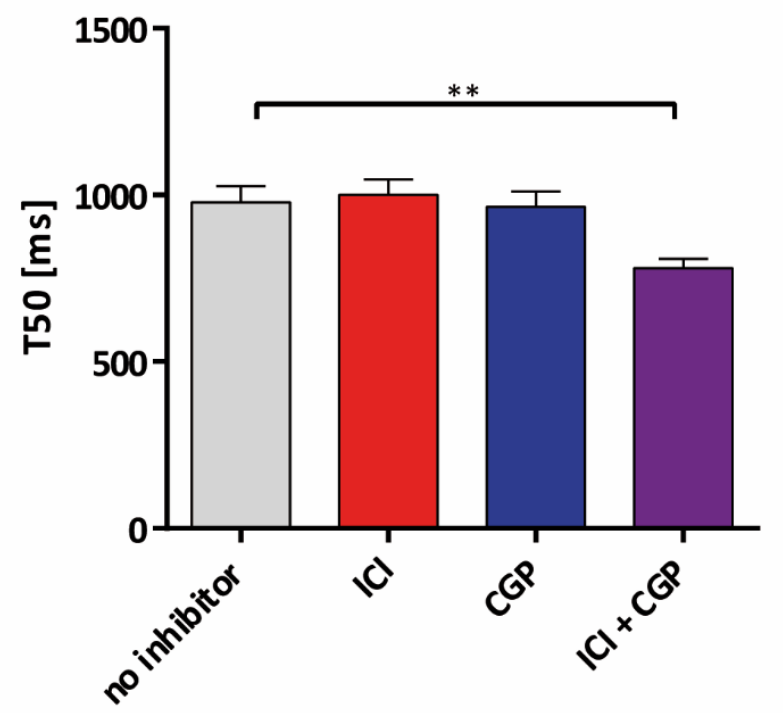

B

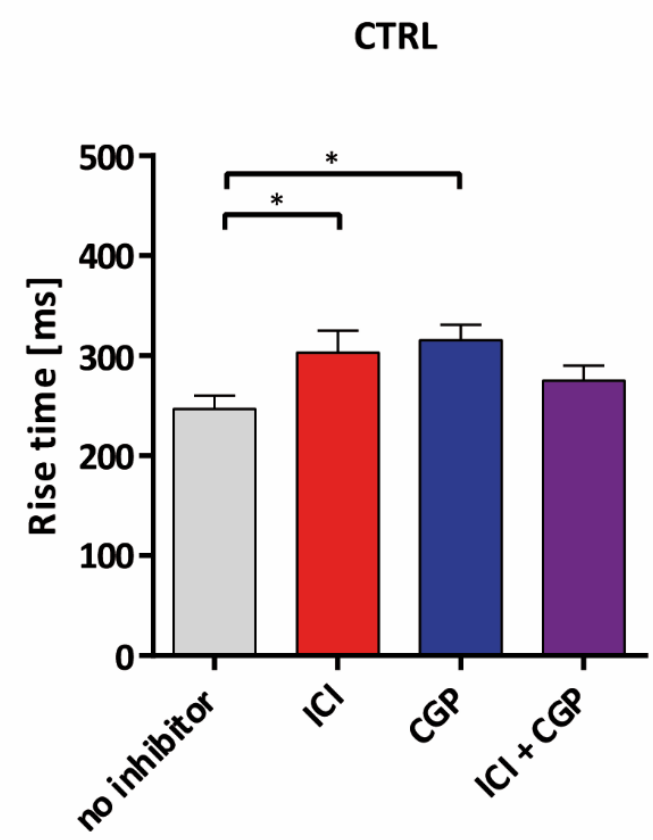

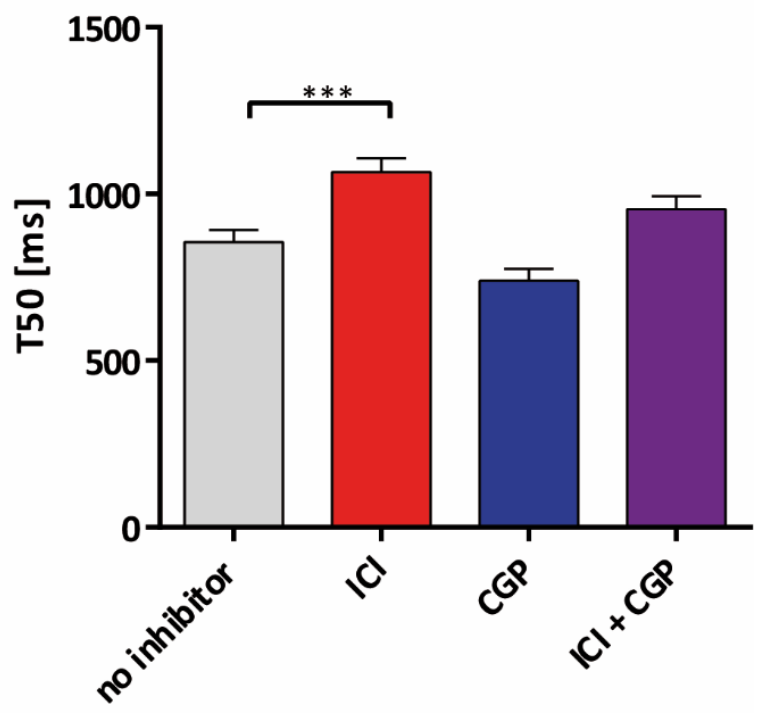

TTS

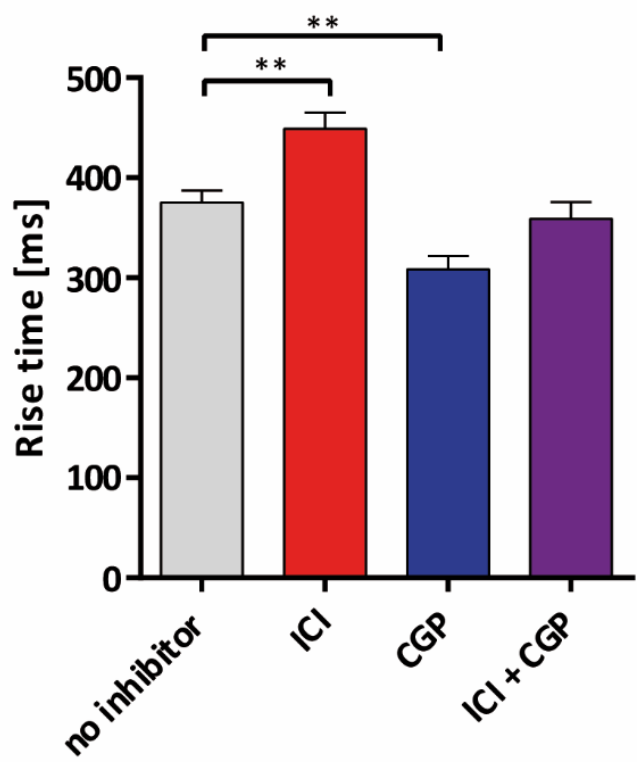

Supplemental Figure 2: A: Absolute $T_{50}$ times without Iso stimulation of $C T R L-(n=69$ cells: $2-C$ [ $n=25$ cells], 3-control [ $n=22$ cells], iFB2 [ $n=22$ cells]) and TTS-iPSC-CMs ( $n=73$ cells: 2 -TTS

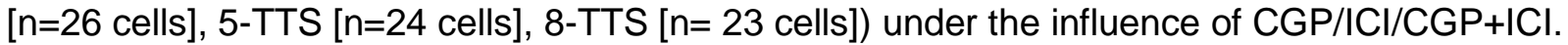
B: Absolute $T_{50}$ times without Iso stimulation of CTRL- ( $n=69$ cells: $2-C$ [ $n=25$ cells], 3-C [ $n=22$ cells], iFB2 [ $n=22$ cells]) and TTS-iPSC-CMs ( $n=73$ cells: 2 -TTS [ $n=26$ cells], 5-TTS [ $n=24$ cells], 8-TTS $[n=23$ cells]) under the influence of CGP/ICI/CGP+ICI. Significances were calculate using one-way ANOVA. 
A

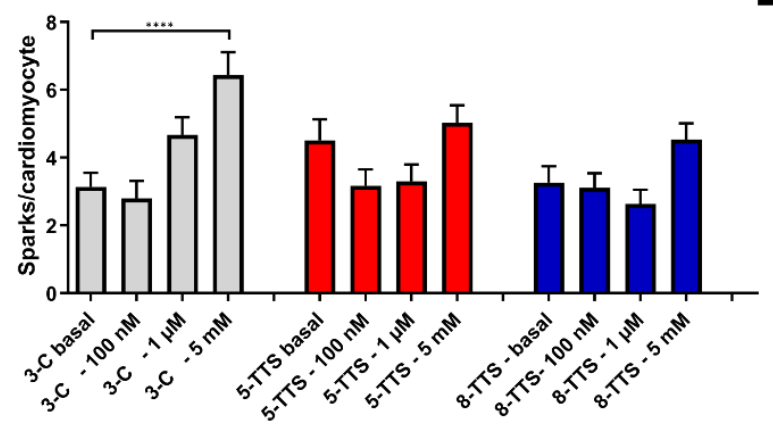

C

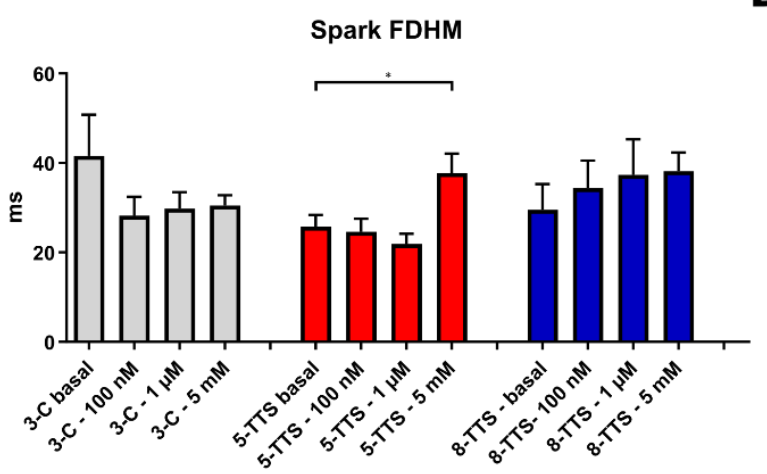

B

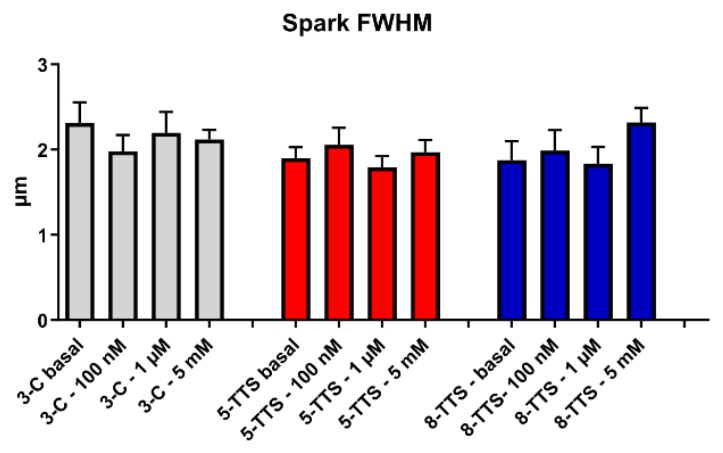

D

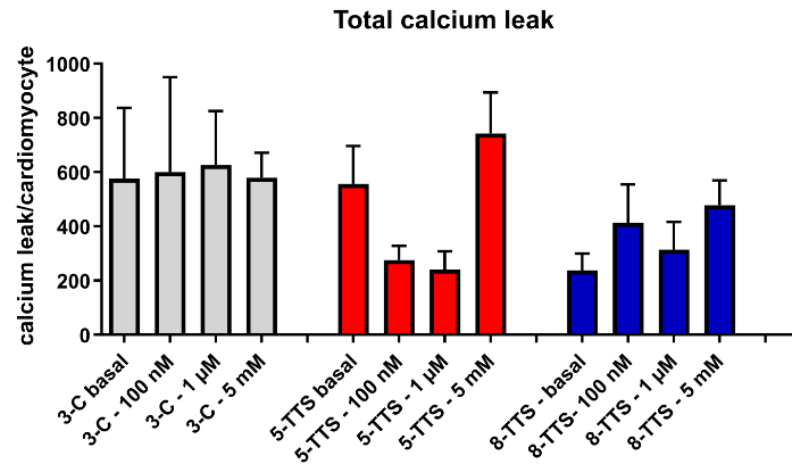

Supplemental Figure 3: A: Spark frequency per cardiomyocyte for the single probands 2-C ( $n=30$ cells from 2 differentiations), 5-TTS ( $n=30$ from 2 differentiations), and 8-TTS ( $n=27$ from 2 differentiations) B: FWHM per cardiomyocyte in the cell isolates from sample 2-C ( $n=30$ cells from 2 differentiations), 5-TTS ( $n=30$ from 2 differentiations) and 8-TTS $(n=27$ from 2 differentiations) C: FDHM frequency per cardiomyocyte from sample 2-C $(n=30$ cells from 2 differentiations), 5-TTS ( $n=30$ from 2 differentiations) and 8-TTS ( $n=27$ from 2 differentiations) D: Total calcium leak per cardiomyocyte as measured in 2-C ( $n=30$ cells from 2 differentiations), 5 -TTS ( $n=30$ from 2 differentiations) and 8-TTS ( $n=27$ from 2 differentiations). Differences were calculated using one-way ANOVA. 


\section{Literature}

Aasen, T., and Izpisúa Belmonte, J.C. (2010). Isolation and cultivation of human keratinocytes from skin or plucked hair for the generation of induced pluripotent stem cells. Nat Protoc 5, 371382.

Abdelmoneim, S.S., Mankad, S.V., Bernier, M., Dhoble, A., Hagen, M.E., Ness, S.A.C., Chandrasekaran, K., Pellikka, P.A., Oh, J.K., and Mulvagh, S.L. (2009). Microvascular function in Takotsubo cardiomyopathy with contrast echocardiography: prospective evaluation and review of literature. J Am Soc Echocardiogr 22, 1249-1255.

Adler, A.F., Cardoso, T., Nolbrant, S., Mattsson, B., Hoban, D.B., Jarl, U., Wahlestedt, J.N., Grealish, S., Björklund, A., and Parmar, M. (2019). hESC-Derived dopaminergic transplants integrate into basal ganglia circuitry in a preclinical model of Parkinson's disease. Cell Rep 28, 3462-3473.e5.

Ahmed, R.E., Anzai, T., Chanthra, N., and Uosaki, H. (2020). A Brief Review of current maturation methods for human induced pluripotent stem cells-derived cardiomyocytes. Front Cell Dev Biol 8.

del Álamo, J.C., Lemons, D., Serrano, R., Savchenko, A., Cerignoli, F., Bodmer, R., and Mercola, M. (2016). High throughput physiological screening of iPSC-derived cardiomyocytes for drug development. Biochim Biophys Acta 1863, 1717-1727.

Ali, A., Redfors, B., Lundgren, J., Alkhoury, J., Oras, J., Gan, L.-M., and Omerovic, E. (2020). The importance of heart rate in isoprenaline-induced takotsubo-like cardiac dysfunction in rats. ESC Heart Fail 7, 2690-2699.

d'Avenia, M., Citro, R., De Marco, M., Veronese, A., Rosati, A., Visone, R., Leptidis, S., Philippen, L., Vitale, G., Cavallo, A., et al. (2015). A novel miR-371a-5p-mediated pathway, leading to BAG3 upregulation in cardiomyocytes in response to epinephrine, is lost in Takotsubo cardiomyopathy. Cell Death Dis 6, e1948.

Avior, Y., Sagi, I., and Benvenisty, N. (2016). Pluripotent stem cells in disease modelling and drug discovery. Nat Rev Mol Cell Biol 17, 170-182.

Badin, R.A., Bugi, A., Williams, S., Vadori, M., Michael, M., Jan, C., Nassi, A., Lecourtois, S., Blancher, A., Cozzi, E., et al. (2019). MHC matching fails to prevent long-term rejection of iPSCderived neurons in non-human primates. Nat Commun 10,1-12.

Banki, N.M., Kopelnik, A., Dae, M.W., Miss, J., Tung, P., Lawton, M.T., Drew, B.J., Foster, E., Smith, W., Parmley, W.W., et al. (2005). Acute neurocardiogenic injury after subarachnoid hemorrhage. Circulation 112, 3314-3319.

Bar-Nur, O., Russ, H.A., Efrat, S., and Benvenisty, N. (2011). Epigenetic memory and preferential lineage-specific differentiation in induced pluripotent stem cells derived from human pancreatic islet beta cells. Cell Stem Cell 9, 17-23.

Bellin, M., Marchetto, M.C., Gage, F.H., and Mummery, C.L. (2012). Induced pluripotent stem cells: the new patient? Nat Rev Mol Cell Biol 13, 713-726. 
Bellin, M., Casini, S., Davis, R.P., D’Aniello, C., Haas, J., Ward-van Oostwaard, D., Tertoolen, L.G.J., Jung, C.B., Elliott, D.A., Welling, A., et al. (2013). Isogenic human pluripotent stem cell pairs reveal the role of a KCNH2 mutation in long-QT syndrome. EMBO J 32, 3161-3175.

Bers, D.M. (2002). Cardiac excitation-contraction coupling. Nature 415, 198-205.

Bharathi, K.S., Kulkarni, S., Sadananda, K.S., and Gurudatt, C.L. (2016). Takotsubo cardiomyopathy precipitated by negative pressure pulmonary oedema following total thyroidectomy. Indian J Anaesth 60, 202-205.

Blanc, C., Zeller, M., Cottin, Y., Daubail, B., Vialatte, A.-L., Giroud, M., and Béjot, Y. (2015). Takotsubo cardiomyopathy following acute aerebral events. Eur Neurol 74, 163-168.

Bonello, L., Com, O., Ait-Moktar, O., Théron, A., Moro, P.-J., Salem, A., Sbragia, P., and Paganelli, F. (2008). Ventricular arrhythmias during Tako-tsubo syndrome. Int J Cardiol 128, e50-53.

Borchert, T., Hübscher, D., Guessoum, C.I., Lam, T.-D.D., Ghadri, J.R., Schellinger, I.N., Tiburcy, M., Liaw, N.Y., Li, Y., Haas, J., et al. (2017). Catecholamine-dependent $\beta$-Adrenergic signaling in a pluripotent stem cell model of Takotsubo cardiomyopathy. J Am Coll Cardiol 70, 975-991.

Borchin, B., Chen, J., and Barberi, T. (2013). Derivation and FACS-mediated purification of $\mathrm{PAX} 3^{+} / \mathrm{PAX} 7+$ skeletal muscle precursors from human pluripotent stem cells. Stem Cell Reports 1, 620-631.

Brinjikji, W., El-Sayed, A.M., and Salka, S. (2012). In-hospital mortality among patients with takotsubo cardiomyopathy: A study of the National Inpatient Sample 2008 to 2009. Am Heart J 164, 215-221.

Brown, K.H., Trohman, R.G., and Madias, C. (2015). Arrhythmias in takotsubo cardiomyopathy. Card Electrophysiol Clin 7, 331-340.

Buganim, Y., Faddah, D.A., Cheng, A.W., Itskovich, E., Markoulaki, S., Ganz, K., Klemm, S.L., van Oudenaarden, A., and Jaenisch, R. (2012). Single-cell expression analyses during cellular reprogramming reveal an early stochastic and a late hierarchic phase. Cell 150, 1209-1222.

Bupha-Intr, T., and Wattanapermpool, J. (2006). Regulatory role of ovarian sex hormones in calcium uptake activity of cardiac sarcoplasmic reticulum. Am J of Physiol Heart and Circ Physiol 291, H1101-H1108.

Burkhardt, M.F., Martinez, F.J., Wright, S., Ramos, C., Volfson, D., Mason, M., Garnes, J., Dang, V., Lievers, J., Shoukat-Mumtaz, U., et al. (2013). A cellular model for sporadic ALS using patient-derived induced pluripotent stem cells. Mol Cell Neurosci 56, 355-364.

Burridge, P.W., Anderson, D., Priddle, H., Barbadillo Muñoz, M.D., Chamberlain, S., Allegrucci, C., Young, L.E., and Denning, C. (2007). Improved human embryonic stem cell embryoid body homogeneity and cardiomyocyte differentiation from a novel V-96 plate aggregation system highlights interline variability. Stem Cells 25, 929-938. 
Burridge, P.W., Matsa, E., Shukla, P., Lin, Z.C., Churko, J.M., Ebert, A.D., Lan, F., Diecke, S., Huber, B., Mordwinkin, N.M., et al. (2014). Chemically defined generation of human cardiomyocytes. Nat Methods 11, 855-860.

Burridge, P.W., Li, Y.F., Matsa, E., Wu, H., Ong, S.-G., Sharma, A., Holmström, A., Chang, A.C., Coronado, M.J., Ebert, A.D., et al. (2016). Human induced pluripotent stem cell-derived cardiomyocytes recapitulate the predilection of breast cancer patients to doxorubicin-induced cardiotoxicity. Nature Medicine 22, 547-556.

Cannavo, A., and Koch, W.J. (2017). Targeting $\beta_{3}$-adrenergic receptors in the heart: selective agonism and $\beta$-blockade. J Cardiovasc Pharmacol 69, 71-78.

Carvajal-Vergara, X., Sevilla, A., D’Souza, S.L., Ang, Y.-S., Schaniel, C., Lee, D.-F., Yang, L., Kaplan, A.D., Adler, E.D., Rozov, R., et al. (2010). Patient-specific induced pluripotent stem-cellderived models of LEOPARD syndrome. Nature 465, 808-812.

Chambers, I., Silva, J., Colby, D., Nichols, J., Nijmeijer, B., Robertson, M., Vrana, J., Jones, K., Grotewold, L., and Smith, A. (2007). Nanog safeguards pluripotency and mediates germline development. Nature 450, 1230-1234.

Chang, T., Zheng, W., Tsark, W., Bates, S., Huang, H., Lin, R.-J., and Yee, J.-K. (2011). Brief report: phenotypic rescue of induced pluripotent stem cell-derived motoneurons of a spinal muscular atrophy patient. Stem Cells 29, 2090-2093.

Cherian, J., Angelis, D., Filiberti, A., and Saperia, G. (2007). Can takotsubo cardiomyopathy be familial? Int J Cardiol 121, 74-75.

Chong, J.J.H., Yang, X., Don, C.W., Minami, E., Liu, Y.-W., Weyers, J.J., Mahoney, W.M., Van Biber, B., Cook, S.M., Palpant, N.J., et al. (2014). Human embryonic-stem-cell-derived cardiomyocytes regenerate non-human primate hearts. Nature 510, 273-277.

Christensen, T.E., Bang, L.E., Holmvang, L., Skovgaard, D.C., Oturai, D.B., Søholm, H., Thomsen, J.H., Andersson, H.B., Ghotbi, A.A., Ihlemann, N., et al. (2016). 123I-MIBG scintigraphy in the subacute state of Takotsubo cardiomyopathy. JACC: Cardiovasc Imaging 9 , 982-990.

Cyganek, L., Tiburcy, M., Sekeres, K., Gerstenberg, K., Bohnenberger, H., Lenz, C., Henze, S., Stauske, M., Salinas, G., Zimmermann, W.-H., et al. (2018). Deep phenotyping of human induced pluripotent stem cell-derived atrial and ventricular cardiomyocytes. JCI Insight 3, e99941.

Da Silveira Paulsen, B., De Moraes Maciel, R., Galina, A., Da Silveira, M.S., Souza, C.D.S., Drummond, H., Pozzatto, E.N., Junior, H.S., Chicaybam, L., Massuda, R., et al. (2012). Altered oxygen metabolism associated to neurogenesis of induced pluripotent stem cells derived from a schizophrenic patient. Cell Transplant 21, 1547-1559.

Davis, R.P., Casini, S., van den Berg, C.W., Hoekstra, M., Remme, C.A., Dambrot, C., Salvatori, D., Oostwaard, D.W., Wilde, A.A.M., Bezzina, C.R., et al. (2012). Cardiomyocytes derived from pluripotent stem cells recapitulate electrophysiological characteristics of an overlap syndrome of cardiac sodium channel disease. Circulation 125, 3079-3091. 
Delmas, C., Lairez, O., Mulin, E., Delmas, T., Boudou, N., Dumonteil, N., Biendel-Picquet, C., Roncalli, J., Elbaz, M., Galinier, M., et al. (2013). Anxiodepressive disorders and chronic psychological stress are associated with Tako-Tsubo cardiomyopathy. Circ J 77, 175-180.

Deshmukh, A., Kumar, G., Pant, S., Rihal, C., Murugiah, K., and Mehta, J.L. (2012). Prevalence of Takotsubo cardiomyopathy in the United States. Am Heart J 164, 66-71.e1.

Desmet, W.J.R., Adriaenssens, B.F.M., and Dens, J. a. Y. (2003). Apical ballooning of the left ventricle: first series in white patients. Heart 89, 1027-1031.

Di Giammartino, D.C., Kloetgen, A., Polyzos, A., Liu, Y., Kim, D., Murphy, D., Abuhashem, A., Cavaliere, P., Aronson, B., Shah, V., et al. (2019). KLF4 is involved in the organization and regulation of pluripotency-associated three-dimensional enhancer networks. Nat Cell Biol 21, 1179-1190.

Díaz-Díaz, C., Fernandez de Manuel, L., Jimenez-Carretero, D., Montoya, M.C., Clavería, C., and Torres, M. (2017). Pluripotency surveillance by Myc-driven competitive elimination of differentiating cells. Dev Cell 42, 585-599.e4.

Didié, M., Christalla, P., Rubart, M., Muppala, V., Döker, S., Unsöld, B., El-Armouche, A., Rau, T., Eschenhagen, T., Schwoerer, A.P., et al. (2013). Parthenogenetic stem cells for tissueengineered heart repair. J Clin Invest 123, 1285-1298.

Dressel, R., Nolte, J., Elsner, L., Novota, P., Guan, K., Streckfuss-Bömeke, K., Hasenfuss, G., Jaenisch, R., and Engel, W. (2010). Pluripotent stem cells are highly susceptible targets for syngeneic, allogeneic, and xenogeneic natural killer cells. FASEB J 24, 2164-2177.

Drosatos, K., Bharadwaj, K.G., Lymperopoulos, A., Ikeda, S., Khan, R., Hu, Y., Agarwal, R., Yu, S., Jiang, H., Steinberg, S.F., et al. (2011). Cardiomyocyte lipids impair $\beta$-adrenergic receptor function via PKC activation. Am J Physiol Endocrinol Metab 300, E489-499.

Dudek, J., Cheng, I.-F., Balleininger, M., Vaz, F.M., Streckfuss-Bömeke, K., Hübscher, D., Vukotic, M., Wanders, R.J.A., Rehling, P., and Guan, K. (2013). Cardiolipin deficiency affects respiratory chain function and organization in an induced pluripotent stem cell model of Barth syndrome. Stem Cell Res 11, 806-819.

Dupuis, M., van Rijckevorsel, K., Evrard, F., Dubuisson, N., Dupuis, F., and Van Robays, P. (2012). Takotsubo syndrome (TKS): a possible mechanism of sudden unexplained death in epilepsy (SUDEP). Seizure 21, 51-54.

Egawa, N., Kitaoka, S., Tsukita, K., Naitoh, M., Takahashi, K., Yamamoto, T., Adachi, F., Kondo, T., Okita, K., Asaka, I., et al. (2012). Drug screening for ALS using patient-specific induced pluripotent stem cells. Sci Transl Med 4, 145 ra104.

Eitel, I., von Knobelsdorff-Brenkenhoff, F., Bernhardt, P., Carbone, I., Muellerleile, K., Aldrovandi, A., Francone, M., Desch, S., Gutberlet, M., Strohm, O., et al. (2011). Clinical characteristics and cardiovascular magnetic resonance findings in stress (takotsubo) cardiomyopathy. JAMA 306, 277-286.

El Mahmoud, R., Mansencal, N., Pilliére, R., Leyer, F., Abbou, N., Michaud, P., Nallet, O., Digne, F., Lacombe, P., Cattan, S., et al. (2008). Prevalence and characteristics of left ventricular outflow tract obstruction in Tako-Tsubo syndrome. Am Heart J 156, 543-548. 
El-Battrawy, I., Zhao, Z., Lan, H., Schünemann, J.-D., Sattler, K., Buljubasic, F., Patocskai, B., Li, X., Yücel, G., Lang, S., et al. (2018). Estradiol protection against toxic effects of catecholamine on electrical properties in human-induced pluripotent stem cell derived cardiomyocytes. Int J Cardiol 254, 195-202.

Eskin, B.A., Snyder, D.L., Roberts, J., and Aloyo, V.J. (2003). Cardiac norepinephrine release: modulation by ovariectomy and estrogen. Exp Biol Med (Maywood) 228, 194-199.

Evans, M.J., and Kaufman, M.H. (1981). Establishment in culture of pluripotential cells from mouse embryos. Nature 292, 154-156.

Fertig, B.A., and Baillie, G.S. (2018). PDE4-Mediated cAMP signalling. J Cardiovasc Dev Dis 5.

Figtree, G.A., Bagnall, R.D., Abdulla, I., Buchholz, S., Galougahi, K.K., Yan, W., Tan, T., Neil, C., Horowitz, J.D., Semsarian, C., et al. (2013). No association of G-protein-coupled receptor kinase 5 or $\beta$-adrenergic receptor polymorphisms with Takotsubo cardiomyopathy in a large Australian cohort. Eur J Heart Fail 15, 730-733.

Fijalkowski, M., Fijalkowska, M., Nowak, R., and Rynkiewicz, A. (2013). Takotsubo cardiomyopathy in a male during a Euro 2012 football match. Clin Res Cardiol 102, 319-321.

Freund, C., Ward-van Oostwaard, D., Monshouwer-Kloots, J., van den Brink, S., van Rooijen, M., Xu, X., Zweigerdt, R., Mummery, C., and Passier, R. (2008). Insulin redirects differentiation from cardiogenic mesoderm and endoderm to neuroectoderm in differentiating human embryonic stem cells. Stem Cells 26, 724-733.

Fridericia, L.S. (1920). Die Systolendauer im Elektrokardiogramm bei normalen Menschen und bei Herzkranken. Acta Medica Scandinavica 53, 469-486.

Fusaki, N., Ban, H., Nishiyama, A., Saeki, K., and Hasegawa, M. (2009). Efficient induction of transgene-free human pluripotent stem cells using a vector based on Sendai virus, an RNA virus that does not integrate into the host genome. Proc Jpn Acad Ser B Phys Biol Sci 85, 348-362.

Galiuto, L., De Caterina, A.R., Porfidia, A., Paraggio, L., Barchetta, S., Locorotondo, G., Rebuzzi, A.G., and Crea, F. (2010). Reversible coronary microvascular dysfunction: a common pathogenetic mechanism in Apical Ballooning or Tako-Tsubo Syndrome. Eur Heart J 31, 13191327.

Gauthier, C., Leblais, V., Kobzik, L., Trochu, J.N., Khandoudi, N., Bril, A., Balligand, J.L., and Le Marec, $\mathrm{H}$. (1998). The negative inotropic effect of beta3-adrenoceptor stimulation is mediated by activation of a nitric oxide synthase pathway in human ventricle. J Clin Invest 102, 1377-1384.

Georgiadis, V., Stephanou, A., Townsend, P.A., and Jackson, T.R. (2015). MultiElec: a MATLAB based application for MEA data analysis. PLOS ONE 10, e0129389.

Geppert, G., Radke, P.W., Kurowski, V., Hunold, P., and Schunkert, H. (2010). Wespenstich, Adrenalingabe und akuter Thoraxschmerz: ein ungewöhnlicher Fall von stressinduzierter (TakoTsubo) Kardiomyopathie. Med Klin 105, 246-248.

Ghadri, J.-R., Wittstein, I.S., Prasad, A., Sharkey, S., Dote, K., Akashi, Y.J., Cammann, V.L., Crea, F., Galiuto, L., Desmet, W., et al. (2018a). International expert consensus document on 
Takotsubo Syndrome (Part I): clinical characteristics, diagnostic criteria, and pathophysiology. Eur Heart J 39, 2032-2046.

Ghadri, J.-R., Wittstein, I.S., Prasad, A., Sharkey, S., Dote, K., Akashi, Y.J., Cammann, V.L., Crea, F., Galiuto, L., Desmet, W., et al. (2018b). International expert consensus document on Takotsubo Syndrome (Part II): diagnostic workup, outcome, and management. Eur Heart J 39, 2047-2062.

Ghose, A., and Banerjee, S. (2019). Delayed onset Takotsubo syndrome following exercise ECG: a case report. European Heart Journal. Case Reports 3.

Ghosh, Z., Wilson, K.D., Wu, Y., Hu, S., Quertermous, T., and Wu, J.C. (2010). Persistent donor cell gene expression among human induced pluripotent stem cells contributes to differences with human embryonic stem cells. PLoS ONE 5, e8975.

Gianni, M., Dentali, F., Grandi, A.M., Sumner, G., Hiralal, R., and Lonn, E. (2006). Apical ballooning syndrome or takotsubo cardiomyopathy: a systematic review. Eur Heart J 27, 15231529.

Girardey, M., Jesel, L., Campia, U., Messas, N., Hess, S., Imperiale, A., Blondet, C., Trinh, A., OhImann, P., and Morel, O. (2016). Impact of malignancies in the early and late time course of Takotsubo cardiomyopathy. Circ J 80, 2192-2198.

Goh, P.A., Caxaria, S., Casper, C., Rosales, C., Warner, T.T., Coffey, P.J., and Nathwani, A.C. (2013). A systematic evaluation of integration free reprogramming methods for deriving clinically relevant patient specific induced pluripotent Stem (iPS) cells. PLoS One 8.

Guan, K., Nayernia, K., Maier, L.S., Wagner, S., Dressel, R., Lee, J.H., Nolte, J., Wolf, F., Li, M., Engel, W., et al. (2006). Pluripotency of spermatogonial stem cells from adult mouse testis. Nature 440, 1199-1203.

Guan, K., Wagner, S., Unsöld, B., Maier, L.S., Kaiser, D., Hemmerlein, B., Nayernia, K., Engel, W., and Hasenfuss, G. (2007). Generation of functional cardiomyocytes from adult mouse spermatogonial stem cells. Circ Res 100, 1615-1625.

Halevy, T., and Urbach, A. (2014). Comparing ESC and iPSC-based models for human genetic disorders. J Clin Med 3, 1146-1162.

Hanna, J., Wernig, M., Markoulaki, S., Sun, C.-W., Meissner, A., Cassady, J.P., Beard, C., Brambrink, T., Wu, L.-C., Townes, T.M., et al. (2007). Treatment of sickle cell anemia mouse model with iPS cells generated from autologous skin. Science 318, 1920-1923.

Hanses, U., Kleinsorge, M., Roos, L., Yigit, G., Li, Y., Barbarics, B., El-Battrawy, I., Lan, H., Tiburcy, M., Hindmarsh, R., et al. (2020). Intronic CRISPR Repair in a preclinical model of Noonan Syndrome-associated cardiomyopathy. Circulation 142, 1059-1076.

Haupt, L.P., Maus, A., Tiburcy, M., Köhne, S., Maurer, W., Tappu, R., Haas, J., Li, Y., Sasse, A., Santos, C.C.X., et al. (2020). Doxorubicin induces cardiotoxicity in a pluripotent stem cell model of aggressive B cell lymphoma cancer patients. BioRxiv 2020.04.15.042424. 
Heubach, J.F., Ravens, U., and Kaumann, A.J. (2004). Epinephrine activates both $\mathrm{G}_{\mathrm{s}}$ and $\mathrm{G}_{\mathrm{i}}$ pathways, but norepinephrine activates only the $\mathrm{G}_{\mathrm{s}}$ pathway through human beta2adrenoceptors overexpressed in mouse heart. Mol Pharmacol 65, 1313-1322.

Hiestand, T., Hänggi, J., Klein, C., Topka, M.S., Jaguszewski, M., Ghadri, J.R., Lüscher, T.F., Jäncke, L., and Templin, C. (2018). Takotsubo Syndrome associated with structural brain alterations of the limbic system. J Am Coll Cardiol 71, 809-811.

Holm, S. (2015). Biobanking human embryonic stem cell lines: policy, ethics and efficiency. Monash Bioeth Rev 33, 265-276.

Hou, P., Li, Y., Zhang, X., Liu, C., Guan, J., Li, H., Zhao, T., Ye, J., Yang, W., Liu, K., et al. (2013). Pluripotent stem cells induced from mouse somatic cells by small-molecule compounds. Science 341, 651-654.

Huangfu, D., Maehr, R., Guo, W., Eijkelenboom, A., Snitow, M., Chen, A.E., and Melton, D.A. (2008). Induction of pluripotent stem cells by defined factors is greatly improved by smallmolecule compounds. Nat Biotechnol 26, 795-797.

Hubbard, J.J., Sullivan, S.K., Mills, J.A., Hayes, B.J., Torok-Storb, B.J., and Ramakrishnan, A. (2014). Efficient iPS cell generation from blood using episomes and HDAC inhibitors. J Vis Exp e52009.

Hübscher, D., Rebs, S., Haupt, L., Borchert, T., Guessoum, C.I., Treu, F., Köhne, S., Maus, A., Hambrecht, M., Sossalla, S., et al. (2019). A high-throughput method as a diagnostic tool for HIV detection in patient-specific induced pluripotent stem cells generated by different reprogramming methods. Stem Cells Int 2019, 2181437.

Huebsch, N., Loskill, P., Deveshwar, N., Spencer, C.I., Judge, L.M., Mandegar, M.A., Fox, C.B., Mohamed, T.M.A., Ma, Z., Mathur, A., et al. (2016). Miniaturized iPS-cell-derived cardiac muscles for physiologically relevant drug response analyses. Sci Rep 6, 24726.

Hurst, R.T., Prasad, A., Askew, J.W., Sengupta, P.P., and Tajik, A.J. (2010). Takotsubo Cardiomyopathy: A unique cardiomyopathy with variable ventricular morphology. JACC: Cardiovascular Imaging 3, 641-649.

Icli, A., Akilli, H., Kayrak, M., Aribas, A., and Ozdemir, K. (2016). Short-term warfarin treatment for apical thrombus in a patient with Takotsubo cardiomyopathy. Cardiovasc J Afr 27, e12-e14.

Ikutomi, M., Yamasaki, M., Matsusita, M., Watari, Y., Arashi, H., Endo, G., Yamaguchi, J., and Ohnishi, S. (2014). Takotsubo cardiomyopathy in siblings. Heart Vessels 29, 119-122.

Inamasu, J., Ganaha, T., Nakae, S., Ohmi, T., Wakako, A., Tanaka, R., Kuwahara, K., Kogame, H., Kawazoe, Y., Kumai, T., et al. (2016). Therapeutic outcomes for patients with aneurysmal subarachnoid hemorrhage complicated by Takotsubo cardiomyopathy. Acta Neurochir 158, 885-893.

Isogai, S., Yamamoto, N., Hiramatsu, N., Goto, Y., Hayashi, M., Kondo, M., and Imaizumi, K. (2018). Preparation of induced pluripotent stem cells using human peripheral blood monocytes. Cell Reprogram 20, 347-355. 
Isogai, T., Matsui, H., Tanaka, H., Fushimi, K., and Yasunaga, H. (2016). Early $\beta$-blocker use and in-hospital mortality in patients with Takotsubo cardiomyopathy. Heart 102, 1029-1035.

Izumi, Y., Okatani, H., Shiota, M., Nakao, T., Ise, R., Kito, G., Miura, K., and Iwao, H. (2009). Effects of metoprolol on epinephrine-induced takotsubo-like left ventricular dysfunction in nonhuman primates. Hypertens Res 32, 339-346.

Jacków, J., Guo, Z., Hansen, C., Abaci, H.E., Doucet, Y.S., Shin, J.U., Hayashi, R., DeLorenzo, D., Kabata, Y., Shinkuma, S., et al. (2019). CRISPR/Cas9-based targeted genome editing for correction of recessive dystrophic epidermolysis bullosa using iPS cells. Proc Natl Acad Sci U S A 116, 26846-26852.

Jaguszewski, M., Fijalkowski, M., Nowak, R., Czapiewski, P., Ghadri, J.-R., Templin, C., and Rynkiewicz, A. (2012). Ventricular rupture in Takotsubo cardiomyopathy. Eur Heart J 33, 1027.

Jaguszewski, M., Osipova, J., Ghadri, J.-R., Napp, L.C., Widera, C., Franke, J., Fijalkowski, M., Nowak, R., Fijalkowska, M., Volkmann, I., et al. (2014). A signature of circulating microRNAs differentiates takotsubo cardiomyopathy from acute myocardial infarction. Eur Heart J 35, 9991006.

Jiang, X., Yang, Z., and Dong, M. (2020). Cardiac repair in a murine model of myocardial infarction with human induced pluripotent stem cell-derived cardiomyocytes. Stem Cell Res Ther $11,297$.

Jodalen, H., Lie, R., and Rotevatn, S. (1982). Effect of isoproterenol on lipid accumulation in myocardial cells. Research in Experimental Medicine. Res Exp Med (Berl) 181, 239-244.

Jones, V.C., Atkinson-Dell, R., Verkhratsky, A., and Mohamet, L. (2017). Aberrant iPSC-derived human astrocytes in Alzheimer's disease. Cell Death Dis 8, e2696.

Kattman, S.J., Witty, A.D., Gagliardi, M., Dubois, N.C., Niapour, M., Hotta, A., Ellis, J., and Keller, G. (2011). Stage-specific optimization of activin/nodal and BMP signaling promotes cardiac differentiation of mouse and human pluripotent stem cell lines. Cell Stem Cell 8, $228-$ 240.

Kawada, J., Kaneda, S., Kirihara, T., Maroof, A., Levi, T., Eggan, K., Fujii, T., and Ikeuchi, Y. (2017). Generation of a motor nerve organoid with human stem cell-derived neurons. Stem Cell Reports 9, 1441-1449.

Kennedy, M., Awong, G., Sturgeon, C.M., Ditadi, A., LaMotte-Mohs, R., Zúñiga-Pflücker, J.C., and Keller, G. (2012). T lymphocyte potential marks the emergence of definitive hematopoietic progenitors in human pluripotent stem cell differentiation cultures. Cell Rep 2, 1722-1735.

Kiechle, M. (2008). [Human embryonic stem cell research in Germany. The scientific reviewing of applications for the import and use of human embryonic stem cells]. Bundesgesundheitsblatt Gesundheitsforschung Gesundheitsschutz 51, 961-964.

Kikuchi, T., Morizane, A., Doi, D., Magotani, H., Onoe, H., Hayashi, T., Mizuma, H., Takara, S., Takahashi, R., Inoue, H., et al. (2017). Human iPS cell-derived dopaminergic neurons function in a primate Parkinson's disease model. Nature 548, 592-596. 
Kim, D., Kim, C.-H., Moon, J.-I., Chung, Y.-G., Chang, M.-Y., Han, B.-S., Ko, S., Yang, E., Cha, K.Y., Lanza, R., et al. (2009). Generation of human induced pluripotent stem cells by direct delivery of reprogramming proteins. Cell Stem Cell 4, 472-476.

Kim, S., Yu, A., Filippone, L.A., Kolansky, D.M., and Raina, A. (2010). Inverted-Takotsubo pattern cardiomyopathy secondary to pheochromocytoma: A clinical case and literature review. Clin Cardiol 33, 200-205.

Kiowski, W., Lüscher, T.F., Linder, L., and Bühler, F.R. (1991). Endothelin-1-induced vasoconstriction in humans. Reversal by calcium channel blockade but not by nitrovasodilators or endothelium-derived relaxing factor. Circulation 83, 469-475.

Kogut, I., McCarthy, S.M., Pavlova, M., Astling, D.P., Chen, X., Jakimenko, A., Jones, K.L., Getahun, A., Cambier, J.C., Pasmooij, A.M.G., et al. (2018). High-efficiency RNA-based reprogramming of human primary fibroblasts. Nat Commun 9, 745.

Kolanowski, T.J., Antos, C.L., and Guan, K. (2017). Making human cardiomyocytes up to date: Derivation, maturation state and perspectives. Int J Cardiol 241, 379-386.

Komesaroff, P.A., Esler, M.D., and Sudhir, K. (1999). Estrogen supplementation attenuates glucocorticoid and catecholamine responses to mental stress in perimenopausal women. J Clin Endocrinol Metab 84, 606-610.

Krupnick, J.G., and Benovic, J.L. (1998). The role of receptor kinases and arrestins in G proteincoupled receptor regulation. Annu Rev Pharmacol Toxicol 38, 289-319.

Kujala, K., Paavola, J., Lahti, A., Larsson, K., Pekkanen-Mattila, M., Viitasalo, M., Lahtinen, A.M., Toivonen, L., Kontula, K., Swan, H., et al. (2012). Cell model of catecholaminergic polymorphic ventricular tachycardia reveals early and delayed afterdepolarizations. PLoS One 7.

Kumar, G., Holmes, D.R., and Prasad, A. (2010). "Familial” apical ballooning syndrome (Takotsubo cardiomyopathy). Int J Cardiol 144, 444-445.

Kume, T., Kawamoto, T., Okura, H., Toyota, E., Neishi, Y., Watanabe, N., Hayashida, A., Okahashi, N., Yoshimura, Y., Saito, K., et al. (2008). Local release of catecholamines from the hearts of patients with tako-tsubo-like left ventricular dysfunction. Circ J 72, 106-108.

Lacey, C.J., Doudney, K., Bridgman, P.G., George, P.M., Mulder, R.T., Zarifeh, J.J., Kimber, B., Cadzow, M.J., Black, M.A., Merriman, T.R., et al. (2018). Copy number variants implicate cardiac function and development pathways in earthquake-induced stress cardiomyopathy. Sci Rep 8, 7548.

Lacy, C.R., Contrada, R.J., Robbins, M.L., Tannenbaum, A.K., Moreyra, A.E., Chelton, S., and Kostis, J.B. (1995). Coronary vasoconstriction induced by mental stress (simulated public speaking). Am J Cardiol 75, 503-505.

Lancaster, M.A., Renner, M., Martin, C.-A., Wenzel, D., Bicknell, L.S., Hurles, M.E., Homfray, T., Penninger, J.M., Jackson, A.P., and Knoblich, J.A. (2013). Cerebral organoids model human brain development and microcephaly. Nature 501, 373-379. 
Lee, M. (2020). Time course of functional recovery in Takotsubo (Stress) Cardiomyopathy: A serial speckle tracking echocardiography and electrocardiography study. J Cardiovasc Imaging $28,50-60$.

Lee, G., Papapetrou, E.P., Kim, H., Chambers, S.M., Tomishima, M.J., Fasano, C.A., Ganat, Y.M., Menon, J., Shimizu, F., Viale, A., et al. (2009). Modelling pathogenesis and treatment of familial dysautonomia using patient-specific iPSCs. Nature 461, 402-406.

Lee, J., Sutani, A., Kaneko, R., Takeuchi, J., Sasano, T., Kohda, T., Ihara, K., Takahashi, K., Yamazoe, M., Morio, T., et al. (2020). In vitro generation of functional murine heart organoids via FGF4 and extracellular matrix. Nat Commun 11, 4283.

Li, M., and Belmonte, J.C.I. (2017). Ground rules of the pluripotency gene regulatory network. Nat Rev Genet 18, 180-191.

Li, H.L., Fujimoto, N., Sasakawa, N., Shirai, S., Ohkame, T., Sakuma, T., Tanaka, M., Amano, N., Watanabe, A., Sakurai, H., et al. (2014). Precise correction of the Dystrophin gene in Duchenne muscular dystrophy patient induced pluripotent stem cells by TALEN and CRISPRCas9. Stem Cell Reports 4, 143-154.

Li, H.Y., Bian, J.S., Kwan, Y.W., and Wong, T.M. (2000a). Enhanced responses to 17betaestradiol in rat hearts treated with isoproterenol: involvement of a cyclic AMP-dependent pathway. J Pharmacol Exp Ther 293, 592-598.

Li, L., Desantiago, J., Chu, G., Kranias, E.G., and Bers, D.M. (2000b). Phosphorylation of phospholamban and troponin I in beta-adrenergic-induced acceleration of cardiac relaxation. Am J Physiol Heart Circ Physiol 278, H769-779.

Li, Y., Wang, H., Muffat, J., Cheng, A.W., Orlando, D.A., Lovén, J., Kwok, S.-M., Feldman, D.A., Bateup, H.S., Gao, Q., et al. (2013). Global transcriptional and translational repression in human-embryonic-stem-cell-derived Rett syndrome neurons. Cell Stem Cell 13, 446-458.

Liang, G., Taranova, O., Xia, K., and Zhang, Y. (2010). Butyrate promotes induced pluripotent stem cell generation. J Biol Chem 285, 25516-25521.

Liggett, S.B., Cresci, S., Kelly, R.J., Syed, F.M., Matkovich, S.J., Hahn, H.S., Diwan, A., Martini, J.S., Sparks, L., Parekh, R.R., et al. (2008). A GRK5 polymorphism that inhibits beta-adrenergic receptor signaling is protective in heart failure. Nat Med 14, 510-517.

Liu, H., Kim, Y., Sharkis, S., Marchionni, L., and Jang, Y.-Y. (2011). In vivo liver regeneration potential of human induced pluripotent stem cells from diverse origins. Sci Transl Med 3, 82ra39.

Loh, Y.-H., Agarwal, S., Park, I.-H., Urbach, A., Huo, H., Heffner, G.C., Kim, K., Miller, J.D., Ng, K., and Daley, G.Q. (2009). Generation of induced pluripotent stem cells from human blood. Blood 113, 5476-5479.

Lu, B., and Palacino, J. (2013). A novel human embryonic stem cell-derived Huntington's disease neuronal model exhibits mutant huntingtin ( $\mathrm{mHTT}$ ) aggregates and soluble $\mathrm{mHTT}$ dependent neurodegeneration. FASEB J 27, 1820-1829. 
Lu, H.F., Lim, S.-X., Leong, M.F., Narayanan, K., Toh, R.P.K., Gao, S., and Wan, A.C.A. (2012). Efficient neuronal differentiation and maturation of human pluripotent stem cells encapsulated in 3D microfibrous scaffolds. Biomaterials 33, 9179-9187.

Lundy, S.D., Zhu, W.-Z., Regnier, M., and Laflamme, M.A. (2013). Structural and functional maturation of cardiomyocytes derived from human pluripotent stem cells. Stem Cells Dev 22, 1991-2002.

Ma, D., Wei, H., Lu, J., Ho, S., Zhang, G., Sun, X., Oh, Y., Tan, S.H., Ng, M.L., Shim, W., et al. (2013). Generation of patient-specific induced pluripotent stem cell-derived cardiomyocytes as a cellular model of arrhythmogenic right ventricular cardiomyopathy. Eur Heart J 34, 1122-1133.

Ma, H., Morey, R., O’Neil, R.C., He, Y., Daughtry, B., Schultz, M.D., Hariharan, M., Nery, J.R., Castanon, R., Sabatini, K., et al. (2014). Abnormalities in human pluripotent cells due to reprogramming mechanisms. Nature 511, 177-183.

Mai, Q., Yu, Y., Li, T., Wang, L., Chen, M., Huang, S., Zhou, C., and Zhou, Q. (2007). Derivation of human embryonic stem cell lines from parthenogenetic blastocysts. Cell Res 17, 1008-1019.

Mandal, P.K., and Rossi, D.J. (2013). Reprogramming human fibroblasts to pluripotency using modified mRNA. Nat Protoc 8, 568-582.

Manea, M., Comsa, M., Minca, A., Dragos, D., and Popa, C. (2015). Brain-heart axis - Review Article. J Med Life 8, 266-271.

Masui, S., Nakatake, Y., Toyooka, Y., Shimosato, D., Yagi, R., Takahashi, K., Okochi, H., Okuda, A., Matoba, R., Sharov, A.A., et al. (2007). Pluripotency governed by Sox2 via regulation of Oct3/4 expression in mouse embryonic stem cells. Nat Cell Biol 9, 625-635.

Masumoto, H., Nakane, T., Tinney, J.P., Yuan, F., Ye, F., Kowalski, W.J., Minakata, K., Sakata, R., Yamashita, J.K., and Keller, B.B. (2016). The myocardial regenerative potential of threedimensional engineered cardiac tissues composed of multiple human iPS cell-derived cardiovascular cell lineages. Sci Rep 6, 1-10.

Matsa, E., Rajamohan, D., Dick, E., Young, L., Mellor, I., Staniforth, A., and Denning, C. (2011). Drug evaluation in cardiomyocytes derived from human induced pluripotent stem cells carrying a long QT syndrome type 2 mutation. Eur Heart J 32, 952-962.

Mattsson, E., Saliba-Gustafsson, P., Ehrenborg, E., and Tornvall, P. (2018). Lack of genetic susceptibility in takotsubo cardiomyopathy: a case-control study. BMC Med Genet 19, 39.

Maxwell, M.A., Cleasby, M.E., Harding, A., Stark, A., Cooney, G.J., and Muscat, G.E.O. (2005). Nur77 regulates lipolysis in skeletal muscle cells. Evidence for cross-talk between the betaadrenergic and an orphan nuclear hormone receptor pathway. J Biol Chem 280, 12573-12584.

Medzikovic, L., Schumacher, C.A., Verkerk, A.O., van Deel, E.D., Wolswinkel, R., van der Made, I., Bleeker, N., Cakici, D., van den Hoogenhof, M.M.G., Meggouh, F., et al. (2015). Orphan nuclear receptor Nur77 affects cardiomyocyte calcium homeostasis and adverse cardiac remodelling. Sci Rep 5, 15404.

Menasché, P., Vanneaux, V., Hagège, A., Bel, A., Cholley, B., Cacciapuoti, I., Parouchev, A., Benhamouda, N., Tachdjian, G., Tosca, L., et al. (2015). Human embryonic stem cell-derived 
cardiac progenitors for severe heart failure treatment: first clinical case report. Eur Heart J 36, 2011-2017.

Menasché, P., Vanneaux, V., Hagège, A., Bel, A., Cholley, B., Parouchev, A., Cacciapuoti, I., AlDaccak, R., Benhamouda, N., Blons, H., et al. (2018). Transplantation of human embryonic stem cell-derived cardiovascular progenitors for severe ischemic left ventricular dysfunction. JACC 71, 429-438.

Moretti, A., Bellin, M., Welling, A., Jung, C.B., Lam, J.T., Bott-Flügel, L., Dorn, T., Goedel, A., Höhnke, C., Hofmann, F., et al. (2010). Patient-specific induced pluripotent stem-cell models for Long-QT syndrome. N Engl J Med 363, 1397-1409.

Morizane, A., Kikuchi, T., Hayashi, T., Mizuma, H., Takara, S., Doi, H., Mawatari, A., Glasser, M.F., Shiina, T., Ishigaki, H., et al. (2017). MHC matching improves engraftment of iPSC-derived neurons in non-human primates. Nat Commun 8, 1-12.

Mummery, C.L., Zhang, J., Ng, E.S., Elliott, D.A., Elefanty, A.G., and Kamp, T.J. (2012). Differentiation of human ES and iPS cells to cardiomyocytes: A Methods Overview. Circ Res 111, 344-358.

Mummery, C., Ward-van Oostwaard, D., Doevendans, P., Spijker, R., van den Brink, S., Hassink, R., van der Heyden, M., Opthof, T., Pera, M., de la Riviere Aart, B., et al. (2003). Differentiation of human embryonic stem cells to Cardiomyocytes. Circulation 107, 2733-2740.

Nakano, T., Onoue, K., Nakada, Y., Nakagawa, H., Kumazawa, T., Ueda, T., Nishida, T., Soeda, T., Okayama, S., Watanabe, M., et al. (2018). Alteration of $\beta$-Adrenoceptor signaling in left ventricle of acute phase Takotsubo Syndrome: a human study. Sci Rep 8, 12731.

Nef, H.M., Möllmann, H., Hilpert, P., Troidl, C., Voss, S., Rolf, A., Behrens, C.B., Weber, M., Hamm, C.W., and Elsässer, A. (2009a). Activated cell survival cascade protects cardiomyocytes from cell death in Tako-Tsubo cardiomyopathy. Eur $\mathrm{J}$ of Heart Fail 11, 758-764.

Nef, H.M., Möllmann, H., Troidl, C., Kostin, S., Voss, S., Hilpert, P., Behrens, C.B., Rolf, A., Rixe, J., Weber, M., et al. (2009b). Abnormalities in intracellular $\mathrm{Ca}^{2+}$ regulation contribute to the pathomechanism of Tako-Tsubo cardiomyopathy. Eur Heart J 30, 2155-2164.

Nichols, J., Zevnik, B., Anastassiadis, K., Niwa, H., Klewe-Nebenius, D., Chambers, I., Schöler, H., and Smith, A. (1998). Formation of pluripotent stem cells in the mammalian embryo depends on the POU transcription factor Oct4. Cell 95, 379-391.

Noguchi, H., Miyagi-Shiohira, C., and Nakashima, Y. (2018). Induced tissue-specific stem cells and epigenetic memory in induced pluripotent stem cells. Int J Mol Sci 19.

O'Connor, M.D., Kardel, M.D., losfina, I., Youssef, D., Lu, M., Li, M.M., Vercauteren, S., Nagy, A., and Eaves, C.J. (2008). Alkaline phosphatase-positive colony formation is a sensitive, specific, and quantitative indicator of undifferentiated human embryonic stem cells. Stem Cells 26, 1109-1116.

Okita, K., Matsumura, Y., Sato, Y., Okada, A., Morizane, A., Okamoto, S., Hong, H., Nakagawa, M., Tanabe, K., Tezuka, K., et al. (2011). A more efficient method to generate integration-free human iPS cells. Nat Methods 8, 409-412. 
Okita, K., Yamakawa, T., Matsumura, Y., Sato, Y., Amano, N., Watanabe, A., Goshima, N., and Yamanaka, S. (2013). An efficient nonviral method to generate integration-free human-induced pluripotent stem cells from cord blood and peripheral blood cells. Stem Cells 31, 458-466.

Ou, Z., Niu, X., He, W., Chen, Y., Song, B., Xian, Y., Fan, D., Tang, D., and Sun, X. (2016). The combination of CRISPR/Cas9 and iPSC technologies in the gene therapy of human $\beta$ thalassemia in mice. Sci Rep 6, 1-13.

Parikh, S.S., Blackwell, D.J., Gomez-Hurtado, N., Frisk, M., Wang, L., Kim, K., Dahl, C.P., Fiane, A., Tønnessen, T., Kryshtal, D.O., et al. (2017). Thyroid and glucocorticoid hormones promote functional T-Tubule development in human-induced pluripotent stem cell-derived cardiomyocytes. Circ Res 121, 1323-1330.

Park, S.-J., Kim, R.Y., Park, B.-W., Lee, S., Choi, S.W., Park, J.-H., Choi, J.J., Kim, S.-W., Jang, J., Cho, D.-W., et al. (2019). Dual stem cell therapy synergistically improves cardiac function and vascular regeneration following myocardial infarction. Nat Commun 10, 3123.

Paulsen, B. da S., Cardoso, S.C., Stelling, M.P., Cadilhe, D.V., and Rehen, S.K. (2014). Valproate reverts zinc and potassium imbalance in schizophrenia-derived reprogrammed cells. Schizophr Res 154, 30-35.

Paur, H., Wright, P.T., Sikkel, M.B., Tranter, M.H., Mansfield, C., O'Gara, P., Stuckey, D.J., Nikolaev, V.O., Diakonov, I., Pannell, L., et al. (2012). High levels of circulating epinephrine trigger apical cardiodepression in a $\beta_{2}$-adrenergic receptor/Gi-dependent manner: a new model of Takotsubo cardiomyopathy. Circulation 126, 697-706.

Peters, S., and Klein, H.U. (2012). WCD LifeVest: risk stratification in a case of Tako-Tsubo cardiomyopathy with QT interval prolongation. Herz 37, 219-221.

Picht, E., Zima, A.V., Blatter, L.A., and Bers, D.M. (2007). SparkMaster: automated calcium spark analysis with ImageJ. Am J Physiol Cell Physiol 293, C1073-1081.

Pierpont, G.L., DeMaster, E.G., and Cohn, J.N. (1984). Regional differences in adrenergic function within the left ventricle. Am J Physiol 246, H824-829.

Pison, L., De Vusser, P., and Mullens, W. (2004). Apical ballooning in relatives. Heart 90, e67.

Prasad, A., Lerman, A., and Rihal, C.S. (2008). Apical ballooning syndrome (Tako-Tsubo or stress cardiomyopathy): a mimic of acute myocardial infarction. Am Heart J 155, 408-417.

Qu, X., Liu, T., Song, K., Li, X., and Ge, D. (2012). Induced pluripotent stem cells generated from human adipose-derived stem cells using a non-viral polycistronic plasmid in feeder-free conditions. PLoS ONE 7, e48161.

Rand, T.A., Sutou, K., Tanabe, K., Jeong, D., Nomura, M., Kitaoka, F., Tomoda, E., Narita, M., Nakamura, M., Nakamura, M., et al. (2018). MYC releases early reprogrammed human cells from proliferation pause via retinoblastoma protein inhibition. Cell Rep 23, 361-375.

Ranki, H.J., Budas, G.R., Crawford, R.M., and Jovanović, A. (2001). Gender-specific difference in cardiac ATP-sensitive K(+) channels. J Am Coll Cardiol 38, 906-915. 
Rashid, S.T., Corbineau, S., Hannan, N., Marciniak, S.J., Miranda, E., Alexander, G., HuangDoran, I., Griffin, J., Ahrlund-Richter, L., Skepper, J., et al. (2010). Modeling inherited metabolic disorders of the liver using human induced pluripotent stem cells. J Clin Invest 120, 3127-3136.

Ren, Y., Jiang, H., Hu, Z., Fan, K., Wang, J., Janoschka, S., Wang, X., Ge, S., and Feng, J. (2015). Parkin mutations reduce the complexity of neuronal processes in iPSC-derived human neurons. Stem Cells 33, 68-78.

Revazova, E. s., Turovets, N. a., Kochetkova, O. d., Kindarova, L. b., Kuzmichev, L. n., Janus, J. d., and Pryzhkova, M. v. (2007). Patient-specific stem cell lines derived from human parthenogenetic blastocysts. Cloning Stem Cells 9, 432-449.

Rodriguez-Fraticelli, A.E., Wolock, S.L., Weinreb, C.S., Panero, R., Patel, S.H., Jankovic, M., Sun, J., Calogero, R.A., Klein, A.M., and Camargo, F.D. (2018). Clonal analysis of lineage fate in native haematopoiesis. Nature 553, 212-216.

Ruan, J.-L., Tulloch, N.L., Razumova, M.V., Saiget, M., Muskheli, V., Pabon, L., Reinecke, H., Regnier, M., and Murry, C.E. (2016). Mechanical stress conditioning and electrical stimulation promote contractility and force maturation of induced pluripotent stem cell-derived human cardiac tissue. Circulation 134, 1557-1567.

Ruzo, A., Croft, G.F., Metzger, J.J., Galgoczi, S., Gerber, L.J., Pellegrini, C., Wang, H., Fenner, M., Tse, S., Marks, A., et al. (2018). Chromosomal instability during neurogenesis in Huntington's disease. Development 145.

Sader, M. (2002). Endothelial function, vascular reactivity and gender differences in the cardiovascular system. Cardiovasc Res 53, 597-604.

Sagi, I., Chia, G., Golan-Lev, T., Peretz, M., Weissbein, U., Sui, L., Sauer, M.V., Yanuka, O., Egli, D., and Benvenisty, N. (2016). Derivation and differentiation of haploid human embryonic stem cells. Nature 532, 107-111.

Sagie, S., Ellran, E., Katzir, H., Shaked, R., Yehezkel, S., Laevsky, I., Ghanayim, A., Geiger, D., Tzukerman, M., and Selig, S. (2014). Induced pluripotent stem cells as a model for telomeric abnormalities in ICF type I syndrome. Hum Mol Genet 23, 3629-3640.

Sances, S., Bruijn, L.I., Chandran, S., Eggan, K., Ho, R., Klim, J.R., Livesey, M.R., Lowry, E., Macklis, J.D., Rushton, D., et al. (2016). Modeling ALS with motor neurons derived from human induced pluripotent stem cells. Nat Neurosci 19, 542-553.

Santoro, F., leva, R., Ferraretti, A., lenco, V., Carpagnano, G., Lodispoto, M., Di Biase, L., Di Biase, M., and Brunetti, N.D. (2013). Safety and feasibility of levosimendan administration in takotsubo cardiomyopathy: a case series. Cardiovasc Ther 31, e133-137.

Sato, H. (1990). Tako-tsubo-like left ventricular dysfunction due to multivessel coronary spasm. Clinical Aspects of Myocardial Injury: From Ischemia to Heart Failure 56-64.

Sattler, K., El-Battrawy, I., Lang, S., Zhou, X., Schramm, K., Tülümen, E., Kronbach, F., Röger, S., Behnes, M., Kuschyk, J., et al. (2017). Prevalence of cancer in Takotsubo cardiomyopathy: Short and long-term outcome. Int J Cardiol 238, 159-165. 
Sattler, K., El-Battrawy, I., Gietzen, T., Lang, S., Zhou, X., Borggrefe, M., and Akin, I. (2018). Long term outcome of patients suffering from cancer and Takotsubo syndrome or myocardial infarction. QJM 111, 473-481.

Scheitz, J.F., Mochmann, H.C., Witzenbichler, B., Fiebach, J.B., Audebert, H.J., and Nolte, C.H. (2012). Takotsubo cardiomyopathy following ischemic stroke: a cause of troponin elevation. J Neurol 259, 188-190.

Schmidt, K.H., Herholz, T., Rodeck, J., Abegunewardene, N., Kreitner, K.-F., and Münzel, T. (2017). Pheochromocytoma triggers takotsubo syndrome complicated by cerebral and peripheral embolic events. Eur Heart J 38, 1522-1523.

Scuderi, G.J., and Butcher, J. (2017). Naturally engineered maturation of cardiomyocytes. Front Cell Dev Biol 5, 50.

Shaikh, N., Sardar, M., Jacob, A., Alagusundaramoorthy, S.S., Eng, M., Checton, J., and Shah, A. (2018). Possible predictive factors for recovery of left ventricular systolic function in Takotsubo cardiomyopathy. Intractable Rare Dis Res 7, 100-105.

Shao, Y., Redfors, B., Ståhlman, M., Täng, M.S., Miljanovic, A., Möllmann, H., Troidl, C., Szardien, S., Hamm, C., Nef, H., et al. (2013). A mouse model reveals an important role for catecholamine-induced lipotoxicity in the pathogenesis of stress-induced cardiomyopathy. Eur $\mathrm{J}$ Heart Fail 15, 9-22.

Sharkey, S.W., Maron, B.J., Nelson, P., Parpart, M., Maron, M.S., and Bristow, M.R. (2009). Adrenergic receptor polymorphisms in patients with stress (tako-tsubo) cardiomyopathy. J Cardiol 53, 53-57.

Sharkey, S.W., Windenburg, D.C., Lesser, J.R., Maron, M.S., Hauser, R.G., Lesser, J.N., Haas, T.S., Hodges, J.S., and Maron, B.J. (2010). Natural history and expansive clinical profile of stress (tako-tsubo) cardiomyopathy. J Am Coll Cardiol 55, 333-341.

Sharkey, S.W., McAllister, N., Dassenko, D., Lin, D., Han, K., and Maron, B.J. (2015). Evidence that high catecholamine levels produced by pheochromocytoma may be responsible for TakoTsubo cardiomyopathy. Am J Cardiol 115, 1615-1618.

Sharma, A., Burridge, P.W., McKeithan, W.L., Serrano, R., Shukla, P., Sayed, N., Churko, J.M., Kitani, T., Wu, H., Holmström, A., et al. (2017). High-throughput screening of tyrosine kinase inhibitor cardiotoxicity with human induced pluripotent stem cells. Sci Transl Med 9.

Shiba, Y., Gomibuchi, T., Seto, T., Wada, Y., Ichimura, H., Tanaka, Y., Ogasawara, T., Okada, K., Shiba, N., Sakamoto, K., et al. (2016). Allogeneic transplantation of iPS cell-derived cardiomyocytes regenerates primate hearts. Nature 538, 388-391.

Spadari, R.C., Cavadas, C., de Carvalho, A.E.T.S., Ortolani, D., de Moura, A.L., and Vassalo, P.F. (2018). Role of beta-adrenergic receptors and Sirtuin signaling in the heart during aging, heart failure, and adaptation to stress. Cell Mol Neurobiol 38, 109-120.

Spinelli, L., Trimarco, V., Di Marino, S., Marino, M., Iaccarino, G., and Trimarco, B. (2010). L41Q polymorphism of the $\mathrm{G}$ protein coupled receptor kinase 5 is associated with left ventricular apical ballooning syndrome. Eur J Heart Fail 12, 13-16. 
Steinfath, M., Lavicky, J., Schmitz, W., Scholz, H., Döring, V., and Kalmár, P. (1992). Regional distribution of beta 1 - and beta 2-adrenoceptors in the failing and nonfailing human heart. Eur $\mathrm{J}$ Clin Pharmacol 42, 607-611.

Stiermaier, T., Eitel, C., Denef, S., Desch, S., Schuler, G., Thiele, H., and Eitel, I. (2015).

Prevalence and clinical significance of life-threatening arrhythmias in

Takotsubo Cardiomyopathy. J Am Coll Cardiol 65, 2148-2150.

Stiermaier, T., Moeller, C., Oehler, K., Desch, S., Graf, T., Eitel, C., Vonthein, R., Schuler, G., Thiele, H., and Eitel, I. (2016). Long-term excess mortality in takotsubo cardiomyopathy: predictors, causes and clinical consequences. Eur J Heart Fail 18, 650-656.

Stöllberger, C., and Finsterer, J. (2019). Delayed onset of Takotsubo syndrome after epileptic seizure. Neurol Neurochir Pol 53, 95-97.

Streckfuss-Bömeke, K., Vlasov, A., Hülsmann, S., Yin, D., Nayernia, K., Engel, W., Hasenfuss, G., and Guan, K. (2009). Generation of functional neurons and glia from multipotent adult mouse germ-line stem cells. Stem Cell Res 2, 139-154.

Streckfuss-Bömeke, K., Wolf, F., Azizian, A., Stauske, M., Tiburcy, M., Wagner, S., Hübscher, D., Dressel, R., Chen, S., Jende, J., et al. (2013). Comparative study of human-induced pluripotent stem cells derived from bone marrow cells, hair keratinocytes, and skin fibroblasts. Eur Heart J 34, 2618-2629.

Streckfuss-Bömeke, K., Tiburcy, M., Fomin, A., Luo, X., Li, W., Fischer, C., Özcelik, C., Perrot, A., Sossalla, S., Haas, J., et al. (2017). Severe DCM phenotype of patient harboring RBM20 mutation S635A can be modeled by patient-specific induced pluripotent stem cell-derived cardiomyocytes. J Mol Cell Cardiol 113, 9-21.

Sugii, S., Kida, Y., Berggren, W.T., and Evans, R.M. (2011). Feeder-dependent and feederindependent iPS cell derivation from human and mouse adipose stem cells. Nat Protoc 6, 346358.

Sugimoto, K., Inamasu, J., Hirose, Y., Kato, Y., Ito, K., Iwase, M., Sugimoto, K., Watanabe, E., Takahashi, A., and Ozaki, Y. (2012). The role of norepinephrine and estradiol in the pathogenesis of cardiac wall motion abnormality associated with subarachnoid hemorrhage. Stroke 43, 1897-1903.

Summers, M.R., Lennon, R.J., and Prasad, A. (2010). Pre-morbid psychiatric and cardiovascular diseases in apical ballooning syndrome (Tako-Tsubo/Stress-Induced Cardiomyopathy): Potential pre-disposing factors? J Am Coll Cardiol 55, 700-701.

Sun, N., Yazawa, M., Liu, J., Han, L., Sanchez-Freire, V., Abilez, O.J., Navarrete, E.G., Hu, S., Wang, L., Lee, A., et al. (2012). Patient-specific induced pluripotent stem cells as a model for familial dilated cardiomyopathy. Sci Transl Med 4, 130 ra47.

Tak, T., Sharma, U., Karturi, S., and Gharacholou, S.M. (2018). Familial Tako-tsubo Cardiomyopathy: Clinical and echocardiographic features including magnetic resonance imaging findings. WMJ 117, 171-174.

Takahashi, K., and Yamanaka, S. (2006). Induction of pluripotent stem cells from mouse embryonic and adult fibroblast cultures by defined factors. Cell 126, 663-676. 
Takahashi, K., and Yamanaka, S. (2016). A decade of transcription factor-mediated reprogramming to pluripotency. Nat Rev Mol Cell Biol 17, 183-193.

Takahashi, K., Tanabe, K., Ohnuki, M., Narita, M., Ichisaka, T., Tomoda, K., and Yamanaka, S. (2007). Induction of Pluripotent Stem Cells from Adult Human Fibroblasts by Defined Factors. Cell 131, 861-872.

Takashima, S., Gold, D., and Hartenstein, V. (2013). Stem cells and lineages of the intestine: a developmental and evolutionary perspective. Dev Genes Evol 223, 85-102.

Templin, C., Zweigerdt, R., Schwanke, K., Olmer, R., Ghadri, J.-R., Emmert, M.Y., Müller, E., Küest, S.M., Cohrs, S., Schibli, R., et al. (2012). Transplantation and tracking of human-induced pluripotent stem cells in a pig model of myocardial infarction: assessment of cell survival, engraftment, and distribution by hybrid single photon emission computed tomography/computed tomography of sodium iodide symporter transgene expression. Circulation 126, 430-439.

Templin, C., Ghadri, J.R., Diekmann, J., Napp, L.C., Bataiosu, D.R., Jaguszewski, M., Cammann, V.L., Sarcon, A., Geyer, V., Neumann, C.A., et al. (2015). Clinical features and outcomes of Takotsubo (Stress) Cardiomyopathy. N Engl J Med 373, 929-938.

Thomson, J.A., Itskovitz-Eldor, J., Shapiro, S.S., Waknitz, M.A., Swiergiel, J.J., Marshall, V.S., and Jones, J.M. (1998). Embryonic stem cell lines derived from human blastocysts. Science 282, 1145-1147.

Tiburcy, M., Hudson, J.E., Balfanz, P., Schlick, S., Meyer, T., Liao, M.-L.C., Levent, E., Raad, F., Zeidler, S., Wingender, E., et al. (2017). Defined engineered human myocardium with advanced maturation for applications in heart failure modelling and repair. Circulation 135, 1832-1847.

Tornvall, P., Collste, O., Ehrenborg, E., and Järnbert-Petterson, H. (2016). A case-control study of risk markers and mortality in Takotsubo Stress Cardiomyopathy. J Am Coll Cardiol 67, 19311936.

Trappanese, D.M., Liu, Y., McCormick, R.C., Cannavo, A., Nanayakkara, G., Baskharoun, M.M., Jarrett, H., Woitek, F.J., Tillson, D.M., Dillon, A.R., et al. (2015). Chronic $\beta_{1}$-adrenergic blockade enhances myocardial $\beta_{3}$-adrenergic coupling with nitric oxide-cGMP signaling in a canine model of chronic volume overload: new insight into mechanisms of cardiac benefit with selective $\beta_{1}$ blocker therapy. Basic Res Cardiol 110, 456.

Uchida, Y., Egami, H., Uchida, Y., Sakurai, T., Kanai, M., Shirai, S., Nakagawa, O., and Oshima, T. (2010). Possible participation of endothelial cell apoptosis of coronary microvessels in the genesis of Takotsubo cardiomyopathy. Clin Cardiol 33, 371-377.

Ueyama, T., Kasamatsu, K., Hano, T., Yamamoto, K., Tsuruo, Y., and Nishio, I. (2002). Emotional stress induces transient left ventricular hypocontraction in the rat via activation of cardiac adrenoceptors: a possible animal model of "tako-tsubo" cardiomyopathy. Circ J 66, 712713.

Ueyama, T., Hano, T., Kasamatsu, K., Yamamoto, K., Tsuruo, Y., and Nishio, I. (2003). Estrogen attenuates the emotional stress-induced cardiac responses in the animal model of Tako-tsubo (Ampulla) cardiomyopathy. J Cardiovasc Pharmacol 42 Suppl 1, S117-119. 
Urbach, A., and Benvenisty, N. (2009). Studying early lethality of 45,XO (Turner's syndrome) embryos using human embryonic stem cells. PLoS One 4, e4175.

Veerman, C.C., Mengarelli, I., Guan, K., Stauske, M., Barc, J., Tan, H.L., Wilde, A.A.M., Verkerk, A.O., and Bezzina, C.R. (2016). hiPSC-derived cardiomyocytes from Brugada Syndrome patients without identified mutations do not exhibit clear cellular electrophysiological abnormalities. Sci Rep 6, 30967.

Vitale, C., Rosano, G.M., and Kaski, J.C. (2016). Role of coronary microvascular dysfunction in Takotsubo Cardiomyopathy. Circ J 80, 299-305.

Vreeker, A., van Stuijvenberg, L., Hund, T.J., Mohler, P.J., Nikkels, P.G.J., and van Veen, T.A.B. (2014). Assembly of the cardiac intercalated disk during pre- and postnatal development of the human heart. PLoS One 9, e94722.

Vriz, O., Minisini, R., Citro, R., Guerra, V., Zito, C., De Luca, G., Pavan, D., Pirisi, M., Limongelli, G., and Bossone, E. (2011). Analysis of beta1 and beta2-adrenergic receptors polymorphism in patients with apical ballooning cardiomyopathy. Acta Cardiol 66, 787-790.

van der Wall, E.E., and van Gilst, W.H. (2013). Neurocardiology: close interaction between heart and brain. Neth Heart J 21, 51-52.

Wang, P.-Y., Yu, J., Lin, J.-H., and Tsai, W.-B. (2011). Modulation of alignment, elongation and contraction of cardiomyocytes through a combination of nanotopography and rigidity of substrates. Acta Biomater 7, 3285-3293.

Wang, Y.-K., Zhu, W.-W., Wu, M.-H., Wu, Y.-H., Liu, Z.-X., Liang, L.-M., Sheng, C., Hao, J., Wang, L., Li, W., et al. (2018). Human clinical-grade parthenogenetic ESC-derived dopaminergic neurons recover locomotive defects of nonhuman primate models of Parkinson's disease. Stem Cell Reports 11, 171-182.

Wang, Z.-B., Zhang, X., and Li, X.-J. (2013). Recapitulation of spinal motor neuron-specific disease phenotypes in a human cell model of spinal muscular atrophy. Cell Res 23, 378-393.

Wei, Z., Gao, F., Kim, S., Yang, H., Lyu, J., An, W., Wang, K., and Lu, W. (2013). Klf4 organizes long-range chromosomal interactions with the oct4 locus in reprogramming and pluripotency.

Cell Stem Cell 13, 36-47.

Wells, S.P., Waddell, H.M., Sim, C.B., Lim, S.Y., Bernasochi, G.B., Pavlovic, D., Kirchhof, P., Porrello, E.R., Delbridge, L.M.D., and Bell, J.R. (2019). Cardiomyocyte functional screening: interrogating comparative electrophysiology of high-throughput model cell systems. Am J Physiol Cell Physiol 317, C1256-C1267.

Weng, Z., Kong, C.-W., Ren, L., Karakikes, I., Geng, L., He, J., Chow, M.Z.Y., Mok, C.F., Chan, H.Y.S., Webb, S.E., et al. (2014). A simple, cost-effective but highly efficient system for deriving ventricular cardiomyocytes from human pluripotent stem cells. Stem Cells Dev 23, 1704-1716.

Wernig, M., Lengner, C.J., Hanna, J., Lodato, M.A., Steine, E., Foreman, R., Staerk, J., Markoulaki, S., and Jaenisch, R. (2008). A drug-inducible transgenic system for direct reprogramming of multiple somatic cell types. Nat Biotechnol 26, 916-924. 
Wittstein, I.S., Thiemann, D.R., Lima, J.A.C., Baughman, K.L., Schulman, S.P., Gerstenblith, G., Wu, K.C., Rade, J.J., Bivalacqua, T.J., and Champion, H.C. (2005). Neurohumoral features of myocardial stunning due to sudden emotional stress. N Engl J Med 352, 539-548.

Wright, P.T., Bhogal, N.K., Diakonov, I., Pannell, L.M.K., Perera, R.K., Bork, N.I., Schobesberger, S., Lucarelli, C., Faggian, G., Alvarez-Laviada, A., et al. (2018). Cardiomyocyte membrane structure and cAMP compartmentation produce anatomical variation in $\beta_{2} A R-c A M P$ responsiveness in murine hearts. Cell Rep 23, 459-469.

Wu, Q., and Kling, J.M. (2016). Depression and the risk of myocardial infarction and coronary death: A meta-analysis of prospective cohort studies. Medicine (Baltimore) 95, e2815.

Wu, H., Lee, J., Vincent, L.G., Wang, Q., Gu, M., Lan, F., Churko, J.M., Sallam, K.I., Matsa, E., Sharma, A., et al. (2015). Epigenetic regulation of phosphodiesterases $2 \mathrm{~A}$ and $3 \mathrm{~A}$ underlies compromised $\beta$-Adrenergic signaling in an iPSC model of dilated cardiomyopathy. Cell Stem Cell 17, 89-100.

Yabe, S.G., Fukuda, S., Nishida, J., Takeda, F., Nashiro, K., and Okochi, H. (2019). Induction of functional islet-like cells from human iPS cells by suspension culture. Regen Ther 10,69-76.

Yan, G., Zhu, N., Huang, S., Yi, B., Shang, X., Chen, M., Wang, N., Zhang, G., Talarico, J.A., Tilley, D.G., et al. (2015). Orphan Nuclear Receptor Nur77 inhibits cardiac hypertrophic response to beta-adrenergic stimulation. Molecular and Cellular Biology 35, 3312-3323.

Yang, T., Rubart, M., Soonpaa, M.H., Didié, M., Christalla, P., Zimmermann, W.-H., and Field, L.J. (2015). Cardiac engraftment of genetically-selected parthenogenetic stem cell-derived cardiomyocytes. PLoS ONE 10, e0131511.

Yang, X., Rodriguez, M., Pabon, L., Fischer, K.A., Reinecke, H., Regnier, M., Sniadecki, N.J., Ruohola-Baker, H., and Murry, C.E. (2014). Tri-iodo-I-thyronine promotes the maturation of human cardiomyocytes-derived from induced pluripotent stem cells. J Mol Cell Cardiol 72, 296304.

Yingjun, X., Yuhuan, X., Yuchang, C., Dongzhi, L., Ding, W., Bing, S., Yi, Y., Dian, L., Yanting, X., Zeyu, X., et al. (2019). CRISPR/Cas9 gene correction of HbH-CS thalassemia-induced pluripotent stem cells. Ann Hematol 98, 2661-2671.

You, X., Guo, Z.-F., Cheng, F., Yi, B., Yang, F., Liu, X., Zhu, N., Zhao, X., Yan, G., Ma, X.-L., et al. (2018). Transcriptional up-regulation of relaxin-3 by Nur77 attenuates $\beta$-adrenergic agonistinduced apoptosis in cardiomyocytes. J Biol Chemistry 293, 14001-14011.

Young, C.S., Hicks, M.R., Ermolova, N.V., Nakano, H., Jan, M., Younesi, S., Karumbayaram, S., Kumagai-Cresse, C., Wang, D., Zack, J.A., et al. (2016). A single CRISPR-Cas9 deletion strategy that targets the majority of DMD patients restores dystrophin function in hiPSC-derived muscle cells. Cell Stem Cell 18, 533-540.

Yu, J., Vodyanik, M.A., Smuga-Otto, K., Antosiewicz-Bourget, J., Frane, J.L., Tian, S., Nie, J., Jonsdottir, G.A., Ruotti, V., Stewart, R., et al. (2007). Induced pluripotent stem cell lines derived from human somatic cells. Science 318, 1917-1920.

Yumlu, S., Bashir, S., Stumm, J., and Kühn, R. (2019). Efficient gene editing of human induced pluripotent stem cells using CRISPR/Cas9. Methods Mol Biol 1961, 137-151. 
Yusa, K., Rashid, S.T., Strick-Marchand, H., Varela, I., Liu, P.-Q., Paschon, D.E., Miranda, E., Ordóñez, A., Hannan, N.R.F., Rouhani, F.J., et al. (2011). Targeted gene correction of $\alpha_{1^{-}}$ antitrypsin deficiency in induced pluripotent stem cells. Nature 478, 391-394.

Zafeiriou, M.-P., Bao, G., Hudson, J., Halder, R., Blenkle, A., Schreiber, M.-K., Fischer, A., Schild, D., and Zimmermann, W.-H. (2020). Developmental GABA polarity switch and neuronal plasticity in Bioengineered Neuronal Organoids. Nat Commun 11, 3791.

Zaroff, J.G., Pawlikowska, L., Miss, J.C., Yarlagadda, S., Ha, C., Achrol, A., Kwok, P.-Y., McCulloch, C.E., Lawton, M.T., Ko, N., et al. (2006). Adrenoceptor polymorphisms and the risk of cardiac injury and dysfunction after subarachnoid hemorrhage. Stroke 37, 1680-1685.

Zhang, Z., and Wu, W.-S. (2013). Sodium butyrate promotes generation of human induced pluripotent stem cells through induction of the miR302/367 cluster. Stem Cells Dev. 22, 22682277.

Zhang, J., Wilson, G.F., Soerens, A.G., Koonce, C.H., Yu, J., Palecek, S.P., Thomson, J.A., and Kamp, T.J. (2009). Functional cardiomyocytes derived from human induced pluripotent stem cells. Circ Res 104, e30-41.

Zhang, Q., Jiang, J., Han, P., Yuan, Q., Zhang, J., Zhang, X., Xu, Y., Cao, H., Meng, Q., Chen, L., et al. (2011a). Direct differentiation of atrial and ventricular myocytes from human embryonic stem cells by alternating retinoid signals. Cell Res $21,579-587$.

Zhang, X.-H., Haviland, S., Wei, H., Sarić, T., Fatima, A., Hescheler, J., Cleemann, L., and Morad, M. (2013). $\mathrm{Ca}^{2+}$ signaling in human induced pluripotent stem cell-derived cardiomyocytes (iPS-CM) from normal and catecholaminergic polymorphic ventricular tachycardia (CPVT)afflicted subjects. Cell Calcium 54, 57-70.

Zhang, Z., Gao, Y., Gordon, A., Wang, Z.Z., Qian, Z., and Wu, W.-S. (2011b). Efficient generation of fully reprogrammed human iPS cells via polycistronic retroviral vector and a new cocktail of chemical compounds. PLoS ONE 6, e26592.

Zhang, Z., Jin, S., Teng, X., Duan, X., Chen, Y., and Wu, Y. (2017). Hydrogen sulfide attenuates cardiac injury in takotsubo cardiomyopathy by alleviating oxidative stress. Nitric Oxide 67, 1025.

Zhao, Z., Fan, L., and Frick, K.M. (2010). Epigenetic alterations regulate estradiol-induced enhancement of memory consolidation. Proc Natl Acad Sci U S A 107, 5605-5610.

Zhou, W., and Freed, C.R. (2009). Adenoviral gene delivery can reprogram human fibroblasts to induced pluripotent stem cells. Stem Cells 27, 2667-2674.

Zhou, H., Wu, S., Joo, J.Y., Zhu, S., Han, D.W., Lin, T., Trauger, S., Bien, G., Yao, S., Zhu, Y., et al. (2009). Generation of induced pluripotent stem cells using recombinant proteins. Cell Stem Cell 4, 381-384.

Zhu, H., Scharnhorst, K.S., Stieg, A.Z., Gimzewski, J.K., Minami, I., Nakatsuji, N., Nakano, H., and Nakano, A. (2017). Two dimensional electrophysiological characterization of human pluripotent stem cell-derived cardiomyocyte system. Sci Rep 7, 43210. 
Zhu, W.-Z., Xie, Y., Moyes, K.W., Gold, J.D., Askari, B., and Laflamme, M.A. (2010). Neuregulin/ErbB signaling regulates cardiac subtype specification in differentiating human embryonic stem cells. Circ Res 107, 776-786.

Zieleń, P., Klisiewicz, A., Januszewicz, A., Prejbisz, A., Kabat, M., Peczkowska, M., Stepińska, J., and Hoffman, P. (2010). Pheochromocytoma-related "classic" takotsubo cardiomyopathy. J Hum Hypertens 24, 363-366.

Zubrinich, C.M., Farouque, H.M.O., Rochford, S.E., and Sutherland, M.F. (2008). Tako-tsubolike cardiomyopathy after EpiPen administration. Intern Med J 38, 862-865. 


\section{Acknowledgments}

During my $\mathrm{PhD}$ student life, I met a lot of incredibly friendly and helpful people, which I would like to thank in this part.

Firstly, PD Dr. rer. Nat. Katrin Streckfuß-Bömeke for providing the opportunity to start my $\mathrm{PhD}$ in her lab and for her unconditional support also during hard times. For her time discussing experiments and developing new approaches together.

I want to thank Prof. Dr. mult. Thomas Meyer and Prof. Dr. Michael Zeisberg for fruitful discussions during my thesis committee meetings. Special Thanks to Thomas for being the second reviewer and providing additional input and ideas for new experiments.

I want to express my gratitude to Prof. Dr. Ralf Dressel, Prof. Dr. Rüdiger Behr, and PD Dr. Laura Zelarayán-Behrend for being part of my examination committee despite their busy schedules.

I want to thank Sandra Georgie for excellent technical support, Dr. Luis Haupt and Dr. Sabine Rebs for fruitful discussions and the whole staff of the Streckfuß-Bömeke lab for help, discussions, and a lot of fun.

I express my gratitude for everybody helping and teaching me: Dr. Lukas Cyganek for introduction to the calcium measurements. Dr. Malte Tiburcy and Dr. Norman Liaw for their help with the EHM. Dr. Isabel Schellinger for fruitful discussion and help with the Oil Red $\mathrm{O}$ analysis. Dr. Daniela Hübscher for HIV screening.

Thank you, Dr. Michael Stauske and Simin Chen, for emotional support, fruitful discussions, and proofreading by Simin. Dr. Armin Feist for proofreading and fruitful discussion in non-work-related topics.

I want to thank my parents, my sister and my friends for always having my back and supporting me through this journey. A special thanks goes to Jana Kuchta for being the light of my life and supporting me through these challenging times. 


\section{Curriculum Vitae}

\section{3-2020 PhD Molecular Medicine}

Georg-August-University, Göttingen

Thesis: Modeling Takotsubo syndrome with patient-specific induced pluripotent stem cell-derived cardiomyocytes

\section{1-2013 Master of Molecular Medicine}

Georg-August-University, Göttingen

final degree: 1.18

Master thesis: modeling catecholaminergic

polymorphic ventricular tachycardia with

induced pluripotent stem cells.

\section{8-2011 Bachelor of Molecular Medicine}

Georg-August-University, Göttingen

Final degree: 1.38

Bachelor thesis: The non-canonical Wnt-Pathway during the differentiation of human embryonic stem cells to cardiomyocytes. 


\section{Publications and Presentations}

\section{Publications}

Borchert, T., Hübscher, D., Guessoum, C.I., Lam, T.-D.D., Ghadri, J.R., Schellinger, I.N., Tiburcy, M., Liaw, N.Y., Li, Y., Haas, J., et al. (2017). Catecholamine-Dependent $\beta$ Adrenergic Signaling in a Pluripotent Stem Cell Model of Takotsubo Cardiomyopathy. $J$ Am Coll Cardiol 70, 975-991.

Hübscher, D., Rebs, S., Haupt, L., Borchert, T., Guessoum, C.I., Treu, F., Köhne, S., Maus, A., Hambrecht, M., Sossalla, S., et al. (2019). A High-Throughput Method as a Diagnostic Tool for HIV Detection in Patient-Specific Induced Pluripotent Stem Cells Generated by Different Reprogramming Methods. Stem Cells Int 2019, 2181437.

Stauske, M., Rodriguez Polo, I., Haas, W., Knorr, D.Y., Borchert, T., Streckfuss-

Bömeke, K., Dressel, R., Bartels, I., Tiburcy, M., Zimmermann, W.-H., et al. (2020). NonHuman Primate iPSC Generation, Cultivation, and Cardiac Differentiation under Chemically Defined Conditions. Cells 9, 1349.

\section{Presentations}

\section{Oral Presentations}

T. Borchert, C. I. Guessoum, C. Templin, G. Hasenfuß, K. Streckfuß-Bömeke (Göttingen; Zürich, $\mathrm{CH}$ ). „Role of BAG3 in Takotsubo cardiomyopathy using human inducedpluripotent stem cell-derived cardiomyocytes" Borchert et al., oral presentation at DGK $82^{\text {nd }}$ annual meeting, Mannheim

Modeling Takotsubo syndrome with induced pluripotent stem cells. Borchert et al., oral presentation at Molmed Retreat 2017, Wernigerode

Patient specific iPSC-CMs for studying the disease mechanism of TTS. Borchert et al., oral presentation at DZHK retreat 2016, Bad Aibling 


\section{Poster presentations}

Modeling Takotsubo Cardiomyopathy using human induced pluripotent stem cell derived cardiomyocytes. Borchert et al., poster presentation at 8th international meeting of the stem cell network north Rhine Westphalia 2015, Bonn

Patient-specific induced pluripotent stem cell-derived cardiomyocytes for studying disease mechanisms of the takotsubo cardiomyopathy: proof of principle. Borchert et al., poster presentation at DGK $82^{\text {nd }}$ annual meeting, Mannheim

A patient-specific induced pluripotent stem cell model of Takotsubo Syndrome uncovers differences in electrical activity and functionality of calcium homeostasis between TTS and control cardiomyocytes. Borchert et al., poster presentation at DGK Herztage 2017, Berlin 


\title{
7. Affidavit
}

\author{
I hereby declare that the submitted doctoral thesis entitled: \\ "Modeling Takotsubo syndrome with patient-specific induced pluripotent stem cell- \\ derived cardiomyocytes" \\ has been written independently with no other aids or sources than quoted.
}

Thomas Borchert

Magdeburg, November 2020 